\title{
INTERSECTIONS OF ALGEBRAIC AND ALGEBROID VARIETIES
}

\author{
BY \\ CLAUDE CHEVALLEY
}

Introduction. The object of this paper is to provide a local theory for the intersection multiplicities of algebraic varieties.

The notion of intersection multiplicity of two algebraic varieties has been for the first time put on a solid base by van der Waerden. We consider the present step as an improvement on the van der Waerden theory for the following reasons:

(1) Esthetically, it seems natural to connect the multiplicity of a component $M$ of the intersection of two varieties $U$ and $V$ with the local properties of $U$ and $V$ in the neighbourhood of $M$.

(2) Our theory includes an intersection theory for algebroid varieties.

(3) The theory of van der Waerden fails to attribute a multiplicity to a component $M$ of the intersection of $U$ and $V$ in the case where, although $M$ has the suitable dimension, some other components have too high dimensions.

The starting point of our considerations has been the observation that the multiplicity of the origin $O$ in the intersection of two curves $f(X, Y)=0$, $g(X, Y)=0$ may be defined to be the degree of the field extension $K((X, Y)) / K((f, g))$, where $K((X, Y))$ is the field of quotients of the ring of power series in $X, Y$ with coefficients in the basic field $K$, and where $K((f, g))$ is the field of quotients of the ring of those power series in $X, Y$ which can be expressed as power series in $f$ and $g$. From there, I was led to the definition of multiplicity of a local ring with respect to a system of parameters, and then to the general notion of intersection multiplicity. In order to achieve this generalization, I have made extensive use of the notion of local ring, introduced by Krull.

This paper is divided into three parts. Part I contains some algebraic preparations. It is concerned with the study of the properties of a certain class of local rings, which I have called geometric local rings. The most important results in this first part are:

(1) what I have called the theorem of transition ( $\$ 4$, p. 22$)$, because it is the tool by the use of which we can reduce questions of intersection multiplicities for algebraic varieties to similar questions for algebroid varieties;

(2) the associativity formula for multiplicities in local rings which is the source not only of the associativity formula for intersections, but of most other properties of intersection multiplicities as well.

Presented to the Society, August 14, 1944; received by the editors February 12, 1944. 
Parts II and III are concerned with the intersection theory of algebroid and algebraic varieties respectively. Each of these parts begins with a short reminder of the main definitions. These are not meant to provide a first introduction to the notions with which algebraic geometry deals; their object is rather to determine unequivocally which one of the various possible points of view we adopt. In order to be able to reduce the theory for algebraic varieties to the corresponding theory for algebroid varieties, we show that an algebraic variety $U$ splits up in the neighbourhood of one of its points into a certain number of algebroid varieties, which we call the sheets of $U$ at the point.

Another intersection theory of algebraic varieties will be published shortly by A. Weil. I have been in constant communication with A. Weil during the writing of this paper; many of the ideas involved can be traced back to discussions of the subject between him and myself. It is therefore impossible for me to acknowledge with precision the extent of my indebtedness to him. Nevertheless, it can be said definitely that the statement of the "projection formula" and the knowledge of the fact that all properties of intersections can be derived from three basic theorems (namely, the theorem on intersection of product varieties, the projection formula and the formula of associativity) are both due specifically to A. Weil.

Terminology, references, and so on. Numerous references will be made to my paper On the theory of local rings, Ann. of Math. vol. 44 (1943) p. 690. This paper will be denoted by the abbreviation L.R.

We adopt the conventions of terminology which were explained in the beginning of L.R., and we introduce some new ones, which we now proceed to explain.

In parts II and III, the letter $K$ will consistently represent a fixed algebraically closed field. We shall of ten make use of certain symbols (such as $\left.X_{1}, \cdots, X_{n}, \cdots, Y_{j}, \cdots\right)$ which we call letters. These letters are meant to represent arguments of polynomials or power series. It follows that letters which are represented by different symbols are automatically assumed to be different and to be analytically independent over $K$. If $X_{1}, \cdots, X_{n}$ are letters, $K\left[\left[X_{1}, \cdots, X_{n}\right]\right]$ represents the ring of power series in $X_{1}, \cdots, X_{n}$ with coefficients in $K ; K\left(\left(X_{1}, \cdots, X_{n}\right)\right)$ represents the field of quotients of $K\left[\left[X_{1}, \cdots, X_{n}\right]\right]$.

More generally, if $\mathfrak{o}$ is a complete semi-local ring and contains a field $K_{0}$, and if $x_{1}, \ldots, x_{n}$ are in the intersection of the maximal prime ideals of 0 , then $K_{0}\left[\left[x_{1}, \cdots, x_{n}\right]\right]$ represents the set of elements of 0 which can be expressed as power series in $x_{1}, \cdots, x_{n}$ with coefficients in $K_{0}$. If this ring has no zero divisor not equal to 0 , its field of quotients is denoted by $K_{0}\left(\left(x_{1}, \cdots, x_{n}\right)\right)$.

If $\mathfrak{p}$ is a prime ideal in a Noetherian ring $\mathfrak{o}$, we denote by $o_{\mathfrak{p}}$ the ring of quotients of $\mathfrak{p}$ with respect to $D$ in the sense which was defined in my paper 
On the notion of the ring of quotients of a prime ideal, Bull. Amer. Math. Soc. vol. 50 (1944) p. 93. If $\mathfrak{w}$ is the intersection of all primary ideals of $o$ which are contained in $\mathfrak{p}, \mathfrak{D}_{\mathfrak{p}}$ contains $\mathfrak{o} / \mathfrak{w}$ as a subring and is the ring of quotients (in the ordinary sense) of $\mathfrak{p} / \mathfrak{w}$ with respect to $0 / \mathfrak{w}$. The homomorphism which assigns to every element of $\mathfrak{D}$ its residue class modulo $\mathfrak{w}$ is called the natural homomorphism of $\mathrm{o}$ into $\mathrm{D}_{\mathfrak{p}}$.

Throughout this paper we reserve the name of "varieties" to the irreducible varieties over an algebraically closed field.

The cross references in the present paper are made according to the following principle: if no indication of section is given, the reference refers to some statement or formula contained in the same section; a similar convention holds if no indication of part is given. The propositions, lemmas, formulas and definitions are numbered starting with 1 in each section; the theorems are numbered starting with 1 in each part.

\section{PART I}

1. Geometric local rings. The local rings which occur in algebraic geometry are of a somewhat special type and have a certain number of properties of their own which we propose now to investigate.

We shall say that a ring $\mathfrak{r}$ is of type $\mathfrak{r}(n ; K)$ if the following conditions are satisfied: $K$ is a field with infinitely many elements which is contained in $\mathfrak{r}$; if the characteristic $p$ of $K$ is not equal to 0 , then $\left[K: K^{p}\right]$ is finite; $\mathfrak{r}$ contains $n$ elements $x_{1}, \cdots, x_{n}$ which are algebraically independent over $K$; $\mathfrak{r}$ is the ring of quotients with respect to $K\left[x_{1}, \cdots, x_{n}\right]$ of the prime ideal generated in this ring by $x_{1}, \cdots, x_{n}$. These conditions imply that $\mathfrak{r}$ is a regular local ring of dimension $n$ and that $x_{1}, \cdots, x_{n}$ form a regular system of parameters in $\mathfrak{r}$ (cf. Definition 3, L.R., \$III, p. 704). We shall say that $\left\{x_{1}, \cdots, x_{n}\right\}$ is a special system of parameters in $r$. Every special system of parameters is regular, but not conversely.

Now, we introduce the smallest class $\left({ }^{1}\right)$ (s) of rings which satisfies the following conditions:

(1) Every ring of type $\mathfrak{r}(n ; K)$ belongs to $(S)$

(2) If $\mathrm{o} \in\left(\mathrm{S}\right.$ and if $\mathfrak{p}$ is a prime ideal in $\mathrm{o}$, then $\mathrm{o} / \mathrm{p}$ and $\mathrm{o}_{\mathrm{p}}$ belong to $\mathrm{S}$.

(3) If a local ring o belongs to $\mathbb{S}$, then any completion of $\mathrm{o}$ belongs to $\mathbb{B}$.

Our object is to investigate the common properties of all rings of $\mathrm{B}$. In particular, we shall prove that every ring in $(B)$ is a local ring; we shall then be justified in calling geometric local rings the rings which belong to (5).

To begin with, it is clear that the class of Noetherian rings has the properties (1), (2), (3). It follows that every ring in $(5)$ is Noetherian. Moreover,

(1) In order to avoid the difficulties of a logical nature which are involved in the consideration of the class of all rings, we may limit ourselves to the consideration of those rings whose elements belong to some a priori given set of suitably high cardinal number (the power of continuum will be sufficient in all geometric applications). 
a similar argument shows that, if $\boldsymbol{D}$ is any ring in $(\xi)$, the nonunits in $\boldsymbol{D}$ form an ideal. In order to prove that every ring $D$ in $B$ is a local ring, we still have to prove that $\mathrm{D}$ contains a basic field in the sense of Definition 1, L.R., §III, p. 701.

We shall say that a ring $\mathfrak{r}$ is of type $\overline{\mathfrak{r}}(n, m ; K)$ if the following conditions are satisfied: $\mathfrak{r}$ contains as a subring a ring $\mathfrak{r}_{0}$ of type $\mathfrak{r}(n ; K) ; \mathfrak{r}$ contains a completion $\tilde{\mathfrak{r}}_{0}$ of $\mathfrak{r}_{0}$; there exists a special system of parameters $\left\{x_{1}, \cdots, x_{n}\right\}$ in $\mathfrak{r}_{0}$ such that $\mathfrak{r}$ is the ring of quotients with respect to $\mathfrak{f}_{0}$ of the prime ideal generated by $x_{m+1}, \cdots, x_{n}$ in $\overline{\mathfrak{r}}_{0}$. We shall also say that $\left\{x_{1}, \cdots, x_{n}\right\}$ is a special subset of $\mathfrak{r}$ and that $\left\{x_{m+1}, \cdots, x_{n}\right\}$ is a special system of parameters in $\overline{\mathfrak{r}}$. These conditions imply that $x_{1}, \cdots, x_{n}$ are analytically independent over $K$; $\overline{\mathfrak{r}}_{0}$ is the ring $K\left[\left[x_{1}, \cdots, x_{n}\right]\right]$ and $\mathfrak{r}$ contains the field $K\left(\left(x_{1}, \cdots, x_{m}\right)\right)$. The nonunits in $\mathfrak{r}$ form a prime ideal which is the ideal generated by $x_{m+1}, \cdots, x_{n}$, and $K\left(\left(x_{1}, \cdots, x_{m}\right)\right)$ is a complete system of representatives for the residue classes modulo the ideal of nonunits. It follows that $r$ is a regular local ring and that $x_{m+1}, \cdots, x_{n}$ form a regular system of parameters in $\mathfrak{r}$. If $m=0$, $\mathfrak{r}$ coincides with the ring $K\left[\left[x_{1}, \cdots, x_{n}\right]\right]$.

We shall say that a ring $\mathfrak{b}$ admits a nucleus $\mathfrak{r}$ if the following conditions are satisfied: (1) $\mathfrak{r}$ is a ring which is either of the type $\mathfrak{r}(n ; K)$ or $\overline{\mathfrak{r}}(n, m ; K)$; (2) $\mathfrak{r}$ is a subring of $\mathfrak{o}$ and no element in $\mathfrak{r}$ not equal to 0 becomes a zero divisor in $\mathfrak{D}$; (3) there exists a subring $\Im$ of $\mathfrak{b}$ which contains $\mathfrak{r}$, which is finite over $\mathfrak{r}$, and which has the property that $\mathfrak{o}$ is the ring of quotients with respect to $\Im$ of some maximal prime ideal in $\Im\left({ }^{2}\right)$. If these conditions are satisfied, we shall say that $\Im$ is an intermediary ring in $\mathfrak{o}$ with respect to $\mathfrak{r}$. It is clear that a ring $\mathfrak{D}$ which admits a nucleus is a local ring in which any basic field of $\mathfrak{r}$ is a basic field. An intermediary ring $\Im$ is a semi-local ring (by Proposition 3, L.R., §II, p. 694), and the maximal prime ideals in $\Im$ are the prime ideals which contain the ideal of nonunits in $\mathfrak{r}$. We shall prove later that every ring of the class $\&$ admits a nucleus.

Lemma 1. Assume that $x_{1}, \cdots, x_{n}$ are algebraically independent over a field $K$ which contains infinitely many elements. If $y$ is an element of $K\left[x_{1}, \cdots, x_{n}\right]$ which does not belong to $K$, there exists a set $\left\{y_{1}, \cdots, y_{n}\right\}$ of $n$ elements in $K\left[x_{1}, \cdots, x_{n}\right]$ such that $y_{1}=y$ and such that $K\left[x_{1}, \cdots, x_{n}\right]$ is finite over $K\left[y_{1}, \cdots, y_{n}\right]$.

Write $y=P\left(x_{1}, \cdots, x_{n}\right)$, where $P$ is a polynomial of degree $d>0$ with coefficients in $K$, and denote by $F$ the homogeneous component of degree $d$ of $P$. Since $K$ contains infinitely many elements, we can find elements $a_{2}, \cdots, a_{n}$ in $K$ such that $F\left(1, a_{2}, \cdots, a_{n}\right) \neq 0$. Set $y_{1}=y_{1} y_{i}=x_{i}-a_{i} x_{1}$ $(2 \leqq i \leqq n)$. Then, the coefficient of the highest power of $X_{1}$ in the polynomial $P\left(X_{1}, X_{2}+a_{2} X_{1}, \cdots, X_{n}+a_{n} X_{1}\right)$ is not equal to 0 ; since $P\left(x_{1}, y_{2}+a_{2} x_{1}, \cdots\right.$,

(2) This definition implies that this maximal prime ideal must contain all prime divisors of $\Im$, for otherwise $\Im$ would not be a subring of 0 . 
$\left.y_{n}+a_{n} x_{1}\right)-y_{1}=0$, it follows that $x_{1}$ is integral over $K\left[y_{1}, \cdots, y_{n}\right]$ and therefore that $K\left[x_{1}, \cdots, x_{n}\right]$ is finite over $K\left[y_{1}, \cdots, y_{n}\right]$.

Lemma $2\left(^{3}\right)$. The situation being as described in Lemma 1, let $\mathfrak{p}$ be a prime ideal in $K\left[x_{1}, \cdots, x_{n}\right]$. Then there exists $a$ set of $n$ elements $y_{1}, \cdots, y_{n}$ in $K\left[x_{1}, \cdots, x_{n}\right]$ such that: (1) $K\left[x_{1}, \cdots, x_{n}\right]$ is finite over $K\left[y_{1}, \cdots, y_{n}\right] ;(2)$ the ideal $\mathfrak{p} \cap K\left[y_{1}, \cdots, y_{n}\right]$ can be generated in $K\left[y_{1}, \cdots, y_{n}\right]$ by $y_{m+1}, \cdots, y_{n}$, where $m$ is an integer less than $n$.

Let us say that a set $\left\{y_{1}, \cdots, y_{n}\right\}$ composed of $n$ elements of $K\left[x_{1}, \cdots, x_{n}\right]$ is a system of integrity $\left({ }^{4}\right)$ in this ring if $K\left[x_{1}, \cdots, x_{n}\right]$ is finite over $K\left[y_{1}, \cdots, y_{n}\right]$; let us say that a subset $S$ of $\mathfrak{p}$ is a set of integrity for $\mathfrak{p}$ if the following conditions are satisfied: $S$ is contained in some system of integrity of $K\left[x_{1}, \cdots, x_{n}\right]$, but no subset of $\mathfrak{p}$ which strictly $\left(^{5}\right)$ contains $S$ has the same property. Let $\left\{y_{1}, \cdots, y_{n}\right\}$ be a system of integrity which contains a set of integrity $\left\{y_{m+1}, \cdots, y_{n}\right\}$ for $\mathfrak{p}$. Then $\mathfrak{p} \cap K\left[y_{1}, \cdots, y_{m}\right]$ contains only 0 . In fact, assume for a moment that this set contains an element $z \neq 0$. Since $z \in \mathfrak{p}, z$ does not belong to $K$; by Lemma $1, z$ belongs to a system of integrity $\left\{z_{1}, \cdots, z_{m}\right\}$ in $K\left[y_{1}, \cdots, y_{m}\right]$. It is obvious that $K\left[y_{1}, \cdots, y_{n}\right]$ is finite over $K\left[z_{1}, \cdots, z_{m}, y_{m+1}, \cdots, y_{n}\right]$; therefore $\left\{z_{1}, \cdots, z_{m}, y_{m+1}, \cdots, y_{n}\right\}$ is a system of integrity. But this is impossible because this set contains $\left\{z, y_{m+1}, \cdots, y_{n}\right\}$ which is a subset of $\mathfrak{p}$ and strictly contains $\left\{y_{m+1}, \cdots, y_{n}\right\}$. Since $p$ contains $y_{m+1}, \cdots, y$, and has only 0 in common with $K\left[y_{1}, \cdots, y_{m}\right]$, it is clear that $\mathfrak{p} \cap K\left[y_{1}, \cdots, y_{n}\right]$ is generated by $y_{m+1}, \cdots, y_{n}$.

LEMMA $3\left(^{6}\right)$. If $\mathrm{D}$ is a local ring, every nonunit in $\mathrm{o}$ which is not a zero divisor belongs to some system of parameters in $\mathrm{D}$.

Let $y_{1}$ be a nonunit which is not a zero divisor, and let $n$ be the dimension of $\mathrm{o}$. Then $\mathrm{o} / \mathrm{o} y_{1}$ is of dimension $n^{\prime}<n$ (cf. Proposition 6, L.R., §III, p. 702). Let $y_{2}, \cdots, y_{n^{\prime}+1}$ be elements of $\mathrm{o}$ whose residue classes modulo $\mathrm{D} y_{1}$ form a system of parameters in $\mathfrak{o} / \mathfrak{o} y_{1}$. If a prime ideal $p$ contains $y_{1}, \cdots, y_{n^{\prime}+1}$, then $\mathfrak{p}$ contains $\mathfrak{o} y_{1}$, and $\mathfrak{p} / \mathfrak{o} y_{1}$ is the ideal of nonunits in $\mathfrak{o} / \mathfrak{o} y_{1}$. It follows that $\mathfrak{p}$ is the ideal of nonunits in $\mathfrak{D}$. Since $n^{\prime}+1 \leqq n$, we have $n^{\prime}+1=n$ and $y_{1}, \cdots, y_{n^{\prime}+1}$ form a system of parameters in $\mathbf{0}$.

Lemma 4. Let $\mathrm{o}$ be a local ring of the form $K\left[\left[x_{1}, \cdots, x_{n}\right]\right]$ where $x_{1}, \cdots, x_{n}$ are analytically independent over $K$. Let $\mathfrak{p}$ and $\mathfrak{q}$ be prime ideals in $\mathrm{v}$ such that

(3) Lemma 2 is substantially equivalent to a well known lemma due to Fr. Noether. For a general proof of this lemma (including the case of a finite basic field), cf. O. Zariski, Foundations of a general theory of birational correspondences, Trans. Amer. Math. Soc. vol. 53 (1943) pp. 506, 507.

(4) The systems of integrity in $K\left[x_{1}, \cdots, x_{n}\right]$ play a role which has a certain analogy with the role played by systems of parameters in a local ring.

(5) We say that a set $S^{\prime}$ strictly contains $S$ if $S \subset S^{\prime}, S \neq S^{\prime}$.

(6) The proof of Lemma 3 is implicitly contained in the proof of Proposition 7, L.R., §III, p. 703. 
$\mathfrak{p} \subset \mathfrak{q}$. Then there exists a system of parameters $\left\{y_{1}, \cdots, y_{n}\right\}$ in o such that $\mathfrak{p} \cap K\left[\left[y_{1}, \cdots, y_{n}\right]\right]$ is the ideal generated in $K\left[\left[y_{1}, \cdots, y_{n}\right]\right]$ by $y_{r+1}, \cdots, y_{n}$ and that $\mathrm{g} \cap K\left[\left[y_{1}, \ldots, y_{n}\right]\right]$ is the ideal generated in $K\left[\left[y_{1}, \cdots, y_{n}\right]\right]$ by $y_{m+1}, \cdots, y_{n}$ ( $m$ and $r$ are integers such that $\left.m \leqq r\right)$.

Let $\mathfrak{a}$ be an ideal in a local ring $\boldsymbol{0}$. We say that a subset $S$ of $\mathfrak{a}$ is a parametric $\operatorname{set}\left({ }^{7}\right)$ for $\mathfrak{a}$ if $S$ is contained in some system of parameters in 0 but no subset of $a$ which strictly contains $S$ has the same property. Let $\left\{z_{1}, \cdots, z_{n}\right\}$ be a system of parameters in $K\left[\left[x_{1}, \cdots, x_{n}\right]\right]$ which contains a parametric set $\left\{z_{r+1}, \cdots, z_{n}\right\}$ of $\mathfrak{p}$. Then $\mathfrak{p}$ has only 0 in common with the ring $K\left[\left[z_{1}, \cdots, z_{r}\right]\right]$. In fact, assume for a moment that $\mathfrak{p} \cap K\left[\left[z_{1}, \cdots, z_{r}\right]\right]$ contains an element $u \neq 0$. Then $u$ is not a unit; by Lemma $3, u$ belongs to a system of parameters $\left\{u_{1}, \cdots, u_{r}\right\}$ in $K\left[\left[z_{1}, \cdots, z_{r}\right]\right]$. It is clear that $K\left[\left[z_{1}, \ldots, z_{n}\right]\right]$ is finite over $K\left[\left[u_{1}, \ldots, u_{r}, z_{r+1}, \ldots, z_{n}\right]\right]$; therefore the set $\left\{u_{1}, \cdots, u_{r}, z_{r+1}, \cdots, z_{n}\right\}$ is a system of parameters in $K\left[\left[x_{1}, \cdots, x_{n}\right]\right]$. But this is impossible, since this set contains $\left\{u, z_{r+1}, \cdots, z_{n}\right\}$, which is a subset of $\mathfrak{p}$ and which strictly contains $\left\{z_{r+1}, \cdots, z_{n}\right\}$. Since $\mathfrak{p} \cap K\left[\left[z_{1}, \cdots, z_{r}\right]\right]=\{0\}$, it is clear that $\mathfrak{p} \cap K\left[\left[z_{1}, \cdots, z_{n}\right]\right]$ is the ideal generated by $z_{r+1}, \cdots, z_{n}$ in $K\left[\left[z_{1}, \cdots, z_{n}\right]\right]$.

Proceeding in the same way, we can find a system of parameters $\left\{y_{1}, \cdots, y_{r}\right\}$ in $K\left[\left[z_{1}, \cdots, z_{r}\right]\right]$ such that $q \cap K\left[\left[y_{1}, \cdots, y_{r}\right]\right]$ is the ideal generated by $y_{m+1}, \cdots, y_{r}$ in $K\left[\left[y_{1}, \cdots, y_{r}\right]\right]$ (where $m$ is an integer). Set $y_{i}=z_{i}$ for $i>r$; we see easily that the set $\left\{y_{1}, \cdots, y_{n}\right\}$ has the required properties.

LeMma 5. Let $\mathfrak{o}$ be a ring which admits a nucleus $\mathfrak{r}$ and let $\mathfrak{p}$ be a prime ideal in $\mathfrak{o}$. Then $\mathrm{o}$ admits a nucleus $\mathfrak{r}^{\prime} \subset \mathfrak{r}$ with the property that $\mathfrak{p} \cap \mathfrak{r}^{\prime}$ is the ideal generated in $\mathfrak{r}^{\prime}$ by a subset of some special system of parameters in $\mathfrak{r}^{\prime}$.

Assume first that $\mathfrak{r}$ is of type $\mathfrak{r}(n ; K)$, and let $\left\{x_{1}, \cdots, x_{n}\right\}$ be a special system of parameters in $\mathfrak{r}$. By Lemma 2 , we can find a subset $\left\{y_{1}, \cdots, y_{n}\right\}$ of $\mathfrak{r}$ such that (a) $K\left[x_{1}, \cdots, x_{n}\right]$ is finite over $K\left[y_{1}, \ldots, y_{n}\right]$ and (b) $\mathfrak{p} \cap K\left[y_{1}, \cdots, y_{n}\right]$ is the ideal generated by $y_{m+1}, \cdots, y_{n}$ in $K\left[y_{1}, \cdots, y_{n}\right]$. The elements $y_{m+1}, \cdots, y_{n}$ must belong to the ideal generated by $x_{1}, \cdots, x_{n}$ in $K\left[x_{1}, \cdots, x_{n}\right]$. If $i \leqq m$, there exists an element $a_{i} \in K$ such that $y_{i}-a_{i}=y_{i}^{\prime}$ belongs to the ideal generated by $x_{1}, \cdots, x_{n}$ in $K\left[x_{1}, \cdots, x_{n}\right]$. In this case, we take $\mathfrak{r}^{\prime}$ to be the ring of quotients with respect to $K\left[y_{1}^{\prime}, \cdots, y_{m}^{\prime}\right.$, $\left.y_{m+1}, \cdots, y_{n}\right]$ of the ideal generated in this ring by $y_{1}^{\prime}, \cdots, y_{m}^{\prime}, y_{m+1}, \cdots, y_{n}$. It is clear that $\mathfrak{p} \cap \mathfrak{r}^{\prime}$ is the ideal generated by $y_{m+1}, \cdots, y_{n}$ in $\mathfrak{r}^{\prime}$. Moreover, the ring generated by $K\left[x_{1}, \cdots, x_{n}\right]$ and $\mathfrak{r}^{\prime}$ is finite over $\mathfrak{r}^{\prime}$.

Assume now that $\mathfrak{r}$ is of the type $\overline{\mathfrak{r}}(n, m ; K)$, and let $\left\{x_{1}, \cdots, x_{n}\right\}$ be a special subset of $\mathfrak{r}$. If $\mathfrak{q}$ is the maximal prime ideal of $\mathfrak{b}$, it follows from Lemma 4 that there exists a system of parameters $\left\{y_{1}, \cdots, y_{n}\right\}$ in $K\left[\left[x_{1}, \cdots, x_{n}\right]\right]$ with the following properties: $\mathfrak{p} \cap K\left[\left[y_{1}, \cdots, y_{n}\right]\right]$ is the ideal generated

(7) Observe the analogy with the proof of Lemma 2 above. 
by $y_{r+1}, \cdots, y_{n}$ in $K\left[\left[y_{1}, \cdots, y_{n}\right]\right] ; \mathfrak{q} \cap K\left[\left[y_{1}, \cdots, y_{n}\right]\right]$ is the ideal generated by $y_{m^{\prime}+1}, \cdots, y_{n}$ in $K\left[\left[y_{1}, \cdots, y_{n}\right]\right]$, where $m^{\prime}$ and $r$ are integers such that $m^{\prime} \leqq r$. The ideal $\mathfrak{q} \cap K\left[\left[x_{1}, \ldots, x_{n}\right]\right]=(\mathfrak{q} \cap \mathfrak{r}) \cap K\left[\left[x_{1}, \ldots, x_{n}\right]\right]$ is the ideal generated by $x_{m+1}, \cdots, x_{n}$ in $K\left[\left[x_{1}, \cdots, x_{n}\right]\right]$ and is therefore of dimension $m$. Since $K\left[\left[x_{1}, \cdots, x_{n}\right]\right] /\left(\mathfrak{q} \cap K\left[\left[x_{1}, \cdots, x_{n}\right]\right]\right)$ is finite over $K\left[\left[y_{1}, \cdots, y_{n}\right]\right] /\left(q \cap K\left[\left[y_{1}, \cdots, y_{n}\right]\right]\right)$, it follows that $m^{\prime}=m$. In this case, we denote by $\mathfrak{r}^{\prime}$ the ring of quotients with respect to $K\left[\left[y_{1}, \cdots, y_{n}\right]\right]$ of the ideal generated in this ring by $y_{m+1}, \cdots, y_{n}$. It is clear that $\mathfrak{r}^{\prime}$ is of type $\tilde{\mathfrak{r}}(n, m ; K)$, that $\mathfrak{r}^{\prime} \subset \mathfrak{o}$, and that $\mathfrak{p} \cap \mathfrak{r}^{\prime}$ is the ideal generated in $\mathfrak{r}^{\prime}$ by $y_{r+1}, \cdots, y_{n}$. Moreover, the ring generated by $\mathfrak{r}^{\prime}$ and $K\left[\left[x_{1}, \cdots, x_{n}\right]\right]$ is finite over $\mathfrak{r}^{\prime}$.

Let $\Im$ be an intermediary ring of $\mathfrak{o}$ with respect to $\mathfrak{r}$. Then we have $\Im=\sum_{i=1}^{h} \mathrm{r} c_{i}, c_{i} c_{j}=\sum_{k=1}^{h} a_{i j k} c_{k}, a_{i j k} \in \mathfrak{r}$. In the first case (that is, when $\mathfrak{r}$ is of the type $\mathfrak{r}(n, K))$ we can find an element $d$ in $K\left[x_{1}, \cdots, x_{n}\right]$ which is such that $d$ is a unit in $\mathfrak{r}$ and that $d a_{i j k} \in K\left[x_{1}, \cdots, x_{n}\right](1 \leqq i, j, k \leqq h)$. In the second case, we can find an element $d \in K\left[\left[x_{1}, \cdots, x_{n}\right]\right]$ which is a unit in $\mathfrak{r}$ and which is such that $d a_{i j k} \in K\left[\left[x_{1}, \cdots, x_{n}\right]\right](1 \leqq i, j, k \leqq h)$. In the first case, we denote by $\Im^{\prime}$ the ring generated by $\mathfrak{r}^{\prime}$, by the $h$ elements $d c_{i}(1 \leqq i \leqq h)$, and by $K\left[x_{1}, \cdots, x_{n}\right]$; in the second case, we denote by $\Im^{\prime}$ the ring generated by $\mathfrak{r}^{\prime}$, by the elements $d c_{i}$, and by $K\left[\left[x_{1}, \cdots, x_{n}\right]\right]$. In either case $\Im^{\prime}$ is finite over $\mathfrak{r}^{\prime}$. If $\mathfrak{q}$ is the maximal prime ideal in $\mathfrak{o}, \mathfrak{q} \cap \mathfrak{r}^{\prime}$ is the maximal prime ideal in $\mathfrak{r}^{\prime}$ (because it contains $y_{m+1}, \cdots, y_{n}$ ). It follows that $q \cap \Im^{\prime}$ is a maximal prime ideal in $\mathfrak{Y}^{\prime}$. The ring of quotients $\mathfrak{b}^{\prime}$ of $\mathfrak{g} \cap \mathfrak{Y}^{\prime}$ with respect to $\mathfrak{Y}^{\prime}$ is clearly contained in $\boldsymbol{0}$. We shall prove that $\boldsymbol{o}^{\prime}=\mathbf{0}$. In the first case, $d$ does not belong to $q \cap K\left[x_{1}, \ldots, x_{n}\right]$; in the second case, $d$ does not belong to $q \cap K\left[\left[x_{1}, \cdots, x_{n}\right]\right]$. It follows that, in either case, $d^{-1}$ belongs to $D^{\prime}$. We

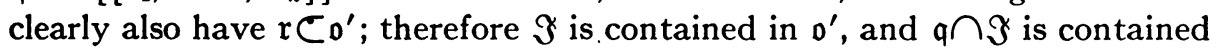

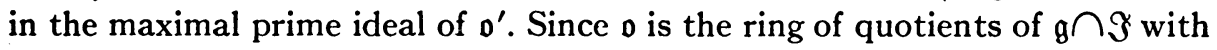
respect to $\Im$, we have $\mathfrak{o}=\mathfrak{o}^{\prime}$. It follows that $\mathfrak{r}^{\prime}$ is a nucleus of $\mathfrak{b}$, with the intermediary ring $\Im^{\prime}$.

Lemma 6. Let $\mathrm{o}$ be a ring which admits a nucleus and let $\mathfrak{p}$ be a prime ideal in $\mathbf{0}$. Then $\mathrm{o} / \mathrm{p}$ admits a nucleus.

By Lemma 5, we can find a nucleus $r$ of $o$ such that $\mathfrak{p} \cap \mathfrak{r}$ is the ideal generated in $\mathfrak{r}$ by a subset of some special system of parameters in $r$. It follows immediately that $\mathfrak{r} /(\mathfrak{p} \cap \mathfrak{r})$ is either of the type $\mathfrak{r}(r ; K)$ or $\mathfrak{\mathfrak { r }}(n, r ; K)$ with suitable $n, r, K$. Let $\Im$ be an intermediary ring of $o$ with respect to $r$. Then $\Im /(\mathfrak{p} \cap \Im)$ is finite over $\mathfrak{r} /(\mathfrak{p} \cap \mathfrak{r})$. Let $\mathfrak{q}$ be the maximal prime ideal in $\mathfrak{o}$. Then $(q \cap \Im) /(\mathfrak{p} \cap \Im)$ is a prime ideal in $\Im /(\mathfrak{p} \cap \Im)$ and contains the maximal prime ideal $(\mathfrak{q} \cap \mathfrak{r}) /(\mathfrak{p} \cap \mathfrak{r})$ of $\mathfrak{r} /(\mathfrak{p} \cap \mathfrak{r})$; it is therefore a maximal prime ideal in $\Im /(p \cap \Im)$. Any element of 0 may be written in the form $a / b$, with $a, b \in \Im$, $b \notin q$. Let $a^{*}, b^{*}$ be the residue classes of $a, b$ modulo $\mathfrak{p}$. Then $b^{*}$ does not belong to $(q \cap \Im) /(\mathfrak{p} \cap \Im)$ and the residue class of $a / b$ is $a^{*} / b^{*}$. It follows that 
$\mathfrak{D} / \mathfrak{p}$ is the ring of quotients of $(q \cap \Im) /(\mathfrak{p} \cap \Im)$ with respect to $\Im /(\mathfrak{p} \cap \Im)$. Therefore, $\mathfrak{r} /(\mathfrak{p} \cap \mathfrak{r})$ is a nucleus of $\mathfrak{o} / \mathfrak{p}$.

LEMMA 7. Let $\mathfrak{o}$ be a ring which admits a nucleus $\mathfrak{r}$ and let $\Im$ be an intermediary ring of $\mathrm{o}$ with respect to $\mathrm{r}$. Let $\mathfrak{p}$ be a prime ideal in $\mathrm{o}$. Let $\Omega$ be the subring of the ring of quotients of $\Im$ which is generated by $\mathfrak{r}_{\mathfrak{p} \cap \mathfrak{x}}$ and $\Im$. Then $\Re$ is a semi-local ring and $p_{\Re}=(p \cap \Im) \Omega$ is a maximal prime ideal in $\Re$. Let $\phi$ and $\phi^{\prime}$ be the natural mappings of $\mathrm{o}$ into $\mathrm{D}_{\mathfrak{p}}$ and of $\Omega$ into $\Omega_{\mathfrak{p}_{\mathfrak{R}}}$; then, there exists an isomorphism $\theta$ of $\Omega_{\mathfrak{p}_{\mathfrak{R}}}$ with $\mathfrak{D}_{\mathfrak{p}}$ such that $\theta\left(\phi^{\prime}(x)\right)=\phi(x)$ for every $x \in \Im$. If $\mathfrak{p} \cap \mathfrak{r}$ can be generated by some subset of a special system of parameters in $\mathfrak{r}$, then $\theta\left(\phi^{\prime}\left(\mathfrak{r}_{\mathfrak{p} \cap \mathfrak{r}}\right)\right)$ is a nucleus in $\mathrm{D}_{\mathfrak{p}}$ and $\theta\left(\phi^{\prime}(\Omega)\right)$ is an intermediary ring for $\mathrm{D}_{\mathrm{p}}$ with respect to this nucleus.

Let $\mathfrak{w}$ be the intersection of all primary components of the zero ideal in $\Im$ which are contained in $\mathfrak{p} \cap \Im$. Since $\mathfrak{b}$ is the ring of quotients with respect to $\Im$ of a maximal prime ideal in $\Im$, wo is the intersection of the primary components of the zero ideal in $\mathfrak{D}$ which are contained in $\mathfrak{p}$; wo is therefore the kernel of $\phi\left({ }^{8}\right)$, and $\mathfrak{o}_{\mathfrak{p}}$ is the ring of quotients of $\mathfrak{p} / \mathfrak{m o}$ with respect to $\mathfrak{o} / \mathfrak{m o}$. The ring $\Omega$ may also be considered as the ring of quotients with respect to $\Im$ of the complement $S$ of $\mathfrak{p} \cap \mathfrak{r}$ with respect to $r$. It follows that $\mathfrak{w} \Omega$ is the intersection of all primary components of the zero ideal in $\Omega$ which are contained in $p_{\Omega}$; the ring $\Omega_{\mathfrak{p}_{\Omega}}$ is the ring of quotients of $p_{\Re} / \mathfrak{w} \Omega$ with respect to $\Omega / \mathfrak{w} \Omega$. Let $Z$ be the ring of quotients of $\Im$, and therefore also of $\mathbf{o}$. The ring of quotients of $\Im / \mathfrak{w}$ may be identified with $Z / \mathfrak{w} Z$. The rings $\mathfrak{o} / \mathfrak{w o}, \Omega / \mathfrak{w} \Omega$ may be identified with subrings of $Z / \mathfrak{w} Z$. The residue class modulo $\mathfrak{w}$ of an element of $S$ does not belong to $\mathfrak{p} / \mathfrak{m o}$ and is therefore a unit in $\mathfrak{o} / \mathfrak{w o}^{\circ}$; it follows immediately that $\Re / \mathfrak{m} \Omega \subset \mathfrak{D}_{\mathfrak{p}}$. The ring $\Omega$ is clearly finite over $\mathfrak{r}_{\mathfrak{p} \cap \mathfrak{r}}$; it follows that $\Omega$ is a semi-local ring (Proposition 3, L.R., $\S I I$, p. 694). Since $p_{\Omega}$ contains the maximal prime ideal of $\mathfrak{r}_{\mathfrak{p} \cap \mathfrak{r}}$, it is a maximal prime ideal in $\Omega$. We have $(\mathfrak{p} \cap \Im) / \mathfrak{w} \subset \mathfrak{p} / \mathfrak{w o}$; it follows that $p_{\Re} / \mathfrak{w} \Omega \subset(\mathfrak{p} / \mathfrak{w o}) \mathfrak{p}_{\mathfrak{p}}$ and that $\Omega_{\mathfrak{p} \Omega} \subset \mathfrak{p}_{\mathfrak{p}}$. On the other hand, an element $a \in 0$ may be written in the form $b / c$, where $b$ and $c$ are in $\Im$ and $c \notin p \cap \Im$. The residue class $a^{*}$ of $a$ modulo $\mathfrak{w}$ is equal to $b^{*} / c^{*}$, where $b^{*}$ and $c^{*}$ are the residue classes of $b$ and $c$ modulo $\mathfrak{w}$ (since $c$ is not a zero divisor in $\mathfrak{b}, c$ does not belong to any of the prime divisors of $\mathfrak{w}$, and it follows that $c^{*}$ is not a zero divisor); moreover, $c^{*}$ does not belong to $(\mathfrak{p} \cap \Im) / \mathfrak{w}$. It follows immediately that $a^{*} \in \Omega_{\mathfrak{p}_{\mathfrak{R}}}$ whence $\mathfrak{o} / \mathfrak{w o}_{\mathfrak{D}} \subset \Omega_{\mathfrak{p}_{\mathfrak{R}}}$. If $a \in \mathfrak{p}$, we have $b \in \mathfrak{p} \cap \Im$ and $a^{*} \in \mathfrak{p}_{\Re} / \mathfrak{w} \Omega$; we conclude easily that $\mathfrak{o}_{\mathfrak{p}}=\Omega_{\mathfrak{p}_{\Re}}$. When we have identified $\mathfrak{D} / \mathfrak{w}$ and $\Omega / \mathfrak{w} \Omega$ with subrings of $Z / \mathfrak{w} Z$, the elements $\phi(x), \phi^{\prime}(x)$ (where $x \in \Im$ ) have been identified with the same element, namely with the residue class of $x$ modulo $\mathfrak{w} Z$. The first part of Lemma 7 is thereby proved.

In order to prove the second part, we observe first that, since no element not equal to 0 in $\mathfrak{r}$ is a zero divisor in $\mathfrak{D}, \mathfrak{r}$ is mapped isomorphically onto

(8) Cf. my paper On the ring of quotients of a prime ideal, Bull. Amer. Math. Soc. vol. 50 (1944) p. 93. 
$\phi(\mathfrak{r})$ by $\phi$. Furthermore, since $\mathfrak{r}$ has no element not equal to 0 in common with any of the prime divisors of $\mathfrak{w}, \phi(\mathfrak{r})$ has no element not equal to 0 in common with any of the prime divisors of the zero ideal in $\mathrm{o} / \mathrm{mo}$, which proves that no element not equal to 0 in $\phi(r)$ is a zero divisor in $o_{p}$. The same holds, of course, for $\theta\left(\phi^{\prime}\left(\mathfrak{r}_{\mathfrak{p} \cap \mathfrak{r}}\right)\right)$, which is the ring of quotients of $\phi(\mathfrak{p} \cap \mathfrak{r})$ with respect to $\phi(\mathfrak{r})$. Moreover, $\theta\left(\phi^{\prime}(\Omega)\right)$ is clearly finite over $\theta\left(\phi^{\prime}\left(\mathfrak{r}_{\mathfrak{p} \cap \mathfrak{r}}\right)\right)$, and we have seen that $\mathfrak{D}_{\mathfrak{p}}$ is the ring of quotients with respect to $\theta\left(\phi^{\prime}(\Omega)\right)$ of a maximal prime ideal in this ring. Assume now that $\mathfrak{p}$ is generated by a subset $\left\{x_{r+1}, \cdots, x_{n}\right\}$ of some special system of parameters in $\mathfrak{r}$. If $\mathfrak{r}$ is of type $\mathfrak{r}(n ; K)$, let $\left\{x_{1}, \cdots, x_{n}\right\}$ be a special system of parameters in $\mathfrak{r}$ which contains $\left\{x_{r+1}, \cdots, x_{n}\right\}$. Then $\mathfrak{r}_{\mathfrak{n} \cap \mathfrak{r}}$ is the ring of quotients of the ideal generated by $x_{r+1}, \cdots, x_{n}$ in $K\left(x_{1}, \cdots, x_{r}\right)\left[x_{r+1}, \cdots, x_{n}\right]$ and is of type $\mathfrak{r}\left(n-r ; K\left(x_{1}, \cdots, x_{r}\right)\right)$. If $\mathfrak{r}$ is of type $\mathfrak{\mathfrak { r }}(n, m ; K)$, let $\left\{x_{1}, \cdots, x_{n}\right\}$ be a special subset in $\mathfrak{r}$ which contains $\left\{x_{r+1}, \cdots, x_{n}\right\}$. Then $\mathfrak{r}_{p} \cap \mathfrak{r}$ is the ring of quotients of the ideal generated by $x_{r+1}, \cdots, x_{n}$ in $K\left[\left[x_{1}, \cdots, x_{n}\right]\right]$ and is of type $\overline{\mathfrak{r}}(n, r ; K)$. Lemma 7 is now completely proved.

Lemmas 5, 6 and 7 show that the class of rings which admit nuclei is closed with respect to the operations of constructing factor rings or rings of quotients of prime ideals. We shall now see that this class is also closed with respect to the operation of completion.

LEMMA 8. Let $\mathfrak{r}$ and $\Im$ be rings which satisfy the following conditions: $\mathfrak{r}$ is a subring of $\Im$ and $\Im$ is finite over $\mathfrak{r}$; no element not equal to 0 in $\mathfrak{r}$ is a zero divisor in $\Im ; \mathfrak{r}$ is a regular local ring. Then $\Im$ is a semi-local ring; the adherence $\overline{\mathfrak{r}}$ of $\mathfrak{r}$ in a completion $\bar{\Im}$ of $\Im$ is a completion of $\mathfrak{r}$; no element not equal to 0 in $\overline{\mathfrak{r}}$ becomes a zero divisor in $\bar{\Im} ; \bar{\Im}$ is generated by $\overline{\mathfrak{r}}$ and $\mathfrak{\Im}$. Let $Z$ be the ring of quotients of $\Im$, and let $R$ and $\bar{R}$ be the fields of quotients of $\mathfrak{r}$ and $\overline{\mathfrak{r}}$. Then the ring of quotients of $\bar{\Im}$ is $Z_{\bar{R}}$ (where $Z$ is regarded as a hypercomplex system over $R$ ).

Our first group of assertions follows immediately from Proposition 7, L.R., $\S I I$, p. 699 , if we observe that a completion of $\mathfrak{r}$, being a regular ring, has no zero divisor not equal to 0 . We can take a base of $Z / R$ which is composed of elements of $\Im$; by Proposition 7, L.R., $§ I I$, p. 699, the elements of this base are linearly independent with respect to $\overline{\mathfrak{r}}$; it follows immediately that the ring of quotients of $\bar{\Im}$ is $Z_{\bar{R}}$.

Lemma 9. Let $\mathfrak{o}$ be a ring which admits a nucleus $\mathfrak{r}$. Then the adherence $\overline{\mathfrak{r}}$ of $\mathfrak{r}$ in a completion $\overline{\mathrm{D}}$ of $\mathfrak{D}$ is a completion of $\mathfrak{r} ; \overline{\mathrm{D}}$ is finite over $\overline{\mathfrak{r}}$ and no element not equal to 0 in $\overline{\mathrm{r}}$ becomes a zero dirisor in $\overline{\mathrm{D}} ; \overline{\mathrm{r}}$ is a nucleus for $\overline{\mathrm{D}}$, and $\overline{\mathrm{D}}$ is generated by $\overline{\mathfrak{r}}$ and $\mathrm{D}$. The prime divisors of the zero ideal in $\overline{\mathrm{D}}$ all have the same dimension as $\overline{\mathbf{D}}$. If the zero ideal in $\mathrm{D}$ is an intersection of prime ideals, the same holds for the zero ideal in $\overline{0}$.

Let $\Im$ be an intermediary ring for $\mathfrak{D}$ with respect to $\mathrm{r}$; let $\bar{\Im}$ be a completion of $\Im$ and let $\overline{\mathfrak{r}}_{1}$ be the adherence of $\mathfrak{r}$ in $\bar{\Im}$. If $\mathfrak{o}$ is the ring of quotients of 
the maximal prime ideal $\mathfrak{M}$ in $\Im$, there corresponds to $\mathfrak{M}$ an idempotent $\epsilon$ in $\bar{\Im}$ which has the following property: there exists an isomorphism $\theta$ of $\bar{\Im} \epsilon$ (considered as a ring with unit element $\epsilon$ ) with $\overline{0}$ such that $\theta(x \epsilon)=x$ for $x \in \mathcal{Y}$ (cf. Proposition 8, L.R., §II, p. 700)( $\left.{ }^{9}\right)$. It is clear that $\theta\left(\tilde{\mathfrak{r}}_{1} \epsilon\right)$ is the adherence of $\mathfrak{r}$ in $\overline{\mathfrak{b}}$; since no element not equal to 0 in $\overline{\mathfrak{r}}_{1}$ is a zero divisor in $\bar{\Im}, \overline{\mathfrak{x}}_{1}$ is isomorphic to $\overline{\mathfrak{x}}_{1} \epsilon$. Moreover, no element not equal to 0 in $\overline{\mathfrak{r}}_{1} \epsilon$ is a zero divisor in $\bar{\Im} \epsilon$. In fact, assume that $(x \epsilon)(y \epsilon)=0, x \in \overline{\mathfrak{x}}_{1}, x \neq 0, y \in \bar{\Im}$; then $x y \epsilon=0$, whence $y \epsilon=0$, which proves our assertion.

If $\mathfrak{r}$ is of type $\mathfrak{r}(n ; K)$, we have $\overline{\mathfrak{r}}=K\left[\left[x_{1}, \cdots, x_{n}\right]\right]$, with $x_{1}, \cdots, x_{n}$ analytically independent over $K$ and $\overline{\mathfrak{r}}$ is of type $\overline{\mathfrak{r}}(n, 0 ; K)$. If $\mathfrak{r}$ is of type $\overline{\mathfrak{r}}(n, m ; K)$, we have $\overline{\mathfrak{r}}=K\left(\left(x_{1}, \cdots, x_{m}\right)\right)\left[\left[x_{m+1}, \cdots, x_{n}\right]\right]$ and $\overline{\mathfrak{r}}$ is of type $\tilde{\mathfrak{r}}\left(n-m, 0 ; K\left(\left(x_{1}, \cdots, x_{m}\right)\right)\right)$.

Let $\overline{\mathfrak{n}}$ be a prime divisor of the zero ideal in $\overline{\mathfrak{b}}$; in virtue of what we have proved already, we have $\overline{\mathfrak{n}} \cap \overline{\mathfrak{r}}=0$. Since $\overline{\mathfrak{b}}$ is finite over $\overline{\mathfrak{r}}, \overline{\mathfrak{b}} / \overline{\mathfrak{n}}$ is finite over a ring which is isomorphic to $\overline{\mathrm{r}}$; making use of the corollary to Proposition 7 , L.R., §III, p. 703, we conclude that $\operatorname{dim} \overline{\mathfrak{b}} / \overline{\mathfrak{n}}=\operatorname{dim} \overline{\mathfrak{r}}=\operatorname{dim} \overline{\mathfrak{b}}$. Let $R$ and $\bar{R}$ be the field of quotients of $\mathfrak{r}$ and of $\overline{\mathfrak{r}}_{1}$. If $\mathfrak{r}$ is of type $\mathfrak{r}(n ; K)$, we have $R=K\left(x_{1}, \cdots, x_{n}\right)$ and $\bar{R}=K\left(\left(x_{1}, \cdots, x_{n}\right)\right)$; in this case, we have proved elsewhere $\left({ }^{10}\right)$ that $\bar{R}$ is separably generated over $R$. If $\mathfrak{r}$ is of type $\overline{\mathfrak{r}}(n, m ; K)$, we have $R=K\left(\left(x_{1}, \cdots, x_{n}\right)\right)$ and $\bar{R}=K\left(\left(x_{1}, \cdots, x_{m}\right)\right)\left(\left(x_{m+1}, \cdots, x_{n}\right)\right)$. We shall prove that, in this case also, $\bar{R}$ is separably generated over $R$. Let $p$ be the characteristic of $K$, which we assume to be not equal to 0 . If a finite extension of $R$ is contained in $R^{1 / p} / R$, it is also contained in $K^{1 / p}\left(\left(x_{1}^{1 / p}, \cdots, x_{n}^{1 / p}\right)\right) / R$. In order to prove that $\bar{R} / R$ is separably generated, it will be sufficient to prove that $\left[K^{1 / p}\left(\left(x_{1}^{1 / p}, \cdots, x_{n}^{1 / p}\right)\right): R\right]=\left[K^{1 / p}\left(\left(x_{1}^{1 / p}\right.\right.\right.$, $\left.\left.\left.\cdots, x_{m}^{1 / p}\right)\right)\left(\left(x_{m+1}^{1 / p}, \cdots, x_{n}^{1 / p}\right)\right): \bar{R}\right]$. (Observe that the numbers which occur on both sides of this formula are finite, because $\left[K: K^{p}\right]$ is finite; this is the only place where we need this assumption on $K$.) The left side of our formula is equal to $p^{n}\left[K^{1 / p}: K\right]$, because we know that $K\left(\left(x_{1}, \cdots, x_{n}\right)\right)$ is separably generated over $K$. For the same reason, we have $\left[K^{1 / p}\left(\left(x_{1}^{1 / p}, \cdots, x_{m}^{1 / p}\right)\right)\right.$ $\left.: K\left(\left(x_{1}, \cdots, x_{m}\right)\right)\right]=p^{m} \cdot\left[K^{1 / p}: K\right]$. Remembering that $K\left(\left(x_{1}, \cdots, x_{m}\right)\right)$ $\cdot\left(\left(x_{m+1}, \cdots, x_{n}\right)\right)$ is separably generated over $K\left(\left(x_{1}, \cdots, x_{m}\right)\right)$, we see that the right side of the formula to be proved is equal to $p^{n-m} p^{m}\left[K^{1 / p}: K\right]$, that is, also to the left side.

Having proved that the extension $\bar{R} / R$ is in every case separably generated, we observe that, if the zero ideal in $D$ is an intersection of prime ideals, the ring of quotients $Z$ of $\mathrm{D}$ is a semi-simple hypercomplex system over $R$. It follows that $Z_{\bar{R}}$ is also semi-simple( $\left.{ }^{11}\right)$. The ring of quotients of $\bar{D}$ is isomor-

(9) Proposition 8 is proved in L.R. only in the case of a ring without zero divisors. It has been extended to the general case in my paper quoted in note $\left(^{8}\right)$ above.

$\left.{ }^{10}\right)$ Cf. On some properties of ideals in rings of power series, Trans. Amer. Math. Soc. vol. 55 (1944) Proposition 5, p. 147.

(11) Cf. the paper quoted in note ${ }^{(10)}$ above, Proposition 3, p. 69. 
phic to $Z_{\bar{R}} \epsilon$, which is again semi-simple. It follows that the zero ideal in $\overline{\mathfrak{D}}$ is an intersection of prime ideals.

Remark. We have $\operatorname{dim} \overline{\mathfrak{D}}=\operatorname{dim} \overline{\mathfrak{r}}$, whence $\operatorname{dim} \mathfrak{D}=\operatorname{dim} \mathfrak{r}$. We have therefore also proved that, if a ring $\mathrm{o}$ admits a nucleus $\mathfrak{r}$, then $\mathrm{o}$ has the same dimension as $\mathfrak{r}$.

Since the class of rings which admit nuclei is closed under the operations by which we have defined the class $(B)$, we see that every ring of the class (B) admits a nucleus. In particular, every ring in $(\$)$ is a local ring. We may therefore use the term geometric local ring to denote a ring of the class $\mathbb{B}$.

THEOREM 1. If $\mathrm{o}$ is a geometric local ring, the zero ideals in $\mathrm{o}$ and in a completion $\overline{\mathrm{D}}$ of $\mathrm{D}$ are intersections of prime ideals which all have the same dimension as $\mathbf{0}$. If $\mathfrak{p}$ is a prime ideal in $\mathrm{o}$, then $\mathrm{p} \overline{\mathrm{D}}$ is an intersection of prime ideals in $\overline{\mathrm{D}}$.

Consider the class $(S)$ of rings which admit nuclei and in which the zero ideal is an intersection of prime ideals. If $\mathfrak{D} \in\left(\mathfrak{S}^{\prime}\right.$, then a completion of $\mathfrak{o}$ belongs to $\mathcal{B}^{\prime}$ by Lemma 9 . Let $\mathfrak{p}$ be a prime ideal in $\mathfrak{D}$; then $\mathfrak{D} / \mathfrak{p}$ has a nucleus by Lemma 6 , and the zero ideal in $\mathfrak{D} / \mathfrak{p}$ is prime. It follows that $\mathfrak{p} p \mathfrak{p}$ is in $\mathcal{B S}^{\prime}$. The ring $o_{\mathfrak{p}}$ has a nucleus by Lemma 7 . If $\mathfrak{n}_{1}, \cdots, \mathfrak{n}_{\mathfrak{g}}$ are the prime divisors of the zero ideal in $\mathfrak{o}$ which are contained in $\mathfrak{p}$, and if $\mathfrak{w}=\mathfrak{n}_{1} \cap \ldots \cap \mathfrak{n}_{g}$, then the zero ideal in $\mathfrak{o} / \mathfrak{w}$ is the intersection of the prime ideals $\mathfrak{n}_{i} / \mathfrak{w}(1 \leqq i \leqq g)$. Since every primary component of the zero ideal in $D$ is prime, $o_{\mathfrak{p}}$ is a ring of quotients of $\mathfrak{o} / \mathfrak{w}$, which proves that the zero ideal in $\mathfrak{o}_{\mathfrak{p}}$ is an intersection of prime ideals, and that $\mathrm{o}_{\mathfrak{p}} \in \mathcal{S}^{\prime}$. It follows that ${ }^{\prime} \subset \subset \mathcal{S}^{\prime}$, which proves the first part of Theorem 1. The second part follows immediately if we observe that $\mathfrak{D} / \mathfrak{p}$ is a geometric local ring whose completion is $\overline{\mathfrak{b}} / \mathfrak{p} \overline{\mathfrak{D}}$ (by Proposition 5, L.R., §II, p. 699)..

THEOREM 2. Let $\mathfrak{o}$ be a geometric local ring, and let $\mathfrak{p}$ be a prime ideal in $\mathrm{o}$. Then we have $\operatorname{dim} \mathfrak{o} / \mathfrak{p}+\operatorname{dim} o_{\mathfrak{p}}=\operatorname{dim} \mathbf{0}$.

Let $\mathfrak{r}$ be a nucleus of $\mathfrak{o}$ such that $\mathfrak{p} \cap \mathfrak{r}$ is generated by a subset of some special system of parameters in $\mathfrak{r}$ (cf. Lemma 5). Then $\mathfrak{r} /(\mathfrak{p} \cap \mathfrak{r})$ is a nucleus of $\mathfrak{o} / \mathfrak{p}$, and $\mathfrak{o}_{\mathfrak{p}}$ admits a nucleus isomorphic with $\mathfrak{r}_{\mathfrak{p} \cap r}$ (cf. proofs of Lemmas 6 and 7). The remark which follows the proof of Lemma 9 shows that $\operatorname{dim} \mathfrak{o} / \mathfrak{p}=\operatorname{dim} \mathfrak{r} /(\mathfrak{p} \cap \mathfrak{r}), \operatorname{dim} \mathfrak{o}_{\mathfrak{p}}=\operatorname{dim} \mathfrak{r}_{\mathfrak{p} \cap \mathfrak{r}}, \operatorname{dim} \mathfrak{v}=\operatorname{dim} \mathfrak{r}$. If $\mathfrak{r}$ is of type $\mathfrak{r}(n ; K)$, let $\left\{x_{1}, \cdots, x_{n}\right\}$ be a special system of parameters in $\mathfrak{r}$ which contains a set of generators $\left\{x_{r+1}, \cdots, x_{n}\right\}$ for $\mathfrak{p} \cap \mathfrak{r}$. Then $\mathfrak{r} /(\mathfrak{p} \cap \mathfrak{r})$ is isomorphic with the ring of quotients with respect to $K\left[x_{1}, \cdots, x_{r}\right]$ of the ideal generated by $x_{1}, \cdots, x_{r}$ in this ring and $\mathfrak{r}_{\mathfrak{p} \cap r}$ is the ring of quotients of the ideal generated by $x_{r+1}, \cdots, x_{n}$ in $K\left(x_{1}, \cdots, x_{r}\right)\left[x_{r+1}, \cdots, x_{n}\right]$. It follows that $\operatorname{dim} \mathfrak{r} /(\mathfrak{p} \cap \mathfrak{r})=r, \operatorname{dim} \mathfrak{r}_{\mathfrak{p} \cap \mathfrak{r}}=n-r, \operatorname{dim} \mathfrak{r}=n$, and our formula is proved in this case. Assume now that $\mathfrak{r}$ is of type $\mathfrak{r}(n, m ; K)$ and let $\left\{x_{1}, \cdots, x_{n}\right\}$ be a special set in $\mathfrak{r}$ which contains a system of generators $\left\{x_{r+1}, \cdots, x_{n}\right\}$ of $\mathfrak{p} \cap \mathfrak{r}$. Then $\mathfrak{r} /(\mathfrak{p} \cap \mathfrak{r})$ is isomorphic to the ring of quotients of the ideal generated 
by $x_{m+1}, \cdots, x_{r}$ in $K\left[\left[x_{1}, \cdots, x_{r}\right]\right]$ and $\mathfrak{r}_{p \cap r}$ is the ring of quotients of the ideal generated by $x_{r+1}, \cdots, x_{n}$ in $K\left[\left[x_{1}, \cdots, x_{n}\right]\right]$. Therefore we have now $\operatorname{dim} \tilde{\mathfrak{r}} /(\mathfrak{p} \cap \mathfrak{r})=r-m, \operatorname{dim} \mathfrak{r}_{\mathfrak{p} \cap \mathfrak{r}}=n-r$, and $\operatorname{dim} \mathfrak{r}=n-m$. Theorem 2 is thereby proved.

Corollary. Let o be a geometric local ring, and let $\left\{x_{1}, \cdots, x_{n}\right\}$ be a system of parameters in $\mathrm{o}$. Let $m$ be an integer less than $n$, and let $\mathfrak{p}$ be a minimal prime divisor of the ideal generated by $x_{m+1}, \cdots, x_{n}$ in $\mathfrak{o}$. Then $\mathfrak{p}$ is of dimension $m$; the residue classes of $x_{1}, \cdots, x_{m}$ modulo $p$ form a system of parameters in $\mathrm{o} / \mathrm{p}$; the images of $x_{m+1}, \cdots, x_{n}$ by the natural homomorphism of $\mathrm{D}$ onto $\mathrm{D}_{\mathrm{p}}$ form a system of parameters in $\mathrm{D}_{\mathfrak{p}}$.

Let $\phi$ be the natural homomorphism of $\mathfrak{o}$ into $\mathfrak{o}_{\mathfrak{p}}$. We know that the prime ideals in $\mathfrak{o}_{\mathfrak{p}}$ correspond in a one-to-one way to the prime ideals in $\mathfrak{D}$ which are contained in $\mathfrak{p}$. It follows immediately that the only prime ideal in $\mathfrak{D}_{\mathfrak{p}}$ to contain $\phi\left(x_{m+1}\right), \cdots, \phi\left(x_{n}\right)$ is the maximal ideal $\phi(\mathfrak{p}) \mathfrak{o}_{\mathfrak{p}}$ of $\mathfrak{o}_{\mathfrak{p}}$. Therefore, we have $\operatorname{dim} \mathfrak{o}_{\mathfrak{p}} \leqq n-m$. If a prime ideal in $\mathfrak{o}$ contains $\mathfrak{p}$ and $x_{1}, \cdots, x_{m}$, it is the ideal of nonunits. It follows that the only prime ideal in $\mathfrak{o} / \mathfrak{p}$ to contain the residue classes of $x_{1}, \cdots, x_{m}$ is the ideal of nonunits in $\mathrm{o} / \mathrm{p}$. Therefore we have $\operatorname{dim} \mathfrak{o} / \mathfrak{p} \leqq m$. It follows from Theorem 2 that $\operatorname{dim} \mathfrak{o} / \mathfrak{p}=m$, $\operatorname{dim} \mathfrak{o}_{\mathfrak{p}}=n-m$. The other assertions contained in the corollary follow immediately.

2. Equidimensional rings and the notion of multiplicity. In L.R. we have defined $\left({ }^{12}\right)$ the notion of the multiplicity of a complete local ring $D$ without any zero divisor not equal to 0 with respect to a system of parameters in $\mathbf{D}$. We shall now generalize this definition so as to include certain local rings which have zero divisors or are not complete.

Definition 1. A complete local ring $\mathrm{o}$ is said to be equidimensional if the prime divisors of the zero ideal in $\mathrm{o}$ have all the same dimension as $\mathrm{o}$ itself. An arbitrary local ring is said to be equidimensional if its completion is equidimensional.

We shall first give an equivalent characterization of complete equidimensional rings.

PROPOSITION 1. Let $\mathrm{D}$ be a complete local ring. Let $K$ be a basic field of $\mathrm{D}$ and let $\left\{u_{1}, \cdots, u_{r}\right\}$ be a system of parameters in $\mathrm{D}$. The following two statements are equivalent: (1) $\mathrm{o}$ is equidimensional; (2) no element not equal to 0 in $K\left[\left[u_{1}, \cdots, u_{r}\right]\right]$ is a zero divisor in $\mathrm{D}$.

Set $\mathfrak{r}=K\left[\left[u_{1}, \cdots, u_{r}\right]\right]$. Let $\mathfrak{n}$ be a prime divisor of the zero ideal in $\mathfrak{o}$. Since $\mathfrak{o}$ is a finite $\mathfrak{r}$-module, $\mathfrak{o} / \mathfrak{n}$ is a finite module over $\mathfrak{r} /(\mathfrak{n} \cap \mathfrak{r})$; it follows that $\mathfrak{o} / \mathfrak{n}$ has the same dimension as $\mathfrak{r} /(\mathfrak{n} \cap \mathfrak{r}$ ) (cf. corollary to Proposition 7 , \$III, L.R., p. 703). It follows that, if 0 is equidimensional, we have

(12) Cf. Definition 1, L.R., §IV, p. 707. 
$\operatorname{dim} \mathfrak{r} /(\mathfrak{n} \cap \mathfrak{r})=r$ for every $\mathfrak{n}$, whence $\mathfrak{n} \cap \mathfrak{r}=\{0\}$ (cf. Proposition 6, §III, L.R., p. 703) and therefore $\mathfrak{r}$ does not contain any zero divisor in $\mathfrak{o}$ not equal to 0. Conversely, if (2) holds, we have $\mathfrak{n} \cap \mathfrak{r}=\{0\}$ for every $\mathfrak{n}$, whence $\operatorname{dim} \mathfrak{o} / \mathfrak{n}=r$, and $\boldsymbol{D}$ is equidimensional.

If $\mathfrak{D}$ is a complete equidimensional local ring, the zero ideal in $\boldsymbol{D}$ has no imbedded prime divisor. In fact, if $\mathfrak{n}_{1}, \mathfrak{n}_{2}$ are two distinct prime ideals in $\mathfrak{D}$ such that $\mathfrak{n}_{1} \subset \mathfrak{n}_{2}$, the ring $\mathfrak{D} / \mathfrak{n}_{2}$ is isomorphic to $\left(\mathfrak{o} / \mathfrak{n}_{1}\right)\left(\mathfrak{n}_{2} / \mathfrak{n}_{1}\right)$, whence $\operatorname{dim} \mathfrak{o} / \mathfrak{n}_{2}<\operatorname{dim} \mathfrak{o} / \mathfrak{n}_{1}$ by Propositon 6, §III, L.R., p. 703. It follows that there exists a unique irredundant representation $\{0\}=q_{1} \cap \cdots \cap q_{h}$ of the zero ideal in $\boldsymbol{D}$ as intersection of primary ideals. We denote by $\mathfrak{n}_{k}$ the associated prime ideal of $\mathfrak{q}_{k}$ and by $l_{k}$ the length of $\mathfrak{q}_{k}(1 \leqq k \leqq h)$. The residue classes $u_{1, k}, \cdots, u_{r, k}$ of $u_{1}, \cdots, u_{r}$ modulo $\mathfrak{n}_{k}$ form a system of parameters in $\mathfrak{o} / \mathfrak{n}_{k}$ (cf. Proposition 1, L.R., §III, p. 701). Denote by $e_{k}$ the multiplicity of $\mathfrak{o} / \mathfrak{n}_{k}$ with respect to this system of parameters, and set

$$
e=\sum_{k=1}^{h} e_{k} l_{k}
$$

DEFINITION 2. The number e defined by formula (1) is called the multiplicity of $\mathrm{D}$ with respect to the system of parameters $\left\{u_{1}, \cdots, u_{r}\right\}$.

Proposition 2. The notation being as in Proposition 1, let e be the multiplicity of $\mathfrak{o}$ with respect to $\left\{u_{1}, \cdots, u_{r}\right\}$. Then we have $\left[0: K\left[\left[u_{1}, \cdots, u_{r}\right]\right]\right]$ $=e[0 / \mathfrak{m}: K]$, where $\mathrm{m}$ is the ideal of nonunits in 0 .

The ring of quotients $S$ of $\boldsymbol{D}$ is a hypercomplex system over the field of quotients $K\left(\left(u_{1}, \cdots, u_{r}\right)\right)$ of $K\left[\left[u_{1}, \cdots, u_{r}\right]\right]$, and we have $\left[\subseteq: K\left(\left(u_{1}, \cdots, u_{r}\right)\right)\right]$ $=\left[0: K\left[\left[u_{1}, \cdots, u_{r}\right]\right]\right]$. The prime ideals in $\subseteq$ are the ideals $\mathfrak{n}_{k} \subseteq(1 \leqq k \leqq h) ;$ the primary component of $\mathfrak{n}_{k} \subseteq$ in the zero ideal of $\subseteq$ is $q_{k} \subseteq$ and the length of $\mathfrak{q}_{k} \subseteq$ is $l_{k}$ (cf. Lemmas 2, 3, L.R., §I, p. 691). Making use of Lemma 1, L.R., $\S I V$, p. 705 , we obtain the formula $\left[\Im: K\left(\left(u_{1}, \cdots, u_{r}\right)\right)\right]=\sum_{k=1}^{h} l_{k}\left[\Im / \mathfrak{n}_{k} \subseteq\right.$ $\left.: K\left(\left(u_{1}, \cdots, u_{r}\right)\right)\right]$. But $\subseteq / \mathfrak{n}_{k} \subseteq$ is isomorphic to the field of quotients of $\mathrm{o} / \mathfrak{n}_{k}, \quad$ whence $\left[\Im / \mathfrak{n}_{k} \subseteq: K\left(\left(u_{1}, \cdots, u_{r}\right)\right)\right]=\left[\mathfrak{o} / \mathfrak{n}_{k}: K\left[\left[u_{1, k}, \cdots, u_{r, k}\right]\right]\right]$ $=e_{k}\left[\left(\mathfrak{o} / \mathfrak{n}_{k}\right) /\left(\mathfrak{m} / \mathfrak{n}_{k}\right): K\right]=e_{k}[\mathfrak{o} / \mathfrak{m}: K]$ (cf. L.R., Theorem 2, p. 706). Proposition 2 is thereby proved.

Definition 3. Let $\mathrm{o}$ be an equidimensional local ring, and let $\left\{u_{1}, \cdots, u_{r}\right\}=\Sigma$ be a system of parameters in 0 . The multiplicity of $\mathrm{o}$ with respect to $\Sigma$ is defined to be equal to the multiplicity of the completion $\overline{0}$ of $\mathrm{o}$ with respect to $\Sigma$; this multiplicity will be denoted by $e(0 ; \Sigma)$ or $e\left(0 ; u_{1}, \cdots, u_{r}\right) .(O b-$ serve that $\Sigma$ is a system of parameters in $\overline{\mathrm{D}}$ by Proposition 2, L.R., §III, p. 701.)

It follows from Theorem $1, \S 1$, p. 11 that a geometric local ring 0 is equidimensional (because a completion of $\mathfrak{D}$ is also a geometric local ring). Let $\mathfrak{n}_{1}, \cdots, \mathfrak{n}_{o}$ be the prime divisors of the zero ideal in $\mathfrak{o}$, and let $\left\{u_{1}, \cdots, u_{r}\right\}$ be a system of parameters in $\mathbf{0}$. We denote by $u_{i k}$ the residue 
class of $u_{i}$ modulo $\mathfrak{n}_{k}(1 \leqq i \leqq r, 1 \leqq k \leqq g)$. The elements $u_{1 k}, \cdots, u_{r k}$ form a system of parameters in $\mathfrak{o} / \mathfrak{n}_{k}$, and we have

$$
e\left(0 ; u_{1}, \cdots, u_{r}\right)=\sum_{k=1}^{o} e\left(0 / \mathfrak{n}_{k} ; u_{1 k}, \cdots, u_{r k}\right) .
$$

In fact, let $\overline{0}$ be a completion of $\boldsymbol{D}$. Then $\mathfrak{n}_{k} \overline{\boldsymbol{D}}$ is an intersection of prime ideals $\overline{\mathfrak{n}}_{k l}$ in $\overline{\mathfrak{D}}\left(1 \leqq l \leqq m_{k}\right)$, and $\overline{\mathfrak{b}} / \overline{\mathfrak{n}}_{k l}$ has the same dimension as $\overline{\mathfrak{b}}$, which proves that each $\overline{\mathfrak{n}}_{k l}$ is a prime divisor of the zero ideal in $\overline{\mathbf{j}}$. Conversely, let $\overline{\mathfrak{n}}$ be any prime divisor of the zero ideal in $\overline{\mathrm{o}}$. Since no nonzero divisor in $\mathrm{o}$ becomes a zero divisor in $\overline{\mathbf{D}}$ (Proposition 6, §II, L.R., p. 699), we see that the elements of $\overline{\mathfrak{n}} \cap \mathfrak{o}$ are zero divisors in $\mathfrak{D}$, whence $\overline{\mathfrak{n}} \cap \mathfrak{D}=\mathfrak{n}_{k}$ for some $k$; it follows that $\overline{\mathfrak{n}}$ is one of the ideals $\overline{\mathfrak{n}}_{k l}$. Let $u_{i, k l}$ be the residue class of $u_{i}$ modulo $\overline{\mathfrak{n}}_{k l}$; it follows immediately from Definition 3 that

$$
\begin{aligned}
e\left(\mathfrak{o} ; u_{1}, \cdots, u_{r}\right) & =\sum_{k=1, l=1}^{o m_{k}} e\left(\overline{\mathfrak{o}} / \overline{\mathfrak{n}}_{k l} ; u_{1, k l}, \cdots, u_{r, k l}\right), \\
e\left(\mathfrak{o} / \mathfrak{n}_{k} ; u_{1, k}, \cdots, u_{r, k}\right) & =\sum_{l=1}^{m_{k}} e\left(\overline{\mathfrak{o}} / \overline{\mathfrak{n}}_{k l} ; u_{1, k l}, \cdots, u_{r, k l}\right) .
\end{aligned}
$$

Formula (2) is thereby proved.

Now, let $\mathfrak{p}$ be a prime ideal in a geometric local ring 0 . Then $\mathfrak{o} / \mathfrak{p}$ is a geometric local ring. Let $\bar{j}$ be a completion of $\mathfrak{D}$; we know that $\bar{p} \bar{b}$ is an intersection of prime ideals $\mathfrak{p}_{1}, \ldots, \mathfrak{p}_{\mathfrak{g}}$ which are all of the same dimension as $\mathfrak{p}$ (cf. corollary to Theorem $2, \S 1$, p. 11). Let $u_{1}, \cdots, u_{s}$ be elements of $\mathrm{o}$ whose residue classes $u_{1}^{*}, \cdots, u_{\varepsilon}^{*}$ modulo $\mathfrak{p}$ form a system of parameters in $\mathfrak{o} / \mathfrak{p}$. Then the residue classes $u_{1, k}^{*}, \cdots, u_{s, k}^{*}$ of $u_{1}, \cdots, u_{s}$ modulo $\mathfrak{p}_{k}$ form a system of parameters in $\bar{\delta} / \mathfrak{p}_{k}$, and we have clearly

$$
e\left(\mathfrak{o} / \mathfrak{p} ; u_{1}^{*}, \cdots, u_{s}^{*}\right)=\sum_{k=1}^{o} e\left(\overline{\mathfrak{D}} / \mathfrak{p}_{k} ; u_{1, k}^{*}, \cdots, u_{s, k}^{*}\right) .
$$

Proposition 3. Let o be an equidimensional local ring, and let $\left\{u_{1}, \cdots, u_{r}\right\}$ and $\left\{v_{1}, \cdots, v_{r}\right\}$ be two systems of parameters in $\mathrm{o}$. If the ideals $\sum_{i=1}^{r} \mathrm{o} u_{i}$ and $\sum_{i=1}^{r} 0 v_{i}$ are equal, we have $e\left(0 ; u_{1}, \cdots, u_{r}\right)=e\left(0 ; v_{1}, \cdots, v_{r}\right)$.

Let $\overline{\mathrm{o}}$ be a completion of $\mathrm{o}$. If $\sum \mathrm{o} u_{i}=\sum \mathrm{o} v_{i}$, we have also $\sum \overline{\mathrm{o}} u_{i}=\sum \overline{\mathrm{o}} v_{i}$. Let $\mathfrak{n}$ be a prime divisor of the zero ideal in $\overline{0}$, and let $u_{i}^{*}, v_{i}^{*}$ be the residue classes of $u_{i}, v_{i}$ respectively modulo $\mathfrak{n}$. Then we have $\sum(\overline{\mathrm{o}} / \mathfrak{n}) u_{i}^{*}=\sum(\overline{\mathrm{o}} / \mathfrak{n}) v_{i}^{*}$. Proposition 3 therefore follows from Proposition 1, L.R., §IV, p. 707.

TheOREM 3. Let $\mathrm{o}$ be an equidimensional local ring, and let $\left\{u_{1}, \cdots, u_{r}\right\}$ be a system of parameters in $\mathrm{D}$. Then, the following two assertions are equivalent: (1) 0 is regular and $u_{1}, \cdots, u_{r}$ form a regular system of parameters in 0 ; (2) the number $e\left(0 ; u_{1}, \cdots, u_{r}\right)$ is equal to 1. 
It is obviously sufficient to prove Theorem 3 in the case where $\mathfrak{o}$ is complete. Let then $K$ be a basic field of 0 , and let $v_{1}, \cdots, v_{d}$ be elements of 0 whose residue classes modulo the maximal prime ideal $\mathfrak{m}$ form a base of $\mathfrak{o} / \mathfrak{m}$ with respect to $K$. If (1) holds, we have $D=\sum_{i=1}^{d} K\left[\left[u_{1}, \cdots, u_{r}\right]\right] v_{i}$ (cf. proof of Proposition 4, L.R., §II, p. 695), whence $\left[0: K\left[\left[u_{1}, \cdots, u_{r}\right]\right]\right] \leqq d$ $=[\mathrm{o} / \mathrm{m}: K]$. It follows that $e\left(\mathfrak{D} ; u_{1}, \cdots, u_{r}\right) \leqq 1$; since $e\left(\mathfrak{0} ; u_{1}, \cdots, u_{r}\right)$ is an integer greater than 0 , we see that (2) holds. In order to prove the converse, we make use of the theorem of Cohen ${ }^{(13)}$ which says that 0 contains a basic field $K^{\prime}$ which is a complete system of representatives for the residue classes modulo $m$. Assuming that (2) holds, we then have $\left[0: K^{\prime}\left[\left[u_{1}, \cdots, u_{r}\right]\right]\right]$ $=\left[\mathrm{o} / \mathrm{m}: K^{\prime}\right]=1$. This means that $\mathrm{o}$ is contained in $K^{\prime}\left(\left(u_{1}, \cdots, u_{r}\right)\right)$. On the other hand, it is well known that $K^{\prime}\left[\left[u_{1}, \cdots, u_{r}\right]\right]$ is integrally closed in its field of quotients $\left({ }^{14}\right)$; since $\mathfrak{D}$ is finite over $K^{\prime}\left[\left[u_{1}, \cdots, u_{r}\right]\right]$, it follows that $\mathfrak{D}=K^{\prime}\left[\left[u_{1}, \cdots, u_{r}\right]\right]$, which proves that (1) holds.

Remark. We shall have to apply Theorem 3 only in cases where the theorem of Cohen can be established by making use of Proposition 3, L.R., §III, p. 702.

3. Kroneckerian products of complete rings. Let o be a complete semilocal ring. Assume that $\boldsymbol{D}$ contains a field $K$ (with infinitely many elements) which has the following property: if $a$ is the product of the maximal prime ideals in $\mathfrak{b}$, then $\mathfrak{o} / \mathfrak{a}$ is finite over $K$. We shall then say that $K$ is a basic field of $\mathbf{p}$.

Assume that $\mathfrak{D}$ admits a basic field $K$. Then $\mathfrak{D}$ may be considered as a topological vector space over $K$, in general of infinite dimension $\left({ }^{15}\right)$. From the fact that $\mathfrak{o} / \mathfrak{a}$ is finite over $K$, it follows easily that $\mathfrak{o} / \mathfrak{a}^{k}$ is finite over $K$ for every $k$. On the other hand, there exists a definite homomorphism $\omega_{k}$ which maps $\mathfrak{o} / \mathfrak{a}^{k+1}$ onto $\mathfrak{o} / \mathfrak{a}^{k}$ (if $x \in \mathfrak{0}$, the image by $\omega_{k}$ of the residue class of $x$ modulo $\mathfrak{a}^{k+1}$ is the residue class of $x$ modulo $\mathfrak{a}^{k}$ ). It follows that the finitedimensional spaces $\mathfrak{o} / \mathfrak{a}^{k}$ form, together with the mappings $\omega_{k}$, an inverse system of vector spaces $\left({ }^{16}\right)$. Since $\mathfrak{o}$ is complete, we see immediately that the projective limit of this inverse system is $\mathfrak{D}$. It follows that $\mathfrak{D}$, considered as a topological vector space over $K$, is linearly compact and that the Hausdorff countability axioms hold in 0 .

Let $\mathfrak{M}$ be any linearly compact vector space over $K$ in which the countability axioms hold. Then $\mathfrak{M}$ is isomorphic to a product of countably many one-dimensional vector spaces( $\left.{ }^{17}\right)$. A subset $A$ of $\mathfrak{M}$ is said to be null-con-

(13) The proof of this theorem has not yet been published.

(14) This follows for instance from Satz 6 in Krull, Dimensionstheorie in Stellenringe, J. Reine Angew. Math. vol. 179 (1938) p. 209. It is also a consequence of Proposition I, \$7, part II, p. 47.

(15) For the theory of topological vector spaces over a discrete field, cf. Lefschetz, Algebraic Topology, Amer. Math. Soc. Colloquium Publications, 1942, chap. 2, pp. 72-83.

(16) Compare loc. cit. note ( ${ }^{(1)}$ above, 25.4, p. 75.

(17) Compare loc. cit. note ( ${ }^{(1)}$ above, 32.1, p. 83. 
vergent if, given any neighbourhood of 0 in $\mathfrak{M}, A$ contains only a finite number of elements outside this neighbourhood. Such a set is necessarily countable. If we assign to every element $x$ in the set an element $a(x)$ in $K$, then the sum $\sum_{x \in A} a(x) x$ has a meaning in $\mathfrak{M}$. Let $\mathfrak{R}(A)$ be the set of elements which can be represented in the form $\sum_{x \in A} a(x) x$, with a suitable choice of coefficients $a(x)$. We shall see that $\mathfrak{R}(A)$ is a closed subspace of $\mathfrak{M}$. The set of all mappings $x \rightarrow a(x)$ of $A$ into $K$ is clearly a vector space over $K$ and is isomorphic to the product of countably many one-dimensional spaces. If we give to this space its product topology, it becomes a linearly compact space $\mathfrak{A}$. It is clear that $\mathfrak{R}(A)$ is the image of $\mathfrak{A}$ by a continuous linear mapping of $\mathfrak{A}$ into $\mathfrak{M}$. It follows that $\mathfrak{R}(A)$ is linearly compact, and therefore closed.

If an element of $\mathfrak{R}(A)$ can be represented in the form $\sum_{x \in A} a(x) x$ in only one way, then we say that the elements of $A$ are strongly linearly independent over $K$, and that $A$ is a strong base of $\mathfrak{R}(A)$ over $K$. Since every closed vector subspace of $\mathfrak{M}$ is linearly compact, it is isomorphic to a product of countably many one-dimensional spaces, and therefore it has a strong base. If the elements of a set $A$ are strongly linearly independent over $K$, then $A$ can be included in a strong base of $\mathfrak{M}$. In fact, the factor space $\mathfrak{M} / \mathfrak{R}(A)$ is clearly a linearly compact vector space; let $B^{*}$ be a strong base in this space. Let $\left(N_{k}\right)$ be a sequence of neighbourhoods of 0 in $\mathfrak{M}$ whose intersection contains only 0 . Then $B^{*}$ contains only a finite number of elements outside the set $N_{k}+\mathfrak{R}(A) / \mathfrak{R}(A)$. It follows easily that we can find a subset $B$ of $\mathfrak{M}$ such that the residue classes of the elements of $B$ modulo $\mathfrak{R}(A)$ are the elements of $B^{*}$ and that $B$ contains only a finite number of elements outside any given $N_{k}$. We may furthermore assume that no two distinct elements of $B$ are congruent to each other modulo $\mathfrak{R}(A)$. It is then easy to check that $A \cup B$ is a strong base in $\mathfrak{M}$.

Let $\mathrm{o}$ be a complete semi-local ring which admits a basic field $K$. Let $B$ be a strong base of $\mathfrak{D}$ with respect to $K$. Then we may express every $u \in \mathfrak{0}$ in the form $\sum_{x \in B} a(u ; x) x, a(u, x) \in K$. Let $K^{B}$ be the product of as many copies of $K$ as there are elements in $B$, so that to every $x \in B$ there corresponds an $x$-coordinate in $K^{B}$. If we assign to every $u \in 0$ the element of $K^{B}$ whose $x$-coordinate is $a(u ; x)$, we obtain a linear homeomorphism of $\mathrm{D}$ with $K^{B}$. If $x$ and $y$ are any two elements of $B$, then $x y$ may be expressed in the form $\sum_{z \in B} a(x, y, z) z$. Remembering that the multiplication in a semi-local ring is a continuous operation, we see that there corresponds to every $z \in B$ a finite subset $B_{z}$ of $B$ such that $a(x, y, z)=0$ if at least one of the elements $x$ and $y$ does not belong to $B_{\boldsymbol{z}}$.

Let now $\mathfrak{D}^{\prime}$ be another complete semi-local ring which admits a basic field $K^{\prime}$, and assume that $K$ and $K^{\prime}$ are both subfields of some field $M$. Let $B^{\prime}$ be a strong base of $0^{\prime}$ with respect to $K^{\prime}$, and let $x^{\prime} y^{\prime}=\sum_{z^{\prime} \in B^{\prime}} a^{\prime}\left(x^{\prime}, y^{\prime}, z^{\prime}\right) z^{\prime}$ be the formulas which define the multiplication in $\mathrm{D}^{\prime}$. The set $M^{B \times B^{\prime}}$ of all mappings $\left(x, x^{\prime}\right) \rightarrow c\left(x, x^{\prime}\right)$ of $B \times B^{\prime}$ into $M$ is a linearly compact vector space 
over $M$ (it is the product of as many copies of $M$ as there are elements in $\left.B \times B^{\prime}\right)$. If $x \in B, x^{\prime} \in B^{\prime}$, we denote by $x \square x^{\prime}$ the element of $M^{B \times B^{\prime}}$ which assigns 1 to $\left(x, x^{\prime}\right)$ and 0 to every other element of $B \times B^{\prime}$. The elements $x \square x^{\prime}$ clearly form a strong base of $M^{B \times B^{\prime}}$ over $M$.

We shall now define a multiplication in $M^{B \times B^{\prime}}$ by setting

$$
\begin{aligned}
\left(\sum_{x, x^{\prime}} c\left(x, x^{\prime}\right) x \square x^{\prime}\right) & \left(\sum_{x, x^{\prime}} d\left(x, x^{\prime}\right) x \square x^{\prime}\right) \\
= & \sum_{x, x^{\prime}, y, y^{\prime}, z, z^{\prime}} c\left(x, x^{\prime}\right) d\left(y, y^{\prime}\right) a(x, y, z) a^{\prime}\left(x^{\prime}, y^{\prime}, z^{\prime}\right) z \square z^{\prime} .
\end{aligned}
$$

The sextuple sum on the right side has a meaning in virtue of the properties stated above of $a(x, y, z)$ and $a^{\prime}\left(x^{\prime}, y^{\prime}, z^{\prime}\right)$. It is easy to check that this multiplication defines a structure of ring in $M^{B \times B^{\prime}}$. Let $\sum_{x^{\prime}} e\left(x^{\prime}\right) x^{\prime}\left(e\left(x^{\prime}\right) \in K^{\prime}\right)$ be the representation of the unit element of $D^{\prime}$ as linear combination of the elements of $B^{\prime}$. Then the elements of the form $\sum_{x, x^{\prime}} b(x) e\left(x^{\prime}\right) x \square x^{\prime}(b(x) \in K)$ form a subring of our ring, and this subring is isomorphic to $D$ (in the algebriac and in the topological sense). In a similar way, we can construct a subring which is isomorphic with $\mathfrak{D}^{\prime}$. We shall identify these rings with $\mathfrak{o}$ and $\mathfrak{D}^{\prime}$ respectively; let then $\mathfrak{D}$ be the ring which we have constructed. The element

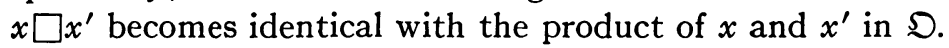

We shall prove that the structure of $\mathfrak{D}$ does not depend upon the choices of the bases $B$ and $B^{\prime}$. We observe first that, if $A^{*}$ is a null-convergent set in $\mathfrak{o}$, it is also null-convergent in $\mathfrak{D}$, and that a symbol of the form $\sum_{u \in A} b(u) u$ (with $b(u) \in K$ ) has the same meaning in $\mathrm{o}$ and in $\mathfrak{D}\left({ }^{18}\right)$. Furthermore, if $A^{\prime}$ is a null-convergent set in $\mathbf{o}^{\prime}$, the set $A A^{\prime}$ composed of the elements $u u^{\prime}\left(u \in A, u^{\prime} \in A^{\prime}\right.$ ) is null-convergent in $\mathfrak{D}$. This being said, let $B^{*}$ and $B^{\prime *}$ be any strong bases in $\mathfrak{D}$ and $\mathfrak{D}^{\prime}$, and let $\mathfrak{D}^{*}$ be the ring which is constructed with the help of $B^{*}$ and $B^{* *}$ in the same way as $\mathfrak{D}$ was constructed with the help of $B$ and $B^{\prime}$. If $x^{*} \in B^{*}, x^{*} \in B^{\prime *}$, we denote by $\left(x^{*} x^{\prime *}\right)^{*}$ the product of $x^{*}$ and $x^{\prime *}$ in $\mathfrak{D}^{*}$ and by $x^{*} x^{\prime *}$ the product of these elements in $\mathfrak{D}$. If we set $\phi\left(\sum_{x^{*}, x^{*}} c\left(x^{*}, x^{*}\right)\left(\left(x^{*} x^{\prime *}\right)^{*}\right)=\sum_{x^{*}, x^{*}} c\left(x^{*}, x^{*}\right) x^{*} x^{\prime *}\right.$, we clearly obtain a continuous homomorphism $\phi$ of $\mathfrak{D}^{*}$ into $\mathfrak{D}$, which is also a linear mapping of $\mathfrak{D}^{*}$ into $\mathfrak{D}$, considered as a vector space over $L$. Furthermore, $\phi$ coincides with the identity on $\mathfrak{D}$ and $\mathfrak{D}^{\prime}$. In a similar way, we can construct a continuous linear homomorphism $\phi^{*}$ of $\mathfrak{D}$ into $\mathfrak{D}^{*}$ which coincides with the identity on $D$ and $\mathfrak{D}^{\prime}$. Then $\phi \circ \phi^{*}$ is a continuous linear homomorphism of $\mathfrak{D}$ into itself which coincides with the identity on $\mathfrak{D}$ and $\boldsymbol{D}^{\prime}$. It follows immediately that $\phi \circ \phi^{*}$ is the identity mapping. The same argument shows that $\phi^{*} \circ \phi$ is the identity mapping of $\mathfrak{D}^{*}$ into itself. It follows that $\phi$ and $\phi^{*}$ are isomorphisms, which proves our assertion.

The ring $\mathfrak{D}$ which was constructed above is called a Kroneckerian product of $\mathrm{D}$ and $\mathrm{D}^{\prime}$ over $M$.

(18) This, because $\mathfrak{D}$ is a subspace of $\mathfrak{D}$. 
Our considerations apply in particular to the case where $D^{\prime}=K^{\prime}$ is a field containing $K$; taking $M=K^{\prime}$, the Kronecker product of $\mathfrak{D}$ and $K^{\prime}$ over $K^{\prime}$ will be denoted by ${ }^{K^{\prime}}\left({ }^{19}\right)$.

Lemma 1. Let $A$ be a null-convergent set of strongly linearly independent elements in $\mathrm{D}$; let $A^{\prime}$ be a null-convergent set of strongly linearly independent elements in $\mathrm{D}^{\prime}$. Then the elements $u u^{\prime}, u \in A, u^{\prime} \in A^{\prime}$ are strongly linearly independent with respect to $M$ in $\mathfrak{D}$.

Lemma 1 follows immediately from the fact that $A$ and $A^{\prime}$ can be imbedded in strong bases of $\mathfrak{D}$ and $\boldsymbol{o}^{\prime}$ respectively.

LEMMA 2. Let $u$ be a nonzero divisor in $\mathrm{o}$ and let $u^{\prime}$ be a nonzero divisor in $\mathrm{o}^{\prime}$. Then $u u^{\prime}$ is not a zero divisor in $\mathfrak{D}$.

Let $B$ and $B^{\prime}$ be strong bases in $\boldsymbol{D}$ and $\boldsymbol{o}^{\prime}$ respectively. Since $u$ is not a zero divisor, the elements $u x, x \in B$, are strongly linearly independent in $\mathbf{D}$. Similarly, the elements $u^{\prime} x^{\prime}, x^{\prime} \in B^{\prime}$, are strongly linearly independent in $\mathfrak{o}^{\prime}$. It follows from Lemma 1 that the elements $u u^{\prime} x x^{\prime}, x \in B, x^{\prime} \in B^{\prime}$, are strongly linearly independent in $\mathfrak{D}$. Since the elements $x x^{\prime}$ form a strong base of $\mathfrak{D}$, it follows that $u u^{\prime}$ is not a zero divisor in $\mathfrak{D}$.

Lemma 3. Let $\mathfrak{u}$ be an ideal in $\mathfrak{o}$, and let $\mathfrak{u}^{\prime}$ be an ideal in $\mathfrak{D}^{\prime}$. Let $\mathfrak{w}$ be the ideal generated by $\mathfrak{u}$ and $\mathfrak{u}^{\prime}$ in $\mathfrak{O}$. Then we have $\mathfrak{w} \cap \mathfrak{D}=\mathfrak{u}, \mathfrak{w} \cap \mathfrak{o}^{\prime}=\mathfrak{u}^{\prime}$. The ring $\mathfrak{O} / \mathfrak{w}$ is a Kroneckerian product of $\mathfrak{o} / \mathfrak{u}$ and $\mathfrak{o}^{\prime} / \mathfrak{u}^{\prime}$ over $M$. In particular, if $K^{\prime}$ is a field containing $K$, then $\mathfrak{u o}_{K^{\prime}} \cap \cap_{\mathfrak{o}}=\mathfrak{u}$ and $\mathfrak{o}_{K^{\prime}} / \mathfrak{u} \mathfrak{o}_{K^{\prime}}=(\mathfrak{o} / \mathfrak{u})_{K^{\prime}}$.

We know that $\mathfrak{u}$ and $\mathfrak{u}^{\prime}$ are closed in $\mathfrak{o}$ and $\mathfrak{o}^{\prime}$ respectively (cf. Lemma 6 , L.R., §II, p. 695). It follows that we can find strong bases $B$ and $B^{\prime}$ of 0 and $\mathfrak{o}^{\prime}$ respectively which contain as subsets strong bases $B_{\mathfrak{u}}$ and $B_{\mathfrak{u}^{\prime}}^{\prime}$ of $\mathfrak{u}$ and $\mathfrak{u}^{\prime}$. We may furthermore assume that $B$ and $B^{\prime}$ contain the unit elements of their respective rings. Let $C$ be the set of elements $x x^{\prime}$ where $x \in B, x^{\prime} \in B^{\prime}$ and either $x \in B_{\mathfrak{u}}$ or $x^{\prime} \in B_{\mathfrak{u}^{\prime}}^{\prime}$. It is clear that the elements of $C$ form a strong base of $\mathfrak{w}$ with respect to $M$, whence $\mathfrak{w} \cap \mathfrak{D}=\mathfrak{u}, \mathfrak{w} \cap \mathfrak{o}^{\prime}=\mathfrak{u}^{\prime}$. Let $B^{*}$ and $B^{\prime *}$ be the. complements of $B_{\mathfrak{u}}$ and $B_{\mathfrak{u}^{\prime}}$ with respect to $B$ and $B^{\prime}$ respectively. The residue classes modulo $\mathfrak{u}$ of the elements of $B^{*}$ form a strong base in $\mathfrak{o} / \mathfrak{u}$; the residue classes modulo $\mathfrak{u}^{\prime}$ of the elements of $B^{\prime *}$ form a strong base in $\mathfrak{o}^{\prime} / \mathfrak{u}^{\prime}$. The complement of $C$ with respect to the set $B B^{\prime}$ of elements of the form $x x^{\prime}$, $x \in B, x^{\prime} \in B^{\prime}$ is the set $B^{*} B^{\prime *}$, and the residue classes of the elements of $B^{*} B^{\prime *}$ modulo $\mathfrak{w}$ form a strong base in $\mathscr{D} / \mathfrak{w}$. It follows immediately that $\mathfrak{D} / \mathfrak{w}$ is a Kroneckerian product of $\mathfrak{o} / \mathfrak{u}$ and $\mathfrak{o}^{\prime} / \mathfrak{u}^{\prime}$ over $M$. The statements contained in the end of Lemma 3 are proved by taking $\mathfrak{u}^{\prime}$ to the zero ideal in $K^{\prime}$.

(19) If $\mathrm{D}$ is a hypercomplex system over $K$, the ring $0_{K^{\prime}}$, as defined here, coincides with the ring which is usually denoted by this symbol. 
We shall now turn our attention to a special kind of complete semi-local rings.

LeMma 4. Assume that there exist finite subsets $\left\{x_{1}, \cdots, x_{n}\right\}$ and $\left\{x_{1}^{\prime}, \cdots, x_{n^{\prime}}^{\prime}\right\}$ of $\mathrm{o}$ and $\mathrm{o}^{\prime}$ respectively with the following properties: $x_{1}, \cdots, x_{n}$ are analytically independent over $K ; x_{1}^{\prime}, \ldots, x_{n}^{\prime}$ are analytically independent over $K^{\prime} ; \mathrm{D}$ is finite over $K\left[\left[x_{1}, \cdots, x_{n}\right]\right] ; \mathfrak{o}^{\prime}$ is finite over $K^{\prime}\left[\left[x_{1}^{\prime}, \cdots, x_{n^{\prime}}^{\prime}\right]\right]$; no element not equal to 0 in $K\left[\left[x_{1}, \cdots, x_{n}\right]\right]$ is a zero divisor in 0 ; no element not equal to 0 in $K^{\prime}\left[\left[x_{1}^{\prime}, \cdots, x_{n^{\prime}}^{\prime}\right]\right]$ is a zero divisor in $\mathrm{o}^{\prime}$. Then $x_{1}, \cdots, x_{n}$, $x_{1}^{\prime}, \ldots, x_{n^{\prime}}^{\prime}$ are analytically independent over $M$ in $\mathfrak{D} ; \mathfrak{D}$ is finite over $M\left[\left[x_{1}, \cdots, x_{n}, x_{1}^{\prime}, \cdots, x_{n^{\prime}}^{\prime}\right]\right]$ and is generated over this ring by $\mathrm{o}$ and $\mathrm{o}^{\prime}$; no element not equal to 0 in $M\left[\left[x_{1}, \cdots, x_{n}, x_{1}^{\prime}, \cdots, x_{n^{\prime}}^{\prime}\right]\right]$ is a zero divisor in $\mathfrak{D}$. We have

$\left[\mathfrak{O}: M\left[\left[x_{1}, \cdots, x_{n}, x_{1}^{\prime}, \cdots, x_{n^{\prime}}^{\prime}\right]\right]\right]$

$$
=\left[\mathrm{o}: K\left[\left[x_{1}, \cdots, x_{n}\right]\right]\right] \cdot\left[\mathrm{o}^{\prime}: K^{\prime}\left[\left[x_{1}^{\prime}, \cdots, x_{n^{\prime}}^{\prime}\right]\right]\right] \text {. }
$$

The first statement follows immediately from Lemma 1 applied to the elements $x_{1}^{e_{1}} \cdots x_{n}^{e_{n}}$ and $x_{1}^{\prime e_{1}} \cdots x_{n^{\prime}}^{\prime e^{\prime} n^{\prime}}$ (with $0 \leqq e_{1}, \cdots, e_{n}, e_{1}^{\prime}, \cdots, e_{n^{\prime}}^{\prime}<\infty$ ). Let $\mathfrak{O}_{1}$ be the subring of $\mathfrak{O}$ generated by $M\left[\left[x_{1}, \cdots, x_{n}, x_{1}^{\prime}, \cdots, x_{n^{\prime}}^{\prime}\right]\right]$ and by $\mathfrak{D}$ and $\mathfrak{D}^{\prime}$. Then $\mathfrak{D}_{1}$ is finite over $M\left[\left[x_{1}, \cdots, x_{n}, x_{1}^{\prime}, \cdots, x_{n^{\prime}}^{\prime}\right]\right]$ and is therefore a complete semi-local ring (Proposition 3, L.R., §II, p. 694). Let $B$ and $B^{\prime}$ be strong bases of $\mathfrak{D}$ and $\mathfrak{D}^{\prime}$ respectively. Then $\mathfrak{D}_{1}$ contains the elements $x x^{\prime}, x \in B, x^{\prime} \in B^{\prime}$. Denote by a the product of the maximal prime ideals in $\mathfrak{o}$, by $\mathfrak{a}^{\prime}$ the product of the maximal prime ideals in $\boldsymbol{o}^{\prime}$, by $\mathfrak{x}$ the ideal generated by $x_{1}, \cdots, x_{n}$ in 0 and by $\mathfrak{r}^{\prime}$ the ideal generated by $x_{1}^{\prime}, \cdots, x_{n^{\prime}}^{\prime}$ in $\mathfrak{o}^{\prime}$. Since every maximal prime ideal of $\mathfrak{o}$ contains $\mathfrak{x}$ and since $\mathfrak{a}$ is also the intersection of the maximal prime ideals of $\mathfrak{o}$, we have $\mathfrak{x} \subset \mathfrak{a}$. The ring $\mathfrak{o} / \mathfrak{a}$ is a hypercomplex system over the field $K\left[\left[x_{1}, \cdots, x_{n}\right]\right] /\left(\mathfrak{x} \cap K\left[\left[x_{1}, \cdots, x_{n}\right]\right]\right)$ $=K$. It follows that the product of the prime ideals in $\mathfrak{o} / \mathfrak{a}$ is nilpotent, whence $\mathfrak{a}^{h} \subset \mathfrak{x}$ for some $h$. In the same way, we see that $\mathfrak{x}^{\prime} \subset \mathfrak{a}^{\prime}, \mathfrak{a}^{\prime h^{\prime}} \subset \mathfrak{x}^{\prime}$ for some $h^{\prime}$. It follows that the powers of $\mathfrak{x}$ form a fundamental system of neighbourhoods of 0 in $\boldsymbol{D}$ and that the powers of $\mathfrak{x}^{\prime}$ form a fundamental system of neighbourhoods of 0 in $\mathfrak{o}^{\prime}$. Let $\mathfrak{X}$ be the ideal generated by $\mathfrak{x}$ and $\mathfrak{x}^{\prime}$ in $\mathfrak{D}_{1}$; then the powers of $\mathfrak{X}$ form a fundamental system of neighbourhoods of 0 in the semilocal ring topology of $\mathfrak{O}_{1}$. It follows immediately that the identity mapping of $\mathfrak{O}_{1}$ into $\mathfrak{D}$ is continuous. We conclude that, if $c\left(x, x^{\prime}\right) \in M$, the symbol $\sum_{x, x^{\prime}} c\left(x, x^{\prime}\right) x x^{\prime}$ has the same meaning in $\mathscr{D}_{1}$ as in $\mathfrak{D}$. It follows immediately. that $\mathfrak{D}_{1}=\mathfrak{D}$.

Let $\left\{u_{1}, \cdots, u_{d}\right\}$ be a maximal system of elements of $\mathfrak{o}$ which are linearly independent with respect to $K\left[\left[x_{1}, \cdots, x_{n}\right]\right]$ and let $\left\{u_{1}^{\prime}, \cdots, u_{d^{\prime}}^{\prime}\right\}$ be a maximal system of elements of $\boldsymbol{o}^{\prime}$ which are linearly independent with respect to $K^{\prime}\left[\left[x_{1}^{\prime}, \cdots, x_{n^{\prime}}^{\prime}\right]\right]$. Then it follows from Lemma 1 that the ele- 
ments $x_{1}^{e_{1}} \ldots x_{n}^{e_{n}} x_{1}^{\prime e_{1}} \ldots x_{n^{\prime}}^{\prime \delta_{n}^{\prime}} u_{i} u_{i^{\prime}}^{\prime}\left(0 \leqq e_{1}, \cdots, e_{n}, e_{1}^{\prime}, \ldots, e_{n^{\prime}}^{\prime}<\infty\right.$, $\left.1 \leqq i \leqq d, 1 \leqq i^{\prime} \leqq d^{\prime}\right)$ are strongly linearly independent over $M$ in $\mathfrak{O}$. On the other hand, there exist elements $\gamma$ and $\gamma^{\prime}$ in $K\left[\left[x_{1}, \cdots, x_{n}\right]\right]$ and $K^{\prime}\left[\left[x_{1}^{\prime}, \ldots, x_{n^{\prime}}^{\prime}\right]\right]$ respectively such that $\gamma_{0} \subset \sum_{i=1}^{d} K\left[\left[x_{1}, \ldots, x_{n}\right]\right] u_{i}$, $\gamma^{\prime} 0^{\prime} \subset \sum_{i^{\prime}-1}^{d^{\prime}} K^{\prime}\left[\left[x_{1}^{\prime}, \cdots, x_{n^{\prime}}^{\prime}\right]\right] u_{i^{\prime}}^{\prime}$. It follows that $\gamma \gamma^{\prime} \mathrm{D} \subset \sum_{i, i^{\prime}=1}^{d, d^{\prime}} M\left[\left[x_{1}, \cdots\right.\right.$, $\left.\left.x_{n}, x_{1}^{\prime}, \cdots, x_{n^{\prime}}^{\prime}\right]\right] u_{\mathfrak{t}} u_{t^{\prime}}^{\prime}$. Let $\xi$ be an element not equal to 0 in $M\left[\left[x_{1}, \cdots, x_{n}\right.\right.$, $\left.\left.x_{1}^{\prime}, \ldots, x_{n^{\prime}}^{\prime}\right]\right]$ and assume that $\xi \zeta=0, \zeta \in D^{\circ}$. We express $\gamma \gamma^{\prime} \zeta$ in the form $\sum_{i, i} z_{i, i} u_{i} u_{i^{\prime}}^{\prime}, z_{i, i} \in M\left[\left[x_{1}, \cdots, x_{n}, x_{1}^{\prime}, \ldots, x_{n^{\prime}}^{\prime}\right]\right]$ and we have $\sum_{i, i^{\prime}} \xi z_{i, i^{\prime}} u_{i} u_{i^{\prime}}^{\prime}=0$, whence $\xi z_{i, i^{\prime}}=0$ for all $\left(i_{i} i^{\prime}\right)$ in virtue of what was proved above. It follows that $z_{i, i^{\prime}}=0$, whence $\gamma \gamma^{\prime} \zeta=0$. But $\gamma \gamma^{\prime}$ is not a zero divisor in $\mathfrak{D}$ (Lemma 2); it follows that $\zeta=0$. Moreover, we see that the $d d^{\prime}$ elements $u_{\mathfrak{i}} u_{\mathfrak{i}^{\prime}}^{\prime}$ form a maximal system of elements of $\mathfrak{D}$ which are linearly independent over $M\left[\left[x_{1}, \cdots, x_{n}, x_{1}^{\prime}, \cdots, x_{n^{\prime}}^{\prime}\right]\right]$. Lemma 4 is completely proved.

Lemma 5. Let $\mathfrak{o}$ be a complete semi-local ring which admits a basic field $K$. Assume that $\mathrm{D}$ contains $n$ elements $x_{1}, \cdots, x_{n}$ which satisfy the conditions formulated in Lemma 4 . Let $K^{*}$ be a field containing $K$. Then $x_{1}, \cdots, x_{n}$ are analytically independent over $K^{*}$ in $\mathrm{o}_{K^{*}} ; \mathfrak{o}_{K^{*}}$ is finite over $K^{*}\left[\left[x_{1}, \cdots, x_{n}\right]\right]$ and is generated by $K^{*}\left[\left[x_{1}, \cdots, x_{n}\right]\right]$ and $\mathrm{o}$. The ring of quotients of $\mathrm{o}_{K^{*}}$ contains $X^{*}=K^{*}\left(\left(x_{1}, \cdots, x_{n}\right)\right)$. If the ring of quotients $Z$ of $\mathrm{D}$ is regarded as a hypercomplex system over $X=K\left(\left(x_{1}, \cdots, x_{n}\right)\right)$, the ring of quotients of $\mathrm{o}_{K} \cdot$ is $Z_{X^{*}}$.

Lemma 5 can be deduced immediately from Lemma 4, applied to the case where $\mathrm{o}^{\prime}=K^{\prime}=K^{*}=M$. The set $\left\{x_{1}^{\prime}, \cdots, x_{n^{\prime}}^{\prime}\right\}$ must be taken here to be the empty set. The fact that the ring of quotients $Z^{*}$ of $\mathfrak{o}_{K^{*}}$ is $Z_{X}$. follows from the facts that $Z^{*}$ is generated by $X^{*}$ and $Z$ and that (in virtue of Lemma 4) $\left[Z^{*}: X^{*}\right]=[Z: X]$.

An idempotent $\epsilon$ contained in a ring 0 is said to be a primitive idempotent in this ring if it cannot be represented as the sum of two idempotents belonging to $D \epsilon$. It follows from Proposition 2, L.R., §II, p. 693 that the primitive idempotents contained in a complete semi-local ring correspond in a one-to-one way to the maximal prime ideals in the ring.

LEMMA 6. The situation being as described in Lemma 5, assume furthermore that $\mathrm{D}$ is a complete local ring. Let $\epsilon$ be a primitive idempotent in $\mathrm{o}_{K^{*}}$. Then $\mathrm{D}_{K} \cdot \epsilon$ is a complete local ring. If $\left\{y_{1}, \cdots, y_{n}\right\}$ is any system of parameters in $\mathrm{D}$, then $\left\{y_{1} \epsilon, \cdots, y_{n} \epsilon\right\}$ form a system of parameters in $0_{K} \cdot \epsilon$, and no element not equal to 0 in $K^{*} \epsilon\left[\left[y_{1} \epsilon, \cdots, y_{n} \epsilon\right]\right]$ is a zero divisor in $\mathrm{D}_{K} \cdot \epsilon$. If $u \in \mathfrak{D}$, the equality $u \epsilon=0$ implies $u=0$. Let $\mathfrak{m}$ be the maximal prime ideal in 0 . If $\mathfrak{m o}_{K} \cdot \epsilon$ is prime, we have $e\left(\mathrm{o}_{K} \cdot \epsilon ; y_{1} \epsilon, \cdots, y_{n} \epsilon\right)=e\left(0 ; y_{1}, \cdots, y_{n}\right)$. The condition that $\mathfrak{m o}_{K^{*}} \epsilon$ should be a prime ideal is certainly satisfied if $K^{*} / K$ is a separably generated extension.

If $\mathfrak{p}^{*}$ is the maximal prime ideal in $\boldsymbol{D}_{K}$. which corresponds to $\epsilon$, the set $p^{*} \epsilon$ contains all nonunits of $\mathfrak{o}_{K^{*}} \epsilon$ and is a prime ideal. It follows that $\mathfrak{o}_{K^{*}} \epsilon$ 
is a complete local ring with $K^{*} \epsilon$ as a basic field. It follows from the assumptions contained in the statement of Lemma 5 that $D$ is an equidimensional local ring, and therefore that $y_{1}, \cdots, y_{n}$ satisfy the same conditions as $x_{1}, \cdots, x_{n}$ (cf. Proposition 1, $\$ 2$, p. 12). By Lemma $5, y_{1}, \cdots, y_{n}$ are analytically independent over $K^{*}$ in $\mathfrak{D}_{K^{*}}, \mathfrak{D}_{K^{*}}$ is finite over $K^{*}\left[\left[y_{1}, \cdots, y_{n}\right]\right]$ and no element not equal to 0 in $K^{*}\left[\left[y_{1}, \cdots, y_{n}\right]\right]$ is a zero divisor in $0_{K^{*}}$. It follows from this last fact that $K^{*}\left[\left[y_{1}, \cdots, y_{n}\right]\right]$ is mapped isomorphically onto $K^{*} \epsilon\left[\left[y_{1} \epsilon, \cdots, y_{n} \epsilon\right]\right]$ by the mapping $y \rightarrow y \epsilon$. Let $y$ be an element not equal to 0 in $K^{*}\left[\left[y_{1}, \cdots, y_{n}\right]\right]$, and assume that $(y \epsilon)(z \epsilon)=0, z \in \mathbb{0}_{K^{*}} ;$ then $y z \epsilon=0$, whence $z \epsilon=0$, which proves that no element not equal to 0 in $K^{*} \epsilon\left[\left[y_{1} \epsilon, \cdots, y_{n} \epsilon\right]\right]$ is a zero divisor in $\mathfrak{D}_{K^{*} \epsilon}$. Since $\mathfrak{D}_{K^{*} \epsilon}$ is finite over $K^{*} \epsilon\left[\left[y_{1} \epsilon, \cdots, y_{n} \epsilon\right]\right]$, the elements $y_{1} \epsilon, \cdots, y_{n} \epsilon$ form a system of parameters in $\mathfrak{D}_{K^{*} \epsilon}$ (cf. corollary to Proposition 7, L.R., §III, p. 703).

We can find a separable algebraic extension $K^{* *} / K^{*}$ of $K^{*}$ which is normal over both $K$ and $K^{*}$ (that is, if an irreducible polynomial with coefficients in $K$ or $K^{*}$ has a linear factor in $K^{* *}$, then it splits into a product of linear factors in $\left.K^{* *}\right)$. It is clear that $0_{K} \cdot=\left(0_{K}\right)_{K} \cdot, D_{K} \cdot \epsilon=\left(0_{K} \cdot \epsilon\right)_{K} \cdot ;$ moreover, $\mathfrak{D}_{K} \cdots \epsilon$ contains some primitive idempotent $\epsilon^{*}$ in $\mathfrak{o}_{K} \cdots$. Let $P$ be the field $\mathfrak{o} / \mathrm{m}$, which will be considered as a hypercomplex system over $K$. It follows from

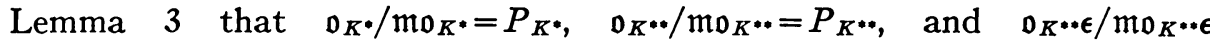

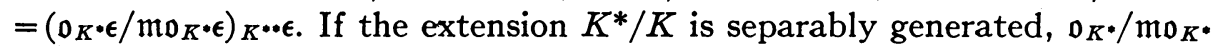
is semi-simple, which proves that $\mathrm{mo}_{K}{ }^{*}$ is an intersection of prime ideals and that $\mathfrak{m o}_{K^{*} \epsilon}$ is prime. If $\mathfrak{m o}_{K^{*} \epsilon}$ is prime, then $\mathfrak{o}_{K} \cdot * \epsilon / \mathfrak{m o}_{K^{* *}}$ is semi-simple (because the extension $K^{* *} / K^{*}$ is separable) and $\mathfrak{m o}_{K} \cdot \epsilon^{*}$ is prime. It follows that it will be sufficient to prove the assertions contained in the end of Lemma 6 in the case where $K^{*}$ is normal over $K$ (because, if this is done, we can apply Lemma 6 to the pairs $\left(\mathfrak{D}, K^{* *}\right)$ and $\left.\left(\mathrm{D}_{K^{*}} \epsilon, K^{* *} \epsilon\right)\right)$.

The idempotents in $P_{K^{*}}$ correspond in a one-to-one way to the irreducible representations of $P$ in $K^{*}\left({ }^{20}\right)$. If $K^{*}$ is normal over $K$, these representations are all conjugate to each other, that is, they can be deduced from each other by automorphisms of $K^{*} / K\left({ }^{21}\right)$. Every automorphism $\sigma$ of $K^{*} / K$ may be extended to an automorphism (also denoted by $\sigma$ ) of $\boldsymbol{o}_{K}$. which leaves invariant the elements of $\mathfrak{o}$. Such an automorphism permutes among themselves the maximal prime ideals in $\mathrm{o}_{K} \cdot$ and therefore also the primitive idempotents

(20) Cf. Jacobson, Theory of rings, Mathematical Surveys, vol. 2, 1943, Theorem 1, chap. 5, p. 93.

(21) This can be seen in the following way. Let $P_{1} / K$ be the largest separable extension of $K$ contained in $P / K$; since $P / P_{1}$ is purely unseparable, a representation of $P$ in $K^{*}$ is uniquely determined by the representation of $P_{1}$ which it induces. Let $\zeta$ be an element of $P_{1}$ such that $P_{1}=K(\zeta)$. The conjugates of $\zeta$ with respect to $K$ fall in a certain number of equivalence classes, two members belonging to the same class if and only if they are conjugate with respect to $K^{*}$. The representations of $P_{1}$ in $K^{*}$ correspond in a one-to-one way to these classes. If $\bar{K}^{*}$ is the algebraic closure of $K^{*}$, the conjugates of $\zeta$ with respect to $K$ can be deduced from each other by automorphisms of $\bar{K}^{*} / K$. Since $K^{*}$ is normal over $K$, these automorphisms map $K^{*}$ upon itself, and our assertion follows from this fact. 
in $0_{K}$. If we observe that the maximal prime ideals in $0_{K} \cdot$ correspond in a one-to-one way to the primitive idempotents in $P_{K^{*}}$, we see that the primitive idempotents in $o_{K^{*}}$ are permuted transitively by the automorphisms $\sigma$. Let $\epsilon_{1}, \cdots, \epsilon_{g}$ be these primitive idempotents (with $\epsilon_{1}=\epsilon$ ). If $u$ is an element of $D$ such that $u \epsilon=0$, we have also $u(\sigma \epsilon)=0$, whence $u\left(\epsilon_{1}+\cdots+\epsilon_{o}\right)=u=0$. Moreover, the numbers $e\left(0_{K} \cdot \epsilon_{i} ; y_{1} \epsilon_{i}, \cdots, y_{n} \epsilon_{i}\right)$ are all equal to each other. Let $e$ be their common value. Then we have (Proposition 2, §2, p. 13)

$$
\left[Z^{*} \epsilon_{i}: K^{*} \epsilon_{i}\left[\left[y_{1} \epsilon_{i}, \cdots, y_{n} \epsilon_{i}\right]\right]\right]=e\left[\mathfrak{o}_{K}{ }^{*} \epsilon_{i} / \mathrm{m}_{i}{ }^{*} \epsilon_{i}: K^{*} \epsilon_{i}\right]
$$

where $Z^{*}$ is the ring of quotients of ${0_{K}}^{*}$ and where $\mathfrak{m}_{i}^{*}$ is the maximal prime ideal which corresponds to $\epsilon_{i}$. If $\mathrm{mo}_{K} \cdot \epsilon$ is prime, then $\mathrm{mo}_{K} \cdot \epsilon_{i}$ is prime for every $i$ and $\mathfrak{m}_{i}^{*} \epsilon_{i}=\mathfrak{m o}_{K^{*}} \epsilon_{i}$. The hypercomplex system $P_{K^{*}}$ is semi-simple, and $\mathrm{O}_{K} \cdot \epsilon_{i} / \mathfrak{m}_{i}^{*} \epsilon_{i}$ is one of the fields, say $P_{i}$, of which $P_{K} \cdot$ is the direct sum. The number $\rho=\left[0_{K} \cdot \epsilon_{i} / \mathrm{m}_{i}^{*} \epsilon_{i}: K^{*} \epsilon_{i}\right]$ does not depend on $i$, and we have $g \rho=\left[P_{K^{*}}: K^{*}\right]=[P: K]=[\mathfrak{o} / \mathfrak{m}: K]$. On the other hand, we have $\left[Z^{*}: K^{*}\right]$ $=\sum_{i=1}^{0}\left[Z^{*} \epsilon_{i}: K^{*} \epsilon_{i}\right]=e g \rho=e[0 / m: K]$. If $Z$ is the ring of quotients of 0 , we have also $\left[Z^{*}: K^{*}\right]=[Z: K]=e\left(0 ; y_{1}, \cdots, y_{n}\right) \cdot[0 / \mathfrak{m}: K]$, whence $e=e\left(0 ; y_{1}, \cdots, y_{n}\right)$. Lemma 6 is thereby proved.

\section{Proof of the theorem of transition.}

Theorem 4 (Theorem of Transition). Let o be a geometric local ring, and let $\mathfrak{p}$ be a prime ideal in $\mathrm{o}$. Let $\overline{\mathrm{b}}$ be a completion of $\mathrm{o}$, and let $\mathfrak{\mathfrak { p }}$ be a minimal prime divisor of $\mathfrak{p} \overline{\mathfrak{D}}$ in $\overline{\mathrm{o}}$. Then $\mathfrak{p} \cap \mathrm{D}=\mathfrak{p}$, and $\mathfrak{p}$ has the same dimension as $\mathfrak{p}$. Denote by $\phi$ and $\bar{\phi}$ the natural homomorphisms of $\mathfrak{o}$ into $\mathrm{o}_{\mathfrak{p}}$ and of $\overline{\mathrm{D}}$ into $\overline{\mathrm{D}}_{\overline{\mathrm{p}}}$ respectively. Let $x_{r+1}, \cdots, x_{n}$ be elements of o such that $\phi\left(x_{r+1}\right), \cdots, \phi\left(x_{n}\right)$ form a system of parameters in ${0_{\bar{p}}}_{\text {. Then }} \phi\left(x_{r+1}\right), \cdots, \phi\left(x_{n}\right)$ form a system of parameters in $\overline{\mathrm{D}}_{\bar{p}}$, and we have

$$
e\left(\overline{\mathfrak{D}}_{\bar{F}} ; \Phi\left(x_{r+1}\right), \cdots, \Phi\left(x_{n}\right)\right)=e\left(\mathfrak{o}_{\mathfrak{p}} ; \phi\left(x_{r+1}\right), \cdots, \phi\left(x_{n}\right)\right) .
$$

Let $\mathfrak{r}$ be a nucleus of $\mathfrak{o}$ such that $\mathfrak{p} \cap \mathfrak{r}$ can be generated by a subset of some special system of parameters in $\mathfrak{r}$ (cf. Lemma $5, \S 1$, p. 6 ). Then $\mathfrak{r}$ is either of the type $\mathfrak{r}(n ; K)$ or $\tilde{\mathfrak{r}}(n, m ; K)$. In the first case, we set $m=0$, so that the dimension of 0 is $n-m$ in any case. We have $\operatorname{dim} o_{\mathfrak{p}}=n-r$, whence by Theorem 2, $\$ 1$, p. 11, $\operatorname{dim} \mathfrak{o} / \mathfrak{p}=r-m$. Since $\operatorname{dim} \mathfrak{r}_{\mathfrak{p} \cap \mathfrak{r}}=\operatorname{dim} \mathfrak{o}_{\mathfrak{p}}$ (cf. Lemma 7, $\S 1$, p. 8 ), we may denote by $\left\{y_{r+1}, \cdots, y_{n}\right\}$ the subset of a special system of parameters $\left\{y_{m+1}, \cdots, y_{n}\right\}$ in $\mathfrak{r}$ which generates $\mathfrak{p} \cap \mathfrak{r}$.

The ideal $\mathfrak{p} / \mathfrak{p} \overline{\mathfrak{D}}$ is a prime divisor of the zero ideal in $\overline{\mathfrak{b}} / \mathfrak{p} \overline{\mathrm{D}}$, which is a completion of $\mathrm{o} / \mathrm{p}$ (cf. Proposition 5, L. R., §II, p. 699). Since no element not equal to 0 in $\mathrm{o} / \mathfrak{p}$ becomes a zero divisor in $\overline{\mathfrak{o}} / \mathfrak{p} \overline{\mathrm{o}}$ (cf. Proposition $6, \mathrm{~L}$. R., $\S I I$, p. 699) we have $(\bar{p} / \mathfrak{p} \overline{\mathfrak{D}}) \cap(\mathfrak{o} / \mathfrak{p})=\{0\}$, whence $\mathfrak{p} \cap \mathfrak{o}=\mathfrak{p}$. Moreover, $\mathfrak{o} / \mathfrak{p}$ is a geometric local ring. It follows from Theorem $1, \S 1$, p. 11 that $\operatorname{dim} \overline{\mathbf{o}} / \mathfrak{p}$ $=\operatorname{dim} \overline{\mathfrak{o}} / \mathfrak{p} \overline{\mathfrak{D}}=\operatorname{dim} \mathfrak{o} / \mathfrak{p}=r-m$. The ideal $\mathfrak{p}$ contains some minimal prime divisor $\tilde{\mathfrak{p}}^{\prime}$ of the ideal generated by $y_{r+1}, \cdots, y_{n}$ in $\overline{\mathfrak{n}}$. By the corollary to Theorem $2, \S 1$, p. 11 , we.have $\operatorname{dim} \mathfrak{p}^{\prime}=r-m$, whence $\mathfrak{p}=\mathfrak{p}^{\prime}$. 
The adherence of $\mathfrak{r}$ in $\overline{\mathfrak{o}}$ is a completion $\overline{\mathfrak{r}}$ of $\mathfrak{r}$ (cf. Lemma 9, 11, p. 9), and $\overline{\mathfrak{D}}$ is finite over $\overline{\mathfrak{r}}$. Because $\overline{\mathfrak{b}} / \mathfrak{p}$ is finite over $\overline{\mathfrak{x}} / \mathfrak{p} \cap \overline{\mathfrak{w}}, \mathfrak{p} \cap \overline{\mathfrak{r}}$ is a prime ideal of dimension $r-m$ in $\overline{\mathfrak{r}}$ and therefore $\mathfrak{p} \cap \overline{\mathfrak{r}}$ is the ideal generated by $y_{r+1}, \cdots, y_{n}$ in $\overline{\mathfrak{r}}$.

Let $\Im$ be an intermediary ring of $\mathfrak{o}$ with respect to $\mathfrak{r}$, and denote by $\bar{\Im}$ a completion of $\Im$. We know $\left.{ }^{22}\right)$ that there exists a primitive idempotent $\epsilon$ in $\bar{\Im}$ with the following property: there exists an isomorphism $\psi$ of $\bar{\delta}$ with $\bar{\Im} \epsilon$ such that $\psi(x)=x \epsilon$ for $x \in \mathfrak{Y}$. The image $\psi(\mathfrak{p})$ of $\mathfrak{p}$ by $\psi$ is a prime ideal in $\overline{\mathfrak{Y}} \epsilon$; the ideal $\mathfrak{p}_{1}$ generated in $\overline{\mathfrak{Y}}$ by $\psi(\mathfrak{p})$ and $1-\epsilon$ is clearly a prime ideal $\mathfrak{p}_{1}$ in $\overline{\mathfrak{Y}}$. The adherence of $\mathfrak{r}$ in $\bar{\Im}$ is a completion $\overline{\mathfrak{r}}_{1}$ of $\mathfrak{r}$, and $\bar{p}_{1} \cap \overline{\mathfrak{r}}_{1}$ is the ideal generated by $y_{r+1}, \cdots, y_{n}$ in $\left.\overline{\mathfrak{r}}_{1}{ }^{23}\right)$. Let $R$ and $\bar{R}$ represent the fields of quotients of $\mathfrak{r}$ and $\overline{\mathfrak{r}}_{1}$ respectively, and let $Z$ and $\bar{Z}$ represent the rings of quotients of $\Im$ and $\bar{\Im}$. Then $\bar{Z}=Z_{\bar{R}}$, where $Z$ is regarded as a hypercomplex system over $R$ (cf. Lemma $8, \S 1$, p. 9).

Denote by the ring of quotients of $\mathfrak{p} \cap \mathfrak{r}$ with respect to $\mathfrak{r}$ and by $\Omega$ the subring of $Z$ which is generated by $\&$ and $\Im$. Denote by $z^{*}$ the ring of quotients of $\mathfrak{p}_{1} \cap \overline{\mathfrak{r}}_{1}$ with respect to $\overline{\mathfrak{r}}_{1}$ and by $\Omega^{*}$ the subring of $\bar{Z}$ which is generated by $\mathbb{8}^{*}$ and $\bar{\Im}$. Then $\Omega$ is finite over $\&$ and $\Omega^{*}$ is finite over $\mathcal{B}^{*}$.

No element not equal to 0 of $\overline{\mathfrak{r}}_{1}$ is a zero divisor in $\bar{\Im}$. It follows that no element not equal to 0 in $\mathbb{z}^{*}$ is a zero divisor in $\Omega^{*}$. Moreover, $\mathbb{z}^{*}$ is a regular local ring of dimension $n-r$.

Denote by $\bar{\Omega}^{*}$ a completion of $\Omega^{*}$ and by $\bar{Z}^{*}$ the ring of quotients of $\bar{\Omega}^{*}$. Then the adherence $\overline{8}^{*}$ of $\dot{s}^{*}$ in $\bar{\Omega}^{*}$ is a completion of $\dot{8}^{*}$, and $\bar{Z}^{*}=\bar{Z}_{S^{*}}$, where $S^{*}$ is the field of quotients of $\overline{\mathrm{g}}^{*}$ (we have $\bar{R} \subset S^{*}$, and $\bar{Z}$ is considered as a hypercomplex system over $\bar{R}$ ).

If $\mathfrak{r}$ is of type $\mathfrak{r}(n ; K)$, we have $\overline{\mathfrak{r}}_{1}=K\left[\left[y_{1}, \cdots, y_{n}\right]\right] ; \mathbb{z}^{*}$ is the ring of quotients of the ideal generated by $y_{r+1}, \cdots, y_{n}$ in $K\left[\left[y_{1}, \cdots, y_{n}\right]\right]$ and $\overline{8}^{*}$ is $K\left(\left(y_{1}, \cdots, y_{r}\right)\right)\left[\left[y_{r+1}, \cdots, y_{n}\right]\right]$. The ring is the ring of quotients of the ideal generated by $y_{r+1}, \cdots, y_{n}$ in $K\left(y_{1}, \cdots, y_{r}\right)\left[y_{r+1}, \cdots, y_{n}\right]$ and $\overline{\mathcal{B}}^{*}$ contains as a subring the ring $K\left(y_{1}, \cdots, y_{r}\right)\left[\left[y_{r+1}, \cdots, y_{n}\right]\right]$ which is a completion $\bar{z}$ of 8 . If $\mathfrak{r}$ is of type $\overline{\mathfrak{r}}(n, m ; K)$, we denote by $\left\{y_{1}, \cdots, y_{n}\right\}$ a special set in $\mathfrak{r}$ which contains $y_{m+1}, \cdots, y_{n}$; we have $\overline{\mathfrak{r}}_{1}=K\left(\left(y_{1}, \cdots, y_{m}\right)\right)\left[\left[y_{m+1}, \cdots, y_{n}\right]\right] ; \mathbb{8}^{*}$ is the ring of quotients of the ideal generated by $y_{r+1}, \cdots, y_{n}$ in $\overline{\mathfrak{r}}_{1}$ and $\overline{\mathfrak{g}}^{*}=K\left(\left(y_{1}, \cdots, y_{m}\right)\right)\left(\left(y_{m+1}, \cdots, y_{r}\right)\right)$ $\left[\left[y_{r+1}, \cdots, y_{n}\right]\right]$. The ring is the ring of quotients of the ideal generated by $y_{r+1}, \cdots, y_{n}$ in $K\left[\left[y_{1}, \cdots, y_{n}\right]\right]$ and $\overline{8}^{*}$ contains as a subring the ring $K\left(\left(y_{1}, \cdots, y_{r}\right)\right)\left[\left[y_{r+1}, \cdots, y_{n}\right]\right]$ which is a completion $\overline{8}$ of 8 .

${ }^{(22)}$ Cf. my paper On the notion of the ring of quotients of a prime ideal, Bull. Amer. Math. Soc. 50 (1944) p. 93, Proposition 5.

$\left.{ }^{23}\right)$ No element not equal to 0 in $\overline{\mathfrak{r}}_{1}$ is mapped upon 0 by the mapping $y \rightarrow y \epsilon$. We have $\overline{\mathfrak{r}}_{1 \epsilon}=\psi(\overline{\mathfrak{r}}),\left(\mathfrak{p}_{1} \cap \overline{\mathfrak{r}}_{1}\right) \epsilon=\psi(\mathfrak{p} \cap \overline{\mathfrak{r}})$ and therefore $\overline{\mathfrak{x}}_{1} / \mathfrak{p}_{1} \cap \overline{\mathfrak{r}}_{1}$ is isomorphic with $\overline{\mathfrak{r}} / \mathfrak{p} \cap \overline{\mathfrak{r}}$ and is of dimension $r-m$. The assertion of the text then follows immediately from the fact that $\overline{\mathfrak{p}}_{1} \cap \overline{\mathfrak{r}}_{1}$ contains $y_{r+1}, \cdots, y_{n}$. 
We see that, in any case, $\overline{8}^{*}$ contains as a subring a completion $\overline{8}$ of 8 . We denote by $S$ the field of quotients of $\overline{8}$. Let $\bar{\Omega}$ be the subring of $\bar{\Omega}^{*}$ which is generated by $\bar{\Omega}$ and $\Omega$. The ring of quotients $Z^{\prime}$ of $\bar{\Omega}$ is generated by $S$ and $Z$; it follows that $Z^{\prime}=Z_{S}$ ( $Z$ being regarded as a hypercomplex system over $R$ and the field of coefficients being extended from $R$ to $S$ ). Since $\bar{\Omega}$ is the subring of $Z_{S}$ which is generated by $\overline{8}$ and $\Omega$, it follows from Lemma $8, \S 1$, p. 9, that $\bar{\Omega}$ is a completion of $\Omega$.

If $\mathfrak{r}$ is of type $\mathfrak{r}(n ; K)$, we set $Y=K\left(y_{1}, \cdots, y_{r}\right), Y^{*}=K\left(\left(y_{1}, \cdots, y_{r}\right)\right)$. If $\mathfrak{r}$ is of type $\tilde{\mathfrak{r}}(n, n ; K)$ we set $Y=K\left(\left(y_{1}, \cdots, y_{r}\right)\right), Y^{*}=K\left(\left(y_{1}, \cdots, y_{m}\right)\right)$ $\cdot\left(\left(y_{m+1}, \cdots, y_{r}\right)\right)$. In either case, $Y$ is a basic field of $\bar{g}$ and $Y^{*}$ is a basic field of $\overline{\mathcal{B}}^{*}$. Furthermore, we have $S=Y\left(\left(y_{r+1}, \cdots, y_{n}\right)\right), S^{*}=Y^{*}\left(\left(y_{r+1}, \cdots, y_{n}\right)\right)$.

We have $Z_{S^{*}}=\left(Z_{S}\right)_{S^{*}}$, and $\bar{\Omega}^{*}$ is the subring of $Z_{S^{*}}$ which is generated by $\overline{\mathbb{Q}}^{*}$ and $\Omega$, or also by $\overline{\mathbb{Q}}^{*}$ and $\bar{\Omega}$. It follows from Lemma $5, \S 3$, p. 20 , that $\bar{\Omega}^{*}=\bar{\Omega}_{Y}$.

The ring $\Omega$ may be considered as the ring of quotients with respect to $\Im$ of the complement of $\mathfrak{p} \cap \mathfrak{r}$ with respect to $\mathfrak{r}$. It follows that ( $\mathfrak{p} \cap \Im) \Omega$ is a prime ideal in $\Omega$; this prime ideal contains the maximal prime ideal $(\mathfrak{p} \cap \mathfrak{r}) \mathbb{8}$ of $\&$ and is therefore a maximal prime ideal in $\Omega$. We conclude that $(p \cap \Im) \bar{\Omega}$ is a maximal prime ideal in the complete semi-local ring $\bar{\Omega}$; there corresponds to this ideal a primitive idempotent $\eta$ in $\bar{\Omega}$ which has the property that $1-\eta$ $\in(\mathfrak{p} \cap \Im) \bar{\Omega}$.

The ring $\Omega^{*}$ may be considered as the ring of quotients with respect to $\bar{\Im}$ of the complement of $\bar{p}_{1} \cap \overline{\mathfrak{x}}_{1}$ with respect to $\overline{\mathfrak{x}}_{1}$. It follows as in the previous case that $\mathfrak{p}_{1} \Omega^{*}$ is a maximal prime ideal in $\Omega^{*}$ and that $\mathfrak{p}_{1} \bar{\Omega}^{*}$ is a maximal prime ideal in $\bar{\Omega}^{*}$. There corresponds to this maximal prime ideal a primitive idempotent $\zeta$ in $\bar{\Omega}^{*}$ such that $1-\zeta \in \bar{p}_{1} \bar{\Omega}^{*}$.

Since $\zeta$ is primitive in $\bar{\Omega}^{*}$, one of the elements $\epsilon \zeta,(1-\epsilon) \zeta$ is 0 . If $\epsilon \zeta$ would be $0, \mathfrak{p}_{1} \bar{\Omega}^{*}$ would contain $\epsilon(1-\zeta)=\epsilon$, which is impossible since $\mathfrak{p}_{1}$ already contains $1-\epsilon$. Therefore, we have $\epsilon \zeta=\zeta$.

We shall prove that $\zeta \eta=\zeta$. Operating as above, we see that it will be sufficient to derive a contradiction from the equality $\zeta \eta=0$. By construction of $\mathfrak{p}_{1}, \mathfrak{p}_{1} \epsilon=\psi(\mathfrak{p})$. Since $\mathfrak{p} \subset \mathfrak{p}$ and $\psi(\mathfrak{p} \cap \Im)=\mathfrak{p} \epsilon$, we have $(\mathfrak{p} \cap \Im) \epsilon \subset \mathfrak{p}_{1}$. Since $\mathfrak{p}_{1}$ also contains $1-\epsilon$, we have $\mathfrak{p} \cap \Im \subset \bar{p}_{1}$, whence $(\mathfrak{p} \cap \Im) \bar{\Omega} \subset \bar{p}_{1} \bar{\Omega}^{*}$ and $1-\eta \in \bar{p}_{1} \bar{\Omega}^{*}$. The argument may then be pursued in the same way as above, when we proved that $\epsilon \zeta=\zeta$.

We can find elements $c_{i} \in \Im(r+1 \leqq i \leqq n)$ which are units in $\mathfrak{D}$ and are such that $c_{i} x_{i} \in \Im$; it is obvious that the multiplicities which the statement of Theorem 3 asserts to be equal are not changed if we replace $x_{i}$ by $c_{i} x_{i}(r+1 \leqq i \leqq n)$. We may therefore assume without loss of generality that the elements $x_{i}$ belong to $\Im$.

Let $\phi^{\prime}$ be the natural homomorphism of $\Omega$ into the ring of quotients

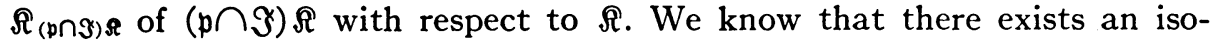
morphism of the completion of $\Omega_{(p \cap \Im) \Omega}$ with $\bar{\Omega} \eta$ which maps $\phi^{\prime}(x)$ upon $x \eta$ 
for $x \in \Omega$. It follows from Lemma $7, \S 1$, p. 8 , that

$$
e\left(\mathfrak{o}_{p} ; \phi\left(x_{r+1}\right), \cdots, \phi\left(x_{n}\right)\right)=e\left(\bar{\Omega} \eta ; x_{r+1} \eta, \cdots, x_{n} \eta\right) .
$$

The equality $\bar{\Omega}^{*}=\bar{\Omega}_{Y^{*}}$ implies that $\bar{\Omega}^{*} \eta=(\bar{\Omega} \eta)_{Y^{*}} ;$ on the other hand, $Y^{*}$ is separably generated over $Y$ (cf. proof of Lemma $9, \S 1$, p. 9). It follows from Lemma $6, \S 3$, p. 20 , that

$$
e\left(\bar{\Omega} \eta ; x_{r+1} \eta, \cdots, x_{n} \eta\right)=e\left(\bar{\Omega}^{*} \zeta ; x_{r+1} \zeta, \cdots, x_{n} \zeta\right) .
$$

Let $\Omega_{\mathfrak{p}_{1} \Omega^{*}}^{*}$ be the ring of quotients of $\tilde{p}_{1} \Omega^{*}$ with respect to $\Omega^{*}$ and let $\phi^{\prime}$ be the natural homomorphism of $\Omega^{*}$ into this ring of quotients. We know that there exists an isomorphism of the completion of $\Omega^{*} \overline{\mathfrak{p}}_{1} \Omega^{*}$ with $\bar{\Omega}^{*} \zeta$ which maps $\Phi^{\prime}(x)$ upon $x \zeta$, for every $x \in \Omega^{*}$. It follows that

$$
e\left(\bar{\Omega}^{*} \zeta ; x_{r+1} \zeta, \cdots, x_{n} \zeta\right)=e\left(\Omega_{\boldsymbol{p}_{1} \Omega * ;}^{*} \Phi^{\prime}\left(x_{r+1}\right), \cdots, \Phi^{\prime}\left(x_{n}\right)\right) .
$$

If some prime ideal in $\bar{\Im}$ is contained in $\bar{p}_{1}$, it must contain one of the elements $\epsilon, 1-\epsilon$ (because $\epsilon(1-\epsilon)=0$ ), and it cannot contain $\epsilon$, because $1-\epsilon \in \bar{p}_{1}$; such a prime ideal must therefore contain $1-\epsilon$. Since $1-\epsilon$ is an idempotent, it belongs also to every primary ideal of $\bar{\Im}$ which is contained in $\mathfrak{p}_{1}$; it follows that $1-\epsilon$ belongs to the kernel of $\phi^{\prime}$ (observe that $\Omega^{*}$ may be considered as the ring of quotients of the complement of $\tilde{p}_{1} \cap \overline{\mathfrak{x}}_{1}$ in $\overline{\mathfrak{x}}_{1}$ with respect to $\bar{\Im})$. We conclude that $\bar{\phi}^{\prime}(\bar{\Im})=\bar{\phi}^{\prime}(\bar{\Im} \epsilon)$, and that, for $x \in \bar{\Im}, \bar{\phi}^{\prime}(x)$ $=\Phi^{\prime \prime}(x \epsilon)$, where $\Phi^{\prime \prime}$ is a homomorphism of $\Im \epsilon$ into $\Omega_{p_{1} \Omega^{*}}^{*}$. The kernel of $\Phi^{\prime \prime}$ is clearly the intersection of all primary components of the zero ideal in $\bar{\Im} \epsilon$ which are contained in $\mathfrak{p}_{1} \epsilon$; this kernel is the image by $\psi$ of the kernel of $\phi$. It follows that there exists an isomorphism $\bar{\theta}$ of $\bar{\phi}(\overline{\mathfrak{D}})$ with $\bar{\phi}^{\prime}(\bar{\Im})$ such that $\bar{\theta}(\bar{\phi}(x))=\bar{\phi}^{\prime}(\psi(x))$ for every $x \in \overline{\mathbf{0}}$. The prime ideals $\bar{\phi}(\mathfrak{p})$ and $\bar{\phi}^{\prime}\left(\tilde{p}_{1}\right)$ correspond to each other by the isomorphism $\bar{\theta}$. It is clear that $\Omega_{\mathfrak{p}_{1} \Omega^{*}}^{*}$ is identical with the ring of quotients of $\bar{\phi}^{\prime}\left(\bar{p}_{1}\right)$ with respect to $\bar{\phi}^{\prime}(\bar{\Im})$; it follows that $\bar{\theta}$ can be extended to an isomorphism of $\overline{\mathfrak{p}}_{\mathrm{p}}$ with $\Omega_{\mathfrak{p}_{1} \Omega^{*}}^{*}$, whence

$$
e\left(\Omega_{\tilde{p}_{1} \Omega \bullet}^{*} ; \Phi^{\prime}\left(x_{r+1}\right), \cdots, \Phi^{\prime}\left(x_{n}\right)\right)=e\left(\overline{\mathfrak{D}}_{\bar{p}} ; \phi\left(x_{r+1}\right), \cdots, \Phi\left(x_{n}\right)\right) .
$$

Theorem 4 is thereby proved.

\section{Proof of the associativity formula.}

THEOREM 5. Let o be a geometric local ring, and let $\left\{x_{1}, \cdots, x_{n}\right\}$ be a system of parameters in $\mathrm{D}$. Let $r$ be an integer less than $n$, and let $p_{1}, \cdots, \mathfrak{p}_{g}$ be the distinct minimal prime divisors of the ideal generated by $x_{r+1}, \cdots, x_{n}$ in 0. Denote by $\phi_{i}$ the natural homomorphism of $\mathrm{D}$ into $\mathrm{D}_{\mathfrak{p}_{i}}$ and by $x_{k, i}$ the residue class of $x_{k}$ modulo $\mathfrak{p}_{i}$. Then we have

$$
e\left(\mathfrak{D} ; x_{1}, \cdots, x_{n}\right)=\sum_{i=1}^{\mathfrak{g}} e\left(\mathfrak{o}_{\mathfrak{p}_{i}} ; \phi_{i}\left(x_{r+1}\right), \cdots, \phi_{i}\left(x_{n}\right)\right) \cdot e\left(\mathfrak{D} / \mathfrak{p}_{i} ; x_{1, i}, \cdots, x_{r, i}\right) .
$$

Let $\overline{\mathrm{D}}$ be a completion of $\mathfrak{D}$. By Theorem $2, \S 1$, p. $11, \mathfrak{p}_{i} \overline{0}$ is an intersection 
of prime ideals $\mathfrak{p}_{i j}\left(i \leqq j \leqq h_{i}\right.$; we assume that $\mathfrak{p}_{i j} \neq \mathfrak{p}_{i j^{\prime}}$ for $\left.j \neq j^{\prime}\right)$ which are all of the same dimension as $\mathfrak{p}$. It follows that $\mathfrak{p}_{i j} \subset \mathfrak{p}_{i j^{\prime}}$ for $j \neq j^{\prime}$; therefore, $\mathfrak{p}_{i j}$ is a minimal prime divisor of $\mathfrak{p}_{i} \overline{\overline{0}}$, and (by Theorem $4, \S 4$, p. 22) we have $\mathfrak{p}_{i j} \cap \mathbb{D}=\mathfrak{p}_{i}$. Let $x_{k, i j}$ be the residue class of $x_{k}$ modulo $\mathfrak{p}_{i j}$; the ideals $\mathfrak{p}_{i j} / \overline{\mathfrak{b}} \mathfrak{p}_{i}$ being the prime divisors of the zero ideal in the completion $\overline{0} / \mathfrak{p}_{i} \overline{0}$ of $0 / \mathfrak{p}_{i}$, we have by formula (2), §2, p. 14,

$$
e\left(\mathbb{0} / \mathfrak{p}_{i} ; x_{1, i}, \cdots, x_{r, i}\right)=\sum_{j=1}^{h_{i}} e\left(\overline{\mathfrak{D}} / \mathfrak{p}_{i j} ; x_{1, i j}, \cdots, x_{r, i j}\right) .
$$

Let $\bar{\phi}_{i j}$ be the natural homomorphism of $\overline{0}$ into the ring $\bar{D}_{\bar{p}} \bar{i}_{j}$; by Theorem 4 , $\$ 4$, p. 22 , we have

$$
e\left(\mathfrak{o}_{\mathfrak{p}_{i}} ; \phi_{i}\left(x_{r+1}\right), \cdots, \phi_{i}\left(x_{n}\right)\right)=e\left(\overline{\mathfrak{o}}_{\mathfrak{j} i j} ; \bar{\phi}_{i j}\left(x_{r+1}\right), \cdots, \bar{\phi}_{i j}\left(x_{n}\right)\right) .
$$

On the other hand, we have by definition $e\left(\mathfrak{0} ; x_{1}, \cdots, x_{n}\right)=e\left(\overline{\mathfrak{b}} ; x_{1}, \cdots, x_{n}\right)$. If $\overline{\mathfrak{q}}$ is any prime ideal in $\overline{\mathfrak{o}}$ which contains $x_{r+1}, \cdots, x_{n}$, then $\overline{\mathfrak{q}} \cap_{\mathrm{o}}$ contains one of the ideals $\mathfrak{p}_{i}$ and therefore $\bar{q}$ contains one of the ideals $\mathfrak{p}_{i j}$. It follows that the ideals $\mathfrak{p}_{i j}$ are all the minimal prime divisors of the ideal generated by $x_{r+1}, \cdots, x_{n}$ in $\overline{\mathbf{D}}$. Therefore, we see that it will be sufficient to prove Theorem 5 in the case where 0 is complete.

Assume that this is the case, and let $K$ be a basic field of 0 . We denote by $\mathfrak{r}$ the ring $K\left[\left[x_{1}, \cdots, x_{n}\right]\right]$ and by $\mathfrak{x}$ the ideal generated by $x_{r+1}, \cdots, x_{n}$ in $\mathfrak{r}$. Then $\mathfrak{o}$ is finite over $\mathfrak{r}$, and therefore $\mathfrak{o} / \mathfrak{p}_{i}$ is finite over $\mathfrak{r} /\left(\mathfrak{p}_{i} \cap \mathfrak{r}\right)$, whence $\operatorname{dim} \mathfrak{r} /\left(\mathfrak{p}_{i} \cap \mathfrak{r}\right)=\operatorname{dim} \mathfrak{o} / \mathfrak{p}_{i}=r$ (by the corollary to Theorem 2, $\$ 1$, p. 11); since $\mathfrak{r} \subset \mathfrak{p}_{i} \cap \mathfrak{r}$, it follows that $\mathfrak{p}_{i} \cap \mathfrak{r}=\mathfrak{r}$.

Let $S$ be the complement of $\mathfrak{x}$ with respect to $\mathfrak{r}$, and let $\Im$ be the ring of quotients of $S$ with respect to $\mathfrak{o}$ (observe that since $S \subset \mathfrak{r}$ and since $\mathrm{o}$ is equidimensional, no element of $S$ is a zero divisor in $\mathfrak{o}$ ). The ring $\Im$ is finite over $\mathfrak{r}_{\mathfrak{r}}$ and is therefore a semi-local ring; the maximal prime ideals in $\Im$ are the prime ideals which contain $r$. Among these maximal prime ideals clearly occur the ideals $\mathfrak{p}_{i} \Im(1 \leqq i \leqq g)$. Conversely, a maximal prime ideal of $\Im$ may be written in the form $q \mathfrak{S}$, where $q$ is a prime ideal in $\mathfrak{o}$ whose intersection with $\mathfrak{r}$ is $\mathfrak{x}$; it follows that $q$ contains one of the ideals $p_{i}$, whence $q \mathfrak{q}=\mathfrak{p}_{i} \mathfrak{T}$ for some $i$.

The ring of quotients $\mathfrak{D}_{\mathfrak{p}_{i}}$ can obviously be identified with $\Im_{\mathfrak{p}_{i} \mathfrak{s}}$. It follows that there exists an isomorphism $\psi_{i}$ of the completion of $\boldsymbol{D}_{\mathfrak{p}_{i}}$ with $\bar{\Im} \epsilon_{i}$ (where $\bar{\Im}$ is a completion of $\Im$ and where $\epsilon_{i}$ is the primitive idempotent in $\bar{\Im}$ which corresponds to the maximal prime ideal $\left.p_{i} \widehat{\Im}\right)$ such that $\psi_{i}\left(\phi_{i}(x)\right)=x \epsilon_{i}$ for every $x \in \mathrm{o}$. Let $Z$ be the ring of quotients of $\mathfrak{D}$, and let $R$ and $\bar{R}$ be the fields of quotients of $\mathfrak{r}$ and of the completion of $\mathfrak{r}_{\mathfrak{r}}$; we know that the ring of quotients of $\bar{\Im}$ is $Z_{\bar{R}}$ ( $Z$ being regarded as a hypercomplex system over $R$; cf. Lemma 8 , $\S 1$, p. 9). We have

$$
[Z: R]=\left[Z_{R}: \bar{R}\right]=\sum_{i=1}^{0}\left[Z_{\bar{R} \epsilon_{i}}: \bar{R}_{\epsilon_{i}}\right]
$$


We have $\bar{R} \epsilon_{i}=K \epsilon_{i}\left(\left(x_{1} \epsilon_{i}, \cdots, x_{r} \epsilon_{i}\right)\right)\left(\left(x_{r+1} \epsilon_{i}, \cdots, x_{n} \epsilon_{i}\right)\right)$. It follows that

$$
\left[Z_{\bar{R}} \epsilon_{i}: \bar{R} \epsilon_{i}\right]=e\left(\bar{\Im}_{\epsilon_{i}} ; x_{r+1} \epsilon_{i}, \cdots, x_{n} \epsilon_{i}\right)\left[\bar{\Im}_{\epsilon_{i}} / \mathfrak{p}_{i} \bar{\Im}_{\epsilon_{i}}: K \epsilon_{i}\left(\left(x_{1} \epsilon_{i}, \cdots, x_{r} \epsilon_{i}\right)\right) .\right.
$$

This first factor in the left side is equal to $e\left(\mathfrak{o}_{p_{i}} ; \phi_{i}\left(x_{r+1}\right), \cdots, \phi_{i}\left(x_{n}\right)\right)$. The ring $\bar{\Im} \epsilon_{i} / \mathfrak{p}_{i} \bar{\Im}_{\epsilon_{i}}$ is a field, which is isomorphic to the field of quotients of $\mathfrak{o} / \mathfrak{p}_{i}$ under an isomorphism which maps $x_{k, i}$ upon the residue class of $x_{k} \epsilon_{i}$ modulo $\mathfrak{p}_{i} \bar{\Im} \epsilon_{i}$. If $\mathfrak{m}$ is the maximal prime ideal of $\mathfrak{o}$, the maximal prime ideal of $\mathfrak{o} / \mathfrak{p}_{i}$ is $m / \mathfrak{p}_{i}$. It follows that the second factor on the right side of our formula is equal to $\left[0 / \mathfrak{p}_{i}: K\left[\left[x_{1, i}, \cdots, x_{r, i}\right]\right]\right]$, that is, also to $e\left(0 / p_{i} ; x_{1, i}, \cdots, x_{r, i}\right)$ $\cdot\left[\left(\mathfrak{o} / \mathfrak{p}_{i}\right) /\left(\mathfrak{m} / \mathfrak{p}_{i}\right): K\right]=e\left(\mathfrak{o} / \mathfrak{p}_{i} ; x_{1, i}, \cdots, x_{r, i}\right)[\mathfrak{o} / \mathfrak{m}: K]$. Remembering that $[Z: R]=e\left(0 ; x_{1}, \cdots, x_{n}\right)[\mathrm{o} / \mathfrak{m}: K]$, we obtain

$$
\begin{aligned}
e\left(0 ; x_{1}, \cdots, x_{n}\right) & {[\mathrm{o} / \mathfrak{m}: K] } \\
& =\sum_{i} e\left(\mathfrak{o}_{i} ; \phi_{i}\left(x_{r, 1}\right), \cdots, \phi_{i}\left(x_{n}\right)\right) e\left(\mathfrak{o} / \mathfrak{p}_{i} ; x_{1, i}, \cdots, x_{r, i}\right)[\mathfrak{o} / \mathfrak{m}: \Re] .
\end{aligned}
$$

Theorem 5 is thereby proved.

CoROLlaRY. Let o be a geometric local ring and let $\left\{x_{1}, \cdots, x_{n}\right\}$ be a system of parameters in 0. Assume that the elements $x_{r+1}, \cdots, x_{n}$ generate a prime ideal $\mathfrak{p}$ in 0. Then we have

$$
e\left(\mathfrak{o} ; x_{1}, \cdots, x_{n}\right)=e\left(\mathfrak{o} / \mathfrak{p} ; x_{1}^{*}, \cdots, x_{r}^{*}\right)
$$

where $x_{1}^{*}, \cdots, x_{r}^{*}$ are the residue classes of $x_{1}, \cdots, x_{r}$ modulo $p$.

Let $\phi$ be the natural homomorphism of $D$ into $o_{p}$. It is clear that $\phi\left(x_{r+1}\right), \cdots, \phi\left(x_{n}\right)$ generate the maximal prime ideal of $\mathfrak{o}_{p}$. The corollary follows therefore from Theorem 3, $\$ 2$, p. 14, and from Theorem 5 above.

\section{PART II}

1. Algebroid varieties. Let $K$ be an algebraically closed field, and let $X_{1}, \cdots, X_{n}$ be $n$ letters. We associate to the ring $K\left[\left[X_{1}, \cdots, X_{n}\right]\right]$ of power series in $X_{1}, \cdots, X_{n}$ an object $E^{n}\left(X_{1}, \cdots, X_{n}\right)$ which we call the $n$-dimensional local space over $K$ with the coordinates $X_{1}, \cdots, X_{n}$. To every prime ideal $\mathfrak{u}$ in $K\left[\left[X_{1}, \cdots, X_{n}\right]\right]$ we associate (in a one-to-one way) an object which we call an algebroid variety in our local space. We say that the algebroid variety and the ideal $\mathfrak{u}$ correspond to each other. In particular, we identify $E^{n}\left(X_{1}, \cdots, X_{n}\right)$ with the algebroid variety which corresponds to the zero ideal. The algebroid variety which corresponds to the ideal generated by $X_{1}, \cdots, X_{n}$ is called the origin of our local space.

If $U$ is the algebroid variety which corresponds to the prime ideal $\mathfrak{u}$, the ring $K\left[\left[X_{1}, \cdots, X_{n}\right]\right] / \mathfrak{u}$ is called the ring of holomorphic functions on $U$; this ring will be denoted by $f(U)$. The ring $f(U)$ is a complete local ring; its dimension is called the dimension of $U$. The dimension of $E^{n}\left(X_{1}, \cdots, X_{n}\right)$ is $n$ and the dimension of the origin is 0 . The residue class modulo $\mathfrak{u}$ of an 
element $F \in K\left[\left[X_{1}, \cdots, X_{n}\right]\right]$ is called the function induced on $U$ by $F$. The field of quotients of $f(U)$ is called the field of meromorphic functions on $U$; this field is denoted by $\mathrm{P}(U)$. The ring of quotients of $\mathfrak{u}$ with respect to $K\left[\left[X_{1}, \cdots, X_{n}\right]\right]$ is called the neighborhood ring of $U$; this ring is denoted by $\mathfrak{R}(U)$ and its completion by $\mathfrak{N}(U)$.

Let $U$, and $V$ be two algebroid varieties in $E^{n}\left(X_{1}, \cdots, X_{n}\right)$, and let $\mathfrak{u}$ and $\mathfrak{v}$ be the corresponding prime ideals. If $\mathfrak{b} \subset \mathfrak{u}$, we say that $U$ is contained in $V$ or that $U$ is a subvariety of $V$; we write $U \subset V$. If $U$ is contained in $V$ but is different from $V$, we say that $U$ is strictly contained in $V$.

Assume that $U$ is a subvariety of $V$. Then $f(U)$ is isomorphic in a natural way to the factor ring of $\mathfrak{f}(V)$ by the prime ideal $\mathfrak{u} / \mathfrak{b}$. The element of $\mathfrak{f}(U)$ which corresponds by this natural isomorphism to the residue class modulo $\mathfrak{u} / \mathfrak{b}$ of an element $\phi \in f(V)$ is called the function induced on $U$ by $\phi$. If $U \subset V \subset W$, the function induced on $U$ by a function $\psi \in f(W)$ is the same as the function induced on $U$ by the function induced by $\psi$ on $V$. If the function induced on $U$ by a function $\phi$ in $f(V)$ is 0 , we say that $\phi$ vanishes on $U$. The set of functions in $\mathfrak{f}(V)$ which vanish on $U$ is $\mathfrak{u} / \mathfrak{b}$; we shall say that $\mathfrak{u} / \mathfrak{b}$ is the prime ideal in $\mathrm{f}(V)$ which corresponds to the subvariety $U$.

Still assuming that $U$ is a subvariety of $V$, we observe that $\mathfrak{N}(U)$ is a subring of $\mathfrak{R}(V)$ and that $\mathfrak{N}(V)$ is the ring of quotients with respect to $\mathfrak{N}(U)$ of the prime ideal $\mathfrak{v} \mathfrak{N}(U)$. We shall say that $\mathfrak{b} \mathfrak{N}(U)$ is the prime ideal which corresponds to $V$ in $\mathfrak{N}(U)$. We know that every prime ideal in $\mathfrak{N}(U)$ can be written in the form $\mathfrak{v} \mathfrak{N}(U)$ where $\mathfrak{v}$ is a prime ideal contained in $\mathfrak{u}$. It follows that there exists a one-to-one correspondence between the prime ideals in $\mathfrak{N}(U)$ and the varieties containing $U$.

The factor ring $\mathfrak{N}(U) / \mathfrak{b} \Re(U)$ is called the neighborhood ring of $U$ with respect to (or on) $V$. This ring is denoted by $\mathfrak{R}_{V}(U)$, and its completion by $\overline{\mathfrak{N}}_{V}(U)$. The prime ideals in this ring are the ideals of the form $\mathfrak{w} \mathfrak{N}(U) / \mathfrak{b} \mathfrak{N}(U)$, where $\mathfrak{w}$ runs over the prime ideals in $K\left[\left[X_{1}, \cdots, X_{n}\right]\right]$ such that $\mathfrak{b} \subset \mathfrak{w} \subset \mathfrak{u}$. They correspond in a one-to-one way to the varieties $W$ which are between $U$ and $V$ (that is, $U \subset W \subset V$ ). It is easy to see that $\mathfrak{R}_{V}(U)$ is isomorphic in a natural way with the ring of quotients with respect to $f(V)$ of the ideal $\mathfrak{u} / \mathfrak{b}$.

Proposition 1. If $U$ is a subvariety of $V$, the dimension of $U$ is at most equal to the dimension of $V$. If these dimensions are equal, we have $U=V$.

This follows immediately from Propositions 1 and 6, L.R., §3, pp. 701, 702.

Proposition 2. Assume that $U$ is a subvariety of $V$, and let $u$ and $v$ be the dimensions of $U$ and $V$. Then $\mathfrak{N}(U)$ and $\mathfrak{N}_{V}(U)$ are equidimensional local rings of respective dimensions $n-u$ and $v-u$.

For $\mathfrak{R}(U)$, this follows immediately from Theorem 2, $\$ 1$, part I, p. 11. For $\mathfrak{N}_{V}(U)$, our assertion follows from the same source if we observe that $\mathfrak{R}_{V}(U)$ is isomorphic with the ring of quotients of $\mathfrak{u} / \mathfrak{b}$ with respect to $\mathfrak{f}(V)$, 
that $f(V)$ is a complete local ring of dimension $v$ with no zero divisor not equal to 0 , and that $\mathfrak{u} / \mathfrak{v}$ is a prime ideal of dimension $u$ in $\mathfrak{f}(V)$.

$A$ convention of notations. When no confusion is possible, we shall denote the ring $K\left[\left[X_{1}, \cdots, X_{n}\right]\right]$ by $K[[X]]$ and the space $E^{n}\left(X_{1}, \cdots, X_{n}\right)$ by $E^{n}(X)$. If we have other series of letters to consider, such as $\left\{X_{1}^{\prime}, \cdots, X_{n^{\prime}}^{\prime}\right\}$ or $\left\{Y_{1}, \cdots, Y_{m}\right\}, \cdots$ we shall use the self-explanatory notations $K\left[\left[X, X^{\prime}\right]\right], K[[X, Y]], \cdots, E^{n+n^{\prime}}\left(X, X^{\prime}\right), E^{n+m}(X, Y), \cdots$ More generally, if we have any finite sequence of quantities $u_{1}, \cdots, u_{k}$ belonging to a complete local ring containing a field $K$, we shall use the notation $K[[u]]$ to represent the ring $K\left[\left[u_{1}, \cdots, u_{k}\right]\right]$ provided no confusion is possible as to the number $k$ of quantities $u$ under consideration.

Product varieties. Let there be given two series of letters $\left\{X_{1}^{(1)}, \ldots, X_{n_{1}}^{(1)}\right\}$ and $\left\{X_{1}^{(2)}, \cdots, X_{n_{2}}^{(2)}\right\}$ with no letter in common. The local space associated with the series of $n_{1}+n_{2}$ letters $\left\{X_{1}^{(1)}, \ldots, X_{n_{1}}^{(1)}, X_{1}^{(2)}, \cdots, X_{n_{2}}^{(2)}\right\}$ is called the product of the local spaces $E^{n_{1}}\left(X^{(1)}\right)$ and $E^{n_{2}}\left(X^{(2)}\right)$ and is denoted by $E^{n_{1}}\left(X^{(1)}\right) \times E^{n_{2}}\left(X^{(2)}\right)$. Let $U_{i}$ be a variety in $E^{n_{i}}\left(X^{(i)}\right)$, defined by a prime ideal $\mathfrak{u}_{i}$ in $K\left[\left[X^{(i)}\right]\right](i=1,2)$, and let $u_{i}$ be the dimension of $U_{i}$. Then we know $\left.{ }^{24}\right)$ that the ideal generated by $\mathfrak{u}^{(1)}$ and $\mathfrak{u}^{(2)}$ in the ring $K\left[\left[X^{(1)}, X^{(2)}\right]\right]$ is prime and of dimension $u_{1}+u_{2}$. The variety in $E^{n_{1}}\left(X^{(1)}\right) X E^{n_{2}}\left(X^{(2)}\right)$ which is associated with this prime ideal is called the product of $U_{1}$ and $U_{2}$ and is denoted by $U_{1} \times U_{2}$. Its dimension is $u_{1}+u_{2}$.

Let $W$ be any variety in $E^{n_{1}}\left(X^{(1)}\right) \times E^{n_{2}}\left(X^{(2)}\right)$, and let $\mathfrak{w}$ be the corresponding prime ideal. Then $\mathfrak{w} \cap K\left[\left[X^{(i)}\right]\right]$ is a prime ideal in $K\left[\left[X^{(i)}\right]\right]$ and defines a variety in $E^{n_{i}}\left(X^{(i)}\right)$. This variety is called the projection of $W$ on $E^{n_{i}}\left(X^{(i)}\right)$ and is denoted by $\operatorname{pr}^{(i)} W$. Using the same notation as above, we have $\operatorname{pr}_{X^{(i)}} U_{1} \times U_{2}=U_{i}$. In fact let $F$ be a power series in the letters $X^{(1)}$ alone which belongs to the ideal generated by $\mathfrak{u}_{1}$ and $\mathfrak{u}_{2}$ in $K\left[\left[X^{(1)}, X^{(2)}\right]\right]$. We have $F=\sum_{k} A_{k} U_{k}^{(1)}+\sum_{l} B_{l} U_{l}^{(2)}$, where each $U^{(i)}$ is in $\mathfrak{u}_{i}$ and $A_{k_{0}} B_{l}$ are power series in the letters $X^{(1)}, X^{(2)}$. Since $U_{l}^{(2)}$ is not a unit, it belongs to the ideal generated by the $n_{2}$ letters $X^{(2)}$ and vanishes identically if we replace these letters by 0 . It follows that

$$
F=\sum_{k} A_{k}\left(X^{(1)}, 0\right) U_{k}^{(1)} \in \mathfrak{u}^{(1)} .
$$

This proves that $\operatorname{pr}^{(1)} U_{1} \times U_{2}=U_{1}$. A similar argument applies to the projection on $E^{n_{2}}\left(X^{(2)}\right)$.

If $W$ is any variety in $E^{n_{1}}\left(X^{(1)}\right) \times E^{n_{2}}\left(X^{(2)}\right)$, we clearly have $W C\left(\operatorname{pr}^{(1)} W\right)$ $\times\left(\operatorname{pr}^{(2)} W\right)(25)$.

${ }^{(24)}$ Cf. my paper Some properties of ideals in rings of power series, Trans. Amer. Math. Soc. vol. 55 (1944) Proposition 12a, p. 168. The assertion on the dimension follows easily from Lemmas 3 and 4, §3, part I, pp. 18, 19.

(25) Note that it is not true in general that $\operatorname{dim}\left(\operatorname{pr}_{X^{(1)}} W\right) \leqq \operatorname{dim} W$. In this respect, algebroid varieties differ from algebraic varieties. 
It should be observed that, when we speak of a series of letters, we do not consider these letters as being arranged in a definite order; that is, we make no distinction between the space $E^{n}\left(X_{1}, \cdots, X_{n}\right)$ and the space $E^{n}\left(X_{\omega(1)}, \cdots, X_{\omega(n)}\right)$ if $\omega$ is a permutation of the set $\{1, \cdots, n\}$. As a consequence, if $U_{i}$ is a variety in $E^{n_{i}}\left(X^{(i)}\right)(i=1,2,3), U_{1} \times U_{2}$ and $U_{2} \times U_{1}$ are different symbols for the same variety, and the same applies to $U_{1} \times\left(U_{2} \times U_{3}\right)$ and $\left(U_{1} \times U_{2}\right) \times U_{3}$. This last variety is also denoted by $U_{1} \times U_{2} \times U_{3}$. Similar remarks would apply to products of more than three varieties.

Copies of a space. Let $\left\{X_{1}, \cdots, X_{n}\right\}$ be a given series of $n$ letters. It is sometimes convenient to introduce a new series of $n$ letters $\left\{X_{1}^{\prime}, \cdots, X_{n}^{\prime}\right\}$ in such a way that the letters $X^{\prime}$ are considered as associated to the letters $X$ in a definite way, $X_{i}^{\prime}$ corresponding to $X_{i}(1 \leqq i \leqq n)$. If this is done, we say that $E^{n}\left(X^{\prime}\right)$ is a copy of the space $E^{n}(X)$. There exists a uniquely determined isomorphism $J$ of $K[[X]]$ with $K\left[\left[X^{\prime}\right]\right]$ which maps $X_{i}$ upon $X_{i}^{\prime}(1 \leqq i \leqq n)$. To every prime ideal $\mathfrak{u}$ in $K[[X]], J$ associates a prime ideal $J(\mathfrak{u})$ in $K\left[\left[X^{\prime}\right]\right]$. It follows that there corresponds to every variety $U$ in $E^{n}(X)$ a variety $U^{\prime}$ in $E^{n}\left(X^{\prime}\right)$ which is called the copy of $U$.

The ring $K\left[\left[X, X^{\prime}\right]\right]$ is identical with $K\left[\left[X, X^{\prime}-X\right]\right]$ and the $n$ quantities $X_{i}^{\prime}-X_{i}$ are analytically independent over $K[[X]]$. It follows that the ideal $\delta$ generated by the elements $X_{i}^{\prime}-X_{i}(1 \leqq i \leqq n)$ in $K\left[\left[X, X^{\prime}\right]\right]$ is prime and defines a variety $\Delta$ in $E^{n}(X) \times E^{n}\left(X^{\prime}\right) ; \Delta$ is called the diagonal of $E^{n}(X) \times E^{n}\left(X^{\prime}\right)$. The ideal $\delta$ has only 0 in common with $K[[X]]$; moreover, if $H\left(X, X^{\prime}\right)$ is any power series in the letters $X, X^{\prime}$, we have

$$
H\left(X, X^{\prime}\right) \equiv H(X, X)(\bmod \delta) .
$$

It follows that the elements of $K[[X]]$ form a complete system of representatives for the residue classes of $K\left[\left[X, X^{\prime}\right]\right]$ modulo $\delta$. We conclude that $f(\Delta)$ is isomorphic with $K[[X]]$ under an isomorphism which maps upon $X_{i}$ the residue class of $X_{i}$ modulo $\delta$. This isomorphism establishes a one-to-one correspondence between the subvarieties of $\Delta$ and the varieties in $E^{n}(X)$. We shall denote by $M^{\Delta}$ the subvariety of $\Delta$ which corresponds to a variety $M$ in $E^{n}(X)$ by this correspondence. It is clear that the conditions $M \subset U, M^{\Delta} \subset U^{\Delta}$ are equivalent. The dimensions of $M$ and $M^{\Delta}$ are equal. If $\mathfrak{m}$ and $\mathfrak{m}^{\Delta}$ are the prime ideals in $K[[X]]$ and $K\left[\left[X, X^{\prime}\right]\right]$ respectively which correspond to $M$ and $M^{\Delta}$, it is clear that $\mathrm{mCm}^{\Delta}$, and it follows immediately from the congruence (1) above that $\mathfrak{m}=\mathfrak{m}^{\Delta} \cap K[[X]]$, and that $\mathfrak{m}^{\Delta}$ is the ideal generated by $m$ and $\delta$ in $K\left[\left[X, X^{\prime}\right]\right]$. In particular we have $M=\operatorname{pr}_{X} M^{\Delta}$.

2. Intersections of algebroid varieties. Let $U$ and $V$ be algebroid varieties in $E^{n}(X)$. We shall say that an algebroid variety $M$ belongs to the intersection of $U$ and $V$ if it is contained in both $U$ and $V$.

Definition 1. A variety $M$ is called a component of the intersection of the varieties $U$ and $V$ if it belongs to this intersection and if no variety strictly con. taining $M$ belongs to the intersection. 
Let $\mathfrak{u}$ and $\mathfrak{v}$ be the prime ideals which correspond to $U$ and $V$ and $K[[X]]$. It is clear that the components of the intersection of $U$ and $V$ correspond to the minimal prime divisors of the ideal generated by $\mathfrak{u}$ and $\mathfrak{v}$. Therefore we have

Proposition 1. The intersection of two algebroid varieties has only a finite number of components. Any variety which is contained in this intersection is contained in one of these components.

THEOREM 1. Let $U$ and $V$ be algebroid varieties of respective dimensions $u$ and $v$ in $E^{n}(X)$. Then the dimension of any component of the intersection of $U$ and $V$ is not less than $u+v-n$.

Let us construct a copy $E^{n}\left(X^{\prime}\right)$ of the space $E^{n}(X)$, and let $V^{\prime}$ be the copy of $V$ in $E^{n}\left(X^{\prime}\right)$. If $M$ is a component of the intersection of $U$ and $V$, then $M^{\Delta}$ belongs to the intersection of $U \times V^{\prime}$ and $\Delta$. It follows that $M^{\Delta}$ is contained in some component of the intersection of $U \times V^{\prime}$ and $\Delta$. This last component, being a subvariety of $\Delta$, can be written in the form $M_{1}^{\Delta}$, where $M_{1}$ is a variety in $E^{n}(X)$ which contains $M$. The inclusion $M_{1} \subset U \times V^{\prime}$ implies that $M_{1} \subset \operatorname{pr}_{X}\left(U \times V^{\prime}\right)=U$ and that $\operatorname{pr}_{X^{\prime}} M_{1} \subset \operatorname{pr}_{X^{\prime}}\left(U \times V^{\prime}\right)=V^{\prime}$, whence $M_{1} \subset V$. Since $M$ was a component of the intersection of $U$ and $V$, we have $M_{1}=M$, and we see that $M^{\Delta}$ is a component of the intersection of $U \times V^{\prime}$ and $\Delta$.

If a subvariety of $U \times V^{\prime}$ contains $M^{\Delta}$ and is such that $X_{1}^{\prime}-X_{1}, \cdots$, $X_{n}^{\prime}-X_{n}$ vanish on it, this variety is contained in $\Delta$ and therefore coincides with $M^{\Delta}$. Let $x_{i}$ and $x_{i}^{\prime}$ be the functions induced by $X_{i}$ and $X_{i}^{\prime}$ on $U \times V^{\prime}$; it follows from what we said that the only prime ideal in $\mathfrak{\Re}_{U \times V^{\prime}}\left(M^{\Delta}\right)$ to contain the $n$ elements $x_{i}^{\prime}-x_{i}(1 \leqq i \leqq n)$ is the maximal prime ideal of this ring, whence $\operatorname{dim} \mathfrak{\Re}_{U \times V^{\prime}}\left(M^{\Delta}\right) \leqq n$. On the other hand, if $M$ is of dimension $d$, we have $\operatorname{dim} \mathfrak{\Re}_{U \times V^{\prime}}\left(M^{\Delta}\right)=u+v-d$, whence $u+v-d \leqq n$, which proves our assertion.

Definition 2. A component of the intersection of two algebroid varieties $U$ and $V$ of respective dimensions $u$ and $v$ is called a proper component of this intersection if it is of dimension $u+v-n$.

It follows immediately from the proof of Theorem 1 that, if $M$ is a proper component of the intersection of $U$ and $V$, the elements $x_{i}^{\prime}-x_{i}(1 \leqq i \leqq n)$ form a system of parameters in $\mathfrak{N}_{U \times V^{\prime}}\left(M^{\Delta}\right)$.

Definition 3. Assume that $M$ is a proper component of the intersection of two algebroid varieties $U$ and $V$ in $E^{n}(X)$. Let $E^{n}\left(X^{\prime}\right)$ be a copy of the space $E^{n}(X)$ and let $V^{\prime}$ be the copy of $V$ in $E^{n}\left(X^{\prime}\right)$. Let $x_{i}, x_{i}^{\prime}$ be the functions induced by $X_{i}, X_{i}^{\prime}$ on $U \times V^{\prime}(1 \leqq i \leqq n)$. Then we set $i(M ; U \cdot V)=e\left(\Re_{U \times V^{\prime}}\left(M^{\Delta}\right)\right.$; $\left.x_{1}^{\prime}-x_{1}, \cdots, x_{n}^{\prime \prime}-x_{n}\right)$. The number $i(M ; U \cdot V)$ is called the multiplicity of $M$ in the intersection of $U$ and $V$. 
It follows immediately from this definition that $i(M ; U \cdot V)=i(M ; V \cdot U)$.

3. Criterion for multiplicity one.

Lemma 1. Let $\mathrm{o}$ be a local ring, and let $x_{1}, \cdots, x_{n}$ be elements of $\mathrm{o}$ which generate an ideal which is primary for the ideal of nonunits. Let $K$ be a basic field of $\mathrm{D}$ and $m$ be the dimension of $\mathrm{o}$. Let $P\left(\cdots A_{i j} \cdots\right)$ be a polynomial not equal to 0 in $m n$ arguments $A_{i j}(1 \leqq i \leqq m, 1 \leqq j \leqq n)$ with coefficients in a field containing $K$. Then, it is possible to find $m n$ elements $a_{i j}$ of $K$ such that $P\left(\cdots a_{i j} \cdots\right) \neq 0$ and such that the elements $\sum_{j-1}^{n} a_{i j} x_{j}(1 \leqq i \leqq m)$ form $a$ system of parameters in a.

This is a slight improvement of the result of Proposition 7, L.R. §3, p. 703. The proof again proceeds by induction on $m$. Using the same notation as in L.R. (except that the numbers $r$ and $m$ are to be replaced by $m$ and $n$ ), we observe that we can find a polynomial $Q\left(A_{1,1}, \cdots, A_{1, n}\right) \neq 0$ in $n$ arguments with coefficients in $K$ such that, if $a_{1,1}, \cdots, a_{1, n}$ are any $n$ elements of $K$ such that $Q\left(a_{1,1}, \cdots, a_{1, n}\right) \neq 0$, then the element $y_{1}^{\prime}=\sum_{j=1}^{n} a_{1, j} x_{j}$ does not belong to any of the spaces $\mathfrak{M}_{i}(1 \leqq i \leqq g)$. Since $K$ contains infinitely many elements, we can find elements $a_{1,1}, \cdots, a_{1, n}$ in $K$ such that $P Q$ does not vanish identically by the substitution $A_{1, j} \rightarrow a_{1, j}(1 \leqq j \leqq n)$. Let $P^{*}$ be the result of this substitution; proceeding as in L.R., we can apply our induction assumption to the local ring $0 / 0 y_{1}$ and to the polynomial $P^{*}$.

LEMMA 2. Let $M$ be a variety of dimension $m$ in $E^{n}(X)$. We can find $m$ linear combinations $Y_{1}, \cdots, Y_{m}$ of $X_{1}, \cdots, X_{n}$ with coefficients in $K$ which have the following property: if $y_{1}, \cdots, y_{m}$ are the functions induced on $M$ by $Y_{1}, \cdots, Y_{m}$, then $\left\{y_{1}, \cdots, y_{m}\right\}$ is a system of parameters in $\mathrm{f}(M)$ and $\mathrm{P}(M)$ is separable over $K\left(\left(y_{1}, \cdots, y_{m}\right)\right)$.

Denote by $x_{1}, \cdots, x_{n}$ the functions induced by $X_{1}, \cdots, X_{n}$ on $M$; we have $\mathrm{f}(M)=K\left[\left[x_{1}, \cdots, x_{n}\right]\right]$. Assuming $K$ to be of characteristic $p \neq 0$, we have $\mathrm{P}(M)=(\mathrm{P}(M))^{p}\left(x_{1}, \cdots, x_{n}\right)$, which proves that $\left\{x_{1}, \cdots, x_{n}\right\}$ contains a $p$-base of $\mathrm{P}(M)\left({ }^{26}\right)$. Let $\left\{u_{1}, \cdots, u_{m}\right\}$ be any system of parameters in $\mathrm{f}(M)$; then $\mathrm{P}(M)$ is finite over $U=K\left(\left(u_{1}, \cdots, u_{m}\right)\right)$, and we have $U=U^{p}\left(u_{1}, \cdots, u_{m}\right),\left[U: U^{p}\right]=p^{m}$. We have $\left[\mathrm{P}(M): U^{p}\right]=[\mathrm{P}(M): U] \cdot\left[U: U^{p}\right]$ $=\left[\mathrm{P}(M):\left((\mathrm{P}(M))^{p}\right]\left[(\mathrm{P}(M))^{p}: U^{p}\right]\right.$, and also $[\mathrm{P}(M): U]=\left[\left((\mathrm{P}(M))^{p}: U^{p}\right]\right.$ because the mapping $x \rightarrow x^{p}$ is an isomorphism. It follows that $\left[\mathrm{P}(M):(\mathrm{P}(M))^{p}\right]$ $=\left[U: U^{p}\right]=p^{m}$. We may therefore assume without loss of generality that $x_{1}, \cdots, x_{m}$ form a $p$-base of $\mathrm{P}(M)$. Every monomial in $x_{1}, \cdots, x_{n}$ may be expressed as a linear combination with coefficients in $(\mathrm{P}(M))^{p}$ of the quantities $x_{1}^{e_{1}} \cdots x_{m}^{e_{m}}\left(0 \leqq e_{1}, \cdots, e_{m} \leqq p\right)$.

Introduce $m n$ letters $A_{i j}(1 \leqq i \leqq m, 1 \leqq j \leqq n)$ which are considered as algebraically independent over $\mathrm{P}(M)$, and set $y_{i}=\sum_{j=1}^{n} A_{i j} x_{j}(1 \leqq i \leqq m)$. Denote

${ }^{(26)}$ Cf. S. MacLane, Modular fields I. Separating transcendence bases, Duke Math. J. vol. 5 (1939) p. 372. 
by $\xi_{1}, \cdots, \xi_{p^{m}}$ the quantities $x_{1}^{e_{1}} \cdots x_{m}^{e_{m}}$ and by $\eta_{1}, \cdots, \eta_{p^{m}}$ the quantities $y_{1}^{e_{1}} \cdots y_{m}^{e_{m}}\left(0 \leqq e_{1}, \cdots, e_{m}<p\right)$. We have

$$
\eta_{k}=\sum_{l=1}^{p m} \phi_{k l}\left(\cdots A_{i j} \cdots\right) \xi_{l} \quad\left(1 \leqq k \leqq p^{m}\right)
$$

where each $\phi_{k l}$ is a polynomial with coefficients in $(\mathrm{P}(M))^{p}$. The determinant $P\left(\cdots A_{i j} \cdots\right)$ of the matrix $\left(\phi_{k l}\left(\cdots A_{i j} \cdots\right)\right)_{k, l}$ does not vanish identically, because it takes the value 1 when $A_{i j}=\delta_{i j}$. Making use of Lemma 1, we see that we can find elements $a_{i j}$ in $K$ such that $P\left(\cdots a_{i j} \cdots\right) \neq 0$ and such that the elements $y_{i}=\sum_{j=1}^{n} a_{i j} x_{j}$ form a system of parameters in $f(M)$. The $p^{m}$ elements $y_{1}^{e_{1}} \ldots y_{m}^{e_{m}}$ are linearly independent with respect to $(\mathrm{P}(M))^{p}$, whence $\mathrm{P}(M)=(\mathrm{P}(M))^{p}\left(y_{1}, \cdots, y_{m}\right)$. If $Y$ is the field $K\left(\left(y_{1}, \cdots, y_{m}\right)\right)$, we know that $\mathrm{P}(M)$ is finite over $Y$ and that $\mathrm{P}(M)=Y(\mathrm{P}(M))^{p}$. It is well known that these conditions imply that $\mathrm{P}(M)$ is separable over $Y$. Lemma 2 is thereby proved.

Proposition 1. Let $M$ be a variety of dimension $m$ in $E^{n}(X)$. Then the neighborhood ring $\mathfrak{N}(M)$ is a regular local ring of dimension $n-m$. The completion $\overline{\mathfrak{N}}(M)$ contains a field $Z$ isomorphic with $\mathrm{P}(M)$ which is a complete system of representatives for the residue classes modulo its ideal of nonunits; $\overline{\mathfrak{N}}(M)$ is isomorphic to a ring of power series with coefficients in $Z$. If $Y_{1}, \cdots, Y_{m}$ are $m$ linear combinations of $X_{1}, \cdots, X_{n}$ with the property described in Lemma 2, the field $Z$ may be taken to contain $K\left(\left(Y_{1}, \cdots, Y_{m}\right)\right)$ and is then uniquely determined.

We determine $m$ linear combinations $Y_{1}, \cdots, Y_{m}$ of $X_{1}, \cdots, X_{n}$ which have the property described in Lemma 2, and we adjoin to these $n-m$ other linear combinations $Y_{m+1}, \cdots, Y_{n}$ in such a way that $Y_{1}, \cdots, Y_{n}$ are linearly independent. It is clear that no power series not equal to 0 in $Y_{1}, \cdots, Y_{m}$ vanishes on $M$, whence $K\left(\left(Y_{1}, \cdots, Y_{m}\right)\right) \subset \overline{\mathfrak{N}}(M)$. The field $K\left(\left(Y_{1}, \cdots, Y_{m}\right)\right)$ is a basic field of $\mathfrak{N}(M)$; it follows from Proposition 3, L.R., $\S 3$, p. 701 , that $K\left(\left(Y_{1}, \cdots, Y_{m}\right)\right)$ is contained in a subfield $Z$ of $\overline{\mathfrak{R}}(M)$ which is a complete system of representatives for the residue classes modulo the ideal of nonunits. It is clear that every element of $\overline{\mathfrak{N}}(M)$ which is algebraic over $K\left(\left(Y_{1}, \cdots, Y_{m}\right)\right)$ is contained in $Z$, which proves that $Z$ is uniquely determined. Let $\eta_{1}, \cdots, \eta_{n}$ be the elements of $Z$ which belong to the residue classes of $Y_{1}, \cdots, Y_{n}$; we have $\eta_{j}=Y_{j}$ for $1 \leqq j \leqq m$. Every power series $F\left(X_{1}, \cdots, X_{n}\right)$ with coefficients in $K$ may be expressed in the form of a power series $F^{*}\left(Y_{1}, \cdots, Y_{n}\right)$ in $Y_{1}, \cdots, Y_{n}$; it is clear that, if $F$ vanishes on $M$, we have $F^{*}\left(\eta_{1}, \cdots, \eta_{n}\right)=0\left({ }^{27}\right)$. Remembering that $Y_{j}=\eta_{j}$

$\left.{ }^{27}\right)$ The ring $K\left[\left[\eta_{1}, \cdots, \eta_{m}\right]\right]\left[\eta_{m+1}, \cdots, \eta_{n}\right]$ is isomorphic with a subring of $f(M)$ and is integral over $K\left[\left[\eta_{1}, \cdots, \eta_{m}\right]\right]$. By Proposition 3, L.R., $\S I I$, p. 694, it is a complete ring; it follows immediately that it is isomorphic with $f(M)$. Since $\eta_{1}, \cdots, \eta_{n}$ are clearly nonunits in this ring, the expression $F^{*}\left(\eta_{1}, \cdots, \eta_{n}\right)$ has a meaning. 
for $j \leqq m$, we see that any power series which vanishes on $M$ belongs to the ideal generated in $\overline{\mathfrak{R}}(M)$ by the $n-m$ elements $Y_{m+1}-\eta_{m+1}, \cdots, Y_{n}-\eta_{n}$. Let $\mathfrak{V}$ be this ideal; then $\mathfrak{V}$ is the ideal of nonunits in $\overline{\mathfrak{R}}(M)$. Since we know already that $\mathfrak{N}(M)$ is a local ring of dimension $n-m$, it follows that $\overline{\mathfrak{N}}(M)$ is a regular ring.

Since $\mathfrak{N}(M)$ is dense in $\overline{\mathfrak{N}}(M)$, we can find $n-m$ elements $G_{m+1}, \cdots, G_{n}$ in $\mathfrak{N}(M)$ such that $G_{j} \equiv Y_{j}-\eta_{j}\left(\bmod \mathfrak{Y}^{2}\right)(1 \leqq j \leqq n)$. If $\mathfrak{Y}^{\prime}$ is the ideal generated by $G_{m+1}, \cdots, G_{n}$ in $\overline{\mathfrak{N}}(M)$, we have $\mathfrak{Y} \subset \mathfrak{Y}^{\prime}+\mathfrak{Y}^{2}$, whence, by induction, $\mathfrak{Y C} \mathfrak{Y}^{\prime}+\mathfrak{Y}^{k}$ for every $k$. Making use of Lemma 6, L.R., §2, p. 695, we conclude that $\mathfrak{Y}=\mathfrak{Y}^{\prime}$; it follows that $G_{m+1}, \cdots, G_{n}$ generate the ideal of nonunits in $\mathfrak{N}(M)$ and that $\mathfrak{N}(M)$ is a regular local ring.

Proposition 2. Let $M$ be a variety of dimension $m$ in $E^{n}(X)$, and let $F_{1}, \cdots, F_{n-m}$ be $n-m$ power series which vanish on $M$. The following assertions are equivalent: (1) $F_{1}, \cdots, F_{n-m}$ form a regular system of parameters in $\mathfrak{N}(M)$; (2) the Jacobian matrix of $F_{1}, \cdots, F_{n-m}$ is of rank $n-m$ on $M$.

(The Jacobian matrix of several power series is the matrix formed by the partial derivatives of these power series with respect to their arguments; when we say that this Jacobian matrix is of rank $n-m$ on $M$, we mean that the functions induced on $M$ by the coefficients of the matrix form a matrix of rank $n-m$.)

We use the notation of the proof of Proposition 1 . If $F$ is any power series in $X_{1}, \cdots, X_{n}$, we set $F^{*}\left(Y_{1}, \cdots, Y_{n}\right)=F\left(X_{1}, \cdots, X_{n}\right)$ and $F^{* *}\left(Z_{m+1}, \cdots, Z_{n}\right)=F^{*}\left(\eta_{1}, \cdots, \eta_{m}, Z_{m+1}+\eta_{m+1}, \cdots, Z_{n}+\eta_{n}\right) ; F^{* *}$ is therefore a power series with coefficients in the field $Z$. Assume that (1) holds; then the $n-m$ power series $F_{i}^{* *}\left(Z_{m+1}, \cdots, Z_{n}\right)(1 \leqq i \leqq n-m)$ generate the ideal of nonunits in $Z\left[\left[Z_{m+1}, \cdots, Z_{n}\right]\right]$ and $Z_{m+1}, \cdots, Z_{n}$ belong to $Z\left[\left[F_{1}^{* *}, \cdots, F_{n-m}^{* *}\right]\right]$. It follows immediately that the functional determinant of $F_{1}^{* *}, \cdots, F_{n-m}^{* *}$ is a unit in $\overline{\mathfrak{N}}(M)$, or that the functional determinant of $F_{1}^{*}, \cdots, F_{n-m}^{*}$ with respect to $Y_{m+1}, \cdots, Y_{n}$ does not vanish on $M$. This implies of course that the Jacobian matrix of $F_{1}, \cdots, F_{n-m}$ is of rank $n-m$ on $M$.

Conversely, assume that the Jacobian matrix of $F_{1}, \cdots, F_{n-m}$ is of rank $n-m$ on $M$. Let $G_{1}, \cdots, G_{n-m}$ be $n-m$ power series which form a regular system of parameters in $\mathfrak{N}(M)$. Then we can write $F_{i}=\sum_{j=1}^{n-m} A_{i j} G_{j}$ $(1 \leqq i \leqq n-m)$ with $A_{i j} \in \mathfrak{N}(M)$. Let $D$ be a power series which does not vanish on $M$ and which is such that $D A_{i j} \in K[[X]](1 \leqq i, j \leqq n-m)$. If $m$ is the prime ideal which corresponds to $M$, we have

$$
D \partial F_{i} / \partial X_{k} \equiv \sum_{j=1}^{n-m}\left(D A_{i j}\right) \partial G_{j} / \partial X_{k}(\bmod \mathfrak{m}) .
$$

It follows that the determinant of the matrix $\left(A_{i j}\right)$ does not vanish on $M$ and therefore that $G_{1}, \cdots, G_{n-m}$ belong to the ideal generated by $F_{1}, \cdots, F_{n-m}$ 
in $\mathfrak{N}(M)$. This means that $F_{1}, \cdots, F_{n-m}$ form a regular system of parameters in $\mathfrak{R}(M)$.

Remark. If $F$ is any other power series which vanishes on $M, F$ may be expressed as a linear combination of $F_{1}, \cdots, F_{n-m}$ with coefficients in $\mathfrak{N}(M)$. We see immediately that the partial derivatives of an element of $\mathfrak{N}(M)$ belong themselves to $\mathfrak{N}(M)$. It follows easily that, if $F_{1}^{\prime}, \cdots, F_{a}^{\prime}$ are any number of power series which vanish on $M$, their Jacobian matrix is of rank not greater than $n-m$ on $M$.

Definition 1. Let $M$ be a subvariety of $U$ in $E^{n}(X)$. If the local ring $\mathfrak{N}_{U}(M)$ is regular, we say that $M$ is simple on $U$. If not, we say that $M$ is singular on $U$.

Proposition 3. Let $M$ be a subvariety of a variety $U$ in $E^{n}(X)$. A necessary and sufficient condition for $M$ to be simple on $U$ is that there should exist a regular system of parameters in $\mathfrak{R}(M)$ which contains as a subset a system of parameters in $\mathfrak{N}(U)$.

Let $\mathfrak{u}$ be the prime ideal which corresponds to $U$. Then $\mathfrak{R}_{U}(M)$ is isomorphic to the ring $\mathfrak{N}(M) / \mathfrak{u} \mathfrak{N}(M)$ and $\mathfrak{N}(U)$ is the ring of quotients of $\mathfrak{u} \mathfrak{N}(M)$ with respect to $\mathfrak{R}(M)$. This being said, Proposition 3 follows immediately from Proposition 9, L.R., §III, p. 705.

Remark. If a system of parameters in $\mathfrak{N}(U)$ can be extended to a regular system of parameters in $\mathfrak{R}(M)$, the elements of this system of parameters in particular must belong to $\mathfrak{N}(M)$. Multiplying them by units in $\mathfrak{N}(M)$, we see that these elements may be assumed to belong to $K[[X]]$.

Proposition 4. Let $M$ be a proper component of the intersection of two algebroid varieties $U$ and $V$ in $E^{n}(X)$. Assume that $M$ is simple on $V$ and let $G_{1}, \cdots, G_{n \rightarrow v}$ be elements of $K[[X]]$ which form a system of parameters in $\mathfrak{N}(V)$ and which can be included in a regular system of parameters in $\mathfrak{R}(M)$. Let $G_{i}^{U}$ be the function induced on $U$ by $G_{i}(1 \leqq i \leqq n-v)$; then the $n-v$ functions $G_{i}^{U}$ form a system of parameters in $\mathfrak{N}_{U}(M)$ and we have $i(M ; U \cdot V)=e\left(\mathfrak{N}_{U}(M)\right.$; $\left.G_{1}^{U}, \cdots, G_{n-v}^{U}\right)$.

We use the notation of Definition $3, \S 2$, p. 31 . Let $\mathfrak{u}$ be the prime ideal which corresponds to $U$ in $K[[X]]$, and let $\mathfrak{u}_{1}$ be the ideal generated by $\mathfrak{u}$ in $K\left[\left[X, X^{\prime}\right]\right] ; \mathfrak{u}_{1}$ is the prime ideal which corresponds to the variety $U \times E^{n}\left(X^{\prime}\right)$. Let $\xi_{k}$ and $\xi_{k}^{\prime}$ be the functions induced by $X_{k}$ and $X_{k}^{\prime}$ on $U \times E^{n}\left(X^{\prime}\right)$, and let $\Gamma_{1}, \cdots, \Gamma_{n-v}$ be the functions induced by $G_{1}\left(X_{1}^{\prime}, \cdots, X_{n}^{\prime}\right), \cdots$, $G_{n-v}\left(X_{1}^{\prime}, \cdots, X_{n}^{\prime}\right)$ on $U \times E^{n}\left(X^{\prime}\right)$. The ideal generated by $G_{1}, \cdots, G_{n-v}$ in $\mathfrak{R}(M)$ being by assumption prime, every function which vanishes on $V$ may be written as a linear combination of $G_{1}, \cdots, G_{n-v}$ with coefficients in $\mathfrak{R}(M)$. It follows immediately that the ideal generated by $\Gamma_{1}, \cdots, \Gamma_{n-v}$ in $\mathfrak{N}_{U \times E^{n}\left(X^{\prime}\right)}\left(M^{\Delta}\right)$ is prime and that the corresponding factor ring of $\mathfrak{N}_{U \times E^{n}\left(X^{\prime}\right)}\left(M^{\Delta}\right)$ is $\mathfrak{N}_{U \times V^{\prime}}\left(M^{\Delta}\right)$. Making use of the corollary to Theorem 5, $\S 5$, Part I, p. 25 , we obtain 


$$
\begin{aligned}
i(M ; U \cdot V) & =e\left(\mathfrak{N}_{U \times V^{\prime}}\left(M^{\Delta}\right) ; x_{1}^{\prime}-x_{1}, \cdots, x_{n}{ }^{\prime}-x_{n}\right) \\
& =e\left(\Re_{U \times E^{n}\left(X^{\prime}\right)}\left(M^{\Delta}\right) ; \xi_{1}^{\prime}-\xi_{1}, \cdots, \xi_{n}{ }^{\prime}-\xi_{n} ; \Gamma_{1}, \cdots, \Gamma_{n-v}\right) .
\end{aligned}
$$

The ideal generated by $\mathfrak{u}$ and $X_{1}^{\prime}-X_{1}, \cdots, X_{n}^{\prime}-X_{n}$ in $K\left[\left[X, X^{\prime}\right]\right]$ is the prime ideal which corresponds to $U^{\Delta}$. It follows that the ideal generated by $\xi_{1}^{\prime}-\xi_{1}, \cdots, \xi_{n}^{\prime}-\xi_{n}$ in $\mathfrak{R}_{U \times E^{n}\left(X^{\prime}\right)}\left(M^{\Delta}\right)$ is prime and that the corresponding factor ring is the ring $\mathfrak{N}_{U} \Delta\left(M^{\Delta}\right)$. Let $\Gamma_{1}^{*}, \cdots, \Gamma_{n \rightarrow v}^{*}$ be the functions induced by $G_{1}, \cdots, G_{n \rightarrow v}$ on $U^{\Delta}$; then $\Gamma_{i}^{*}$ is also the function induced by $\Gamma_{i}$ on $U^{\Delta}$ because $X_{k}$ and $X_{k}^{\prime}$ induce the same function on $U^{\Delta}$. Making use again of the result quoted a few lines above, we obtain

$$
i(M ; U \cdot V)=e\left(\Re_{U \Delta}\left(M^{\Delta}\right) ; \Gamma_{1}^{*}, \cdots, \Gamma_{n-v}^{*}\right) .
$$

Taking into account the natural isomorphism which exists between $f\left(U^{\Delta}\right)$ and $f(U)$, we see that $\mathfrak{R}_{M} \Delta\left(U^{\Delta}\right)$ is isomorphic with $\mathfrak{N}_{M}(U)$ under an isomorphism which maps $\Gamma_{i}^{*}$ upon $G_{i}^{U}(1 \leqq i \leqq n-v)$. Proposition 4 is thereby proved.

Proposition 5. Let $M$ be a proper component of the intersection of two algebroid varieties $U$ and $V$. Using the notation of Definition $3, \S 2$, p. 31 , we have $i(M ; U \cdot V)=i\left(M^{\Delta} ;\left(U \times V^{\prime}\right) \cdot \Delta\right)$.

Let us select $n-m$ elements $H_{1}, \cdots, H_{n-m}$ of $K[[X]]$ which form a regular system of parameters in $\mathfrak{R}(M)$. Then every element of the ideal $\mathfrak{m}$ which corresponds to $M$ is a linear combination of $H_{1}, \cdots, H_{n-m}$ with coefficients in $\mathfrak{R}(M)$. The ideal generated by $\mathfrak{m}$ and $X_{1}^{\prime}-X_{1}, \cdots, X_{n}^{\prime}-X_{n}$ in $K\left[\left[X, X^{\prime}\right]\right]$ is the prime ideal which corresponds to $M^{\Delta}$. It follows immediately that the maximal prime ideal of $\mathfrak{N}\left(M^{\Delta}\right)$ is generated by $H_{1}, \cdots, H_{n-m}$, $X_{1}^{\prime}-X_{1}, \cdots, X_{n}^{\prime}-X_{n}$; that is, these elements form a regular system of parameters in $\mathfrak{R}\left(M^{\Delta}\right)$. It follows from Proposition 4 that $i\left(M^{\Delta} ;\left(U \times V^{\prime}\right) \cdot \Delta\right)$ $=e\left(\Re_{U \times V^{\prime}}\left(M^{\Delta}\right) ; x_{1}^{\prime}-x_{1}, \cdots, x_{n}^{\prime}-x_{n}\right)=i(M ; U \cdot V)$.

Proposition 5 allows us to reduce problems of multiplicities of intersections of arbitrary varieties to similar problems in which one of the varieties to be intersected is linear.

Proposition 6. Let $M$ be a proper component of the intersection of two algebroid varieties $U$ and $V$ in $E^{n}(X)$, and let $\mathfrak{u}$ and $\mathfrak{v}$ be the prime ideals in $K[[X]]$ which correspond to $U$ and $V$. Then the following two assertions are equivalent: (1) $i(M ; U \cdot V)=1$; (2) the ideal generated by $\mathfrak{u}$ and $\mathfrak{v}$ in $\mathfrak{N}(M)$ is prime.

Assume first that (1) holds. Then $\mathfrak{N}_{U \times V^{\prime}}\left(M^{\Delta}\right)$ is a regular local ring (by Theorem 3, §2, Part I, p. 14).

Let $m, u$ and $v$ be the dimensions of $M, U$ and $V$ respectively (whence $m=u+v-n)$. By Proposition 9, L.R., §III, p. 705, we can find $2 n-u-v$ elements $H_{1}, \cdots, H_{2 n-u-v}$ of $K\left[\left[X, X^{\prime}\right]\right]$ which form a set of generators of the prime ideal which corresponds to $U \times V^{\prime}$ in $\mathfrak{N}\left(M^{\Delta}\right)$, and which are such that $X_{1}^{\prime}-X_{1}, \cdots, X_{n}^{\prime}-X_{n}, H_{1}, \cdots, H_{2 n-u-v}$ form a regular system of 
parameters in $\mathfrak{N}\left(M^{\Delta}\right)$. The elements $H_{1}, \cdots, H_{2 n-u-v}$ belong to the ideal generated in $K\left[\left[X, X^{\prime}\right]\right]$ by $\mathfrak{u}$ and by the prime ideal $\mathfrak{v}^{\prime}$ which corresponds to $V^{\prime}$ in $K\left[\left[X^{\prime}\right]\right]$. If $F$ is a power series in the letters $X$ alone which vanishes on $M, F$ may be expressed as a linear combination of $X_{1}^{\prime}-X_{1}, \cdots, X_{n}^{\prime}-X_{n}$, $H_{1}, \cdots, H_{2 n-u-v}$ with coefficients in $\mathfrak{N}\left(M^{\Delta}\right)$. If we make the substitution $X_{1}^{\prime} \rightarrow X_{1}, \cdots, X_{n}^{\prime} \rightarrow X_{n}$ in a power series which does not vanish on $M^{\Delta}$, we obtain a power series which does not vanish on $M$; it follows immediately that $F$ is a linear combination of $H_{1}(X, X), \cdots, H_{2 n-u-v}(X, X)$ with coeffcients in $\mathfrak{R}(M)$. Since $H_{i}\left(X, X^{\prime}\right)$ belongs to the ideal generated by $\mathfrak{u}$ and $\mathfrak{v}^{\prime}$, this proves that the ideal generated by $\mathfrak{u}$ and $\mathfrak{b}$ in $\mathfrak{R}(M)$ is the ideal of nonunits in this ring.

Assume now that (2) holds. Then we can find $n-m=2 n-u-v$ elements $H_{1}, \cdots, H_{2 n-u-v}$ of the ideal generated by $\mathfrak{u}$ and $\mathfrak{v}$ which generate the ideal of nonunits in $\mathfrak{N}(M)$ (observe that, $M$ being a component of the intersection of $U$ and $V$, the only prime ideal in $\mathfrak{N}(M)$ which contains $\mathfrak{u}$ and $\mathfrak{v}$ is the ideal of nonunits). Every function of the ideal $m$ which corresponds to $M$ is a linear combination of $H_{1}, \cdots, H_{2 n-u-v}$ with coefficients in $\Re(M)$. The ideal which corresponds to $M^{\Delta}$ is the ideal generated by $m$ and $X_{1}^{\prime}-X_{1}, \cdots, X_{n}^{\prime}-X_{n}$; it follows immediately that $X_{1}^{\prime}-X_{1}, \cdots, X_{n}^{\prime}-X_{n}, H_{1}, \cdots, H_{2 n-u-v}$ form a regular system of parameters in $\mathfrak{N}\left(M^{\Delta}\right)$. The equality $i(M ; U \cdot V)=1$ follows from this fact by making use of Proposition 9, L.R., §III, p. 705, and of the corollary to Theorem 5, $\$ 5$, part I, p. 25.

DEFINITION 2. Let $M$ be a component of the intersection of two algebroid varieties $U$ and $V$ in $E^{n}(X)$, of respective dimensions $u$ and $v$. We shall say that $U$ and $V$ are in general position with respect to each other along $M$ when it is possible to find $n-u$ power series $F_{1}, \cdots, F_{n-u}$ which vanish on $U$ and $n-v$ power series $G_{1}, \cdots, G_{n-v}$ which vanish on $V$ such that the Jacobian matrix of $F_{1}, \cdots, F_{n-u}, G_{1}, \cdots, G_{n-v}$ is of rank $2 n-u-v$ on $M$.

THEOREM 2. Let $M$ be a component of the intersection of two algebroid varieties $U$ and $V$ in $E^{n}(X)$. A necessary and sufficient condition for $M$ to be $a$ proper component of multiplicity 1 in this intersection is for $U$ and $V$ to be in general position with respect to each other along $M$.

Assume that $U$ and $V$ are in general position with respect to each other along $M$. It follows from the remark which follows the proof of Proposition 2 that $M$ is of dimension not greater than $n-(2 n-u-v)=u+v-n$. Making use of Theorem $1, \S 2$, p. 31 we see that $M$ is a proper component of the intersection of $U$ and $V$. Proposition 2 then shows that (using the notation introduced in Definition 2) $F_{1}, \cdots, F_{n-u}, G_{1}, \cdots, G_{n-v}$ form a regular system of parameters in $\mathfrak{N}(M)$. It follows that the ideal generated in $\mathfrak{N}(M)$ by the prime ideals $\mathfrak{u}$ and $\mathfrak{b}$ which correspond to $U$ and $V$ is prime. The equality $i(M ; U \cdot V)=1$ then follows by Proposition 6 .

Assume now that $M$ is a proper component of multiplicity 1 of the inter- 
section of $U$ and $V$. Then, by Propositon 6, there exists a regular system of parameters $\left\{H_{1}, \cdots, H_{2 n-u-v}\right\}$ in $\mathfrak{R}(M)$ whose elements belong to the ideal generated by $\mathfrak{u}$ and $\mathfrak{v}$ in $K[[X]]$. We can find a finite number of elements $F_{1}, \cdots, F_{a}, G_{1}, \cdots, G_{b}$ such that $F_{k} \in \mathfrak{u}, G_{l} \in \mathfrak{v} .(1 \leqq k \leqq a, 1 \leqq l \leqq b)$ and such that each $H_{i}(1 \leqq i \leqq 2 n-u-v)$ is a linear combination of $F_{1}, \cdots, F_{a}$, $G_{1}, \cdots, G_{b}$ with coefficients in $K[[X]]$. It follows that the Jacobian matrix of the $a+b$ power series $F_{1}, \cdots, F_{a}, G_{1}, \cdots, G_{b}$ is of rank $2 n-u-v$ on $M$; we may assume that the Jacobian matrix of $F_{1}, \cdots, F_{a^{\prime}}, G_{1}, \cdots, G_{b^{\prime}}$ is of rank $2 n-u-v$ on $M$ where $a^{\prime}$ and $b^{\prime}$ are indices such that $a^{\prime}+b^{\prime}=2 n-u-v$. The Jacobian matrix of $F_{1}, \cdots, F_{a^{\prime}}$ is of rank not greater than $n-u$ on $U$ and, a fortiori, on $M$. The Jacobian matrix of $G_{1}, \cdots, G_{b}$, is of rank not greater than $n-v$ on $V$ and, a fortiori, on $M$. It follows immediately that we must have $a^{\prime}=n-u, b^{\prime}=n-v$, which proves that $U$ and $V$ are in general position with respect to each other along $M$.

COROLLARY. If $M$ is a proper component of multiplicity 1 of the intersection of $U$ and $V$, then $M$ is simple on both $U$ and $V$.

In fact, using the notation of Definition 2, the elements $F_{1}, \cdots, F_{n-u}$, $G_{1}, \cdots, G_{n-v}$ form a regular system of parameters in $\mathfrak{N}(M)$. The ideal generated by $G_{1}, \cdots, G_{n-v}$ in $\mathfrak{R}(M)$ is prime of dimension $n-u$ and is contained in the ideal generated by $\mathfrak{b}$ (where $\mathfrak{b}$ is the ideal which corresponds to $V$ in $K[[X]])$; furthermore, the factor ring of $\mathfrak{R}(M)$ by this ideal is regular. Since the ideal $\mathfrak{v} \mathfrak{N}(M)$ is also of dimension $n-u$ in $\mathfrak{R}(M)$, we conclude that $\mathfrak{N}_{V}(M)=\mathfrak{N}(M) / \mathfrak{b} \mathfrak{N}(M)$ is regular, which proves that $M$ is simple on $V$. We would see in the same way that $M$ is simple on $U$.

4. Intersections of product varieties. We consider two series of letters $\left\{X_{1}, \cdots, X_{m}\right\}$ and $\left\{Y_{1}, \cdots, Y_{n}\right\}$.

Theorem 3. Let $U$ and $V$ be algebroid varieties in $E^{m}(X)$; let $S$ and $T$ be algebroid varieties in $E^{n}(Y)$. Denote by $M_{1}, \cdots, M_{a}$ the components of the intersection of $U$ and $V$ and by $N_{1}, \cdots, N_{b}$ the components of the intersection of $S$ and $T$. Then the components of the intersection of $U \times S$ and $V \times T$ in $E^{m}(X) \times E^{n}(Y)$ are the varieties $M_{i} \times N_{j}(1 \leqq i \leqq a, 1 \leqq j \leqq b)$.

Let $P$ be a component of the intersection of $U \times S$ and $V \times T$. Since $P \subset U \times S$, we have $\operatorname{pr}_{x} P \subset \operatorname{pr}_{x}(U \times S)=U$; we see in the same way that $\operatorname{pr}_{X} P \subset V, \operatorname{pr}_{Y} P \subset S, \operatorname{pr}_{Y} P \subset T$. It follows that $\operatorname{pr}_{X} P$ is contained in some component of the intersection of $U$ and $V$, say in $M_{i}$, and that $\operatorname{pr}_{Y} P$ is contained in some component, say $N_{j}$, of the intersection of $S$ and $T$. We have $P \subset\left(\operatorname{pr}_{X} P\right) \times\left(\operatorname{pr}_{Y} P\right) \subset M_{i} \times N_{j}$. But $M_{i} \times N_{j}$ is clearly contained in both $U \times S$ and $V \times T$; it follows that $P=M_{i} \times N_{j}$.

Let now $k$ and $l$ be any two indices such that $1 \leqq k \leqq a, 1 \leqq l \leqq b$. Then $M_{k} \times N_{l}$ is contained in some component $P$ of the intersection of $U \times S$ and $V \times T$, and we have seen that $P$ is of the form $M_{i} \times N_{j}$. It follows that 
$M_{k} \subset M_{i}, N_{l} \subset N_{j}$, whence $M_{k}=M_{i}, N_{l}=N_{j}$. This proves that $M_{k} \times N_{l}$ is a component of the intersection of $U \times S$ and $V \times T$. Theorem 3 is thereby proved.

THEOREM 4. Let $U$ and $V$ be algebroid varieties in $E^{m}(X)$; let $S$ and $T$ be algebroid varieties in $E^{n}(Y)$. Let $M$ be a component of the intersection of $U$ and $V$ and let $N$ be a component of the intersection of $S$ and $T$. Then $M \times N$ is a proper component of the intersection of $U \times S$ and $V \times T$ if and only if $M$ and $N$ are proper components of their respective intersections. If this condition is satisfied, we have

$$
i(M \times N ;(U \times S) \cdot(V \times T))=i(M ; U \cdot V) \cdot i(N ; S \cdot T) .
$$

Let $u, v, s, t, \mu, \nu$ be the respective dimensions of $U, V, S, T, M, N$. Then we have $m+n-(u+s)-(v+t)+(\mu+\nu)=(m-u-v+\mu)+(n-s-t+\nu)$. The left side of this formula and both terms on the right side are always greater than or equal to 0 ; if the left side is 0 , both terms on the right side must be 0 , and conversely. This proves the first assertion of Theorem 4.

Let us construct copies $E^{m}\left(X^{\prime}\right)$ and $E^{n}\left(Y^{\prime}\right)$ of the spaces $E^{m}(X)$ and $E^{n}(Y)$. We denote by $V^{\prime}$ and $T^{\prime}$ the copies of $V$ and $T$ in $E^{m}\left(X^{\prime}\right)$ and $E^{n}\left(Y^{\prime}\right)$ respectively. Let $\Delta_{X}$ and $\Delta_{Y}$ be the diagonals of the spaces $E^{m}(X) \times E^{m}\left(X^{\prime}\right)$ and $E^{n}(Y) \times E^{n}\left(Y^{\prime}\right)$. We may consider $E^{m+n}\left(X^{\prime}, Y^{\prime}\right)$ as a copy of $E^{m+n}(X, Y)$; we denote by $\Delta_{X, Y}$ the diagonal of $E^{m+n}(X, Y) \times E^{m+n}\left(X^{\prime}, Y^{\prime}\right)$. We have (Proposition 5, §3, p. 36)

$$
\begin{aligned}
i(M \times N ;(U \times S) \cdot(V \times T)) & =i\left((M \times N)^{\Delta_{X, Y}} ;\left((U \times S) \times\left(V^{\prime} \times T^{\prime}\right)\right) \cdot \Delta_{X, Y}\right), \\
i(M ; U \cdot V) & =i\left(M^{\Delta_{X}} ;\left(U \times V^{\prime}\right) \cdot \Delta_{X}\right), \\
i(N ; S \cdot T) & =i\left(N^{\Delta_{Y}} ;\left(S \times T^{\prime}\right) \cdot \Delta_{Y}\right) .
\end{aligned}
$$

It is clear that $\Delta_{X, Y} \stackrel{\bullet}{=} \Delta_{X} \times \Delta_{Y}$ and that $(M \times N)^{\Delta_{X}, Y}=M^{\Delta_{X}} \times N^{\Delta_{Y}}$; also we have $(U \times S) \times\left(V^{\prime} \times T^{\prime}\right)=\left(U \times V^{\prime}\right) \times\left(S \times T^{\prime}\right)$. It follows that we can reduce the general case to the case where $V$ and $T$ are linear varieties (the roles of $U, V, S, T, M, N$ being now played by $\left.U \times V^{\prime}, \Delta_{X}, S \times T^{\prime}, \Delta_{Y}, M^{\Delta x}, N^{\Delta_{Y}}\right)$. A fortiori, we may assume without loss of generality that $M$ is simple on $V$ and that $N$ is simple on $T$.

We can find $m$ linearly independent linear combinations $X_{1}^{*}, \cdots, X_{m}{ }^{*}$ of $X_{1}, \cdots, X_{m}$ with coefficients in $K$ which are such that $\mathrm{P}(M)$ is algebraic and separable over the field of quotients of $K\left[\left[x_{1}^{*}, \cdots, x_{\mu}^{*}\right]\right]$ (where $x_{i}^{*}$ is the function induced by $X_{i}^{*}$ on $M$ ). Let $Y_{1}^{*}, \cdots, Y_{n}^{*}$ be linear combinations of $Y_{1}, \cdots, Y_{n}$ with coefficients in $K$ which enjoy a similar property with respect to $N$. The ring $K[[X, Y]]$ is clearly a Kroneckerian product of $K[[X]]$ and $K[[Y]]$ over $K$; it follows from Lemma 3 , 33 , part I, p. 18 that $\mathfrak{f}(M \times N)$ is a Kroneckerian product of $\mathfrak{f}(M)$ and $\mathfrak{f}(N)$ over $K$. It follows from Lemma $4, \S 3$, part I, p. 19 that $x_{1}^{*}, \cdots, x_{\mu}^{*}, y_{1}^{*}, \cdots, y_{v}{ }^{*}$ are analytically independent over $K$ in $\mathrm{f}(M \times N)$ and that $\mathrm{P}(M \times N)$ is generated by 
adjunction to $K\left(\left(x_{1}^{*}, \cdots, x_{\mu}^{*}, y_{1}^{*}, \cdots, y_{\nu}^{*}\right)\right)$ of the elements of $\mathrm{P}(M)$ and $\mathrm{P}(N)$. It follows that $\mathrm{P}(M \times N)$ is algebraic and separable over $K\left(\left(x_{1}^{*}, \cdots, x_{\mu}^{*}, y_{1}^{*}, \cdots, y_{v}^{*}\right)\right)$. This being the case, we have seen in $\S 3$ that $\overline{\mathfrak{N}}(M+N)$ contains a field $\Lambda$ which is a complete system of representatives for the residue classes modulo the ideal of nonunits and which contains $K\left(\left(X_{1}^{*}, \cdots, X_{\mu}^{*}, Y_{1}^{*}, \cdots, Y_{\nu}^{*}\right)\right)$. Moreover, if $\xi_{k}$ and $\eta_{l}$ are the elements of $\Lambda$ which represent the residue classes of $X_{k}^{*}$ and $Y_{l}^{*}(\mu+1 \leqq k \leqq m$, $\nu+1 \leqq l \leqq n), \overline{\mathfrak{R}}(M \times N)$ is the ring of power series $\Lambda\left[\left[X_{\mu+1}^{*}-\xi_{\mu+1}, \cdots\right.\right.$, $\left.\left.X_{m}^{*}-\xi_{m}, Y_{\nu+1}^{*}-\eta_{v+1}, \cdots, Y_{n}^{*}-\eta_{n}\right]\right]$. Let $\Lambda_{M}$ be the field obtained by adjunction of $\xi_{\mu+1}, \cdots, \xi_{m}$ to $K\left(\left(X_{1}^{*}, \cdots, X_{\mu}^{*}\right)\right)$ and let $\Lambda_{N}$ be the field obtained by adjunction of $\eta_{v+1}, \cdots, \eta_{n}$ to $K\left(\left(Y_{1}^{*}, \cdots, Y_{v}^{*}\right)\right)$. If we represent an element of $K[[X]]$ as a power series with coefficients in $\Lambda$ in the elements $X_{k}^{*}-\xi_{k}, Y_{l}^{*}-\eta_{l}(\mu+1 \leqq k \leqq m, \nu+1 \leqq l \leqq n)$, it is clear $\left.{ }^{28}\right)$ that the expression will contain only the $X_{k}^{*}-\xi_{k}$ 's, and that the coefficients will belong to $\Lambda_{M}$. It follows immediately that the ring $\Lambda_{M}\left[\left[X_{\mu+1}^{*}-\xi_{\mu+1}, \cdots, X_{m}^{*}-\xi_{m}\right]\right]$ contains $\mathfrak{N}(M)$ and is a completion $\mathfrak{\Re}(M)$ of $\mathfrak{N}(M)$. In the same way, we see that the ring $\Lambda_{N}\left[\left[Y_{v+1}^{*}-\eta_{v+1}, \cdots, Y_{n}^{*}-\eta_{n}\right]\right]$ is a completion $\bar{\Re}(N)$ of $\mathfrak{N}(N)$. We may therefore consider $\overline{\mathfrak{N}}(M \times N)$ as a Kroneckerian product of $\overline{\mathfrak{R}}(M)$ and $\overline{\mathfrak{R}}(N)$ over $\Lambda$.

Since $M$ is regular on $V$, we can find $m-v$ elements $F_{1}, \cdots, F_{m-v}$ of $K[[X]]$ which form a system of parameters in $\mathfrak{N}(V)$ and which can be included in a regular system of parameters $\left\{F_{1}, \cdots, F_{m-\mu}\right\}$ in $\mathfrak{N}(M)$. In the same way, we can find $n-t$ elements $G_{1}, \cdots, G_{n-t}$ which form a system of parameters in $\mathfrak{N}(T)$ and which can be included in a regular system of parameters $\left\{G_{1}, \cdots, G_{n-v}\right\}$ in $\mathfrak{R}(N)$. Since $\overline{\mathfrak{N}}(M \times N)$ is a Kroneckerian product of $\overline{\mathfrak{N}}(M)$ and $\overline{\mathfrak{N}}(N)$, it follows from Lemma 4 , 33 , part I, p. 19, that we have

$$
\begin{aligned}
{[\overline{\mathfrak{N}}(M \times N)} & \left.: \Lambda\left[\left[F_{1}, \cdots, F_{m-\mu}, G_{1}, \cdots, G_{n-\nu}\right]\right]\right] \\
& =\left[\overline{\mathfrak{N}}(M): \Lambda_{M}\left[\left[F_{1}, \cdots, F_{m-\mu}\right]\right]\right] \cdot\left[\overline{\mathfrak{N}}(N): \Lambda_{N}\left[\left[G_{1}, \cdots, G_{n-\nu}\right]\right]\right]=1
\end{aligned}
$$

which proves that $F_{1}, \cdots, F_{m-\mu}, G_{1}, \cdots, G_{n-\nu}$ form a regular system of parameters in $\mathfrak{R}(M \times N)$. On the other hand, it is clear that $F_{1}, \cdots, F_{m-v}$, $G_{1}, \cdots, G_{n-t}$ form a system of parameters in $\mathfrak{R}(V \times T)$.

Let $\mathfrak{u}$ be the prime ideal which corresponds to $U$ in $K[[X]]$; let $\&$ be the prime ideal which corresponds to $S$ in $K[[Y]]$. We denote by $F_{i}^{\mathfrak{u}}$ the residue class of $F_{i}$ modulo $\mathfrak{u}$, and by $G_{j}^{8}$ the residue class of $G_{j}$ modulo 8 . It follows from Proposition 4, $\$ 3$, p. 35, that

$$
\begin{aligned}
i(M ; U \cdot V) & =e\left(\mathfrak{N}(M) / \mathfrak{u} \mathfrak{N}(M) ; F_{1}^{\mathfrak{u}}, \cdots, F_{m-v}^{\mathfrak{u}}\right) \\
& =\left[\overline{\mathfrak{N}}(M) / \mathfrak{u} \overline{\mathfrak{N}}(M): \Lambda_{M}\left[\left[F^{\mathfrak{u}}\right]\right]\right] .
\end{aligned}
$$

(28) The ring $K\left[\left[\xi_{1}, \cdots, \xi_{\mu}\right]\right]\left[\xi_{\mu+1}, \cdots, \xi_{m}\right]$ is integral over $K\left[\left[\xi_{1}, \cdots, \xi_{\mu}\right]\right]$ and is therefore complete. It is clear that $\xi_{\mu+1}, \cdots, \xi_{m}$ are not units in this ring, whence $K\left[\left[\xi_{1}, \cdots, \xi_{\mu}\right]\right]\left[\xi_{\mu+1}, \cdots, \xi_{m}\right]=K\left[\left[\xi_{1}, \cdots, \xi_{m}\right]\right]$. 
Let $\mathfrak{w}$ be the ideal generated by $\mathfrak{u}$ and $\mathfrak{s}$ in $K[[X, Y]] ; \mathfrak{w}$ is the prime ideal which corresponds to $U \times S$. We denote by $F_{i}^{\text {w }}$ and $G_{j}^{\text {w }}$ the residue classes of $F_{i}$ and $G_{j}$ modulo $\mathfrak{w}$. In the same way as above, we see that

$$
\begin{aligned}
i(N ; S \cdot T) & =\left[\overline{\mathfrak{N}}(N) / 8 \overline{\mathfrak{N}}(N): \Lambda_{N}\left[\left[G^{8}\right]\right]\right], \\
i(M \times N ;(U \times S) \cdot(V \times T)) & =\left[\overline{\mathfrak{N}}(M \times N) / \mathfrak{w} \overline{\mathfrak{N}}(M \times N): \Lambda\left[\left[F^{\mathfrak{w}}, G^{\mathfrak{w}}\right]\right]\right] .
\end{aligned}
$$

It follows from Lemma 3, $\$$, part I, p. 18, that $\overline{\mathfrak{N}}(M \times N) / \mathfrak{w} \overline{\mathfrak{N}}(M \times N)$ is a Kronecker product of $\overline{\mathfrak{N}}(M) / \mathfrak{u} \overline{\mathfrak{N}}(M)$ and $\overline{\mathfrak{N}}(N) / \mathfrak{B} \overline{\mathfrak{N}}(N)$ over $\Lambda$. The formula $i(M \times N ;(U \times S) \cdot(V \times T))=i(M ; U \cdot V) i(N ; S \cdot T)$ follows therefore immediately from Lemma 4, §3, part I, p. 19.

5. The projection formula. We consider two series of letters $\left\{X_{1}, \cdots, X_{m}\right\}$ and $\left\{Y_{1}, \cdots, Y_{n}\right\}$. Let $U$ be an algebroid variety in $E^{m+n}(X, Y)$. We denote by $0_{X}$ the origin in the space $E^{m}(X)$.

DEFINITION 1. If the intersection of $U$ and $0_{X} \times E^{n}(Y)$ has no component of dimension greater than 0 , we say that $U$ has a finite projection index with respect to $E^{m}(X)$.

This condition may also be formulated in the following way: the variety $U$ does not contain any variety $U^{\prime}$ of positive dimension such that $\operatorname{pr}_{X} U^{\prime}=0_{X}$. It follows immediately that, if $U$ has a finite projection index, then every sub-variety of $U$ has also a finite projection index.

Let $\mathfrak{u}$ be the ideal which defines $U$; then the ideal which defines $\operatorname{pr}_{x} U$ is $\mathfrak{u} \cap K[[X]]$. If we identify the residue class of an element $F(X) \in K[[X]]$ modulo $\mathfrak{u} \cap K[[X]]$ with the residue class of $F(X)$ (considered as an element of $K[[X, Y]])$ modulo $\mathfrak{u}$, we see that $\mathrm{f}\left(\operatorname{pr}_{x} U\right)$ becomes identified with a subring of $f(U)$. Let $\mathfrak{x}$ be the maximal prime ideal in $f\left(\operatorname{pr}_{x} U\right)$. The subvarieties of $U$ whose projection on $E^{m}(X)$ is $0_{X}$ correspond to the prime ideals containing $x$ in $f(U)$. The condition that $U$ should have a finite projection index is therefore equivalent with the condition that the only prime ideal containing $\mathfrak{x}$ in $\mathfrak{f}(U)$ be the maximal prime ideal of $\mathfrak{f}(U)$. If this condition is satisfied, any system of parameters in $f\left(\operatorname{pr}_{x} U\right)$ is also a system of parameters in $f(U)$. In fact, we know (Proposition 7, L.R., §III, p. 703) that we can find $u$ linear combinations $x_{1}^{*}, \cdots, x_{u}{ }^{*}$ of the functions induced by $X_{1}, \cdots, X_{m}$ on $U$ which form a system of parameters in $\mathrm{f}(U)$ (where $u=\operatorname{dim} U)$. The functions induced by $X_{1}, \cdots, X_{m}$ on $U$ are therefore integral over $K\left[\left[x_{1}^{*}, \cdots, x_{u}^{*}\right]\right]$. It follows that $f\left(\operatorname{pr}_{x} U\right)$ is integral over $K\left[\left[x_{1}^{*}, \cdots, x_{u}^{*}\right]\right]$, which proves that $\operatorname{dim}\left(\operatorname{pr}_{x} U\right)=u$; our assertion follows immediately from this formula. Furthermore, we see that $f(U)$ is integral over $f\left(\operatorname{pr}_{x} U\right)$. This justifies the following definition:

Definition 2. Assume that a variety $U$ in $E^{m+n}(X, Y)$ has a finite projection index with respect to $E^{m}(X)$. Let us identify $\mathrm{f}\left(\operatorname{pr}_{X} U\right)$ with a subring of $\mathrm{f}(U)$ in the manner which was indicated above. Then the number $\left[\mathrm{f}(U): \mathfrak{f}\left(\operatorname{pr}_{x} U\right)\right]$; 
is called the projection index of $U$; this number will be denoted by $j(U ; X)$.

THEOREM 5. Assume that a variety $U$ in $E^{m+n}(X, Y)$ has a finite projection index with respect to $E^{m}(X)$. Let $V$ be a variety in $E^{m}(X)$ and assume that $M$ is a proper component of the intersection of $\operatorname{pr}_{X} U$ and $V$. If $M_{1}, \cdots, M_{o}$ are all the distinct components of the intersection of $U$ and $V \times E^{n}(Y)$ whose projection on $E^{m}(X)$ is contained in $M$, then each $M_{i}$ is a proper component of the intersection of $U$ and $V \times E^{n}(Y)$, and we have $\operatorname{pr}_{x} M_{i}=M(1 \leqq i \leqq g)$. Furthermore, the following formula holds true:

$$
j(U ; X) \cdot i\left(M ;\left(p r_{X} U\right) \cdot V\right)=\sum_{i=1}^{g} j\left(M_{i} ; X\right) \cdot i\left(M_{i} ; U \cdot\left(V \times E^{n}(Y)\right)\right) .
$$

Let $u, v$ and $\mu$ be the respective dimensions of $U, V$ and $M$. Then $\operatorname{dim} M_{i} \geqq u+v+n-(m+n)=\mu$. Since $M_{i}$ is a subvariety of $U$, it has a finite projection index on $E^{m}(X)$, whence $\operatorname{dim} M_{i}=\operatorname{dim} \operatorname{pr}_{X} M_{i} \leqq \mu$ since $\operatorname{pr}_{X} M_{i} \subset M$. It follows that $\operatorname{dim} M_{i}=\mu$, that is, that $M_{i}$ is a proper component of the intersection of $U$ and $V \times E^{n}(Y)$, and that $\operatorname{dim} \operatorname{pr}_{X} M_{i}=\mu$, whence $\operatorname{pr}_{x} M_{i}=M$.

We construct a copy $E^{m+n}\left(X^{\prime}, Y^{\prime}\right)$ of the space $E^{m+n}(X, Y)$; then $E^{m}\left(X^{\prime}\right)$ is a copy of $E_{m}(X)$ and we denote by $V^{\prime}$ the copy of $V$ in $E^{m}\left(X^{\prime}\right)$. Let $\Delta_{X, Y}$ be the diagonal of the space $E^{m+n}(X, Y) \times E^{m+n}\left(X^{\prime}, Y^{\prime}\right)$; then

$$
i\left(M_{i} ; U \cdot\left(V \times E^{n}(Y)\right)\right)=e\left(\Re_{U \times V^{\prime} \times E^{n}\left(Y^{\prime}\right)}\left(M_{i}^{\Delta_{X, Y}}\right) ; x^{\prime}-x, y^{\prime}-y\right)
$$

where $x_{k}, x_{k}^{\prime}, y_{l}, y_{l}^{\prime}$ are the functions induced on $U \times V^{\prime} \times E^{n}\left(Y^{\prime}\right)$ by $X_{k}, X_{k}^{\prime}$, $Y_{l}, Y_{l}^{\prime}$ respectively. Let $\mathfrak{u}$ be the prime ideal in $K[[X, Y]]$ which corresponds to $U$, and let $\mathfrak{v}^{\prime}$ be the prime ideal in $K\left[\left[X^{\prime}\right]\right]$ which defines $V^{\prime}$. The variety $U \times V^{\prime} \times E^{n}\left(Y^{\prime}\right)$ is defined by the ideal generated by $\mathfrak{u}$ and $\mathfrak{v}^{\prime}$ in $K\left[\left[X, Y, X^{\prime}, Y^{\prime}\right]\right]$. This last ring is identical with $K\left[\left[X, Y, X^{\prime}, Y^{\prime}-Y\right]\right]$, and $\mathfrak{u}$ and $\mathfrak{v}^{\prime}$ are contained in $K\left[\left[X, Y, X^{\prime}\right]\right]$. It follows easily that the ideal generated by $\mathfrak{u}, \mathfrak{v}^{\prime}$ and the $n$ elements $Y_{l}^{\prime}-Y_{l}(1 \leqq l \leqq n)$ is prime. This ideal defines a certain variety $W$ contained in $U \times V^{\prime} \times E^{n}\left(Y^{\prime}\right)\left({ }^{29}\right)$. The ideal generated by the $n$ elements $y_{l}^{\prime}-y_{l}$ in the ring $\mathfrak{N}_{U \times V^{\prime} \times E^{n}\left(Y^{\prime}\right)}\left(M_{l}^{\Delta X, Y}\right)$ is therefore prime. Making use of the corollary to Theorem 5 , $\$ 5$, part I, p. 25, we obtain

$$
i\left(M_{i} ; U \cdot\left(V \times E^{n}(Y)\right)\right)=e\left(\Re_{W}\left(M_{i}^{\Delta_{X, Y}}\right) ; \xi^{\prime}-\xi\right)
$$

where $\xi_{k}$ and $\xi_{k}^{\prime}$ are the functions induced by $X_{k}$ and $X_{k}^{\prime}$ on $W$. Let also $\eta_{1}, \cdots, \eta_{n}$ be the functions induced by $Y_{1}, \cdots, Y_{n}$ on $W$. It is $\operatorname{clear}\left({ }^{30}\right)$ that $f(W)=K\left[\left[\xi, \xi^{\prime}, \eta\right]\right]$ is isomorphic with $f\left(U \times V^{\prime}\right)$ and is therefore a $M_{i}^{\Delta X Y}$.

${ }^{(29)} W$ is the intersection of $U \times V^{\prime} \times E^{n}\left(Y^{\prime}\right)$ with $E^{2 n}\left(X, X^{\prime}\right) \times \Delta^{Y}$; this variety contains

$\left.{ }^{(30}\right)$ The projection of $W$ on $E^{3 n}\left(X, Y, X^{\prime}\right)$ is $U \times V^{\prime}$ and we have $j\left(W ; X, Y, X^{\prime}\right)=1$. 
Kroneckerian product of $K[[\xi, \eta]]$ and $K\left[\left[\xi^{\prime}\right]\right]$ over $K$. The elements $\eta_{l}$ are integral over $K[[\xi]]$; it follows that $\mathfrak{f}(W)$ is finite over $\mathfrak{D}=K\left[\left[\xi, \xi^{\prime}\right]\right]$. We assert that $[\mathrm{f}(W): \mathrm{o}]=j(U ; X)$. In fact, let $\left\{\phi_{1}, \cdots, \phi_{m-u}\right\}$ be a system of parameters in $K[[\xi]]$, and therefore also in $K[[\xi, \eta]]$, and let $\left\{\psi_{1}^{\prime}, \cdots, \psi_{m-0}^{\prime}\right\}$ be a system of parameters in $K\left[\left[\xi^{\prime}\right]\right]$. It follows from Lemma $4, \S 3$, part I, p. 19 , that

$$
\begin{aligned}
{\left[\mathrm{f}(W): K\left[\left[\phi, \psi^{\prime}\right]\right]\right] } & =[K[[\xi, \eta]]: K[[\phi]]] \cdot\left[K\left[\left[\xi^{\prime}\right]\right]: K\left[\left[\psi^{\prime}\right]\right]\right], \\
{\left[K\left[\left[\xi, \xi^{\prime}\right]\right]: K\left[\left[\phi, \psi^{\prime}\right]\right]\right] } & =[K[[\xi]]: K[[\phi]]] \cdot\left[K\left[\left[\xi^{\prime}\right]\right]: K\left[\left[\psi^{\prime}\right]\right]\right]
\end{aligned}
$$

whence $[\mathrm{f}(W): \mathrm{o}]=[K[[\xi, \eta]]: K[[\phi]]] /[K[[\xi]]: K[[\phi]]]=\left[\mathrm{f}(U): \mathfrak{f}\left(\operatorname{pr}_{X} U\right)\right]$ $=j(U ; X)$.

Let $\mathfrak{m}_{i}$ be the ideal which corresponds to $M_{i}{ }^{\Delta x, Y}$ in $\mathrm{f}(W)$, and set $\mathfrak{m}=\mathfrak{m}_{i} \cap \mathfrak{o}$. The ring $\mathfrak{o}$ is isomorphic with $\mathrm{f}\left(\left(\operatorname{pr}_{X} U\right) \times V^{\prime}\right)$; in this isomorphism, $\mathfrak{m}$ is associated with the prime ideal which corresponds to the subvariety $M^{\Delta^{x}}$ of $U \times V^{\prime}$ (where $\Delta_{X}$ is the diagonal of $E^{m}(X) \times E^{m}\left(X^{\prime}\right)$ ). It follows that $\mathfrak{m}$ does not depend upon $i$. Denote by $\Im$ the subring of $\mathfrak{N}_{W}\left(M_{i}^{\Delta x, Y)}\right.$ which is generated by $\mathfrak{D}_{\mathfrak{m}}$ and $\mathfrak{f}(W)$, and set $\mathfrak{m}_{i}^{*}=\mathfrak{I m}_{i}$. Then $\Im$ is finite over $\mathfrak{o}_{\mathfrak{m}}$ and is therefore a semi-local ring (Proposition 3, L.R., §II, p. 694), and $\mathfrak{m}_{i}^{*}$ is a maximal prime ideal in $\Im$. Conversely, let $\mathrm{m}^{*}$ be any maximal prime ideal in $\Im$; then $\left.\mathfrak{m}^{*}=\mathfrak{m}^{\prime}\right\}$, where $\mathfrak{m}^{\prime}$ is a prime ideal in $\mathfrak{f}(W)$ whose intersection with $\mathfrak{v}$ is $\mathfrak{m}\left({ }^{(31}\right)$. The prime ideal $\mathfrak{m}^{\prime}$ defines a subvariety $M^{\prime}$ of $W$, that is, also of $U \times V^{\prime} \times E\left(Y^{\prime}\right)$. Since $M^{\prime} \subset W$, then $n$ functions $Y_{l}^{\prime}-Y_{l}$ vanish on $M^{\prime}$; since $m$ contains $\xi_{k}^{\prime}-\xi_{k}(1 \leqq k \leqq m)$, the functions $X_{k}^{\prime}-X_{k}(1 \leqq k \leqq m)$ vanish on $M^{\prime}$ whence $M^{\prime} \subset \Delta_{X, Y}$. It follows that $M^{\prime}=\left(M^{\prime \prime}\right)^{\Delta_{X}, Y}$ where $M^{\prime \prime}$ is a subvariety of $E^{m+n}(X, Y)$ which is contained in $U$ and in $V \times E^{n}(Y)$. Since $\mathfrak{m}^{\prime} \cap_{\mathfrak{D}}=\mathfrak{m}$, we have $\operatorname{pr}_{X} M^{\prime \prime}=M$, whence $M^{\prime \prime}=M_{i}$ for some $i$. It follows that $\mathfrak{m}_{1}^{*}, \ldots, \mathfrak{m}_{0}^{*}$ are all the maximal prime ideals of $\Im$.

The ring $\mathfrak{N}_{W}\left(M_{i}{ }^{\Delta X}, Y\right)$ is identical with the ring of quotients of $\mathfrak{m}_{i}^{*}$ with respect to $\Im$. Let $\bar{\Im}$ be a completion of $\Im$, and let $\epsilon_{i}$ be the primitive idempotent which corresponds to $m_{i}^{*} \bar{\Im}$ in $\bar{\Im}\left({ }^{32}\right)$. Then there exists an isomorphism of $\overline{\mathfrak{N}}_{W}\left(M_{i}{ }^{\Delta x, Y}\right)$ with $\bar{\Im} \epsilon_{i}$ which maps every element $\xi$ of $\Im$ upon $\xi \epsilon_{i}\left({ }^{33}\right)$. We have

$$
i\left(M_{i} ; U \cdot\left(V \times E^{n}(Y)\right)\right)=e\left(\epsilon \bar{\Im}_{i} ;\left(\xi^{\prime}-\xi\right) \epsilon_{i}\right) .
$$

Let $L$ be a basic field of $\mathfrak{D}$, that is, also of $\mathfrak{N}_{W}\left(M_{i}^{\Delta x, Y}\right)$. Then $L \epsilon_{i}$ is a basic field of $\bar{\Im}_{i}$, and we have

$$
\left[\bar{\Im}_{\epsilon_{i}}: L \epsilon_{i}\left[\left[\left(\xi^{\prime}-\xi\right) \epsilon_{i}\right]\right]\right]=e\left(\bar{\Im}_{\epsilon_{i}} ;\left(\xi^{\prime}-\xi\right) \epsilon_{i}\right) \cdot\left[\Im / \mathfrak{m}_{i}^{*}: L\right] .
$$

The field $\Im / \mathfrak{m}_{i}^{*}$ coincides with $\mathfrak{N}_{W}\left(M_{i}^{\Delta x, Y}\right) / \mathfrak{m}_{i} \mathfrak{N}_{W}\left(M_{i}{ }^{\Delta x, Y}\right)$. It is the field of quotients of $\mathrm{f}(W) / \mathfrak{m}_{i}=\mathfrak{f}\left(M_{i} \Delta_{X}, Y\right)$, which is isomorphic with $\mathfrak{f}\left(M_{i}\right)$. Similarly,

(31) $\Im$ may be regarded as a ring of quotients of $f(W)$.

(32) Cf. Proposition 2, L.R., §II, p. 693.

(33) Cf. my paper On the ring of quotients of a prime ideal, Bull. Amer. Math. Soc. vol. 50 (1944) p. 93. 
$0 / \mathfrak{m}$ is isomorphic with the field of quotients of $\mathfrak{f}(M)$. We have $\left[\Im / \mathfrak{m}_{i}^{*}: L\right]$ $=\left[\Im / \mathfrak{m}_{i}^{*}: \mathfrak{o} / \mathfrak{m}\right] \cdot[\mathrm{o} / \mathfrak{m}: L]=\left[\mathfrak{f}\left(M_{i}\right): \mathfrak{f}(M)\right] \cdot[\mathrm{o} / \mathfrak{m}: L]=j\left(M_{i} ; X\right) \cdot[\mathrm{o} / \mathfrak{m}: L]$.

It is clear that $\sum_{1}^{0}\left[\widehat{\Im}_{i}: L \epsilon_{i}\left[\left[\left(\xi^{\prime}-\xi\right) \epsilon_{i}\right]\right]\right]=\left[\widehat{\Im}: L\left[\left[\xi^{\prime}-\xi\right]\right]\right]$. Let $\left\{\alpha_{1}, \cdots, \alpha_{d}\right\}$ be a maximal system of elements of $\Im$ which are linearly independent with respect to $\mathrm{o}_{\mathfrak{m}}$; it follows easily from Proposition 7, L.R., §II, p. 699 , that $\left\{\alpha_{1}, \cdots, \alpha_{d}\right\}$ is a maximal system of elements of $\bar{\Im}$ which are linearly independent over $\overline{\mathfrak{D}}_{\mathfrak{m}}$ and that $\left[\overline{\mathfrak{F}}_{\mathrm{w}}: L\left[\left[\xi^{\prime}-\xi\right]\right]\right]=d \cdot\left[\overline{\mathrm{o}}_{\mathfrak{m}}: L\left[\left[\xi^{\prime}-\xi\right]\right]\right]$ (where $\overline{\mathfrak{D}}_{\mathfrak{m}}$ is the adherence of $\mathfrak{D}_{\mathfrak{m}}$ in $\overline{\mathfrak{\Im}}$ and is therefore a completion of $\overline{\mathfrak{D}}_{\mathfrak{m}}$ ). It follows that

$$
\left[\overline{\mathfrak{F}}: L\left[\left[\xi^{\prime}-\xi\right]\right]\right]=\left[\mathfrak{\Im}: \mathfrak{o}_{m}\right] \cdot e\left(\mathrm{o}_{m} ; \xi^{\prime}-\xi\right)[\mathrm{o} / \mathfrak{m}: L] .
$$

The number $\left[\Im: \mathbb{D}_{\mathfrak{m}}\right]$ is equal to $[\mathrm{f}(W): \mathrm{o}]$, that is, to $j(U ; X)$ as we have shown above. The number $e\left(\mathrm{o}_{\mathfrak{m}} ; \xi^{\prime}-\xi\right)$ is equal to $i\left(M ;\left(\operatorname{pr}_{X} U\right) \cdot V\right)$. It follows that $j(U ; X) i\left(M ;\left(\operatorname{pr}_{X} U\right) \cdot V\right)[\mathrm{o} / \mathfrak{m}: L]$

$$
=\sum_{i=1}^{g} i\left(M_{i} ; U \cdot\left(V \times E^{n}(Y)\right)\right) \cdot j\left(M_{i} ; X\right) \cdot[\mathrm{o} / \mathrm{m}: L] .
$$

Theorem 5 is thereby proved.

6. Associativity of intersections. Let $U, V$ and $W$ be three algebroid varieties in $E^{n}(X)$. By a component of the intersection of $U, V$ and $W$ we mean a variety $M$ which is contained in $U$, in $V$, and in $W$ but is such that no variety containing $M$ and different from $M$ itself has the same property. The components of the intersection of $U, V$, and $W$ are clearly the components of the intersections of $U$ with the components of the intersections of $V$ and $W$. Let $u, v$, and $w$ be the dimensions of $U, V$, and $W$ respectively; it follows immediately from Theorem $1, \S 2$, p. 31 , that the dimension of any component of the intersection of $U, V$, and $W$ is at least $u+v+w-2 n$. If one of these components is of dimension exactly $u+v+w-2 n$, we say that it is a proper component of the intersection of $U, V$, and $W$. Let us introduce two copies $E^{n}\left(X^{\prime}\right)$ and $E^{n}\left(X^{\prime \prime}\right)$ of the space $E^{n}(X)$; let $V^{\prime}$ be the copy of $V$ in $E^{n}\left(X^{\prime}\right)$ and let $W^{\prime \prime}$ be the copy of $W$ in $E^{n}\left(X^{\prime \prime}\right)$. The $2 n$ elements $X_{k}^{\prime}-X_{k}, X_{k}^{\prime \prime}-X_{k}(1 \leqq k \leqq n)$ of $K\left[\left[X, X^{\prime}, X^{\prime \prime}\right]\right]$ generate a prime ideal $\mathrm{b}$; let $\Delta$ be the corresponding subvariety of $E^{3 n}\left(X, X^{\prime}, X^{\prime \prime}\right)$. Assume that $M$ is a proper component of the intersection of $U, V$ and $W$, and let $\mathfrak{m}$ be the prime ideal in $K[[X]]$ which corresponds to $M$. We see easily that the ideal generated by $\mathfrak{m}$ and $\mathfrak{b}$ in $K\left[\left[X, X^{\prime}, X^{\prime \prime}\right]\right]$ is prime; it defines a variety which we may denote by $M^{\Delta}$. This variety is a subvariety of $U \times V^{\prime} \times W^{\prime \prime}$, and we see easily that it is a proper component of the intersection of $U \times V^{\prime} \times W^{\prime \prime}$ with $\Delta$ (cf. proof of Theorem 1, $\S 2$, p. 31). Let $x_{k}, x_{k}^{\prime}, x_{k}^{\prime \prime}$ be the functions induced on $U \times V^{\prime} \times W^{\prime \prime}$ by $X_{k}, X_{k}^{\prime}, X_{k}^{\prime \prime}$ respectively. The $2 n$ elements $x_{k}^{\prime}-x_{k}, x_{k}^{\prime \prime}-x_{k}$ are seen to form a system of parameters in $\mathfrak{N}_{U \times V^{\prime} \times W^{\prime \prime}}\left(M^{\Delta}\right)$ (here again, cf. proof of Theorem 1, \$2, p. 31). The multiplicity 
$i(M ; U \cdot V \cdot W)$ of $M$ in the intersection of $U, V$ and $W$ is defined to be the number

$$
i(M ; U \cdot V \cdot W)=e\left(\Re_{U \times V^{\prime} \times W^{\prime \prime}}\left(M^{\Delta}\right) ; x^{\prime}-x, x^{\prime \prime}-x\right) .
$$

The ideal generated by the elements $x_{k}^{\prime}-x_{k}, x_{k}^{\prime \prime}-x_{k}$ in the ring $\Re_{U \times V^{\prime} \times W^{\prime \prime}}\left(M^{\Delta}\right)$ coincides with the ideal generated by the elements $x_{k}^{\prime}-x_{k}, x_{k}^{\prime \prime}-x_{k}^{\prime}$ and also with the ideal generated by the elements $x_{k}^{\prime \prime}-x_{k}^{\prime}, x_{k}^{\prime \prime}-x_{k}$. It follows immediately that $i(M ; U \cdot V \cdot W)$ does not change if we permute $U, V, W$ in any way (cf. Proposition 3 , §2, part I, p. 14).

THEOREM 6. Let $U, V$ and $W$ be three algebroid varieties in $E^{n}(X)$, and assume that $M$ is a proper component of the intersection of $U, V$, and $W$. Let $P_{1}, \cdots, P_{0}$ be the components of the intersection of $U$ and $V$ which contain $M$; then each $P_{i}$ is a proper component of the intersection of $U$ and $V$, and $M$ is a proper component of the intersection of $P_{i}$ and $W$. Moreover, we have

$$
i(M ; U \cdot V \cdot W)=\sum_{i=1}^{g} i\left(P_{i} ; U \cdot V\right) i\left(M ; P_{i} \cdot W\right) .
$$

We introduce three different copies $E^{n}\left(X^{\prime}\right), E^{n}\left(X^{\prime \prime}\right)$, and $E^{n}\left(X^{\prime \prime \prime}\right)$ of the space $E^{n}(X)$. We denote by $V^{\prime}$ the copy of $V$ in $E^{n}\left(X^{\prime}\right)$ and by $W^{\prime \prime}$ the copy of $W$ in $E^{n}\left(X^{\prime \prime}\right)$. Let $R$ be the variety $U \times V^{\prime} \times W^{\prime \prime} \times E^{n}\left(X^{\prime \prime}\right)$. If $m$ is the prime ideal in $K[[X]]$ which corresponds to $M$, the ideal generated by $\mathfrak{m}$ and the $3 n$ elements $X_{k}^{\prime}-X_{k}, X_{k}^{\prime \prime}-X_{k}, X_{k}^{\prime \prime \prime}-X_{k}(1 \leqq k \leqq n)$ in $K\left[\left[X, X^{\prime}, X^{\prime \prime}, X^{\prime \prime \prime}\right]\right]$ is prime and defines a subvariety of $M^{*}$ of $R$. The projection of $M^{*}$ on $E^{3 n}\left(X, X^{\prime}, X^{\prime \prime}\right)$ is clearly the variety which was denoted above by $M^{\Delta}$; moreover, we have $j\left(M^{*} ; X, X^{\prime}, X^{\prime \prime}\right)=1$. Let $\Delta^{*}$ be the subvariety of $E^{4 n}\left(X, X^{\prime}, X^{\prime \prime}, X^{\prime \prime \prime}\right)$ which corresponds to the ideal generated by the $3 n$ elements $X_{k}^{\prime}-X_{k}, X_{k}^{\prime \prime}-X_{k}, X_{k}^{\prime \prime}-X_{k}(1 \leqq k \leqq n)$. Then the projection of $\Delta^{*}$ on $E^{3 n}\left(X, X^{\prime}, X^{\prime \prime}\right)$ is the variety which was denoted above by $\Delta$, and we have $j\left(\Delta^{*} ; X, X^{\prime}, X^{\prime \prime}\right)=1$.

By Theorem 5, $\S 5$, p. 42 , we have

$$
i\left(M^{\Delta} ;\left(U \times V^{\prime} \times W^{\prime \prime}\right) \cdot \Delta\right)=i\left(M^{*} ; R \cdot \Delta^{*}\right) .
$$

It follows immediately from Proposition $4, \S 3$, p. 35, that $i\left(M^{\Delta} ;\left(U \times V^{\prime} \times W^{\prime \prime}\right)\right.$ $. \Delta)=i(M ; U \cdot V \cdot W)$ (cf. proof of Proposition 5, §3, p. 36). Moreover, it follows from the same source that $i\left(M^{*} ; R \cdot \Delta^{*}\right)=e\left(\mathfrak{N}_{R}\left(M^{*}\right) ; x^{\prime}-x, x^{\prime \prime}-x^{\prime}\right.$, $\left.x^{\prime \prime \prime}-x^{\prime \prime}\right)$ where $x_{k}, x_{k}^{\prime}, x_{k}^{\prime \prime}, x_{k}^{\prime \prime \prime}$ are the functions induced on $R$ by $X_{k}, X_{k}^{\prime}$ $X_{k}^{\prime \prime}, X_{k}^{\prime \prime \prime}$ respectively.

Let $p_{i}$ be the dimension of $P_{i}$; then $p_{i} \geqq u+v-n$, and, since $M$ is a component of the intersection of $P_{i}$ and $W, \operatorname{dim} M \geqq p_{i}+w-n$. Since $\operatorname{dim} M$ $=u+v+w-2 n$, we conclude that $p_{i}=u+v-n$ and that $\operatorname{dim} M=p_{i}+w-n$. Therefore, $P_{i}$ is a proper component of the intersection of $U$ and $V$ and $M$ is a proper component of the intersection of $P_{i}$ and $W$. 
Denote by $\Delta_{0,1}$ the diagonal of the space $E^{n}(X) \times E^{n}\left(X^{\prime}\right)$ and by $\Delta_{2,3}$ the diagonal of the space $E^{n}\left(X^{\prime \prime}\right) \times E^{n}\left(X^{\prime \prime \prime}\right)$. Set $P_{i}^{*}=\left(P_{i}\right)^{\Delta_{0,1}} \times\left(W^{\prime \prime}\right)^{\Delta_{2,3}}$. Then $P_{i}^{*}$ is a subvariety of $R$; let $p_{i}$ be the prime ideal in $\mathrm{f}(R)$ which corresponds to $P_{i}^{*}$. We have $M^{*} \subset P_{i}^{*}$, and therefore $\mathfrak{p}_{i} \Re_{R}\left(M^{*}\right)$ is a prime ideal in $\mathfrak{N}_{R}\left(M^{*}\right)$. This prime ideal obviously contains the $2 n$ elements $x_{k}^{\prime}-x_{k}, x_{k}^{\prime \prime \prime}-x_{k}^{\prime \prime}$ $(1 \leqq k \leqq n)$. Its dimension is equal to $\operatorname{dim} P_{i}^{*}-\operatorname{dim} M^{*}=p_{i}+w-(u+v+w$ $-2 n)=n$; it follows that $\mathfrak{p}_{i} \mathfrak{N}_{R}\left(M^{*}\right)$ is a minimal prime divisor of the ideal generated by $x_{1}^{\prime}-x_{1}, \cdots, x_{n}^{\prime}-x_{n}, x_{1}^{\prime \prime \prime}-x_{1}^{\prime \prime}, \cdots, x_{n}^{\prime \prime \prime}-x_{n}^{\prime \prime}$ in $\mathfrak{N}_{R}\left(M^{*}\right)$ (cf. corollary to Theorem $2, \S 1$, part I, p. 11). Tracing our steps back, it is easy to see that conversely every minimal prime divisor of the ideal generated by $x_{1}^{\prime}-x_{1}, \cdots, x_{n}^{\prime}-x_{n}, x_{1}^{\prime \prime \prime}-x_{1}^{\prime \prime}, \cdots, x_{n}^{\prime \prime \prime}-x_{n}^{\prime \prime}$ coincides with one of the ideals $\mathfrak{p}_{i} \Re_{R}\left(M^{*}\right)$.

The ring of quotients of $\mathfrak{p}_{i} \mathfrak{N}_{R}\left(M^{*}\right)$ with respect to $\mathfrak{N}_{R}\left(M^{*}\right)$ is $\mathfrak{N}_{R}\left(P_{i}^{*}\right)$. The ring $\mathfrak{N}_{R}\left(M^{*}\right) / \mathfrak{p}_{i} \mathfrak{N}_{R}\left(M^{*}\right)$ is isomorphic with $\mathfrak{N}_{P_{i}^{*}}\left(M^{*}\right)$. Making use of Theorem $5, \S 5$, part I, p. 25, we get

$$
i(M ; U \cdot V \cdot W)=\sum_{i=1}^{o} e\left(\Re_{P_{i}} \cdot\left(M^{*}\right) ; \xi^{\prime \prime}-\xi^{\prime}\right) e\left(\mathfrak{N}_{R}\left(P_{i}^{*}\right) ; x^{\prime}-x, x^{\prime \prime \prime}-x^{\prime \prime}\right)
$$

where $\xi_{k}^{\prime}$ and $\xi_{k}^{\prime \prime}$ are the functions induced on $P_{i}^{*}$ by $X_{k}^{\prime}$ and $X_{k}^{\prime \prime}$ respectively. Making use again of Proposition 4, $\$ 3$, p. 35, we see that $e\left(\Re_{P_{i}^{*}}\left(M^{*}\right) ; \xi^{\prime \prime}-\xi^{\prime}\right)$ is equal to $i\left(M^{*} ; P_{i}^{*} \cdot\left(\Delta_{1,2} \times E^{n}(X) \times E^{n}\left(X^{\prime \prime \prime}\right)\right)\right)$, where $\Delta_{12}$ is the diagonal of the space $E^{n}\left(X^{\prime}\right) \times E^{n}\left(X^{\prime \prime}\right)$. If we project $E^{4 n}\left(X, X^{\prime}\right.$, $\left.X^{\prime \prime}, X^{\prime \prime \prime}\right)$ on $E^{2 n}\left(X^{\prime}, X^{\prime \prime}\right)$, it is clear that the only subvariety of $P_{i}^{*}$ whose projection is the origin of $E^{2 n}\left(X^{\prime}, X^{\prime \prime}\right)$ is the origin of $E^{4 n}\left(X, X^{\prime}, X^{\prime \prime}, X^{\prime \prime \prime}\right)$. It follows that $\operatorname{dim} \operatorname{pr}_{X^{\prime}, X^{\prime \prime}} P_{i}^{*}=\operatorname{dim} P_{i}^{*}$. Let $P_{i}^{\prime}$ be the copy of $P_{i}$ in $E^{n}\left(X^{\prime}\right)$; we see immediately that any element of $K\left[\left[X^{\prime}, X^{\prime \prime}\right]\right]$ which vanishes on $P_{i}^{\prime} \times W^{\prime \prime}$ also vanishes on $P_{i}^{*}$; it follows that $\operatorname{pr}_{X^{\prime}, X^{\prime \prime}} P_{i}^{*} \subset P_{i}^{\prime} \times W^{\prime \prime}$. Since $\operatorname{dim} P_{i}^{*}=\operatorname{dim} P_{i}^{\prime} \times W^{\prime \prime}$, we have $\operatorname{pr}_{X^{\prime}, X^{\prime \prime}} P_{i}^{*}=P_{i}^{\prime} \leqq W^{\prime \prime}$. It follows immediately from the fact that $X_{k}^{\prime}-X_{k}$ and $X_{k}^{\prime \prime \prime}-X_{k}^{\prime \prime}$ vanish on $P_{i}^{*}$ that $j\left(P_{i}^{*} ; X^{\prime}, X^{\prime \prime}\right)=1$. By similar arguments, we see that the projection of $M^{*}$ on $E^{2 n}\left(X^{\prime}, X^{\prime \prime}\right)$ is $\left(M^{\prime}\right)^{\Delta_{1,2}}$, where $M^{\prime}$ is the copy of $M$ in $E^{n}\left(X^{\prime}\right)$. It is clear that the only component of the intersection of $P_{i}^{*}$ and $\Delta_{1,2} \times E^{n}(X) \times E^{n}\left(X^{\prime \prime \prime}\right)$ whose projection on $E^{2 n}\left(X^{\prime}, X^{\prime \prime}\right)$ is $\left(M^{\prime}\right)^{\Delta_{1,2}}$ is $M^{*}$. Therefore, it follows from Theorem 5, §5, p. 42, that $e\left(\mathfrak{N}_{P} \cdot\left(M^{*}\right) ; \xi^{\prime \prime}-\xi^{\prime}\right)=i\left(\left(M^{\prime}\right)^{\Delta_{1,2}} ;\left(P_{i}^{\prime} \times W^{\prime \prime}\right) \cdot \Delta_{1,2}\right)$ $=i\left(M ; P_{i} \cdot W\right)$.

Making use once more of Proposition 4, $\$ 3$, p. 35, we see that $e\left(\mathfrak{R}_{R}\left(P_{i}^{*}\right) ; x^{\prime}-x, x^{\prime \prime \prime}-x^{\prime \prime}\right)=i\left(P_{i}^{*} ; R \cdot\left(\Delta_{0,1} \times \Delta_{23}\right)\right)$. The projection of $\Delta_{0,1} \times \Delta_{2,3}$ on the space $E^{3 n}\left(X, X^{\prime}, X^{\prime \prime}\right)$ is $\Delta_{0,1} \times E^{n}\left(X^{\prime \prime}\right)$; the projection of $P_{i}^{*}$ on the same space is $P_{i} \Delta_{0,1} \times W^{\prime \prime}$, and we have obviously $j\left(\Delta_{0,1} \times \Delta_{2,3} ; X, X^{\prime}, X^{\prime \prime}\right)=1$, $j\left(P_{i}^{*} ; X, X^{\prime}, X^{\prime \prime}\right)=1$. Moreover, $P_{i}^{*}$ is the only component of the intersection of $R$ and $\Delta_{0,1} \times \Delta_{2,3}$ whose projection is $P_{i}{ }^{\Delta, 1} \times W^{\prime \prime}$. It follows from Theorem 5, $\S 5$, p. 42 , that $e\left(\mathfrak{N}_{R}\left(P_{i}^{*}\right) ; x^{\prime}-x, x^{\prime \prime \prime}-x^{\prime \prime}\right)=i\left(P_{i}^{\Delta_{0,1}} \times W^{\prime \prime} ; \quad\left(U \times V^{\prime} \times W^{\prime \prime}\right)\right.$ $\cdot\left(\Delta_{0,1} \times E^{n}\left(X^{\prime \prime}\right)\right)$. Now $W^{\prime \prime}$ may be considered as a proper component of the 
intersection of $W^{\prime \prime}$ and $E^{n}\left(X^{\prime \prime}\right)$, and, as such, it has the multiplicity 1 . Therefore, it follows from Theorem $4, \S 4$, p. 39 that

$$
\begin{aligned}
i\left(P_{i}^{\Delta_{0,1}} \times W^{\prime \prime} ;\left(U \times V^{\prime} \times W^{\prime \prime}\right) \cdot\left(\Delta_{0,1} \times E^{n}\left(X^{\prime \prime}\right)\right)\right) & =i\left(P_{i} ;\left(U \times V^{\prime}\right) \cdot \Delta_{0,1}\right) \\
& =i\left(P_{i} ; U \cdot V\right) .
\end{aligned}
$$

Theorem 6 is thereby proved.

7. Algebroid hypersurfaces. Now we propose to study the algebroid varieties of dimension $n-1$ in $E^{n}(X)$.

Proposition 1. Let $K$ be a field which contains infinitely many elements, and let $X_{1}, \cdots, X_{n}$ be $n$ letters. Then the theorem of unique factorization in prime elements holds in $K\left[\left[X_{1}, \cdots, X_{n}\right]\right]\left({ }^{(34)}\right.$.

We proceed by induction on $n$. Our assertion is obvious if $n=1$. Assume that $n>1$ and that our assertion holds for $n-1$. We observe first that it follows that a ring of power series in $n-1$ letters with coefficients in $K$ is integrally closed in its field of quotients. Now, let $p$ be a prime ideal of dimension $n-1$ in $K\left[\left[X_{1}, \cdots, X_{n}\right]\right]$. Making use of Proposition 7, L.R., §III, p. 703 we see that there exist $n-1$ linear combinations $X_{1}^{*}, \cdots, X_{n-1}^{*}$ of $X_{1}, \cdots, X_{n}$ with coefficients in $K$ whose residue classes $x_{1}^{*}, \cdots, x_{n-1}^{*}$ form a system of parameters in $K[[X]] / \mathfrak{p}$. Let $X_{n}^{*}$ be a linear combination of $X_{1}, \cdots, X_{n}$ which is linearly independent of $X_{1}^{*}, \cdots, X_{n-1}^{*}$, and let $x_{n}^{*}$ be the residue class of $X^{*}$ modulo $p$. Then $x_{n}^{*}$ is integral over $K\left[\left[x_{1}^{*}, \cdots, x_{n-1}^{*}\right]\right]$. Since this last ring is integrally closed in its field of quotients, $x_{n}^{*}$ is a zero of a polynomial $F\left(x_{1}^{*}, \cdots, x_{n-1}^{*}, T\right) \in K\left[\left[x^{*}, \cdots, x_{n-1}^{*}\right]\right][T]$ whose leading coefficient is 1 and which is irreducible in $K\left(\left(x^{*}, \cdots, x_{n-1}^{*}\right)\right)[T]$. Then $F\left(X_{1}^{*}, \cdots, X_{n-1}^{*}, X_{n}^{*}\right)$ is a power series $F^{*} \in K\left[\left[X_{1}, \cdots, X_{n}\right]\right]$ and belongs to $\mathfrak{p}$. We shall prove that $F^{*}$ is a generator of $\mathfrak{p}$. Let $G$ be any element of $\mathfrak{p}$; we may represent $G$ in the form of a power series $G^{*}\left(X_{1}^{*}, \ldots, X_{n}^{*}\right)$ in $X_{1}^{*}, \cdots, X_{n}^{*}$, and we have $G^{*}\left(x_{1}^{*}, \cdots, x_{n}^{*}\right)=0$. Let $f$ be the ideal generated by $F^{*}$, and let $\bar{x}_{k}^{*}$ be the class of $X_{k}^{*}$ modulo $\mathfrak{f}(1 \leqq k \leqq n)$. Since $\mathfrak{f} \subset \mathfrak{p}$, there exists a continuous homomorphism of $K[[X]] / \mathfrak{f}$ onto $K[[X]] / \mathfrak{p}$ which maps $\bar{x}_{k}^{*}$ upon $x_{k}^{*}(1 \leqq k \leqq n)$. If $d$ is the degree of $F$ with respect to $T$, every element of $K[[X]] / /$ may be represented as a linear combination of $1, \bar{x}_{n}^{*}, \cdots, \bar{x}_{n}^{* d-1}$ with coefficients in the ring $K\left[\left[\bar{x}_{1}^{*}, \cdots, \bar{x}_{n-1}^{*}\right]\right]$ and every element of $K[[X]] / p$ may be represented uniquely in the form of a linear combination of $1, x_{n}^{*}, \cdots, x_{n}^{* d-1}$ with coefficients in $K\left[\left[x_{1}^{*}, \cdots, x_{n-1}^{*}\right]\right]$. It follows that our homomorphism is an isomorphism and that $p=K[[X]] F^{*}$.

Now, let $G$ be any irreducible element in $K[[X]]$. Then $G$ can be included in a system of parameters in $K[[X]]$ (Lemma $3, \S 1$, part I, p. 5) and it follows that any minimal prime divisor $\mathfrak{p}$ of $K[[X]] G$ is of dimension $n-1$

${ }^{(34)}$ This proposition is usually deduced from the Weierstrass preparation theorem. The result which we use here (Proposition 7, L.R., $\S I I$, p. 703) is easily seen to be a generalization of the preparation theorem. 
(cf. corollary to Theorem $2, \S 1$, part I, p. 11). We have $\mathfrak{p}=K[[X]] P$, which shows that $G$ is a multiple of $P$. Since $G$ is irreducible, we have $G=E P$, where $E$ is a unit, and $K[[X]] G=p$. It follows that if $G$ divides a product of two elements in $K[[X]]$, then it divides one of them.

Let $H$ be any element not equal to 0 in $K[[X]]$. Call the leading form of $H$ the sum of the terms of smallest degree in $H$. Then, if $H$ is decomposed into the product $H^{\prime} H^{\prime \prime}$ of two elements in $K[[X]]$, the leading form of $H$ is the product of the leading forms of $H^{\prime}$ and $H^{\prime \prime}$, and these leading forms are of degrees smaller than the leading form of $H$ unless $H^{\prime}$ or $H^{\prime \prime}$ is a unit. It follows immediately that every element can be decomposed in at least one way into a product of units or irreducible elements. This result, combined with the result which was proved above, shows that the unique factorization theorem holds in $K[[X]]$. At the same time, we have seen that every prime ideal of dimension $n-1$ in $K[[X]]$ is principal.

Returning to the case where $K$ is algebraically closed, we introduce the following definition:

DeFINITION 1. An algebroid variety of dimension $n-1$ in $E^{n}(X)$ is called an algebroid hypersurface.

It follows that the prime ideal which corresponds to an algebroid hypersurface is principal. If $F$ is a generator of this ideal, we shall say that $F=0$ is an equation of the hypersurface, or that the hypersurface is represented by the equation $\left.F=0 .{ }^{(35}\right)$.

Proposition 2. Let $S$ be a hypersurface in $E^{n}(X)$, represented by an equation $F=0$. Let $U$ be any algebroid variety in $E^{n}(X)$. If $U$ is not contained in $S$, every component of the intersection of $U$ and $S$ is proper; if $M$ is one of these components, we have $i(M ; U \cdot S)=e\left(\mathfrak{R}_{M}(U) ; F^{U}\right)$, where $F^{U}$ is the function induced by Fon $U$.

Let $u$ be the dimension of $U$; if $U$ is not contained in $S$, any component of the intersection of $S$ and $U$ is of dimension not greater than $u-1=u$. $+(n-1)-n$, and is therefore proper.

Let us introduce a new letter $Y$. The power series $Y-F(X)$ is clearly irreducible in $K[[X, Y]]$. We denote by $S^{\prime}$ the hypersurface represented by the equation $Y-F(X)=0$. We have clearly $\operatorname{pr}_{X} S^{\prime}=E^{n}(X), j\left(S^{\prime} ; X\right)=1$. The variety $U$ may be considered as the only component of the intersection of $U$ and $E^{n}(X)$ and $i\left(U ; U \cdot E^{n}(X)\right)=1$. Therefore, it follows from Theorem 5, $\$ 5$, p. 42 that the intersection of $U \times E^{1}(Y)$ and $S^{\prime}$ has only one component, which we call $U^{\prime}$, and that $i\left(U^{\prime} ;\left(U \times E^{1}(Y)\right) \cdot S^{\prime}\right)=1, \operatorname{pr}_{X} U^{\prime}=U, j\left(U^{\prime} ; X\right)=1$. Let $L$ be the hypersurface defined by the equation $Y=0$ in $E^{n+1}(X, Y)$. We set $M^{\prime}=M \times 0_{Y}$, where $0_{Y}$ is the origin in $E^{1}(Y)$. Then $M^{\prime}$ is contained in

${ }^{(35)}$ Following the terminology of A. Weil, we do not say that for instance $F^{2}=0$ is an equation of the hypersurface. 
the intersection of $U^{\prime}$ and $L$, and a comparison of dimensions shows immediately that $M^{\prime}$ is a proper component of this intersection. The intersection of $L$ and $S^{\prime}$ has only one component which is $S \times 0_{Y}$, and this component is of multiplicity 1 , as follows for instance from Proposition $6, \S 3$, p. 36. The number $i\left(M^{\prime} ;\left(S \times 0_{Y}\right) \cdot\left(U \times E^{1}(Y)\right)\right.$ is equal to $i(M ; U \cdot S)$ by Theorem 5 , $\S 5$, p. 42. Making use of Theorem $6, \S 6$, p. 45 , we obtain $i(M ; U \cdot S)$ $=i\left(M^{\prime} ; U^{\prime} \cdot L\right)$. By Proposition 4, 33 , p. 35, we have $i\left(M^{\prime} ; U^{\prime} \cdot L\right)$ $\cdot e\left(\mathfrak{N}_{U^{\prime}}\left(M^{\prime}\right) ; Y^{U^{\prime}}\right)=e\left(\mathfrak{\Re}_{U^{\prime}}\left(M^{\prime}\right) ; F^{U^{\prime}}\right)$, where $Y^{U^{\prime}}, F^{U^{\prime}}$ are the functions induced by $Y$ and $F$ on $U^{\prime}$ (we have $Y^{U^{\prime}}=F^{U^{\prime}}$ because $U^{\prime} \subset S^{\prime}$ ). Since $j\left(U^{\prime} ; X\right)=1$, $\mathrm{f}\left(U^{\prime}\right)$ is isomorphic with $\mathrm{f}(U)$ under an isomorphism which maps $F^{U^{\prime}}$ upon $F^{U}$ and the ideal which corresponds to $M^{\prime}$ upon the ideal which corresponds to $M$. It follows that $e\left(\Re_{U^{\prime}}\left(M^{\prime}\right) ; F^{U^{\prime}}\right)=e\left(\mathfrak{R}_{U}(M) ; F^{U}\right)$. Proposition 2 is proved.

If $M$ is a proper component of the intersection of a variety $U$ and a hypersurface, the ring $\mathfrak{N}_{M}(U)$ is a geometric local ring of dimension 1 . We shall now obtain some results on such rings, in connection with valuation theory.

Let first 0 be a complete local ring of dimension 1 which contains no zero divisor not equal to 0 . Let $K$ be a basic field of 0 and $u$ be a parameter in $\mathbf{0}$. There exists a valuation $v_{1}$ of $K((u))$ whose valuation ring contains $K[[u]]$; we may for instance take $v_{1}(F(u))=k$ if $F(U)$ is a power series of the form $U^{k} E(U), E(0) \neq 0$. Moreover, any other valuation $v_{1}^{\prime}$ of $K((u))$ whose valuation ring contains $K[[u]]$ is of the form $a v_{1}$ with some constant $a=v_{1}^{\prime}(u)$; this follows immediately from the fact that $v_{1}^{\prime}(E(u))=0$ if $E(0) \neq 0$. Since $K[[u]]$ is a complete valuation ring, $v_{1}$ may be extended in a unique way to a valuation $v$ of the field of quotients $Z$ of $\mathbf{o}$. It is clear that $v$ is the only valuation of $Z$ whose valuation ring contains $\mathfrak{o}$. Let $\mathfrak{v}$ be the valuation ring of $v$, and let $p$ be its valuation ideal. Then the domain of values of $v$ consists of the multiples of $1 / e$, where $e$ is the number $[Z: K((u))] /[\mathfrak{v} / \mathfrak{p}: K]$. If $\mathfrak{m}$ is the ideal of nonunits in $\mathfrak{o}$, we have $[Z: K((u))]=e(\mathfrak{0} ; u)[\mathrm{o} / \mathfrak{m}: K]$. Since the numbers $[\mathfrak{v} / \mathfrak{p}: K],[0 / \mathfrak{m}: K]$ do not depend on $u$, we see that the functions $v(x)$, $e(0 ; x)$ differ only by a constant factor for all $x \in \mathfrak{m}$. In other words, there is a valuation of $Z$ which coincides with $e(\mathfrak{b} ; x)$ on $\mathrm{m}$.

Assume now that $\mathrm{D}$ is any geometric local ring of dimension 1 which has no zero divisor not equal to 0 . Let $\overline{\mathfrak{b}}$ be a completion of $\mathfrak{b}$, and let $\mathfrak{n}_{1}, \cdots, \mathfrak{n}_{o}$ be the prime divisors of the zero ideal in $\overline{\mathfrak{D}}$. Then $\boldsymbol{D}$ is mapped isomorphically by the natural mapping of $\overline{\mathfrak{D}}$ onto $\overline{\mathrm{b}} / \mathfrak{n}_{i}$. Let $\bar{v}_{i}$ be the valuation of $\mathfrak{0} / \mathfrak{n}_{i}$ such that $\bar{v}_{i}(\bar{x})=e\left(\overline{\mathfrak{b}} / \mathfrak{n}_{i} ; \bar{x}\right)$ for every nonunit $\bar{x}$ in $\overline{\mathfrak{b}} / \mathfrak{n}_{i}$. Then there corresponds to $\bar{v}_{i}$ a valuation $v_{i}$ of $\mathfrak{D}$. Since $\mathfrak{D}$ is a geometric local ring, we have $e(\mathfrak{D} ; x)$ $=\sum_{i=1}^{o} e\left(\overline{\mathfrak{D}} / \mathfrak{n}_{i} ; \bar{x}\right)$ where $x$ is a nonunit in $\mathfrak{D}$ and $\bar{x}_{i}$ is the residue class of $x$ modulo $\mathfrak{n}_{i}$. It follows that $e(\mathrm{D} ; x)=\sum_{i=1}^{i} v_{i}(x)$. Conversely, every valuation $v$ of $\boldsymbol{D}$ is proportional to one of the valuations $v_{i}$. In fact, let $\mathfrak{b}$ be the valuation ring of $v$, and let $\mathfrak{b}$ be the completion of $\mathfrak{b}$ (considered as a valuation ring). Denote by $0^{*}$ the adherence of $\mathfrak{o}$ in $\mathfrak{b}$. It follows from Theorem 1, L.R., §II, p. 698 that $0^{*}$ is a homomorphic image of $\overline{\mathfrak{b}}$. Since $\boldsymbol{o}^{*}$ is clearly not of dimen- 
sion 0 , it is isomorphic with one of the rings $\overline{\mathfrak{b}} / \mathfrak{n}_{i}$, which proves our assertion.

8. Cycles.

DeFinition 1. By a cycle of dimension $u$ in $E^{n}(X)$ we mean a formal linear combination of a finite number of u-dimensional varieties in $E^{n}(X)$ with integral non-negative coefficients.

The cycles of a given dimension $u$ may therefore be added and multiplied by non-negative integers. A variety $U$ will be identified with the cycle $1 \cdot U$.

DEFINITION 2. We say that two varieties $U$ and $V$ in $E^{n}(X)$ have an intersection cycle if every component of their intersection is proper. This being the case, we set $U \cdot V=\sum i(M ; U \cdot V) M$, the sum being extended over all components $M$ of the intersection of $U$ and $V$. Let $\sum_{i} a_{i} U_{i}=X$ and $\sum_{j} b_{j} V_{j}=Y$ be two cycles (with $U_{i} \neq U_{i^{\prime}}$ for $i \neq i^{\prime}, V_{j} \neq V_{j^{\prime}}$ for $j \neq j^{\prime}$ ). If whenever $a_{i} b_{j} \neq 0$ the varieties $U_{i}$ and $V_{j}$ have an intersection cycle, then we say that the symbol $X \cdot Y$ is defined, and we set $X \cdot Y=\sum_{i, j} a_{i} b_{j} U_{i} \cdot V_{j}$ (the summation being extended to the combinations $(i, j)$ such that $\left.a_{i} b_{j} \neq 0\right)$.

Let us consider two local spaces $E^{n_{1}}\left(X_{1}\right)$ and $E^{n_{2}}\left(X_{2}\right)$. Let $X_{1}=\sum_{i} a_{i} U_{i, 1}$ be a cycle in $E^{n_{1}}\left(X_{1}\right)$ and let $X_{2}=\sum_{j} b_{j} U_{j, 2}$ be a cycle in $E^{n_{2}}\left(X_{2}\right)$. Then we denote by $X_{1} \times X_{2}$ the cycle $\sum_{i j} a_{i} b_{j} U_{i, 1} \times U_{j, 2}$ in $E^{n_{1}}\left(X_{1}\right) \times E^{n_{2}}\left(X_{2}\right)$.

Theorem $4 \mathrm{a}$. Let $X_{1}$ and $Y_{1}$ be cycles in $E^{n_{1}}\left(X_{1}\right)$ and let $X_{2}$ and $Y_{2}$ be cycles in $E^{n_{2}}\left(X_{2}\right)$. If the symbols $X_{1} \cdot Y_{1}$ and $X_{2} \cdot Y_{2}$ are defined, then $\left(X_{1} \times X_{2}\right)$ $\cdot\left(Y_{1} \times Y_{2}\right)$ is defined and is equal to $\left(X_{1} \cdot Y_{1}\right) \times\left(X_{2} \cdot Y_{2}\right)$.

This follows immediately from Theorem $4, \S 4$, p. 39 .

Remark. It is easy to see that if $\left(X_{1} \times X_{2}\right) \cdot\left(Y_{1} \times Y_{2}\right)$ is defined, then $X_{1} \cdot Y_{1}$ and $X_{2} \cdot Y_{2}$ are defined except perhaps if one of the cycles $X_{1}, X_{2}, Y_{1}, Y_{2}$ is the zero cycle.

Let now $U$ be a cycle in $E^{m}(X) \times E^{n}(Y)$, and set $U=\sum_{i} a_{i} U_{i}$. If, whenever $a_{i} \neq 0, U_{i}$ has a finite projection index on $E^{m}(X)$, we say that the symbol al.pr. $X$ is defined and equal to $\sum_{i} a_{i} j\left(U_{i} ; X\right) \operatorname{pr}_{X} U_{i}$ (the summation being extended over the indices $i$ for which $\left.a_{i} \neq 0\right)$.

THeOREM 5a. Let $U$ be a cycle in $E^{m}(X) \times E^{n}(Y)$ and let $V$ be a cycle in $E^{m}(X)$. If either one of the symbols (al.pr.x $\left.U\right) \cdot V$ and al.pr.x $\left(U \cdot\left(V \times E^{n}(Y)\right)\right.$ ) is defined, the other is also defined and both represent the same cycle.

It follows immediately from Theorem $5, \S 5$, p. 42 , that if the first one of our symbols is defined, the second one is also defined and that the symbols are equal in this case. Assume now that the second symbol is defined. Assume that a variety $U_{i}$ occurs with a coefficient not equal to 0 in $U$. If the projection on $E^{m}(X)$ of a subvariety of $U_{i}$ is the origin, this subvariety is necessarily contained in any variety of the form $V_{j} \times E(Y)$. It follows that, if $V \neq 0$ (which we may assume, since otherwise the first symbol is trivially 
defined), the symbol al.pr.x $U$ is defined. Let $U_{i}$ be a variety which occurs with a coefficient not equal to 0 in $U$ and let $V_{j}$ be a variety which occurs with a coefficient not equal to 0 in $V$; let $u$ and $v$ be the dimensions of $U$ and $V$. If $\operatorname{pr}_{X} U_{i}$ and $V_{j}$ had a variety of dimension greater than $u+v-m$ in common, this variety would be the projection of a subvariety of $U_{i}$ of dimension greater than $u+v-m$ which would also be contained in $V \times E^{n}(Y)$, which is impossible $\left({ }^{36}\right)$. It follows that the first symbol is defined.

TheOREM 6a. Let $U, V$ and $W$ be three cycles not equal to 0 in $E^{n}(X)$. If either one of the symbols $(U \cdot V) \cdot W$ or $U \cdot(V \cdot W)$ is defined, the other is also defined and both symbols represent the same cycle.

Let $u, v$, and $w$ be the respective dimensions of $U, V$, and $W$. Let $M$ be a component of the intersection of $U_{i}, V_{j}$, and $W_{k}$, where $U_{i}, V_{j}$, and $W_{k}$ are varieties which occur with coefficients not equal to 0 in $U, V$, and $W$ respectively. Then $M$ is contained in some component $P$ of the intersection of $U_{i}$ and $V_{j} ;$ if $(U \cdot V) \cdot W$ is defined, we have $\operatorname{dim} P=u+v-n, \operatorname{dim} M=(\operatorname{dim} P)$ $+w-n=u+v+w-2 n$. We see in this way that if either one of the symbols in question is defined, every component of the intersection of $U, V$, and $W$ is proper and Theorem $6 a$ then follows easily from Theorem $6, \S 6, \mathrm{p} .45$.

To every irreducible power series $F$ in $K[[X]]$, we assign the cycle $(F)=1 \cdot S$, where $S$ is the algebroid hypersurface represented by the equation $F=0$. If $(F)$ is any power series not equal to 0 , we set $(F)=\left(F_{1}\right)+\cdots+\left(F_{h}\right)$ if $F=E F_{1} \cdots F_{h}$ is a decomposition of $F$ into the product of a unit $E$ and of irreducible factors $F_{1}, \cdots, F_{h}((F)=0$ if $F$ is a unit).

If $F_{1}, \cdots, F_{g}$ are power series not equal to 0 , and if the intersection of the cycles $\left(F_{1}\right), \cdots,\left(F_{g}\right)$ is defined, we set

$$
\left(F_{1}, \cdots, F_{g}\right)=\left(F_{1}\right) \cdots\left(F_{\vartheta}\right) .
$$

If this cycle exists, it is of dimension $n-g$.

THEOREM 7. Let $U$ be a variety, and let $F_{1}, \cdots, F_{g}$ be $g$ power series. Assume that the cycle $U \cdot\left(F_{1}, \cdots, F_{g}\right)$ is defined. If $M$ is a variety which occurs with a coefficient not equal to 0 in $U \cdot\left(F_{1}, \cdots, F_{0}\right)$, then the functions $F_{1}^{U}, \cdots, F_{0}^{U}$ induced by $F_{1}, \cdots, F_{o}$ on $U$ form a system of parameters in $\mathfrak{N}_{U}(M)$ and the coefficient of $M$ in $U \cdot\left(F_{1}, \cdots, F_{g}\right)$ is $e\left(\Re_{U}(M) ; F_{1}^{U}, \cdots, F_{g}^{U}\right)$.

We proceed by induction on $g$. Assume first that $g=1$. If $F_{1}$ is irreducible, our assertion follows immediately from Proposition $2, \S 7$, p. 48 . If not, we observe that $e\left(\mathfrak{N}_{U}(M) ; F^{U}\right)=\sum_{i} v_{i}\left(F^{U}\right)$, where $v_{1}, \cdots, v_{h}$ are a certain number of valuations in $\mathfrak{N}_{U}(M)$ (cf. end of $\$ 7$, p. 49). It follows that $e\left(\Re_{U}(M) ;(F G)^{U}\right)=e\left(\mathfrak{N}_{U}(M) ; F^{U}\right) e\left(\mathfrak{\Re}_{U}(M) ; G^{U}\right)$ (where $F$ and $G$ are two non-

(36) We have already seen that $U_{1}$ has a finite projection index on $E^{m}(X)$. It follows that every subariety of $U_{1}$ has the same dimension as its projection and that every subvariety of $\operatorname{pr}_{X} U_{1}$ is the projection of some subvariety of $U_{1}$. 
units in $K[[X]]$, none of which vanishes on $U$ ). It follows immediately from this that Theorem 1 holds for $g=1$.

Assume now that Theorem 1 holds for systems of $g-1$ power series. Making use of Theorem $6 \mathrm{a}$, we have $U \cdot\left(F_{1}, \cdots, F_{g}\right)=\left(U \cdot\left(F_{1}, \cdots, F_{g-1}\right)\right) \cdot\left(F_{g}\right)$. Let $M_{1}, \cdots, M_{h}$ be the varieties containing $M$, contained in $U$, and on which $F_{1}, \cdots, F_{g-1}$ vanish. Then, by our induction assumption, the coefficient of $M$ in $U \cdot\left(F_{1}, \cdots, F_{0}\right)$ is equal to $\sum_{i=1}^{h} e\left(\mathfrak{N}_{U}\left(M_{i}\right) ; F_{1}^{U}, \cdots, F_{0-1}^{U}\right) \cdot e\left(\mathfrak{N}_{M_{i}}(M) ; F_{0}^{M_{i}}\right)$, where $F_{0}^{M_{i}}$ is the function induced on $M_{i}$ by $F_{g}$. Let $\mathfrak{m}_{i}$ be the prime ideal which corresponds to $M_{i}$ in $\mathfrak{f}(U)$; then the ideals $\mathfrak{m}_{i} \Re_{U}(M)$ are exactly all the minimal prime divisors of the ideal generated by $F_{1}^{U}, \ldots, F_{g-1}^{U}$ in $\mathfrak{N}_{U}(M)$, and $\mathfrak{R}_{U}\left(M_{i}\right)$ is the ring of quotients of $\mathfrak{m}_{i} \mathfrak{N}_{U}(M)$ with respect to $\mathfrak{R}_{U}(M)$. The ring $\mathfrak{N}_{M_{i}}(M)$ may be identified with $\mathfrak{N}_{U}(M) / \mathfrak{m}_{i} \mathfrak{N}_{U}(M)$. The ring $\mathfrak{N}_{U}(M)$ is of dimension $u-(u+n-g-n)=g$; it is clear that the only prime ideal in this ring which contains $F_{1}^{U}, \ldots, F_{0}^{U}$ is the maximal prime ideal. Therefore $F_{1}^{U}, \ldots, F_{0}^{U}$ form a system of parameters. Theorem 7 then follows immediately from Theorem 5, §5, part I, p. 25 .

9. Relative intersection multiplicities. Throughout this section, $\Omega$ will denote an algebroid variety in $E^{n}(X)$ on which we shall assume that the origin 0 is a simple point. We shall denote by $\omega$ the dimension of $\Omega$.

The ring $f(\Omega)$ is identical with $\Re_{\Omega}(0)$. Since it is a regular local ring, we conclude that $f(\Omega)$ is a ring of power series; more precisely, if $\left\{x_{1}^{*}, \cdots, x_{\omega}^{*}\right\}$ is any regular system of parameters in $f(\Omega)$, we have $f(\Omega)=K\left[\left[x^{*}\right]\right]$.

Let us introduce $\omega$ new letters $X_{1}^{*}, \cdots, X_{\omega}^{*}$. Then to every regular system of parameters $\left\{x_{1}^{*}, \cdots, x_{\omega}^{*}\right\}$ in $f(\Omega)$ there corresponds an isomorphism $J_{x^{*}}$ of $\mathrm{f}(\Omega)$ with $K\left[\left[X^{*}\right]\right]$ which maps $x_{i}^{*}$ upon $X_{i}^{*}(1 \leqq i \leqq \omega)$. This isomorphism defines a one-to-one correspondence $U \rightleftarrows J_{x^{*}}(U)$ between the subvarieties $U$ of $\Omega$ and the varieties in $E^{\omega}\left(X^{*}\right)$. We shall make use of this correspondence to study the subvarieties of $\Omega$.

If we replace $\left\{x_{1}^{*}, \cdots, x_{\omega}^{*}\right\}$ by another regular system of parameters $\left\{x_{1}^{* *}, \cdots, x_{\omega}^{* *}\right\}$, the isomorphism $J_{x^{*}}$ is replaced by another isomorphism $J_{x^{*},}$, which may be written in the form $A \circ J_{x^{*}}$, where $A$ is an automorphism of $K\left[X^{*}\right]$. The automorphism $A$ establishes a permutation $U^{*} \rightleftarrows A\left(U^{*}\right)$ of the varieties in $E^{n}\left(X^{*}\right)$ among themselves. If $U$ is any subvariety of $\Omega$, we have $J_{x^{*}}(U)=A\left(J_{x^{*}}(U)\right)$. It follows that the properties of varieties $U^{*}$ in $E^{n}\left(X^{*}\right)$ which are invariant by any automorphism $A$ will give rise to properties of the subvarieties $U$ of $\Omega$.

LEMMA 1. Let $A$ be an automorphism of the ring $K\left[\left[X_{1}, \cdots, X_{n}\right]\right]$ and let $U \rightleftarrows A(U)$ be the corresponding permutation of the varieties in $E^{n}(X)$ among themselves. Then $A(U)$ has the same dimension of $U$. If $M$ is a component of the intersection of $U$ and another variety $V$, then $A(M)$ is a component of the intersection of $A(U)$ and $A(V)$. If $M$ is proper, so is $A(M)$. If this is the case, then we have $i(M ; U \cdot V)=i(A(M) ; A(U) \cdot A(V))$. 
The first three assertions are obvious. In order to prove the fourth one, let us construct a copy $E^{n}\left(X^{\prime}\right)$ of the space $E^{n}(X)$. To the automorphism $A$ of $K[[X]]$ there corresponds an automorphism $A^{\prime}$ of $K\left[\left[X^{\prime}\right]\right]$, and there exists an automorphism $A^{*}$ of $K\left[\left[X, X^{\prime}\right]\right]$ which coincides with $A$ on $K[[X]]$ and with $A^{\prime}$ on $K\left[\left[X^{\prime}\right]\right]$. Let $V^{\prime}$ be the copy of $V$ in $E^{n}\left(X^{\prime}\right)$; then the copy of $A(V)$ is $A^{\prime}\left(V^{\prime}\right)$. We have $A^{*}\left(U \times V^{\prime}\right)=A(U) \times A^{\prime}\left(V^{\prime}\right)$. We shall see that $A^{*}\left(M^{\Delta}\right)=(A(M))^{\Delta}$. Set $F_{i}(X)=A\left(X_{i}\right)(1 \leqq i \leqq n)$, whence $F_{i}\left(X^{\prime}\right)=A^{\prime}\left(X_{i}^{\prime}\right)$. Since $A$ is an automorphism, $X_{1}, \cdots, X_{n}$ belong to $K\left[\left[F_{1}, \cdots, F_{n}\right]\right]$, from which it follows that the functional determinant of $F_{1}, \cdots, F_{n}$ is a unit in $K[[X]]$. If $X_{i}=\phi_{i}\left(F_{1}, \cdots, F_{n}\right)(1 \leqq i \leqq n)$ is the expression of $X_{i}$ as a power series in $F_{1}, \cdots, F_{n}$, we have $X_{i}^{\prime}=\phi_{i}\left(F_{1}\left(X^{\prime}\right), \cdots, F_{n}\left(X^{\prime}\right)\right)$ from which it follows immediately that the ideal generated in $K\left[\left[X, X^{\prime}\right]\right]$ by the $n$ elements $F_{i}\left(X^{\prime}\right)-F_{i}(X)(1 \leqq i \leqq n)$ coincides with the prime ideal which corresponds to $\Delta$; it follows that $A^{*}(\Delta)=\Delta$, whence $A^{*}\left(M^{\Delta}\right) \subset \Delta$. On the other hand, it is clear that $\operatorname{pr}_{X} A^{*}\left(M^{\Delta}\right)=A\left(\operatorname{pr}_{X} M^{\Delta}\right)=A(M)$, whence $A^{*}\left(M^{\Delta}\right)=(A(M))^{\Delta}$. Let $x_{i}$ and $x_{i}^{\prime}$ be the functions induced by $X_{i}$ and $X_{i}^{\prime}$ respectively on $U \times V^{\prime}$; in virtue of what we have proved, $A^{*}$ defines an isomorphism (also denoted by $\left.A^{*}\right)$ of $\mathfrak{N}_{U \times V^{\prime}}\left(M^{\Delta}\right)$ with $\mathfrak{N}_{A(U) \times A(V)}\left((A(M))^{\Delta}\right)$, and $A^{*}\left(x_{i}\right), A^{*}\left(x_{i}^{\prime}\right)$ are the functions induced by $F_{i}(X), F_{i}\left(X^{\prime}\right)$.respectively on $A(U), A(V)^{\prime}$. We have

$$
i(M ; U \cdot V)=e\left(\Re_{A(U) \times} \times_{A(V)}\left(\left(A\left(M^{\Delta}\right)\right) ; A^{*}\left(x^{\prime}\right)-A^{*}(x)\right) .\right.
$$

On the other hand, we have also seen that the ideal generated by the $n$ elements $A^{*}\left(x_{i}^{\prime}\right)-A^{*}\left(x_{i}\right)$ is the same as the ideal generated by the functions induced on $A(U) \times A(V)^{\prime}$ by the elements $X_{i}^{\prime}-X_{i}(1 \leqq i \leqq n)$. Taking Proposition 3, $\$ 2$, part I, p. 14 into account, we see that $i(M ; U \cdot V)$ $=i(A(M) ; A(U) \cdot A(V))$.

THEOREM 1a. Let $U$ and $V$ be two subvarieties of $\Omega$, of respective dimensions $u$ and $v$. Then every component of the intersection of $U$ and $V$ is of dimension at least $u+v-\omega$.

This follows immediately from Theorem $1, \S 2$, p. 31 , and from Lemma 1 .

Definition 1. Let $U$ and $V$ be two subvarieties of $\Omega$, of respective dimensions $u$ and $v$. A component $M$ of the intersection of $U$ and $V$ is said to be proper with respect to $\Omega$ if it is of dimension $u+v-\omega$. This being the case, let $J_{x^{*}}$ be an isomorphism off $f(\Omega)$ with $K\left[\left[X_{1}^{*}, \cdots, X_{\omega}^{*}\right]\right]$; then the number $i\left(J_{x^{*}}(M) ; J_{x^{*}}(U) \cdot J_{x^{*}}(V)\right)$ is called the relative multiplicity of $\dot{M}$ in the intersection of $U$ and $V$ with respect to $\Omega$. This number is denoted by $i_{\Omega}(M ; U \cdot V)$.

This definition is justified by Lemma 1 , which shows that the value of $i\left(J_{x^{*}}(M) ; J_{x^{*}}(U) \cdot J_{x^{*}}(V)\right)$ does not depend upon the choice of $J_{x^{*}}$.

Lemma 2. Let $F_{1}, \cdots, F_{n-u}$ be $n-u$ power series in $K[[X]]$ which vanish at the origin and whose Jacobian matrix is of rank $n-u$ at the origin. Then the cycle $\left(F_{1}, \cdots, F_{n-u}\right)$ is defined and represents a variety $U$ on which the origin is 
simple. If $X_{i_{1}}, \cdots, X_{i_{u}}$ are $u$ of the letters $X$ such that the functional determinant of $F_{1}, \cdots, F_{n-u}$ with respect to the letters $X$ which do not occur among $X_{i_{1}}, \cdots, X_{i_{u}}$ does not vanish at the origin, then the functions induced on $U$ by $X_{i_{1}}, \cdots, X_{i_{u}}$ form a regular system of parameters in $\mathrm{f}(U)$. The prime ideal which corresponds to $U$ is the ideal generated by $F_{1}, \cdots, F_{n-u}$. Conversely, any variety $U$ on which the origin is simple may be represented in the form $\left(F_{1}, \cdots, F_{n-u}\right)$, where $F_{1}, \cdots, F_{n-u}$ are power series whose Jacobian matrix is of rank $n-u$ at the origin.

It is clear that the functional determinant of $F_{1}, \cdots, F_{n-u}, X_{i_{1}}, \cdots, X_{i_{u}}$ with respect to $X_{1}, \cdots, X_{n}$ does not vanish at the origin. Therefore $F_{1}, \cdots, F_{n-u}, X_{i_{1}}, \cdots, X_{i_{u}}$ form a regular system of parameters in $K[[X]]$ (cf. Proposition 2, $\$ 3$, p. 34), whence $K[[X]]=K\left[\left[F_{1}, \cdots, F_{n-u}\right.\right.$, $\left.\left.X_{i_{1}}, \cdots, X_{i_{u}}\right]\right]$. The first part of Lemma 2 follows immediately from this formula. Let now $U$ be a variety of dimension $u$ on which the origin is simple. Then there exists a regular system of parameters $F_{1}, \cdots, F_{n}$ in $K[[X]]$ such that $F_{1}, \cdots, F_{n-u}$ vanish on $U$ (Proposition $3, \S 3$, p. 35). The functional determinant of $F_{1}, \cdots, F_{n}$ with respect to $X_{1}, \cdots, X_{n}$ does not vanish at the origin; it follows that the Jacobian matrix of $F_{1}, \cdots, F_{n-u}$ is of rank $n-u$ at the origin. Making use of the first part of the lemma, we see that $U^{\prime}=\left(F_{1}, \cdots, F_{n-u}\right)$ is a variety of dimension $u$. Since $U \subset U^{\prime}$, we have $U=U^{\prime}$ and Lemma 2 is proved.

We shall now present an equivalent definition of the relative intersection multiplicities.

THEOREM 8. Let $U$ be any subvariety of $\Omega$. Then there exists a variety $U_{1}$ in $E^{n}(X)$ such that $U_{1} \cdot \Omega=U$. Let $V$ be another subvariety of $\Omega$, and assume that $M$ is a proper component of the intersection of $U$ and $V$ with respect to $\Omega$. Then, if $U_{1}$ is any variety with the property indicated above, $M$ is a proper component of the intersection of $U_{1}$ and $V$ and $i_{\Omega}(M ; U \cdot V)=i\left(M ; U_{1} \cdot V\right)$.

We know by Lemma 2 that we can find $\omega$ indices $i_{1}, \cdots, i_{\omega}\left(1 \leqq i_{\lambda} \leqq n\right.$, $1 \leqq \lambda \leqq \omega)$ such that the functions $x_{1}^{*}, \cdots, x_{\omega}{ }^{*}$ induced by $X_{i_{1}}, \cdots, X_{i_{\omega}}$ on $\Omega$ form a regular system of parameters in $f(\Omega)$. We can find $n-\omega$ power series $F_{1}, \cdots, F_{n-\omega}$ in $\omega$ arguments such that $Z_{h}=X_{j_{h}}-F_{h}\left(X_{i_{1}}, \cdots, X_{i_{\omega}}\right)$ vanishes on $\Omega(1 \leqq h \leqq n-\omega) ;\left(X_{j_{1}}, \cdots, X_{j_{n-\omega}}\right.$ are the letters which do not occur among $\left.X_{i_{1}}, \cdots, X_{i_{\omega}}\right)$. We have $K[[X]]=K\left[\left[X_{i_{1}}, \cdots, X_{i_{\omega}}, Z_{1}, \cdots, Z_{n-\omega}\right]\right]$. There corresponds to the elements $x_{\lambda}{ }^{*}(1 \leqq \lambda \leqq \omega)$ an isomorphism $J_{x^{*}}$ of $\mathfrak{f}(\Omega)$ with $E^{\omega}\left(X^{*}\right)$. Introduce $n-\omega$ new letters $Z_{h}^{*}(1 \leqq h \leqq n-\omega)$, and define $\widetilde{J}$ to be the isomorphism of $K[[X]]$ with $K\left[\left[X^{*}, Z^{*}\right]\right]$ which maps $X_{i \lambda}$ upon $X_{\lambda}^{*}(1 \leqq \lambda \leqq \omega)$ and $Z_{h}$ upon $Z_{h}^{*}(1 \leqq h \leqq n-\omega)$. Then $\tilde{J}$ establishes a one-toone correspondence between the varieties in $E^{n}(X)$ and the varieties in $E^{n}\left(X^{*}, Z^{*}\right)$. Let $0_{z^{*}}$ be the origin of $E^{n-\omega}\left(Z^{*}\right)$. Then, clearly, $\tilde{J}(U)=J_{x^{*}}(U) \times 0_{Z^{*}}$ for any subvariety $U$ of $\Omega$. Let $U_{1}$ be the variety in $E^{n}(X)$ which is defined by 
the condition that $f\left(U_{1}\right)=J_{x^{*}}(U) \times E^{n-\omega}\left(Z^{*}\right)$. Then $U$ is obviously the only component of the intersection of $U_{1}$ and $\Omega$ and $i\left(U ; U_{1} \cdot \Omega\right)=1$ by Theorem 4, $\S 4$, p. 39.

Let $V_{1}$ be the variety such that $\tilde{J}\left(V_{1}\right)=J_{x^{*}}(V) \times E^{n-\omega}\left(Z^{*}\right)$ and let $U_{1}^{\prime}$ be any variety such that $U=U_{1}^{\prime} \cdot \Omega$. If $u$ and $v$ are the dimensions of $U$ and $V$ respectively, $U_{1}$ is of dimension $n+u-\omega$ and $V_{1}$ is of dimension $n+v-\omega$. Since $M$ is of dimension $u+v-\omega$, it is a proper component of the intersection of $U_{1}^{\prime}, V_{1}$ and $\Omega$. It follows from Theorem $6, \S 6$, p. 45 that $i\left(M ; U_{1}^{\prime} \cdot V\right)=i\left(M ; U \cdot V_{1}\right)$. By Theorem $4, \S 4$, p. 39, we see that $i\left(M ; U \cdot V_{1}\right)$ $=i\left(J_{x^{*}}(M) ; J_{x^{*}}(U) \cdot J_{x^{*}}(V)\right)=i_{\Omega}(M ; U \cdot V)$. Theorem 8 is thereby proved.

We shall now proceed to generalize for relative intersections the essential properties of ordinary intersections.

It would be easy to give a criterion of relative multiplicity 1 which is a straightforward generalization of Theorem $2, \S 3$, p. 37. Instead, we shall give here a theorem of a slightly different type, which will be useful in the intersection theory of algebraic varieties.

THEOREM 9. Let $U$ and $V$ be two subvarieties of $\Omega$, of respective dimensions $u$ and $v$. Then the following two assertions are equivalent: (1) there exist $n+\omega-u-v$ power series of which each vanishes either on $U$ or on $V$ and whose Jacobian matrix is of rank $n+\omega-u-v$ at the origin; (2) the intersection of $U$ and $V$ contains only one component $M$; this component is proper with respect to $\Omega$ and $i_{\Omega}(M ; U \cdot V)=1$; the origin is simple on $M$. Furthermore, if (1) and (2) hold true, then the origin is simple on both $U$ and $V$.

Assume first that (1) holds. Let $H_{k}(1 \leqq k \leqq n+\omega-u-v)$ be power series which have the described properties. By Lemma 2 above, the cycle $\left(H_{1}, \cdots, H_{n+\omega-u-v}\right)$ is defined and is a variety of $M$ of dimension $u+v-\omega$ on which the origin is simple. If $M_{0}$ is any component of the intersection of $U$ and $V$, then clearly $M_{0} \subset M$. Since $\operatorname{dim} M_{0} \geqq u+v-\omega$ by Theorem $1 \mathrm{a}$, we have $M=M_{0}$. Let $\mathfrak{u}$ and $\mathfrak{b}$ be the prime ideals which correspond to $U$ and $V$; the prime ideal $\mathfrak{m}$ which corresponds to $M$ is the ideal generated by $H_{1}, \cdots, H_{n+\omega-u-v}$ and is therefore contained in the ideal generated by $\mathfrak{u}$ and $\mathfrak{v}$. It follows immediately that $m$ coincides with the ideal generated by $\mathfrak{u}$ and $\mathfrak{b}$. Let $\mathfrak{q}$ be the prime ideal which corresponds to $\Omega$. Then $\mathfrak{m} / \mathfrak{q}$ is the ideal generated by $\mathfrak{u} / \mathfrak{q}$ and $\mathfrak{b} / \mathfrak{q}$. Making use of Proposition $6, \S 3$, p. 36 we see that $i\left(J_{x^{*}}(M) ; J_{x^{*}}(U) \cdot J_{x^{*}}(V)\right)=1$, whence $i_{\Omega}(M ; U \cdot V)=1$.

Assume now that (2) holds. Since the origin is simple on $M$, we can find $\mu$ letters $X$, say $X_{m_{1}}, \cdots, X_{m_{\mu}}$, such that the functions induced by $X_{m_{1}}, \cdots, X_{m_{\mu}}$ on $M$ form a regular system of parameters in $f(M)$ ( $\mu$ is the dimension of $M$, that is, $\mu=u+v-\omega)$. Then it is clear that $M \cdot\left(X_{m_{1}}, \cdots, X_{m_{\mu}}\right)=0$, where 0 stands for the origin. Let $U_{1}$ be a variety such that $U=U_{1} \cdot \Omega$. Then $M=U_{1} \cdot V$, whence, by Theorem 6a, $\S 8$, p. 51, $0=U_{1} \cdot V \cdot\left(X_{m_{1}}, \cdots, X_{m_{\mu}}\right)$. It follows in particular that $V \cdot\left(X_{m_{1}}, \cdots, X_{m_{\mu}}\right)$ 
is a variety on which 0 is simple (by the corollary to Theorem $2, \S 3, \mathrm{p}$. 37). It follows that we can find indices $m_{\mu+1}, \cdots, m_{\mu+\mu^{\prime}}$, such that $0=\left(V \cdot\left(X_{m_{1}}, \cdots, X_{m_{\mu}}\right)\right) \cdot\left(X_{m_{\mu+1}}, \cdots, X_{m_{\mu+\mu^{\prime}}}\right)=V \cdot\left(X_{m_{1}}, \cdots, X_{m_{\mu+\mu^{\prime}}}\right)$. This proves in particular that 0 is simple on $V$. Moreover, the functions induced by $X_{1}, \cdots, X_{m_{\mu+\mu^{\prime}}}$, on $V$ form a regular system of parameters in $\mathrm{f}(V)$. By Proposition 9, L.R., §III, p. 703, the functions induced on $V$ by $X_{m_{1}}, \cdots, X_{m_{\mu}}$ generate a prime ideal in $f(V)$. This means that the ideal generated by $\mathfrak{v}$ and $X_{m_{1}}, \cdots, X_{m_{\mu}}$ is the prime ideal $\mathfrak{b}^{\prime}$ which corresponds to the variety $V \cdot\left(X_{m_{1}}, \cdots, X_{m_{\mu}}\right)$. Let $\mathfrak{u}_{1}$ be the prime ideal which corresponds to $U_{1}$; then (Proposition 6, §3, p. 36) the ideal generated by $\mathfrak{u}_{1}$ and $\mathfrak{v}^{\prime}$ is the ideal of nonunits in $K[[X]]$. It follows that we can find $n$ elements $H_{1}^{\prime}, \cdots, H_{n}^{\prime}$ of which each belongs either to $\mathfrak{u}_{1}$ or to $\mathfrak{v}^{\prime}$ such that their functional determinant does not vanish at the origin. Arranging the power series $H_{1}^{\prime}, \cdots, H_{n}^{\prime}$ in a suitable order, we may assume without loss of generality that the functional determinant of $H_{1}^{\prime}, \cdots, H_{n-\mu}^{\prime}$ with respect to the letters $X$ other than $X_{m_{1}}, \cdots, X_{m_{\mu}}$ does not vanish at the origin. This property will be preserved if we modify $H_{1}^{\prime}, \cdots, H_{n-\mu}^{\prime}$ by adding elements of the ideal generated by $X_{m_{1}}, \cdots, X_{m_{\mu}}$. By such modifications we may bring those power series $H_{k}^{\prime}$ which are not in $\mathfrak{u}_{1}$ in the ideal $\mathfrak{b}$. We see that (1) holds. Furthermore, we have seen in the course of the proof that 0 is simple on $V$. A similar argument shows that 0 is also simple on $U$.

THeOREM $4 \mathrm{~b}$. Let us consider two series of letters $\left\{X_{1}, \cdots, X_{m}\right\}$ and $\left\{Y_{1}, \cdots, Y_{n}\right\}$. Let $\Omega$ be a variety in $E^{m}(X)$ on which the origin is simple, and let $U$ and $V$ be two subvarieties of $\Omega$. Let $\Omega_{1}$ be a variety in $E^{n}(Y)$ on which the origin is simple, and let $R$ and $S$ be two subvarieties of $\Omega_{1}$. Assume that $M$ is a proper component of the intersection of $U$ and $V$ with respect to $\Omega$ and that $N$ is a proper component of the intersection of $R$ and $S$ with respect to $\Omega_{1}$. Then $M \times N$ is a proper component of the intersection of $U \times R$ and $V \times S$ with respect to $\Omega \times \Omega_{1}$ and we have $i_{\Omega \times \Omega_{1}}(M \times N ;(U \times R) \cdot(V \times S))=i_{\Omega}(M ; U \cdot V) i_{\Omega_{1}}(N ; R \cdot S)$.

We may consider $\mathfrak{f}\left(\Omega \times \Omega_{1}\right)$ as a Kroneckerian product of $\mathfrak{f}(\Omega)$ and $\mathfrak{f}\left(\Omega_{1}\right)$ over $K$. Let $\left\{x_{1}^{*}, \cdots, x_{\omega}^{*}\right\}$ be a regular system of parmaeters in $f(\Omega)$, and let $\left\{y_{1}^{*}, \cdots, y_{\omega_{1}}^{*}\right\}$ be a regular system of parameters in $f\left(\Omega_{1}\right)$. Then the elements $x_{i}^{*}, y_{j}^{*}$, taken together, form a regular system of parameters in $f\left(\Omega \times \Omega_{1}\right)$. If we introduce letters $X_{1}^{*}, \cdots, X_{\omega}{ }^{*}$ and $Y_{1}^{*}, \cdots, Y_{\omega_{1}}{ }^{*}$, then the isomorphisms $J_{x^{*}}$ of $\mathrm{f}(\Omega)$ with $K\left[\left[X^{*}\right]\right]$ and $J_{y^{*}}$ of $\mathrm{f}\left(\Omega_{1}\right)$ with $K\left[\left[Y^{*}\right]\right]$ are the contractions to $f(\Omega)$ and $f\left(\Omega_{1}\right)$ respectively of the isomorphism $J_{x^{*}, y^{*}}$ of $\mathrm{f}\left(\Omega \times \Omega_{1}\right)$ with $K\left[\left[X^{*}, Y^{*}\right]\right]$. We have $J_{x^{*}, y^{*}}(U \times R)=J_{x^{*}}(U) \times J_{y^{*}}(R)$, $J_{x^{*}, y^{*}}(V \times S)=J_{x^{*}}(V) \times J_{y^{*}}(S)$. Therefore, Theorem $4 \mathrm{~b}$ follows immediately from Theorem 4, $\S 4$, p. 39.

A component $M$ of the intersection of three subvarieties $U, V$ and $W$ of $\Omega$ is said to be proper with respect to $\Omega$ if it is of dimension $u+v+w-2 \omega$, where $u, v$ and $w$ are the respective dimensions of $U, V$, and $W$. 
TheOREM 6b. Let $U, V$ and $W$ be three subvarieties of $\Omega$. Assume that $M$ is a proper component of the intersection of $U, V$ and $W$ with respect to $\Omega$. Let $P_{1}, \cdots, P_{0}$ be all the distinct components of the intersection of $U$ and $V$ which contain $M$, and let $Q_{1}, \cdots, Q_{h}$ be all the distinct components of the intersection of $V$ and $W$ which contain $M$. Then $P_{i}$ is a proper component of the intersection of $U$ and $V$ with respect to $\Omega$, and $M$ is a proper component of the intersection of $P_{i}$ and $W$ with respect to $\Omega(1 \leqq i \leqq g) ; Q_{j}$ is a proper component of the intersection of $V$ and $W$ with respect to $\Omega$ and $M$ is a proper component of the intersection of $U$ and $Q_{j}$ with respect to $\Omega$. We have

$$
\sum_{i=1}^{o} i_{\Omega}\left(P_{i} ; U \cdot V\right) i_{\Omega}\left(M ; P_{i} \cdot W\right)=\sum_{j=1}^{h} i_{\Omega}\left(Q_{j} ; V \cdot W\right) i_{\Omega}\left(M ; Q_{j} \cdot U\right) .
$$

This follows immediately from Theorem $6, \S 6$, p. 45 , if we remember that the number $i\left(J_{x^{*}}(M) ; J_{x^{*}}(U) \cdot J_{x^{*}}(V) \cdot J_{x^{*}}(W)\right)$ does not change if we permute $U, V, W$ in any way.

\section{PART III}

1. Algebraic varieties. Let $K$ be an algebraically closed field. To every system of $n$ letters $\left\{X_{1}, \cdots, X_{n}\right\}$ we assign an object $A^{n}(X)$, which we call the affine space with coordinates $X_{1}, \cdots, X_{n}$. To every prime ideal $\mathfrak{u}$ in $K[X]$, we assign (in a one-to-one way) an object $U$ which we call an algebraic variety in $A^{n}(X)$. We say that the variety $U$ and the prime ideal $\mathfrak{u}$ correspond to each other. In particular, we identify $A^{n}(X)$ with the variety which corresponds to the zero ideal.

If $U$ is the algebraic variety which corresponds to the prime ideal $\mathfrak{u}$, the ring $K[X] / \mathfrak{u}$ is called the ring of polynomial functions on $U$; this ring will be denoted by $\mathfrak{f}(U)$. The residue class modulo $\mathfrak{u}$ of a polynomial $F \in K[X]$ is called the function induced by $F$ on $U$. The field of quotients of $f(U)$ is called the field of rational functions on $U$. This field is denoted by $\mathrm{P}(U)$. The ring of quotients of $\mathfrak{u}$ with respect to $K[X]$ is called the neighborhood ring of $U$; this ring is denoted by $\mathfrak{N}(U)$ and its completion by $\mathfrak{N}(U)$.

Proceeding in the same way as we did for algebroid varieties, we define the relationship of inclusion of an algebraic variety $U$ in an algebraic variety $V$, and the related notions, such as the relative neighborhood ring $\mathfrak{N}_{V}(U)$ of $U$ with respect to $V$.

If $U$ is an algebraic variety, the field $\mathrm{P}(U)$ is a finite extension field of $K$. The degree of transcendancy of this extension is called the dimension of $U$. In particular, the dimension of $A^{n}(X)$ is $n$.

Proposition 1. Let $U$ be a variety of dimension $u$ in $A^{n}(X)$. Then $\mathrm{f}(U)$ contains $u$ elements $y_{1}, \cdots, y_{u}$ which are algebraically independent over $K$ and such that $\mathrm{f}(U)$ is finite over $K\left[y_{1}, \cdots, y_{u}\right]$.

Let $\left\{Y_{1}, \cdots, Y_{n}\right\}$ be a system of integrity in $K[X]$ which contains an 
integrity set $\left\{Y_{u^{\prime}+1}, \cdots, Y_{n}\right\}$ of the prime ideal $\mathfrak{u}$ which defines $U$ (cf. Lemma 2, $\$ 1$, part I, p. 5). Then $\mathfrak{u} \cap K[Y]=\mathfrak{u}^{\prime}$ is the ideal generated by $Y_{u^{\prime}+1}, \cdots, Y_{n}$ in $K[Y] ; \mathfrak{f}(U)$ is integral over $K[Y] / \mathfrak{u}^{\prime}$, which is isomorphic with $K\left[Y_{1}, \cdots, Y_{u^{\prime}}\right]$. It follows immediately that $u=u^{\prime}$.

Proposition 2. Let $U$ and $V$ be algebraic varieties in $A^{n}(X)$ such that $U \subset V$. If $u$ and $v$ are the respective dimensions of $U$ and $V$, we have $u \leqq v$ and $\mathfrak{N}_{V}(U)$ is a geometric local ring of dimension $v-u$. The equality $u=v$ implies $U=V$.

Using the notation of the proof of Proposition 1, we observe that the ring of quotients of $\mathfrak{u}^{\prime}$ with respect to $K[Y]$ is a nucleus for $\mathfrak{N}(U)$ (Lemma $7, \S 1$, p. 8). It follows that $\mathfrak{N}(U)$ is a local ring of dimension $n-u$. Let $\mathfrak{v}$ be the prime ideal which corresponds to $V$. Then $\mathfrak{N}(V)$ is isomorphic with $(\mathfrak{N}(U))_{\mathfrak{v}(U)}$; since $\mathfrak{R}(V)$ is of dimension $n-v$, it follows from Theorem 2, $\S 1$, p. 11, that $\mathfrak{v} \mathfrak{N}(U)$ is an ideal of dimension $(n-u)-(n-v)=v-u$ in $\mathfrak{N}(U)$. Therefore $\mathfrak{R}_{V}(U)$, which is isomorphic with $\mathfrak{R}(U) / \mathfrak{v} \mathfrak{N}(U)$, is of dimension $v-u$. If $v=u$, we have $\mathfrak{b} \mathfrak{R}(U)=\mathfrak{u} \mathfrak{R}(U)$, whence $\mathfrak{b}=\mathfrak{u}, V=U$.

The definitions relative to products of algebraic varieties are entirely similar to the corresponding definitions for algebroid varieties and need not be stated here. We can also extend without difficulty the notion of a copy of an affine space and the related notions (diagonal, construction of $M^{\Delta}$, and so on).

An algebraic variety of dimension 0 is called a point. If $p$ is the prime ideal which corresponds to a point $P$, each $X_{k}$ is congruent modulo $\mathfrak{p}$ to an element $a_{k}$ of $K$. The elements $a_{1}, \cdots, a_{n}$ are called the coordinates of $P$.

If a point $P$ is a subvariety of a variety $U$, we say that $P$ lies on $U$, or that $P$ is a point of $U$, or that $U$ goes through $P$.

If $P$ is a variety which corresponds to a maximal prime ideal $\mathfrak{p}$ in $K[X]$ then $P$ is a point. In fact, if $P$ were of dimension $u>0, f(P)$ would be finite over a ring isomorphic with $K\left[X_{1}, \cdots, X_{u}\right]$; making use of Lemma 4, L.R., $\S I I$, p. 694, we see that $f(P)$ would contain ideals distinct from the zero ideal and from $\mathrm{f}(P)$, which is impossible. Since every prime ideal in $K[X]$ is contained in some maximal prime ideal, we see that every algebraic variety con-. tains at least one point.

Proposition 3. Let $V$ be an algebraic variety, and let $U_{1}, \cdots ; U_{a}$ be subvarieties of $V$, all different fron $V$ itself. Then $V$ contains a point which does not lie on any one of the $U_{i}$ 's.

Let $\mathfrak{v}$ and $\mathfrak{u}_{i}$ be the prime ideals which correspond to $V$ and $U_{i}$ respectively. Let $\left\{Y_{1}, \cdots, Y_{n}\right\}$ be a system of integrity in $K[X]$ which contains a set of integrity $\left\{Y_{v+1}, \cdots, Y_{n}\right\}$ of $\mathfrak{b}$. Then $K[X] / \mathfrak{u}_{i}$ is finite over $K[Y] /\left(\mathfrak{u}_{i} \cap K[Y]\right)$ and therefore $\mathrm{P}\left(U_{i}\right)$ is an algebraic extension of the field of quotients of $K[Y] /\left(\mathfrak{u}_{i} \cap K[Y]\right)$. Since $\operatorname{dim} U_{i}<\operatorname{dim} V$, we see that $K[Y] \cap \mathfrak{u}_{i} \neq K[Y] \cap \mathfrak{v}$. It follows that there exists a polynomial $F_{i}\left(Y_{1}, \cdots, Y_{v}\right) \neq 0$ in $Y_{1}, \cdots, Y_{v}$ alone which belongs to $\mathfrak{u}_{i}$. Set $F=\prod_{i=1}^{0} F_{i}$ 
-1 . Then $F$ is not a unit in $K\left[Y_{1}, \cdots, Y_{v}\right]$. It follows immediately that the ideal generated by $\mathfrak{b} \cap K[Y]$ and $F$ in $K[Y]$ is not the unit ideal and is therefore contained in some maximal prime ideal $\mathfrak{p}$ of $K[X]$. The point $P$ which is defined by $\mathfrak{p}$ satisfies our requirements.

2. Sheets of a variety at a point. Let $P$ be a point of coordinates $a_{1}, \cdots, a_{n}$ in the affine space $A^{n}(X)$. Then $\mathfrak{N}(P)$ is the ring of quotients with respect to $K[X]$ of the ideal generated in this ring by $X_{1}-a_{1}, \cdots, X_{n}-a_{n}$, and the completion $\overline{\mathfrak{N}}(P)$ of $\mathfrak{N}(P)$ is $K\left[\left[X_{1}-a_{1}, \cdots, X_{n}-a_{n}\right]\right]$.

Let us introduce $n$ new letters $\bar{X}_{1}, \cdots, \bar{X}_{n}$. Associated to these letters there is a local space $E^{n}(\bar{X})$ (with the same groundfield $K$ as $A^{n}(X)$ ). On the other hand, there exists a uniquely determined isomorphism $J_{P}$ of $\mathfrak{N}(P)$ into $K[[\bar{X}]]$ which maps $X_{k}$ upon $\bar{X}_{k}+a_{k}(1 \leqq k \leqq n)$. The composite object formed by $E^{n}(\bar{X})$ and $J_{P}$ is what we shall call the local space attached to the point $P$ of $A^{n}(X)$. This object will be denoted by $E_{P}^{n}(X)$.

Definition 1. Let $U$ be an algebraic variety in $A^{n}(X)$, and let $\mathfrak{u}$ be the prime ideal which corresponds to $U$. Let $P$ be a point of $U$. Let $\overline{\mathfrak{u}}_{1}, \cdots, \overline{\mathfrak{u}}_{o}$ be the prime divisors of $J_{P}(\mathfrak{u}) K[[\bar{X}]]$. The algebroid varieties in $E_{P}^{n}(\bar{X})$ which correspond to these prime ideals are called the sheets of $U$ at the point $P$.

PROPOSITION 1. If $P$ is a point of the algebraic variety $U$, every sheet of the variety $U$ at $P$ has the same dimension as $U$ itself.

This follows immediately from Theorem 4, $\$ 4$, part I, p. 22 if we observe that $\mathfrak{u} \mathfrak{N}(P)$ is of dimension $u$ in $\mathfrak{R}(P)$ if $U$ is of dimension $u$ (cf. Proposition 2, $\S 1$, p. 58).

Moreover, it follows from Theorem 1, §1, part I, p. 11 that $J_{P}(\mathfrak{u}) K[[\bar{X}]]$ is the intersection of the ideals $\overline{\mathfrak{u}}_{i}(1 \leqq i \leqq g)$ and it follows from Theorem 4 , $\S 4$, part I, p. 22 that $\overline{\mathfrak{u}}_{i} \cap J_{P}(\mathfrak{N}(P))=J_{P}(\mathfrak{u} \mathfrak{N}(P))$. Since $\mathfrak{R}_{U}(P)$ is isomorphic in a natural way with $\mathfrak{R}(P) / \mathfrak{u} \mathfrak{R}(P)$, our last result shows that there is a natural isomorphism of $\mathfrak{N}_{U}(P)$ with a subring of $\mathfrak{N}\left(\bar{U}_{i}\right)$, where $\bar{U}_{i}$ is the sheet which corresponds to $\overline{\mathfrak{H}}_{i}$. This last isomorphism will also be denoted by $J_{P}$. If $F \in K[X], J_{P}$ maps the function induced by $F$ on $U$ upon the function induced by $J_{P}(F)$ on $\bar{U}_{i}$.

Proposition 2. Assume that $U$ is a subvariety of a variety $V$ in $A^{n}(X)$, and let $P$ be a point of $U$. Then every sheet of $U$ at $P$ is contained in at least. one sheet $V$ at $P$.

Using the same notation as above, let furthermore $\mathfrak{b}$ be the prime ideal which corresponds to $V$. Then $\mathfrak{b} \subset \mathfrak{u}$ and therefore $J_{P}(\mathfrak{b}) \subset \overline{\mathfrak{u}}_{i}$. Since $\overline{\mathfrak{u}}_{i}$ is prime, it must contain at least one of the prime ideals of which $J_{P}(\mathfrak{v}) K[[\bar{X}]]$ is the intersection. This observation proves Proposition 2.

Remark. It is not true in general that every sheet of $V$ at $P$ contains some sheet of $U$ at $P$. For instance, the equation $X_{1} X_{2}+X_{1}^{3}+X_{3}^{3}=0$ defines a surface $V$ in $A^{3}\left(X_{1}, X_{2}, X_{3}\right)$; this surface has two sheets at the origin of coordi- 
nates. On the other hand, $V$ contains the line $U$ of equations $X_{1}=X_{3}=0$; this line has one sheet at the origin, and this sheet is contained in only one of the sheets of $V$.

Let us now consider two affine spaces $A^{m}(X)$ and $A^{n}(Y)$. Let $P$ be a point in $A^{m}(X)$ and let $Q$ be a point in $A^{n}(Y)$; then $P \times Q$ (considered as a product of zero-dimensional varieties) is a point in $A^{m}(X) \times A^{n}(Y)$. If we construct $E_{P \times Q}^{m+n}(\bar{X}, \bar{Y})$, we may consider $E^{m+n}(\bar{X}, \bar{Y})$ as the product of $E^{m}(\bar{X})$ and $E^{n}(\bar{Y})$. Moreover, the isomorphism $J_{P \times Q}$ coincides with $J_{P}$ on $\mathfrak{R}(P)$ and with $J_{Q}$ on. $\mathfrak{N}(Q)$ (observe that $\mathfrak{N}(P)$ and $\mathfrak{N}(Q)$ are subrings of $\mathfrak{N}(P \times Q)$ ).

Proposition 3. Let $U$ be an algebraic variety in $A^{m}(X)$, and let $\bar{U}_{1}, \cdots, \bar{U}_{a}$ be the sheets of $U$ at one of its points $P$. Let $V$ be an algebraic variety in $A^{n}(Y)$, and let $\bar{V}_{1}, \cdots, \bar{V}_{b}$ be the sheets of $V$ at one of its points $Q$. Then the sheets of $U \times V$ at $P \times Q$ are the ab algebroid varieties $\bar{U}_{\alpha} \times \bar{V}_{\beta}(1 \leqq \alpha \leqq a, 1 \leqq \beta \leqq b)$.

Let $\mathfrak{u}$ and $\mathfrak{v}$ be the prime ideals in $K[X]$ and $K[Y]$ which correspond to $U$ and $V$ respectively; let $\mathfrak{w}$ be the prime ideal generated by $\mathfrak{u}$ and $\mathfrak{v}$ in $K[X, Y]$, so that $\mathfrak{w}$ is the prime ideal which corresponds to $U \times V$. Let $\overline{\mathfrak{u}}_{\alpha}$ be the prime ideal in $K[[\bar{X}]]$ which corresponds to $\bar{U}_{\alpha}$, and let $\mathfrak{j}_{\beta}$ be the prime ideal in $K[[\bar{Y}]]$ which corresponds to $\bar{V}_{\beta}$. Then the prime ideal in $K[[\bar{X}, \bar{Y}]]$ which corresponds to $\bar{U}_{\alpha} \times \bar{V}_{\beta}$ is the ideal generated by $\overline{\mathfrak{u}}_{\alpha}$ and $\mathfrak{b}_{\beta}$. This ideal contains $J_{P \times Q}(\mathfrak{m})$ and is of dimension $u+v$ if $u$ and $v$ are the dimensions of $U$ and $V$ respectively. It follows immediately that $\bar{U}_{\alpha} \times \bar{V}_{\beta}$ is a sheet of $U \times V$ at $P \times Q$. Conversely, let $\mathfrak{w}$ be a prime ideal in $K[[\bar{X}, \bar{Y}]]$ which contains $J_{P \times Q}(\mathfrak{w})$ and which is of dimension $u+v$. Since $\mathfrak{\mathfrak { w }}$ contains $J_{P}(\mathfrak{u})$, it must contain one of the prime ideals $\overline{\mathfrak{u}}_{\alpha} K[[\bar{X}, \bar{Y}]]$ of which $J_{P}(\mathfrak{u}) K[[\bar{X}, \bar{Y}]]$ is the intersection $\left({ }^{37}\right)$. Similarly, $\mathfrak{\mathfrak { w }}$ must contain one of the ideals $\mathfrak{b}_{\beta} K[[\bar{X}, \bar{Y}]]$. It follows that the sheet $\bar{W}$ of $U \times V$ which corresponds to $\overline{\mathfrak{w}}$ is contained in one of the algebroid varieties $\bar{U}_{\alpha} \times \bar{V}_{\beta}$. Since $\operatorname{dim} \bar{W}=u+v=\operatorname{dim} \bar{U}_{\alpha} \times \bar{V}_{\beta}$, we have $\bar{W}=\bar{U}_{\alpha} \times \bar{V}_{\beta}$. Proposition 3 is thereby proved.

\section{Simple subvarieties.}

Proposition 1. Let $U$ be an algebraic variety in $A^{n}(X)$. Then $\mathfrak{N}(U)$ is a regular local ring. The ring $\overline{\mathfrak{N}}(U)$ contains a subfield isomorphic with $\mathrm{P}(U)$ which is a complete system of representatives for the residue classes modulo the maximal prime ideal in this ring.

Let $x_{1}, \cdots, x_{n}$ be the functions induced by $X_{1}, \cdots, X_{n}$ respectively on $U$. Then $\mathrm{P}(U)=K\left(x_{1}, \cdots, x_{n}\right)$. Since $K$ is algebraically closed, $\mathrm{P}(U)$ is separably generated over $K$, and there exists a subset $\left\{x_{k_{1}}, \cdots, x_{k_{u}}\right\}$ of - $\left\{x_{1}, \cdots, x_{n}\right\}$ which is a separating transcendence base of $\mathrm{P}(U)$ with respect to $K(u$ is the dimension of $U)$. Since $x_{k_{1}}, \cdots, x_{k_{u}}$ are algebraically independent over $K, \mathfrak{N}(U)$ contains the field $K\left(X_{k_{1}}, \cdots, X_{k_{u}}\right)$, and this field

(37) If an element $F(\bar{X}, \bar{Y}) \in K[[\bar{X}, \bar{Y}]]$ belongs to $\bigcap_{\alpha} \mathfrak{u}_{\alpha} K[[\bar{X}, \bar{Y}]]$, the coefficient in $F$ of every monomial in the letters $\bar{Y}$ belongs to $\mathfrak{u}_{\alpha}$ for every $\alpha$, whence $F \in J_{P}(\mathfrak{u}) K[[\bar{X}, \bar{Y}]]$. 
is a basic field of $\mathfrak{R}(U)$. The field of residues of $\mathfrak{N}(U)$, being $\mathrm{P}(U)$, is separable over $K\left(X_{k_{1}}, \cdots, X_{k_{u}}\right)$. It follows from Proposition 3, L.R., §III, p. 701, that $K\left(X_{k_{1}}, \cdots, X_{k_{u}}\right)$ is contained in a subfield $L$ of $\overline{\mathfrak{N}}(U)$ which is a complete set of representatives for the residue classes modulo the maximal prime ideal. Let $\left\{l_{1}, \cdots, l_{n-u}\right\}$ be the complementary set of $\left\{k_{1}, \cdots, k_{u}\right\}$ with respect to $\{1, \cdots, n\}$, and let $\xi_{j}$ be the element of $L$ which belongs to the residue class of $X_{l_{j}}(1 \leqq j \leqq n-u)$. Then $K[X] \subset L\left[X_{l_{1}}-\xi_{1}, \cdots, X_{l_{n-u}}-\xi_{n-u}\right]$. It follows immediately that $X_{l_{1}}-\xi_{1}, \cdots, X_{l_{n-u}}-\xi_{n-u}$ form a set of generators of the maximal prime ideal in $\overline{\mathfrak{N}}(U)$, which proves that $\overline{\mathfrak{N}}(U)$, and therefore also $\mathfrak{N}(U)$, is regular.

Proposition 2. Let $U$ be an algebraic variety of dimension $u$ in $A^{n}(X)$, and let $F_{1}, \cdots, F_{n-u}$ be $n-u$ polynomials which vanish on $U$. Then the following assertions are equivalent: (1) $F_{1}, \cdots, F_{n-u}$ form a regular system of parameters in $\mathfrak{N}(U)$; (2) the Jacobian matrix of $F_{1}, \cdots, F_{n-u}$ does not vanish on $U$.

The proof of Proposition 2 is almost identical with the proof of the corresponding statement for algebroid varieties (Proposition 2, §3, part II, p. 34).

Remark. If we have any number of polynomials which vanish on $U$, then their Jacobian matrix is of rank not greater than $n-u$ on $U$ (cf. the similar remark for algebroid varieties, §3, part II, p. 35).

Definition $\left.1{ }^{38}\right)$. Let $U$ be a subvariety of a variety $V$ in $A^{n}(X)$. If the local ring $\mathfrak{N}_{V}(U)$ is regular, we shall say that $U$ is simple on $V$. If not, we say that $U$ is singular on $V$.

Proposition 3. Let $U$ be a subvariety of an algebraic variety $V$. A necessary and sufficient condition for $U$ to be simple on $V$ is that there should exist a regular system of parameters in $\mathfrak{R}(U)$ which contains a system of parameters in $\mathfrak{N}(V)$.

The proof is identical with the proof of the corresponding assertion for algebroid varieties ( $\$ 3$, part II, p. 35 ).

Proposition 4. Let $U$ be a subvariety of an algebric variety $V$ in $A^{n}(X)$, and let $v$ be the dimension of $V . A$ necessary and sufficient condition for $U$ to be simple on $V$ is that there should exist $n-v$ polynomials which vanish on $V$ and whose Jacobian matrix is of rank $n-v$ on $U$.

(1) Assume that $U$ is simple on $V$. Then there exists a regular system of parameters $\left\{F_{1}, \cdots, F_{n-u}\right\}$ in $\mathfrak{N}(U)$ which contains a system of parameters $\left\{F_{1}, \cdots, F_{n-v}\right\}$ in $\mathfrak{R}(V)$. We may assume without loss of generality that $F_{1}, \cdots, F_{n-u}$ are polynomials. From the fact that the Jacobian matrix of $F_{1}, \cdots, F_{n-u}$ is of rank $n-u$ on $U$, it follows immediately that the Jacobian matrix of $F_{1}, \cdots, F_{n-v}$ is of rank $n-v$ on $U$.

${ }^{(38)}$ This definition of simple subvarieties is due to O. Zariski. Cf. his paper Algebraic varieties over ground fields of characteristic 0, Amer. J. Math. vol. 62 (1940) p. 187. 
(2) Assume that $F_{1}, \cdots, F_{n-v}$ are polynomials which vanish on $V$ and whose Jacobian matrix is of rank $n-v$ on $U$. Let $\left\{G_{1}, \cdots, G_{n-u}\right\}$ be any regular system of parameters in $\mathfrak{N}(U)$. Then we may express $F_{i}$ in the form $F_{i}=\sum_{j=1}^{n-u} A_{i j} G_{j}$ with $A_{i j} \in \mathfrak{R}(U)$. Let $\mathfrak{u}$ be the prime ideal which corresponds to $U$. If we observe that the partial derivatives of a rational function belonging to $\mathfrak{N}(U)$ also belong to $\mathfrak{N}(U)$, we see that $\partial F_{i} / \partial X_{k} \equiv \sum_{j=1}^{n-u} A_{i j} \partial G_{j} / \partial X_{k}$ $(\bmod \mathfrak{u} \mathfrak{N}(U))$. From the fact that the Jacobian matrix of $F_{1}, \cdots, F_{n-v}$ is of rank $n-v$ on $U$, we conclude that one of the determinants of order $n-v$ which can be extracted from the matrix $\left(A_{i j}\right)$ is a unit in $\mathfrak{R}(U)$. If we arrange $G_{1}, \cdots, G_{n-u}$ in a suitable order, we can express $G_{1}, \cdots, G_{n-v}$ as linear combinations of $F_{1}, \cdots, F_{n-v}, G_{n-v+1}, \cdots, G_{n-u}$ with coefficients in $\mathfrak{R}(U)$. It follows that $F_{1}, \cdots, F_{n-v}, G_{n-v+1}, \cdots, G_{n-u}$ form a regular system of parameters in $\mathfrak{R}(U)$, which proves that $U$ is simple on $V$.

Remark. Let there be given a system of generators $\left\{F_{1}, \cdots, F_{h}\right\}$ of the prime ideal $\mathfrak{b}$ which corresponds to $V$. Then, if $U$ is simple on $V$, the $n-v$ polynomials which have the property described in Proposition 4 may be selected among $F_{1}, \cdots, F_{h}$. In fact, every polynomial which vanishes on $V$ is a linear combination of $F_{1}, \cdots, F_{h}$ with coefficients in $K[X]$; it follows that the Jacobian matrix of any system of polynomials which vanish on $V$ is congruent modulo $\mathfrak{v}$ to a multiple of the Jacobian matrix of $F_{1}, \cdots, F_{h}$, and our assertion follows immediately.

Proposition 5. Let $V$ be an algebraic variety in $A^{n}(X)$. Then there exists a finite set $\left\{S_{1}, \cdots, S_{s}\right\}$ (which may be empty) of subvarieties of $V$ with the following properties: (1) each $S_{i}$ is different from $V$; (2) every subvariety of $S_{i}$ is singular on $V$; (3) if a subvariety of $V$ is not contained in any one of the varieties $S_{i}$, then it is simple on $V$.

Let $\left\{F_{1}, \cdots, F_{h}\right\}$ be a set of generators of the prime ideal $\mathfrak{v}$ which corresponds to $V$. Let be the ideal generated by $F_{1}, \cdots, F_{h}$ and by all the determinants of order $n-v$ which can be extracted from the Jacobian matrix of $F_{1}, \cdots, F_{h}$. Let $z_{1}, \cdots, z_{s}$ be the minimal prime divisors of $\&(s=0$ if 8 is the unit ideal), and let $S_{1}, \cdots, S_{s}$ be the subvarieties of $V$ which correspond to $\varepsilon_{1}, \cdots, \varepsilon_{8}$. Since $V$ is clearly regular on itself, we have $z \neq \mathfrak{b}$, whence $S_{i} \neq V$. It follows immediately from Proposition 4 and the remark which follows it that $S_{1}, \cdots, S_{s}$ have the properties (2) and (3).

COROLlaRy 1. If $U$ is a simple subvariety of $V$, then every subvariety of $V$ which contains $U$ is simple.

This follows immediately from Proposition 5.

COROLLARY 2. Let $U$ be a simple subvariety of variety $V$, and let $W_{1}, \cdots, W_{a}$ be subvarieties of $V$ none of which contains $U$. Then there exists a point on $U$ which is simple on both $U$ and $V$ and which does not belong to any one of the varieties $W_{k}$. 
Let the prime ideals $\dot{B}_{i}$ be defined as in the proof of Proposition 5, and let $\mathfrak{w}_{k}$ be the prime ideal which corresponds to $W_{k}$. If $\mathfrak{u}$ is the prime ideal which corresponds to $U$, we denote by $\Xi_{i j}$ the minimal prime divisors (if any) of the ideal generated by $\mathfrak{u}$ and $\mathfrak{s}_{i}$, and by $\mathfrak{w}_{k l}$ the minimal prime divisors (if any) of the ideal generated by $\mathfrak{u}$ and $\mathfrak{w}_{k}$. Let $S_{i j}$ be the subvariety which corresponds to $\varepsilon_{i j}$ and let $W_{k l}$ be the variety which corresponds to $\mathfrak{w}_{k l}$. Then it follows from our assumptions that $S_{i j}$ and $W_{k l}$ are subvarieties of $U$ different from $U$ itself. Let $T_{1}, \cdots, T_{t}$ be the subvarieties of $U$ which play with respect to $U$ the same role as $S_{1}, \cdots, S_{s}$ with respect to $V$. By Proposition 3, $\S 1$, p. 58, $U$ contains a point $P$ which does not belong to any one of the varieties $S_{i j}, W_{k l}, T_{m}$. Such a point has the required properties.

Definition 2. Let $P$ be a simple point on a variety $V$ in $A^{n}(X)$, and let $a_{1}, \cdots, a_{n}$ be the coordinates of $P$. To every polynomial $F$ which vanishes on $V$, let us construct the polynomial $\sum_{k=1}^{n} \partial F / \partial X_{k}\left(a_{1}, \cdots, a_{n}\right) \cdot\left(X_{k}-a_{k}\right)$. Let $\mathrm{t}$ be the prime ideal generated by all linear polynomials obtained in this way. The variety $T$ which corresponds to $t$ is called the tangent linear variety to $V$ at $P$.

It is clear from what was said above that $T$ is of dimension equal to the dimension of $V$ and goes through $P$.

Proposition 6. Let $U$ be a simple subvariety of an algebraic variety $V$, and let $P$ be a point on $U$. Then every sheet $\bar{U}$ of $U$ at $P$ is contained in exactly one sheet $\bar{V}$ of $V$ at $P$, and $\bar{U}$ is simple on $\bar{V}$.

Let $\mathfrak{u}$ and $\mathfrak{v}$ be the prime ideals which correspond to $U$ and $V$ respectively. Denote by $\mathfrak{u}^{*}$ the prime ideal which corresponds to $U$ in $\mathfrak{R}_{V}(P)$ ( $\mathfrak{u}^{*}$ is therefore the ideal generated by $\mathfrak{u} / \mathfrak{b}$ in the ring of quotients of $\mathfrak{p} / \mathfrak{b}$ with respect to $f(V)$, where $p$ is the prime ideal which corresponds to $P$ ). The ring of quotients of $\mathfrak{u}^{*}$ with respect to $\mathfrak{N}_{V}(P)$ is $\mathfrak{R}_{V}(U)$ and is therefore regular. Let $\phi_{1}, \cdots, \phi_{v-u}$ be elements of $\mathfrak{N}_{V}(P)$ which form a regular system of parameters in $\mathfrak{N}_{V}(U)$, whence $e\left(\mathfrak{N}_{V}(U) ; \phi_{1}, \cdots, \phi_{v-u}\right)=1$ by Theorem 3 , §2, part I, p. 14. Let $\overline{\mathfrak{u}}$ be a minimal prime divisor of $\mathfrak{u}^{*} \overline{\mathfrak{N}}_{V}(P)$ in $\overline{\mathfrak{N}}_{V}(P)$ and let $\Phi$ be the natural homomorphism of $\overline{\mathfrak{M}}_{V}(P)$ into $\left(\overline{\mathfrak{N}}_{V}(P)\right)_{\dot{\mathfrak{u}}}$. Then it follows from Theorem 4, $\$ 4$, part I, p. 22 that $e\left(\left(\bar{\Re}_{V}(P)\right)_{\tilde{u}} ; \Phi\left(\phi_{1}\right), \cdots, \Phi\left(\phi_{v-u}\right)\right)=1$. We conclude that $\left(\overline{\mathfrak{R}}_{V}(P)\right)_{\mathfrak{u}}$ is a regular local ring and therefore that the zero ideal in this ring is prime. This means that the kernel of the homomorphism $\Phi$ is a prime ideal in $\overline{\mathfrak{A}}_{V}(P)$ and therefore $\left({ }^{39}\right)$ that $\overline{\mathfrak{u}}$ contains exactly one of the prime divisors of the zero ideal in $\overline{\mathfrak{R}}_{V}(P)$. Now, we have $\overline{\mathfrak{R}}_{V}(P)=\overline{\mathfrak{N}}(P) / \mathrm{v} \overline{\mathfrak{N}}(P)$; it follows that the prime divisors of the zero ideal in $\overline{\mathfrak{R}}_{V}(P)$ correspond in a one-to-one way to the sheets of $V$ at $P$. The minimal prime divisors of $\mathfrak{u}^{*} \overline{\mathfrak{R}}_{V}(P)$ correspond in a one-to-one way to the prime divisors of the zero ideal

$\left.{ }^{(39}\right)$ The kernel of $\phi$ is the intersection of the primary components of the zero ideal in $\overline{\mathfrak{N}}_{V}(P)$ which are contained in $u$; cf. my paper On the ring of quotients of a prime ideal, Bull. Amer. Math. Soc. vol. 50 (1944) p. 93. 
in $\overline{\mathfrak{N}}_{V}(P) / \mathfrak{u} * \overline{\mathfrak{M}}_{V}(P)$, which is a completion of $\mathfrak{\Re}_{V}(P) / \mathfrak{u}^{*} \mathfrak{R}_{V}(P)$ and may therefore be identified with $\overline{\mathfrak{N}}(P) / \mathfrak{u} \overline{\mathfrak{N}}(P)$. It follows that the prime divisors of $\mathfrak{u}^{*} \overline{\mathfrak{A}}_{V}(P)$ correspond in a one-to-one way to the sheets of $U$ at $P$. We have therefore proved that every sheet of $U$ at $P$ is contained in exactly one sheet of $V$ at $P$. Let $\bar{U}$ be the sheet of $U$ which corresponds to $\overline{\mathfrak{u}}$, and let $\bar{V}$ be the sheet of $V$ at $P$ which corresponds to the prime divisor $\mathfrak{v}$ of the zero ideal in $\overline{\mathfrak{N}}_{V}(P)$ which is contained in $\overline{\mathfrak{u}}$, so that $\bar{U} \subset \bar{V}$. Then $\mathrm{f}(\bar{V})$ is isomorphic with $\overline{\mathfrak{R}}_{V}(P) / \mathfrak{b} \overline{\mathfrak{N}}_{V}(P)$ and $\mathfrak{\Re} \bar{V}(\bar{U})$ is isomorphic with the ring of quotients of $\overline{\mathfrak{u}} / \mathfrak{b}$ with respect to $\overline{\mathfrak{R}}_{V}(P) / \overline{\mathfrak{D}} \overline{\mathfrak{R}}_{V}(P)$. But this last ring of quotients coincides by definition with $\left(\overline{\mathfrak{R}}_{V}(P)\right)_{\mathfrak{u}}$, and is therefore regular. It follows that $\bar{U}$ is simple on $\bar{V}$. Proposition 4 is thereby proved.

COROLlary. If $P$ is a simple point on a variety $V$, then $V$ has only one sheet at $P$.

In fact, it is obvious that $P$ has only one sheet at $P$.

Proposition 7. Let $U$ be a subvariety of a variety $V$ in $A^{n}(X)$, and let $P$ be a point of $U$. Assume that there exists a sheet $\bar{U}$ of $U$ at $P$ which is contained in only one sheet $\bar{V}$ of $V$ at $P$ and which is simple on $\bar{V}$. Then $U$ is simple on $V$.

Let $\mathfrak{u}$ and $\mathfrak{v}$ be the prime ideals which correspond to $U$ and $V$; let $\overline{\mathfrak{u}}$ and $\mathfrak{v}$ be the prime ideals in $\bar{\Re}(P)$ which correspond to $\bar{U}$ and $\bar{V}$ respectively. Let $\mathfrak{b}_{1}, \ldots, \mathfrak{b}_{h}$ be the prime ideals in $\overline{\mathfrak{N}}(P)$ of which $\mathfrak{b} \overline{\mathfrak{R}}(P)$ is the intersection (with $\mathfrak{b}_{1}=\mathfrak{v}$ ). Since $\bar{U}$ is simple on $\bar{V}$, we can find $n-v$ power series $\phi_{1}, \cdots, \phi_{n-v}$ in $n$ arguments such that $\phi_{1}(\bar{X}), \cdots, \phi_{n-v}(\bar{X})$ vanish on $\bar{V}$ and that the Jacobian matrix of $\phi_{1}(\bar{X}), \cdots, \phi_{n-v}(\bar{X})$ is of rank $n-v$ on $\bar{U}$. Since $\bar{V}$ is the only sheet of $V$ at $P$ which contains $\bar{U}$, we can find for each $k>1$ an element $\psi_{k} \in \mathfrak{b}_{k}$ which does not belong to $\overline{\mathfrak{u}}$. Set $\psi=\psi_{2} \cdots \psi_{h}$; then $\psi$ can be expressed in the form $\psi\left(X_{1}-a_{1}, \cdots, X_{n}-a_{n}\right)$, where $\psi$ is a power series in $n$ arguments and $a_{1}, \cdots, a_{n}$ are the coordinates of $P$. The Jacobian matrix of $\psi(\bar{X}) \phi_{1}(\bar{X}), \cdots, \psi(\bar{X}) \phi_{n-v}(\bar{X})$ is again of rank $n-v$ on $\bar{U}$. On the other hand, the elements $\psi(X-a) \phi_{i}(X-a)(1 \leqq i \leqq n-v)$ belong to $\bar{v} \overline{\mathfrak{N}}(P)$ and can therefore be written as linear combinations of elements of $\mathfrak{v}$ with coefficients in $K[[X-a]]$. It follows immediately that there exist $n-v$ polynomials in $\mathfrak{v}$ whose images by $J_{P}$ have the property that their Jacobian matrix is of rank $n-v$ on $\bar{U}$. Since $\overline{\mathfrak{u}} \cap K[X]=\mathfrak{u}$, the Jacobian matrix of our $n-v$ polynomials is of rank $n-v$ on $U$, and Propsition 7 is proved.

4. Intersections of algebraic varieties. Let $U$ and $V$ be two algebraic varieties in $A^{n}(X)$. A variety $M$ is said to belong to the intersection of $U$ and $V$ if it is a subvariety of both $U$ and $V$; this being so, if furthermore no variety containing $M$ and different from $M$ belongs to the intersection of $U$ and $V$, then we say that $M$ is a component of the intersection of $U$ and $V$. Exactly as in the case of algebroid varieties, we see that the intersection of $U$ and $V$ has only a finite number of components (this number may here be 0 ) and that 
every variety which is contained in the intersection of $U$ and $V$ is contained in one of the components of this intersection.

Proposition 1. Let $M$ be a component of the intersection of two algebraic varieties $U$ and $V$, and let $P$ be a point of $M$. Let $\bar{U}_{1}, \cdots, \bar{U}_{a}$ be the sheets of $U$ at $P$ and let $\bar{V}_{1}, \cdots, \bar{V}_{b}$ be the sheets of $V$ at $P$. Then every sheet of $M$ at $P$ is a component of the intersection of some $\bar{U}_{\alpha}$ with some $\bar{V}_{\beta}$. Conversely, every component of the intersection of $\bar{U}_{\alpha}$ and $\bar{V}_{\beta}$ is contained in a sheet at $P$ of some component of the intersection of $U$ and $V$.

Let $\mathfrak{u}, \mathfrak{v}$ and $\mathfrak{m}$ be the prime ideals in $K[X]$ which correspond to $U, V$ and $M$ respectively. The sheets $\bar{U}_{\alpha}$ correspond in a one-to-one way to the minimal prime divisors $\overline{\mathfrak{u}}_{\alpha}$ of $\mathfrak{u} \overline{\mathfrak{N}}(P)$ in $\overline{\mathfrak{N}}(P)$; the sheets $\bar{V}_{\beta}$ correspond in a one-to-one way to the minimal prime divisors of the ideal $\mathfrak{v} \overline{\mathfrak{N}}(P)$ in $\overline{\mathfrak{N}}(P)$. Let $\overline{\mathrm{m}}$ be a minimal prime divisor of $\mathfrak{m} \overline{\mathfrak{N}}(P)$. Since $\mathfrak{m}$ contains $\mathfrak{u}$ and $\mathfrak{v}, \overline{\mathfrak{m}}$ contains one of the ideals $\overline{\mathfrak{u}}_{\alpha}$, say $\overline{\mathfrak{u}}_{\alpha_{0}}$, and also one of the prime ideals $\mathfrak{b}_{\beta}$, say $\mathfrak{j}_{\beta_{0}}$. Let $\overline{\mathfrak{m}}^{\prime}$ be a prime ideal contained in $\overline{\mathfrak{m}}$ and containing $\overline{\mathfrak{u}}_{\alpha_{0}}$ and $\mathfrak{v}_{\beta_{0}}$; then $\overline{\mathfrak{m}}^{\prime} \cap K[X]$ is contained in $\mathfrak{m}$ and contains $\mathfrak{u}$ and $\mathfrak{b}$. Since $M$ is a component of the intersection of $U$ and $V$, it follows that $\overline{\mathfrak{m}}^{\prime} \cap K[X]=\mathfrak{m}$, whence $\mathfrak{m} \overline{\mathfrak{R}}(P) \subset \overline{\mathfrak{m}}$. Since $\overline{\mathfrak{m}}$ is a minimal prime divisor of $\mathfrak{m} \overline{\mathfrak{N}}(P)$, it follows that $\overline{\mathfrak{m}}^{\prime}=\overline{\mathfrak{m}}$, which proves that the sheet of $M$ at $P$ which corresponds to $\bar{m}$ is a component of the intersection of $\bar{U}_{\alpha_{0}}$ and $\bar{V}_{\beta_{0}}$.

If $\overline{\mathfrak{n}}$ is any prime ideal in $\overline{\mathfrak{N}}(P)$ which contains $\overline{\mathfrak{u}}_{\alpha}$ and $\mathfrak{j}_{\beta}$, then $\overline{\mathfrak{n}} \cap K[X]$ contains $\mathfrak{u}$ and $\mathfrak{v}$, and therefore also some minimal prime divisor of the ideal generated by $\mathfrak{u}$ and $\mathfrak{v}$ in $K[X]$. Proposition 1 is thereby proved.

Remark. We have proved a little more than we announced, namely that if a sheet $\bar{M}$ of $M$ at $P$ is contained in the intersection of $\bar{U}_{\alpha}$ and $\bar{V}_{\beta}$, then $\bar{M}$ is a component of this intersection.

THEOREM 1. Let $U$ and $V$ be subvarieties of an algebraic variety $\Omega$ in $A^{n}(X)$. Assume that some component $M$ of the intersection of $U$ and $V$ is simple on $\Omega$. Let $u, v$ and $\omega$ be the dimensions of $U, V$ and $\Omega$ respectively. Then the dimension of $M$ is at least $u+v-\omega$.

By Corollary 2 to Proposition $5, \S 3$, p. 62 , we can find a point $P$ on $M$ which is simple on $\Omega$. Then $\Omega$ has only one sheet at $P$ and the origin is simple on this sheet. Theorem 1 follows therefore immediately from Proposition 1 above and from Theorem 1a, §9, part II, p. 53.

Remark 1 . Even in the case where $u+v-\omega \geqq 0$, it cannot be asserted that there exists a variety which belongs to the intersection of $U$ and $V$, as is proved by the example of two generatrices of the same system on a quadric in $A^{3}(X)$.

Remark 2. It follows easily from the previous example that a cone of the second degree in $A^{4}(X)$ will generally contain two linear varieties of dimension 2 which have in common only the vertex of the cone. It follows that the 
assumption that $M$ is simple on $\Omega$ is essential in the statement of Theorem 1 .

Definition 1. Let $U$ and $V$ be two subvarieties of an algebraic variety of dimension $\omega$, and let $u$ and $v$ be the dimensions of $U$ and $V$. A component $M$ of the intersection of $U$ and $V$ is said to be a proper component of this intersection with respect to $\Omega$ if $M$ is simple on $\Omega$ and is of dimension $u+v-\omega$. If $M$ is proper with respect to $A^{n}(X)$, it is said to be a proper component of the intersection of $U$ and $V$.

The reader will observe that, in this last case, the condition about the simplicity of $M$ is automatically satisfied.

We shall now define multiplicities for the proper components of intersections of algebraic varieties.

Definition 2. Let $\Omega$ be an algebraic variety in $A^{n}(X)$, and let $M$ be a subvariety of $\Omega$ which is simple on $\Omega$. Let $\omega$ be the dimension of $\Omega$. Then a set $\left\{X_{i_{1}}, \cdots, X_{i_{\omega}}\right\}$ composed of $\omega$ of the letters $X$ is said to be a set of uniformizing coordinates on $\Omega$ along $M$ if there exists a set of $n-\omega$ polynomials $F_{1}, \cdots, F_{n-\omega}$ which vanish on $\Omega$ and whose functional determinant with respect to the $n-\omega$ letters $X$ which do not occur among $X_{i_{1}}, \cdots, X_{i_{\omega}}$ does not vanish on $M$.

It is clear that such uniformizing coordinates always exist.

Let now $M$ be a component of the intersection of two subvarieties $U$ and $V$ of $\Omega$, and assume that $M$ is proper with respect to $\Omega$. Let $A^{n}\left(X^{\prime}\right)$ be a copy of the space $A^{n}(X)$, and let $V^{\prime}$ be the copy of $V$ in $A^{n}\left(X^{\prime}\right)$. We also construct the diagonal $\Delta$ of $A^{n}(X) \times A^{n}\left(X^{\prime}\right)$ and the subvariety $M^{\Delta}$ of $\Delta$ whose projection on $A^{n}(X)$ is $M$. Let $x_{i}$ and $x_{i}^{\prime}$ be the functions induced on $U \times V^{\prime}$ by $X_{i}$ and $X_{i}^{\prime}$ respectively. Then the $n$ functions $x_{i}^{\prime}-x_{i}$ vanish on $M^{\Delta}$. Conversely, let $N$ be a subvariety of $U \times V^{\prime}$ on which the $n$ functions $x_{i}^{\prime}-x_{i}$ vanish. Then $N$ is contained in $\Delta$ and $\operatorname{pr}_{x} N \subset U, \operatorname{pr}_{x^{\prime}} N \subset V^{\prime}$, whence $\operatorname{pr}_{x} N \subset V$. If $N \supset M^{\Delta}$, we have $M=\operatorname{pr}_{x} N$, whence $M^{\Delta}=N$. It follows that the $n$ elements $x_{i}^{\prime}-x_{i}$ generate an ideal which is primary for the ideal of nonunits in $\mathfrak{R}_{U \times V^{\prime}}\left(M^{\Delta}\right)$. But these elements will not form a system of parameters in $\mathfrak{N}_{U \times V^{\prime}}\left(M^{\Delta}\right)$, because the dimension of $\mathfrak{N}_{U \times V^{\prime}}\left(M^{\Delta}\right)$ is $u+v$ $-(u+v-\omega)=\omega$ and not $n$. Assume now that $X_{i_{1}}, \cdots, X_{i_{\omega}}$ form a system of uniformizing coordinates on $\Omega$ along $M$. Then we shall see that the ideal generated by the $n$ elements $x_{i}^{\prime}-x_{i}(1 \leqq i \leqq n)$ is already generated by the $\omega$ elements $x_{i_{\lambda}}^{\prime}-x_{i_{\lambda}}(1 \leqq \lambda \leqq \omega)$. Let $F_{1}, \cdots, F_{n-\omega}$ be $n-\omega$ polynomials which vanish on $\Omega$ and whose functional determinant with respect to the letters $X$ which do not occur among $X_{i_{1}}, \cdots, X_{i_{\omega}}$ does not vanish on $M$. We have $F_{k}\left(x_{1}, \cdots, x_{n}\right)=0, F_{k}\left(x_{1}^{\prime}, \cdots, x_{n}^{\prime}\right)=0(1 \leqq k \leqq n)$ because $U \times V^{\prime}$ is contained in $\Omega \times \Omega^{\prime}$. Let $\mathfrak{x}$ be the ideal generated by $x_{1}^{\prime}-x_{1}, \cdots, x_{n}{ }^{\prime}-x_{n}$ in $\mathfrak{R}_{U \times V^{\prime}}\left(M^{\Delta}\right)$; then we have by the Taylor expansion theorem

$$
\sum_{j=1}^{n} \partial F_{k} / \partial X_{j}\left(x_{1}, \cdots, x_{n}\right)\left(x_{j}^{\prime}-x_{j}\right) \equiv 0\left(\bmod \mathfrak{x}^{2}\right) .
$$


If $\mathfrak{x}^{\prime}$ is the ideal generated by $x_{i_{1}}^{\prime}-x_{i_{1}}, \cdots, x_{i_{\omega}}^{\prime}-x_{i_{\omega}}$, it follows easily that $\mathfrak{x} \subset \mathfrak{x}^{\prime}+\mathfrak{r}^{2}$. Proceeding by induction on $h$, we conclude that $\mathfrak{x} \subset \mathfrak{x}^{\prime}+\mathfrak{x}^{h}$ for every $h$. Since $\mathfrak{x}^{\prime}$ is closed in the local ring topology of $\mathfrak{R}_{U \times V^{\prime}}\left(M^{\Delta}\right)$, we have $\mathfrak{x}^{\prime}=\mathfrak{x}$, and our assertion is proved. It follows that $x_{i_{1}}^{\prime}-x_{i_{1}}, \cdots, x_{i_{\omega}}^{\prime}-x_{i_{\omega}}$ form a system of parameters in $\mathfrak{R}_{U \times V^{\prime}}\left(M^{\Delta}\right)$.

Definition 3. Let $U$ and $V$ be two subvarieties of an algebraic variety $\Omega$. Assume that $M$ is a proper component of the intersection of $U$ and $V$ with respect to $\Omega$. Let $A^{n}\left(X^{\prime}\right)$ be a copy of the space $A^{n}(X)$ and let $V^{\prime}$ be the copy of $V$ in $A^{n}(X)$. Let $\left\{X_{i_{1}}, \cdots, X_{i_{\omega}}\right\}$ be a system of uniformizing variables on $\Omega$ along $M$, and let $x_{i}$ and $x_{i}^{\prime}$ be the functions induced by $X_{i}$ and $X_{i}^{\prime}$ on $M$. Then the relative multiplicity with respect to $\Omega$ of $M$ in the intersection of $U$ and $V$ is defined to be the number $e\left(\mathfrak{N}_{U \times V^{\prime}}\left(M^{\Delta}\right) ; x_{i_{1}}^{\prime}-x_{i_{1}}, \cdots, x_{i_{\omega}}^{\prime}-x_{i_{\omega}}\right)$. This number is denoted by $i_{\Omega}(M ; U \cdot V)$.

Observe that it follows from what we have proved and from Proposition 3, $\S 2$, part I, p. 14 that the multiplicity $e\left(\mathfrak{N}_{U \times V^{\prime}}\left(M^{\Delta}\right) ; x_{i_{1}}^{\prime}-x_{i_{1}}, \cdots, x_{i_{\omega}}^{\prime}-x_{i_{\omega}}\right)$ does not depend on the choice of the uniformizing coordinates $X_{i_{1}}, \cdots, X_{i_{\omega}}$.

TheOREM 2. Let $U$ and $V$ be subvarieties of an algebraic variety $\Omega$. Assume that $M$ is a proper component of the intersection of $U$ and $V$ with respect to $\Omega$. Let $P$ be a point of $M$, and let $\bar{M}$ be a sheet of $M$ at $P$. Let $\bar{U}_{1}, \cdots, \bar{U}_{a}$ be all the distinct sheets of $U$ at $P$ which contain $\bar{M}$ and let $\bar{V}_{1}, \cdots, \bar{V}_{b}$ be all the distinct sheets of $V$ at $P$ which contain $\bar{M}$; let $\bar{\Omega}$ be the unique sheet of $\Omega$ at $P$. Then $\bar{M}$ is a proper component of the intersection of $\bar{U}_{\alpha}$ and $\bar{V}_{\beta}$ with respect to $\bar{\Omega}$, and we have

$$
i_{\Omega}(M ; U \cdot V)=\sum_{\alpha=1, \beta=1}^{a, b} i \bar{\Omega}\left(\bar{M} ; \bar{U}_{\alpha} \cdot \bar{V}_{\beta}\right) .
$$

Let $u, v$ and $\omega$ be the dimensions of $U, V$ and $\Omega$ respectively. Then $\operatorname{dim} M=\operatorname{dim} \bar{M}=u+v-\omega, \operatorname{dim} \bar{U}_{\alpha}=u, \operatorname{dim} \bar{V}_{\beta}=v$, and the first assertion of Theorem 2 follows immediately from the remark which follows the proof of Proposition 1.

Let $\mathfrak{u}, \mathfrak{v}$ and $\mathfrak{m}$ be the prime ideals in $K[X]$ which correspond to $U, V$ and $M$ respectively. We denote by $P^{\prime}$ the copy of $P$ in $A^{n}\left(X^{\prime}\right)$, by $\mathfrak{v}^{\prime}$ the ideal in $K\left[X^{\prime}\right]$ which corresponds to $V^{\prime}$, by $\mathfrak{w}$ the ideal generated by $\mathfrak{u}$ and $\mathfrak{v}^{\prime}$ in $K\left[X, X^{\prime}\right]$ (that is, also the prime ideal which corresponds to $U \times V^{\prime}$ ), and by $\mathrm{m}^{\Delta}$ the prime ideal in $K\left[X, X^{\prime}\right]$ which corresponds to $M^{\Delta}$. The space $E_{P^{\prime}}^{n}\left(\bar{X}^{\prime}\right)$ may be considered to be a copy of $E_{P}^{n}(\bar{X})$; then the sheets of $V^{\prime}$ at $P^{\prime}$ become the copies of the sheets of $V$ at $P$, and the algebroid varieties in $E_{P}^{n}(\bar{X}) \times E_{P^{\prime}}^{n}\left(\bar{X}^{\prime}\right)$ are put in a one-to-one correspondence with the prime ideals in $\overline{\mathfrak{N}}\left(P \times P^{\prime}\right)$. Let $\overline{\mathfrak{w}}_{\alpha \beta}$ and $\overline{\mathfrak{m}}^{\bar{\Delta}}$ be the prime ideals in $\overline{\mathfrak{N}}\left(P \times P^{\prime}\right)$ which correspond to $\bar{U}_{\alpha} \times \bar{V}_{\beta}^{\prime}$ and to $\bar{M}^{\bar{\Delta}}$ respectively (where $\bar{V}_{\beta}^{\prime}$ is the copy of $\bar{V}_{\beta}$ in $E_{P^{\prime}}^{n}\left(\bar{X}^{\prime}\right)$ and where $\bar{\Delta}$ is the diagonal of $\left.E_{P}^{n}(\bar{X}) \times E_{P^{\prime}}^{n}\left(\bar{X}^{\prime}\right)\right)$.

Let $\left\{X_{i 1}, \cdots, X_{i_{\omega}}\right\}$ be a system of uniformizing coordinates on $\Omega$ at $P$; 
then these letters are also uniformizing coordinates along $M$. Let $x_{i}$ and $x_{i}^{\prime}$ be the functions induced by $X_{i}$ and $X_{i}^{\prime}$ on $U \times V^{\prime}$; then $i_{\Omega}(M ; U \cdot V)$ is the multiplicity of the $\omega$ elements $x_{i_{1}}^{\prime}-x_{i_{1}}, \cdots, x_{i_{\omega}}^{\prime}-x_{i_{\omega}}$, considered as form-

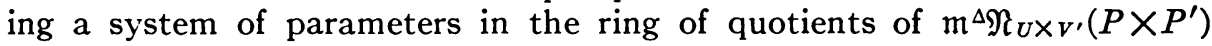
with respect to $\mathfrak{N}_{U \times V^{\prime}}\left(P \times P^{\prime}\right)$. This last ring may be identified with $\mathfrak{R}\left(P \times P^{\prime}\right) / \mathfrak{m} \mathfrak{R}\left(P \times P^{\prime}\right)$, and admits therefore $\overline{\mathfrak{N}}\left(P \times P^{\prime}\right) / \mathfrak{m} \overline{\mathfrak{N}}\left(P \times P^{\prime}\right)$ as a completion. The ideal $\overline{\mathfrak{m}}^{\bar{\Delta}}$ is a minimal prime divisor of $\mathfrak{m}^{\Delta} \overline{\mathfrak{M}}\left(P \times P^{\prime}\right)$; therefore $\overline{\mathfrak{m}}^{\bar{\Delta}} / \mathfrak{w} \overline{\mathfrak{N}}\left(P \times P^{\prime}\right)$ is a minimal prime divisor of $\mathfrak{m}^{\Delta} \overline{\mathfrak{N}}\left(P \times P^{\prime}\right) / \mathfrak{w} \overline{\mathfrak{N}}\left(P \times P^{\prime}\right)$. Let $\phi$ be the natural homomorphism of $\overline{\mathfrak{N}}\left(P \times P^{\prime}\right) / \mathfrak{w} \overline{\mathfrak{N}}\left(P \times P^{\prime}\right)$ into the ring of quotients $\mathfrak{o}$ of $\overline{\mathfrak{m}}^{\bar{\Delta}} / \mathfrak{w} \overline{\mathfrak{N}}\left(P \times P^{\prime}\right)$ with respect to $\overline{\mathfrak{N}}\left(P \times P^{\prime}\right) / \mathfrak{w} \overline{\mathfrak{N}}\left(P \times P^{\prime}\right)$. Then it follows from Theorem 4, $\S 4$, part I, p. 22 that $i_{\Omega}(M ; U \cdot V)$ is equal to $e\left(0 ; \phi\left(x_{i_{1}}^{\prime}-x_{i_{1}}\right), \cdots, \phi\left(x_{i_{\omega}}^{\prime}-x_{i_{\omega}}\right)\right)$. The kernel of $\phi$ is the intersection $\mathfrak{w}^{*}$ of all the prime divisors of the zero ideal in $\overline{\mathfrak{N}}\left(P \times P^{\prime}\right) / \mathfrak{w} \overline{\mathfrak{N}}\left(P \times P^{\prime}\right)$ which are contained in $\overline{\mathfrak{m}}^{\bar{\Delta}} / \mathfrak{w} \overline{\mathfrak{N}}\left(P \times P^{\prime}\right)$; these prime divisors are clearly the ideals $\overline{\mathfrak{w}}_{\alpha \beta} / \mathfrak{w} \overline{\mathfrak{N}}\left(P \times P^{\prime}\right)(1 \leqq \alpha \leqq a, 1 \leqq \beta \leqq b)$. It follows that the prime divisors of the zero ideal in $\mathfrak{D}$ are the ideals $\phi\left(\overline{\mathfrak{w}}_{\alpha \beta} / \mathfrak{w} \overline{\mathfrak{N}}\left(P \times P^{\prime}\right)\right) \mathcal{D}$ and that the zero ideal in $\mathfrak{D}$ is the intersection of these prime ideals. It follows easily from formula (2) $\$ 2$, part I, p. 14, that $e\left(0 ; \phi\left(x_{i_{1}}^{\prime}-x_{i_{1}}\right), \cdots, \phi\left(x_{i_{\omega}}^{\prime}-x_{i_{\omega}}\right)\right)$ is equal to $\sum_{\alpha=1, \beta=1}^{a, b} e\left(\mathfrak{D} / \phi\left(\overline{\mathfrak{w}}_{\alpha \beta} / \mathfrak{w} \overline{\mathfrak{N}}\left(P \times P^{\prime}\right)\right) \mathfrak{D} ; \quad \xi_{i_{1}, \alpha \beta}^{\prime}-\xi_{i_{1}, \alpha \beta}, \cdots, \xi_{i_{\omega}, \alpha \beta}^{\prime}-\xi_{i_{\omega}, \alpha \beta}\right)$, where $\xi_{i, \alpha \beta}$ and $\xi_{i, \alpha \beta}^{\prime}$ are the residue classes of $\phi\left(x_{i}\right)$ and $\phi\left(x_{i}^{\prime}\right)$ modulo $\left(\overline{\mathfrak{w}}_{\alpha \beta} / \mathfrak{w} \overline{\mathfrak{N}}\left(P \times P^{\prime}\right)\right)$ o. Now the ring $\overline{\mathfrak{N}}\left(P \times P^{\prime}\right) / \overline{\mathfrak{w}}_{\alpha \beta} \overline{\mathfrak{N}}\left(P \times P^{\prime}\right)$ is isomorphic with $\mathrm{f}\left(\bar{U}_{\alpha} \times \bar{V}_{\beta}^{\prime}\right)$; the ring $\mathfrak{o} / \phi\left(\overline{\mathfrak{w}}_{\alpha \beta} / \mathfrak{w} \overline{\mathfrak{N}}\left(P \times P^{\prime}\right)\right) \mathrm{o}$ is therefore isomorphic with $\mathfrak{N}_{\overline{\mathfrak{u}}_{\alpha} \times \bar{V}_{\beta}^{\prime}}\left(\bar{M}^{\bar{\Delta}}\right)$ under an isomorphism which maps $\xi_{i, \alpha \beta}$ and $\xi_{i, \alpha \beta}^{\prime}$ upon the functions $\bar{x}_{i}$ and $\bar{x}_{i}^{\prime}$ induced on $\bar{U}_{\alpha} \times \bar{V}_{\beta}^{\prime}$ by $\bar{X}_{i}$ and $\bar{X}_{i}^{\prime}$. It follows that the number $e\left(\mathcal{O} / \phi\left(\overline{\mathfrak{w}}_{\alpha \beta} / \phi \mathfrak{W} \overline{\mathfrak{N}}\left(P \times P^{\prime}\right)\right)_{\mathcal{D}} ; \xi_{i_{1}, \alpha \beta}^{\prime}-\xi_{i_{1}, \alpha \beta}, \cdots, \xi_{i_{\omega}, \alpha \beta}^{\prime}-\xi_{i_{\omega}, \alpha \beta}\right)$ is equal to $\boldsymbol{e}\left(\mathfrak{\Re}_{\overline{\mathfrak{u}}_{\alpha} \times \bar{V}_{\beta}^{\prime}}\left(\bar{M}^{\bar{\Delta}}\right) ; \bar{x}_{i_{1}}^{\prime}-\bar{x}_{i_{1}}, \cdots, \bar{x}_{i_{\omega}}^{\prime}-\bar{x}_{i_{\omega}}\right)$. If $F_{1}, \cdots, F_{n-\omega}$ are $n-\omega$ polynomials which vanish on $\Omega$ and whose functional determinant with respect to the letters $X$ which do not occur among $X_{i_{1}}, \cdots, X_{i_{\omega}}$ does not vanish at $P$, then $F_{1}(\bar{X}), \cdots, F_{n-\omega}(\bar{X})$ vanish on $\bar{\Omega}$ and their functional determinant with respect to the letters $X$ which do not occur among $\bar{X}_{i_{1}}, \cdots, \bar{X}_{i_{\omega}}$ does not vanish at the origin in $E_{P}^{n}(\bar{X})$. It follows that $\bar{x}_{i_{1}}, \cdots, \bar{x}_{i_{\omega}}$ form a regular system of parameters in $\mathrm{f}(\bar{\Omega})$, whence $e\left(\mathfrak{N}_{\overline{\mathfrak{u}}_{\alpha} \times \bar{V}_{\beta}^{\prime}}\left(\bar{M}^{\bar{\Delta}}\right) ; \bar{x}_{i_{1}}^{\prime}-\bar{x}_{i_{1}}, \cdots, \bar{x}_{i_{\omega}}^{\prime}-\bar{x}_{i_{\omega}}\right)$ $=i_{\bar{\Omega}}\left(M ; \bar{U}_{\alpha} \cdot \bar{V}_{\beta}\right)$. Theorem 2 is thereby proved.

Theorem 2 makes it possible to deduce many properties of the multiplicities of intersections of algebraic varieties from the corresponding properties for algebroid varieties.

TheOREM 3. Let $\Omega$ be an algebraic variety in $A^{m}(X)$ and let $U$ and $V$ be two subvarieties of $\Omega$. Let $\Omega_{1}$ be an algebraic variety in $A^{n}(Y)$ and let $R$ and $S$ be two subvarieties of $\Omega_{1}$. Assume that $M$ is a proper component of the intersection of $U$ and $V$ with respect to $\Omega$, and that $N$ is a proper component of the intersection of $R$ and $S$ with respect to $\Omega_{1}$. Then $M \times N$ is a proper component of the intersection of $U \times R$ and $V \times S$ with respect to $\Omega \times \Omega_{1}$, and we have $i_{\Omega \times \Omega_{1}}(M \times N ;(U \times R) \cdot(V \times S))=i_{\Omega}(M ; U \cdot V) i_{\Omega_{1}}(N ; R \cdot S)$. 
It follows immediately from Proposition 4, $\$ 3$, p. 61 that $M \times N$ is simple on $\Omega \times \Omega_{1}$. An easy count of dimensions shows that $M \times N$ is a proper component of the intersection of $U \times R$ and $V \times S$ with respect to $\Omega \times \Omega_{1}$. Let $P$ be a point of $M$ which is simple on $\Omega$; let $P_{1}$ be a point of $N$ which is simple on $\Omega_{1}$. Then $P \times P_{1}$ is simple on $\Omega \times \Omega_{1}$. Let $\bar{M}$ be a sheet of $M$ at $P$ and let $\bar{N}$ be a sheet of $N$ at $P$; then $\bar{M} \times \bar{N}$ is a sheet of $M \times N$ at $P \times P_{1}$ (cf. Proposition 3, §2, p. 60). Let $\bar{U}_{1}, \ldots, \bar{U}_{a}$ be the sheets of $U$ at $P$ which contain $\bar{M}$ and let $\bar{V}_{1}, \cdots, \bar{V}_{b}$ be the sheets of $V$ at $P$ which contain $\bar{M}$; let $\bar{R}_{1}, \cdots, \bar{R}_{c}$ be the sheets of $R$ at $P_{1}$ which contain $\bar{N}$, and let $\bar{S}_{1}, \cdots, \bar{S}_{d}$ be the sheets of $S$ at $P_{1}$ which contain $\bar{N}$. Let $\bar{\Omega}$ be the unique sheet of $\Omega$ at $P$ and let $\bar{\Omega}_{1}$ be the unique sheet of $\Omega_{1}$ at $P_{1}$. Then the algebroid varieties $\bar{U}_{\alpha} \times \bar{R}_{\gamma}(1 \leqq \alpha \leqq a, 1 \leqq \gamma \leqq c)$ are exactly all the sheets of $U \times R$ at $P \times P_{1}$ which contain $\bar{M} \times \bar{N}$ (cf. Proposition 3, \$2, p. 60); the varieties $\bar{V}_{\beta} \times \bar{S}_{\delta}$ are the sheets of $V \times S$ at $P \times P_{1}$ which contain $\bar{M} \times \bar{N}$. It follows from Theorem 2 that $i_{\Omega \times \Omega_{1}}(M \times N ;(U \times R) \cdot(V \times S))=\sum_{\alpha, \beta, \gamma, \delta=1}^{a, b, c, d} i_{\bar{\Omega} \times \bar{\Omega}_{1}}\left(\bar{M} \times \bar{N} ;\left(\bar{U}_{\alpha} \times \bar{R}_{\gamma}\right) \cdot\left(\bar{V}_{\beta} \times \bar{S}_{\delta}\right)\right)$. Making use of Theorem $4 \mathrm{~b}, \S 9$, part II, p. 56 , we see that

$$
i_{\Omega \times \Omega_{1}}\left(\bar{M} \times \bar{N} ;\left(\bar{U}_{\alpha} \times \bar{R}_{\gamma}\right) \cdot\left(\bar{V}_{\beta} \times \bar{S}_{\delta}\right)\right)=i \bar{\Omega}\left(\bar{M} ; \bar{U}_{\alpha} \cdot \bar{V}_{\beta}\right) \cdot i_{\Omega_{1}}\left(\bar{N} ; \bar{R}_{\gamma} \cdot \bar{S}_{\delta}\right),
$$

whence

$$
\begin{aligned}
i_{\Omega+\Omega_{1}}(M \times N ;(U \times R) \cdot(V \times S)) & =\sum_{\alpha, \beta, \gamma, \delta=1}^{a, b, c, d} i \bar{\Omega}\left(\bar{M} ; \bar{U}_{\alpha} \cdot \bar{V}_{\beta}\right) i_{\bar{\Omega}_{1}}\left(\bar{N} ; \bar{R}_{\gamma} \cdot \bar{S}_{\delta}\right) \\
& =i_{\Omega}(M ; U \cdot V) i_{\Omega_{1}}(N ; R \cdot S) .
\end{aligned}
$$

Theorem 3 is thereby proved.

Let now $U, V$ and $W$ be three subvarieties of a variety $\Omega$. Let $u, v$ and $w$ be the respective dimensions of $U, V$ and $W$, and let $\omega$ be the dimension of $\Omega$. A variety $M$ is said to be a component of the intersection of $U, V$ and $W$ if the following conditions are satisfied: $M$ is a subvariety of $U$, of $V$ and of $W$; no variety containing $M$ and different from $M$ itself is contained in $U, V$ and $W$ at the same time. If $M$ is simple on $\Omega$ and is of dimension $u+v+w-2 \omega$, $M$ is said to be a proper component of the intersection of $U, V$ and $W$ with respect to $\Omega$.

THEOREM 4. Let $U, V$ and $W$ be three subvarieties of $a$ variety $\Omega$, and assume that $M$ is a proper component of the intersection of $U, V$ and $W$ with respect to $\Omega$. Let $P_{1}, \cdots, P_{0}$ be the distinct components of the intersection of $U$ and $V$ which contain $M$, and let $Q_{1}, \cdots, Q_{h}$ be the distinct components of the intersection of $V$ and $W$ which contain $M$. Then each $P_{i}$ is a proper component of the intersection of $U$ and $V$ with respect to $\Omega$; each $Q_{j}$ is a proper component of the intersection of $V$ and $W$ with respect to $\Omega ; M$ is a proper component of the intersection of $P_{i}$ and $W$ with respect to $\Omega$ and also a proper component of the intersection of $U$ and $Q_{i}$ with respect to $\Omega$. We have

$$
\sum_{i=1}^{o} i_{\Omega}\left(P_{i} ; U \cdot V\right) i_{\Omega}\left(M ; P_{i} \cdot W\right)=\sum_{j=1}^{h} i_{\Omega}\left(Q_{j} ; V \cdot W\right) i_{\Omega}\left(M ; U \cdot Q_{j}\right) .
$$


Since $P_{i}$ and $Q_{j}$ contain $M$, they are regular on $\Omega$. Let $p_{i}$ be the dimension of $P_{i}$ and let $q_{j}$ be the dimension of $Q_{j}$. We have $p_{i} \geqq u+v-\omega$ and $\operatorname{dim} M \geqq p_{1}+w-\omega$. Since $\operatorname{dim} M=u+v+w-2 \omega$, we have $p_{i}=u+v-\omega$, $\operatorname{dim} M=p_{i}+w-\omega$. It follows that $P_{i}$ is a proper component of the intersection of $U$ and $V$ with respect to $\Omega$ and that $M$ is a proper component of the intersection of $P_{i}$ and $W$ with respect to $\Omega$. A similar argument proves the corresponding assertions for $Q_{j}$. Let $A$ be a point of $M$ which is regular on $\Omega$. We denote by $\bar{M}$ a sheet of $M$ at $A$, and by $\bar{U}_{\alpha}, \bar{V}_{\beta}, \bar{W}_{\gamma}(1 \leqq \alpha \leqq a, 1 \leqq \beta \leqq b$, $1 \leqq \gamma \leqq c)$ those sheets at $A$ of the varieties $U, V, W$ respectively which contain $\bar{M}$. If a sheet of $P_{i}$ at $A$ contains $\bar{M}$, it is a component of the intersection of some $\bar{U}_{\alpha}$ with some $\bar{V}_{\beta}$. Conversely, let $\bar{P}$ be a component of the intersection of $\bar{U}_{\alpha}$ and $\bar{V}_{\beta}$ which contains $\bar{M}$; then $\bar{P}$ is a sheet at $A$ of some component $P$ of the intersection of $U$ and $V$. If $\mathfrak{p}$ is the prime ideal in $\overline{\mathfrak{R}}(A)$ which corresponds to $\bar{P}$, the prime ideal in $K[X]$ which corresponds to $P$ is $\mathfrak{p} \cap K[X]$. Let $\mathfrak{m}$ be the prime ideal in $K[X]$ which corresponds to $M$ and let $\overline{\mathfrak{m}}$ be the prime ideal in $\overline{\mathfrak{N}}(A)$ which corresponds to $\bar{M}$. Then $\mathfrak{m}=\overline{\mathfrak{m}} \cap K[X]$; since $\overline{\mathfrak{p}} \subset \overline{\mathfrak{m}}$, we have $\mathfrak{p} \subset \mathfrak{m}, M \subset P$, which proves that $P$ is one of the components $P_{i}$. This being said, it follows from Theorem 2 and from Theorem $6 \mathrm{~b}$, $\$ 9$, part II, p. 57 that the left side of the formula to be proved is equal to $\sum_{\alpha, \beta, \gamma=1}^{a, b, c} i_{\bar{\Omega}}\left(\bar{M} ; \bar{U}_{\alpha} \cdot \bar{V}_{\beta} \cdot \bar{W}_{\gamma}\right)$, where $\bar{\Omega}$ is the unique sheet of $\Omega$ at $A$. A similar argument shows that the right side is equal to $\sum_{\alpha, \beta, \gamma=1}^{a, b, c} i_{\bar{\Omega}}\left(\bar{M} ; \bar{V}_{\beta} \cdot \bar{W}_{\gamma} \cdot \bar{U}_{\alpha}\right)$. Since $i_{\bar{\Omega}}(\bar{M} ; \bar{U} \cdot \bar{V} \cdot \bar{W})$ is independent of the order in which we take $\bar{U}, \bar{V}, \bar{W}$, Theorem 4 is proved.

Definition 4. Let $U$ and $V$ be two subvarieties of $a$ variety $\Omega$ and let $P$ be a point which belongs to $U$ and $V$. We say that $U$ and $V$ are in general position with respect to each other at $P$ relatively to $\Omega$ if the following conditions are satisfied: (1) $P$ is simple on each one of the varieties $U, V$ and $\Omega$; (2) the intersection of the linear tangent varieties to $U$ and $V$ at $P$ is of dimension $u+v-\omega$, where $u, v$ and $\omega$ are the dimensions of $U, V$ and $\Omega$ respectively. Let $M$ be $a$ common subvariety of $U$ and $V$; then we say that $U$ 'and $V$ are in general position with respect to each other along $M$ relative to $\Omega$ if there exists a point $P$ on $M$ such that $U$ and $V$ are in general position with respect to each other at $P$ relative to $\Omega$.

TheOREM 5. Let $U$ and $V$ be subvarieties of an algebraic variety $\Omega$. Assume that there exists a point $P$ which lies on $U$ and $V$ and is such that $U$ and $V$ are in general position with respect to each other at $P$ relative to $\Omega$. Then $P$ belongs to one and only one component $M$ of the intersection of $U$ and $V$ and $P$ is simple on $M$. The variety $M$ is a proper component of the intersection of $U$ and $V$ with respect to $\Omega$, and we have $i_{\Omega}(M ; U \cdot V)=1$. Conversely, assume that $N$ is a proper component of the intersection of $U$ and $V$ with respect to $\Omega$ and that $i_{\Omega}\left(N ;{ }^{\prime} U \cdot V\right)=1$. Then $U$ and $V$ are in general position with respect to each other along $N$ relative to $\Omega$. 
Assume that $U$ and $V$ are in general position with respect to each other at $P$ relative to $\Omega$. Let $u$ and $v$ be the dimensions of $U$ and $V$, and let $n$ be the dimension of the affine space in which $\Omega$ is a variety. It follows immediately from the definitions that we can find $n-u$ polynomials $F_{1}, \cdots, F_{n-u}$ which vanish on $U$ and $n-v$ polynomials $G_{1}, \cdots, G_{n-v}$ which vanish on $V$ such that the Jacobian matrix of $F_{1}, \cdots, F_{n-u}, G_{1}, \ldots, G_{n-v}$ is of rank $n+\omega-u-v$ at $P$ (where $\omega$ is the dimension of $\Omega$ ). Let $\bar{U}$ be the unique sheet of $U$ at $P$ and let $\bar{V}$ be the unique sheet of $V$ at $P$; then the polynomials $F_{1}(\bar{X}), \cdots, F_{n-u}(\bar{X})$ vanish on $U$ and the polynomials $G_{1}(\bar{X}), \cdots, G_{n-v}(\bar{X})$ vanish on $\bar{V}$ (the letters $\bar{X}$ are the coordinates in the local space attached to $P$ ). It follows from Theorem $9, \S 9$, part II, p. 55 , that the intersection of $\bar{U}$ and $\bar{V}$ has only one component $\bar{M}$, that $\bar{M}$ is a proper component of the intersection of $\bar{U}$ and $\bar{V}$ with respect to $\bar{\Omega}$ (where $\bar{\Omega}$ is the sheet of $\Omega$ at $P$ ), that $i_{\bar{\Omega}}(\bar{M} ; \bar{U} \cdot \bar{V})=1$ and that the origin is simple on $\bar{M}$. Since every component of the intersection of $U$ and $V$ which contains $P$ is of dimension not less than $u+v-\omega$, it follows from Proposition 1 that $P$ belongs to exactly one component $M$ of the intersection of $U$ and $V$, and that $M$ is proper with respect to $\Omega$. It follows from Theorem 2 that $i_{\Omega}(M ; U \cdot V)=1$. It follows from Proposition 7, 33 , p. 64 that $P$ is simple on $M$. The first part of Theorem 5 is thereby proved.

In order to prove the converse part, select a point $P$ on $N$ which is simple on $N$ and $\Omega$ and which does not belong to any component different from $N$ of the intersection of $U$ and $V$ (cf. Corollary 2 to Proposition 5, $\$ 3$, p. 62). Let $\bar{N}$ be the unique sheet of $N$ at $P$; it follows immediately from Theorem 2 that $\bar{N}$ is contained in only one sheet $\bar{U}$ of $U$ at $P$ and in only one sheet $\bar{V}$ of $V$ at $P$ and that $i_{\bar{\Omega}}(\bar{N} ; \bar{U} \cdot \bar{V})=1$, where $\bar{\Omega}$ is the unique sheet of $\Omega$ at $P$. Moreover, the origin is simple on $\bar{N}$ (corollary to Proposition $6, \S 3$, p. 64). We assert that $\bar{U}$ is the only sheet of $U$ at $P$. In fact, assume for a moment that $U$ has another sheet $\bar{U}^{\prime}$ at $P$. Then the intersection of $\bar{U}^{\prime}$ and $\bar{V}$ has at least a component $\bar{N}^{\prime}$ and $\bar{N}^{\prime}$ is different from $\bar{N}$. By Proposition $1, \bar{N}^{\prime}$ is contained in a sheet at $P$ of some component of the intersection of $U$ and $V$; in virtue of our choice of $P$, we have $\bar{N}^{\prime} \subset \bar{N}$, which is impossible since $\bar{N}^{\prime}$ is of dimension not less than $u+v-\omega$ (Theorem 1a, $\S 9$, part II, p. 53) and is different from $\bar{N}$. In the same way we see that $\bar{V}$ is the only sheet of $V$ at $P$. Let $\mathfrak{u}$ and $\mathfrak{v}$ be the prime ideals which correspond to $U$ and $V$ in $K[X]$; let $\overrightarrow{\mathfrak{u}}$ and $\mathfrak{b}$ be the prime ideals in $\overline{\mathfrak{N}}(P)$ which correspond to $\bar{U}$ and $\bar{V}$. Then $\overline{\mathfrak{u}}=\mathfrak{u} \overline{\mathfrak{N}}(P), \mathfrak{v}=\mathfrak{v} \overline{\mathfrak{N}}(P)$. It follows from Theorem $9, \S 9$, part II, p. 55 that we can find $n+\omega-u-v$ power series $\phi_{1}, \cdots, \phi_{n+\omega-u-v}$ in $n$ arguments with the following properties: if $a_{1}, \cdots, a_{n}$ are the coordinates of $P$, each $\phi_{i}\left(X_{1}-a_{1}, \cdots, X_{n}-a_{n}\right)$ belongs either to $\overline{\mathfrak{u}}$ or to $\mathfrak{j}$; the Jacobian matrix of $\phi_{1}(X), \cdots, \phi_{n+\omega-u-v}(X)$ is of rank $n+\omega-u-v$ at the origin in $E_{P}^{n}(\bar{X})$. If $\phi_{i}(X-a)$ belongs to $\overline{\mathfrak{u}}$, it can be written as a linear combination of elements of $\mathfrak{u}$ with coefficients in $K[[X-a]]$; if it belongs to $\mathfrak{b}$, it can be written as a linear combination of 
elements of $\mathfrak{v}$ with coefficients in $K[[X-a]]$. It follows immediately that we can find $n+\omega-u-v$ polynomials $H_{1}, \cdots, H_{n+\omega-u-v}$ of which each belongs either to $\mathfrak{u}$ or to $\mathfrak{v}$ and which are such that their Jacobian matrix is of rank $n+\omega-u-v$ at $P$. It follows also from Theorem 9 , $\$$, part II, p. 55 that the origin is simple on $\bar{U}$ and on $\bar{V}$; by Proposition 7, $\S 3$, p. 64, $P$ is simple on $U$ and on $V$. The intersection of the tangent linear varieties to $U$ and $V$ at $P$ cannot be of dimension greater than $n+\omega-u-v$ in virtue of the existence of the polynomials $H_{i}(1 \leqq i \leqq n+\omega-u-v)$. Since these tangent varieties are of respective dimensions $u$ and $v$ and are both contained in the tangent variety to $\Omega$ at $P$ which is of dimension $\omega$, their intersection is of dimension not less than $n+\omega-u-v$. Theorem 5 is thereby proved.

If $M$ is a proper component of the intersection of two varieties $U$ and $V$ in $A^{n}(X)$, the number $i_{A^{n}(X)}(M ; U \cdot V)$ is denoted by $i(M ; U \cdot V)$. Such intersection multiplicities are said to be absolute (as opposed to relative intersection multiplicities on a variety $\Omega$ ). The following theorem permits to reduce the theory of relative intersections to the theory of absolute intersection multiplicities.

THEOREM 6. Let $\Omega$ be a variety in $A^{n}(X)$ and let $U$ be a simple subvariety of $\Omega$. Let $P$ be a point on $U$ which is simple on $\Omega$. Then there exists a variety $W$ in $A^{n}(X)$ which has the following properties: $U$ is a proper component of the intersection of $W$ and $\Omega$ is the only component of this intersection to contain $P$; we have $i(U ; W \cdot \Omega)=1$. Assume that $M$ is a proper component with respect to $\Omega$ of the intersection of $U$ and of some other variety $V$ in $\Omega$. Let $W$ be a variety which has the properties prescribed above at some point $P$ of $M$ which is simple on $\Omega$. Then $M$ is a proper component of the intersection of $W$ and $V$, and $i_{\Omega}(M ; U \cdot V)=i(M ; W \cdot V)$.

Let $X_{i_{1}}, \cdots, X_{i_{\omega}}$ be uniformizing coordinates at $P$ on $\Omega$ (cf. Definition 2 above). If $\mathfrak{u}$ is the prime ideal which corresponds to $U$, we set $\mathfrak{u}^{\prime}=\mathfrak{u} \cap K\left[X_{i_{1}}, \cdots, X_{i_{\omega}}\right]$, and we denote by $U^{\prime}$ the variety in $A^{\omega}\left(X_{i_{1}}, \cdots\right.$, $\left.X_{i_{\omega}}\right)$ which corresponds to $\mathfrak{u}^{\prime}$. We may represent $A^{n}(X)$ in the form $A^{\omega}\left(X_{i_{1}}, \cdots, X_{i_{\omega}}\right) \times A^{n-\omega}\left(X_{j_{1}}, \cdots, X_{j_{n-\omega}}\right)$, where $X_{j_{1}}, \cdots, X_{j_{n-\omega}}$ are the letters $X$ which do not occur among $X_{i_{1}}, \cdots, X_{i_{\omega}}$. We set $W=U^{\prime}$ $\times A^{n-\omega}\left(X_{j_{1}}, \cdots, X_{j_{n-\omega}}\right)$. Then $W$ clearly contains $U$.

Let $G_{1}, \cdots, G_{n-\omega}$ be $n-\omega$ polynomials which vanish on $\Omega$ and whose functional determinant $D$ with respect to $X_{j_{1}}, \cdots, X_{j_{n-\omega}}$ does not vanish at $P$. Let $u^{\prime}$ be the dimension of $U^{\prime}$, and let $F_{1}, \cdots, F_{\omega-u^{\prime}}$ be $\omega-u^{\prime}$ polynomials in the letters $X_{i_{1}}, \cdots, X_{i_{\omega}}$ whose Jacobian matrix is of rank $\omega-u^{\prime}$ on $U^{\prime}$. We can find a functional determinant $D^{\prime}$ of $F_{1}, \cdots, F_{\omega-u^{\prime}}$ with respect to $\omega-u^{\prime}$ of the letters $X_{i_{1}}, \cdots, X_{i_{\omega}}$ which does not vanish on $U^{\prime}$. Then $D D^{\prime}$ does not vanish on $U$; we can therefore find a point $Q$ on $U$ at which $D D^{\prime} \neq 0$. Then the Jacobian matrix of $F_{1}, \cdots, F_{\omega-u^{\prime}}, G_{1}, \cdots, G_{n-\omega}$ is of rank $n-u^{\prime}$ at $Q$. It follows that the varieties $\Omega$ and $W$ are in general 
position with respect to each other at $Q$. By Theorem 5, p. 70, $Q$ belongs to one and only one component $U_{1}$ of the intersection of $W$ and $\Omega, U_{1}$ is a proper component of this intersection and $i\left(U_{1} ; W \cdot \Omega\right)=1$. Since $U$ is contained in the intersection of $W$ and $\Omega$, we have $U \subset U_{1}$. The dimension of $W$ is $u^{\prime}+n-\omega$, whence $\operatorname{dim} U_{1}=u^{\prime}$. But $U^{\prime}$ is the projection of $U$ on $A^{\omega}\left(X_{i_{1}}, \cdots, X_{i_{\omega}}\right)$, whence $u^{\prime} \leqq u$. It follows that $u^{\prime}=u$ and $U_{1}=U$. The first part of Theorem 6 is proved.

To prove the second part, let $\bar{U}_{\alpha}(1 \leqq \alpha \leqq a)$ and $\bar{V}_{\beta}(1 \leqq \beta \leqq b)$ be the sheets of $U$ and $V$ respectively at $P$; let $\bar{\Omega}$ be the sheet of $\Omega$ at $P$. Since $i(U ; W \cdot \Omega)=1, U$ is simple on $W$. It follows that $\bar{U}_{\alpha}$ is contained in a uniquely determined sheet $\bar{W}_{\alpha}$ of $W$ at $P$ (Proposition $6, \S 3$, p. 63). Since $i(U ; W \cdot \Omega)=1, \bar{W}_{\alpha}$ is the only sheet of $W$ at $P$ to contain $\bar{U}_{\alpha}$ and $i\left(\bar{U}_{\alpha} ; \bar{W}_{\alpha} \cdot \bar{\Omega}\right)$ $=1$. Any component of the intersection of $\bar{W}_{\alpha}$ and $\bar{\Omega}$ is contained in some $\bar{U}_{\alpha^{\prime}}$ (Proposition 1, p. 65) and is of dimension not less than $(n+u-\omega)+\omega-n$ $=u=\operatorname{dim} \bar{U}_{\alpha^{\prime}}$ (Theorem 1, part III, p. 65); it follows that $\alpha^{\prime}=\alpha$ and that $\bar{U}_{\alpha}=\bar{W}_{\alpha} \cdot \bar{\Omega}$. Let $\bar{M}$ be a sheet of $M$ at $P$; we may assume without loss of generality that $\bar{U}_{1}, \cdots, \bar{U}_{a^{\prime}}$ are all the sheets of $U$ at $P$ which contain $\bar{M}$ and that $\bar{V}_{1}, \cdots, \bar{V}_{b^{\prime}}$ are all the sheets of $V$ which contain $\bar{M}$ (where $a^{\prime} \leqq a$, $\left.b^{\prime} \leqq b\right)$. If $\alpha \leqq a^{\prime}, \beta \leqq b^{\prime}, \bar{M}$ is a proper component of the intersection of $\bar{W}_{\alpha}$ and $\bar{V}_{\beta}$ and $i_{\bar{\Omega}}\left(\bar{M} ; \bar{U}_{\alpha} \cdot \bar{V}_{\beta}\right)=i\left(\bar{M} ; \bar{W}_{\alpha} \cdot \bar{V}_{\beta}\right)$ (by Theorem 8 , $\S 9$, part II, p. 54). Any component of the intersection of $W$ and $V$ which contains $M$ is contained in $\Omega$ (because $V \subset \Omega$ ) and is contained in $U$ (because $U$ is the only component of the intersection of $W$ and $\Omega$ which contains $P$ ). Since $M$ is a component of the intersection of $U$ and $V$, it is also a component of the intersection of $W$ and $V$. Since $\bar{M}$ is a proper component of the intersection of $\bar{W}_{\alpha}$ and $\bar{V}_{\beta}, M$ is a proper component of the intersection of $W$ and $V$. If $\alpha$ is an index such that $\bar{M} \subset \bar{W}_{\alpha}$, the intersection of $\bar{W}_{\alpha}$ and $\bar{\Omega}$ is a sheet of $U$ at $P$ which contains $\bar{M}$, whence $\alpha \leqq a^{\prime}$. Therefore, we have by Theorem 2 , p. $67, i(M ; W \cdot V)=\sum_{\alpha, \beta=1,1}^{a^{\prime}, b^{\prime}} i\left(\bar{M} ; \bar{W}_{\alpha} \cdot \bar{V}_{\beta}\right)=\sum_{\alpha, \beta=1,1}^{a^{\prime}, b^{\prime}} i \bar{\Omega}\left(\bar{M} ; \bar{U}_{\alpha} \cdot \bar{V}_{\beta}\right)$ $=i_{\Omega}(M ; U \cdot V)$. Theorem 6 is now completely proved.

Corollary. Let $M$ be a subvariety of a variety $\Omega$, and let $W$ be a variety containing $M$ and such that those components $U_{1}, \cdots, U_{h}$ of the intersection of $W$ and $\Omega$ which contain $M$ are all proper. Let $V$ be a subvariety of $\Omega$ which contains $M$, and assume that $M$ is a proper component with respect to $\Omega$ of the intersection of $U_{i}$ and $V$ for $1 \leqq i \leqq h$. Then $M$ is a proper component of the intersection of $V$ and $W$, and $i(M ; V \cdot W)=\sum_{i=1}^{h} i_{\Omega}\left(M ; U_{i} \cdot V\right)$.

Let $P$ be a point of $M$ which is simple on $\Omega$, and let $T$ be a variety which satisfies the following conditions: $V$ is a proper component of the intersection of $T$ and $\Omega$ and is the only component of this intersection to contain $P$; we have $i(V ; T \cdot \Omega)=1$. Then Theorem 6 says that $M$ is a proper component of the intersection of $T$ and $U_{i}$ and that $i_{\Omega}\left(M ; U_{i} \cdot V\right)=i\left(M ; U_{i} \cdot T\right)$. Let $v, w$ and $\omega$ be the respective dimensions of $V, W$ and $\Omega$. Then each $U_{i}$ is of dimen- 
sion $w+\omega-n, M$ is of dimension $v+w-n$ and $T$ is of dimension $n+v-\omega$. It follows that $M$ is a proper component of the intersection of $W, T$ and $\Omega$. The corollary then follows immediately from Theorem 4, p. 69.

5. Birational invariance of intersection multiplicities. We consider two affine spaces $A^{m}(X)$ and $A^{n}(Y)$. Let $\Omega$ be a variety in $A^{m}(X)$ and let $\Omega_{1}$ be a variety in $A^{n}(Y)$. The varieties $\Omega$ and $\Omega_{1}$ are said to be birationally equivalent when the fields $\mathrm{P}(\Omega)$ and $\mathrm{P}\left(\Omega_{1}\right)$ are isomorphic under an isomorphism which maps every element of $K$ upon itself. An isomorphism of $\mathrm{P}(\Omega)$ with $\mathrm{P}\left(\Omega_{1}\right)$ which maps every element of $K$ upon itself is said to be a birational correspondence between $\Omega$ and $\Omega_{1}$.

We shall assume that there exists a birational correspondence $T$ between $\Omega$ and $\Omega_{1}$. Let $U$ be a subvariety of $\Omega$. We shall say that a subvariety $U_{1}$ of $\Omega_{1}$ corresponds regularly to $U$ by $T$ if $T$ maps $\mathfrak{N}_{\Omega}(U)$ onto $\mathfrak{N}_{\Omega_{1}}\left(U_{1}\right)$. If such is the case, $U_{1}$ is clearly uniquely determined; we shall write $U_{1}=T(U)$. The following assertion follows immediately from the definition: assume that $U_{1}$ corresponds regularly to $U$; let $V$ be a subvariety of $\Omega$ which contains $U$; then there exists a subvariety $V_{1}$ of $\Omega_{1}$ which corresponds regularly to $V$ and $V_{1}$ contains $U_{1}$.

THEOREM 7. Assume that $T$ is a birational correspondence of a variety $\Omega$ with a variety $\Omega_{1}$. Assume that $M$ is a proper component with respect to $\Omega$ of the intersection of two subvarieties $U$ and $V$ of $\Omega$, and that $\Omega_{1}$ contains a variety $M_{1}$ which corresponds regularly to $M$ by $T$. Then $M_{1}$ is a proper component with respect to $\Omega_{1}$ of the intersection of $T(U)$ and $T(V)$, and we have $i_{\Omega_{1}}\left(M_{1} ; T(U) \cdot T(V)\right)=i_{\Omega}(M ; U \cdot V)$.

It is clear that $\operatorname{dim} \Omega=\operatorname{dim} \Omega_{1}, \operatorname{dim} T(U)=\operatorname{dim} U$ and $\operatorname{dim} T(V)=\operatorname{dim} V$. Since $\mathfrak{R}_{\Omega_{1}}\left(M_{1}\right)$ is isomorphic with $\mathfrak{N}_{\Omega}(M)$ and $M$ is simple on $\Omega, M_{1}$ is simple on $\Omega_{1}$. It follows that $M_{1}$ is a proper component of the intersection of $T(U)$ and $T(V)$ with respect to $\Omega_{1}$.

Let $x_{1}, \cdots, x_{m}$ be the functions induced by $X_{1}, \cdots, X_{m}$ on $\Omega$; let $y_{1}, \cdots, y_{n}$ be the functions induced by $Y_{1}, \cdots, Y_{n}$ on $\Omega_{1}$. Then $T\left(x_{i}\right)$ belongs to $\mathfrak{N}_{\Omega_{1}}\left(M_{1}\right)$ and may therefore be written in the form

$$
T\left(x_{i}\right)=P_{i}(y) / Q(y)
$$

where $P_{1}, \cdots, P_{m}$ and $Q$ are polynomials in $n$ arguments and $Q(y)$ does not vanish on $M_{1}$. We can find a point $P_{1}$ on $M_{1}$ which is simple on $\Omega_{1}$ and is such that $Q(y)$ does not vanish at this point. Let $b_{1}, \cdots, b_{n}$ be the coordinates of $P_{1}$; it is clear that the elements $P_{i}(b) / Q(b)(1 \leqq i \leqq m)$ are the coordinates of a point $P$ on $M$ and that $P_{1}$ corresponds regularly to $P$. It follows in particular that $P$ is simple on $\Omega$. Denote by $\bar{\Omega}$ the sheet of $\bar{\Omega}$ at $P$ and by $\bar{\Omega}_{1}$ the sheet of $\Omega_{1}$ at $P_{1}$. The contraction of $T$ to $\mathfrak{N}_{\Omega}(P)$ can be extended to an isomorphism (also denoted by $T$ ) of $\overline{\mathfrak{R}}_{\Omega}(P)$ with $\overline{\mathfrak{R}}_{\Omega_{1}}\left(P_{1}\right)$. It follows that we have an isomorphism (again denoted by $T$ ) of $\mathfrak{f}(\bar{\Omega})$ with $f\left(\bar{\Omega}_{1}\right)$. It is clear that, under the isomorphism $T$, the sheets of $U, V$, and $M$ at $P$ 
correspond to the sheets of $T(U), T(V)$ and $M_{1}$ respectively at $P_{1}$. Theorem 7 then follows immediately from Theorem $2, \S 4$, p. 67 and from Lemma 1, $\$ 9$, part II, p. 52.

6. The projection formula for algebraic varieties. We consider two series of letters $\left\{X_{1}, \cdots, X_{m}\right\}$ and $\left\{Y_{1}, \cdots, Y_{n}\right\}$. Let $U$ be a variety in $A^{m+n}(X, Y)$; then, we may identify $\mathrm{f}^{\mathrm{n}}\left(\operatorname{pr}_{X} U\right)$ with the subring of $\mathrm{f}(U)$ which is generated by the functions induced by $X_{1}, \cdots, X_{m}$ on $U$. It follows that $\mathrm{P}\left(\operatorname{pr}_{x} U\right)$ is a subfield of $\mathrm{P}(U)$ and therefore that $\operatorname{dim}_{i} i_{x} U \leqq \operatorname{dim} U$.

Definition 1. Let $U$ be a variety in $A^{m+n}(X, Y)$. If the dimension of $\operatorname{pr}_{X} U$ is equal to the dimension of $U$, we say that $U$ has a finite projection index on $A^{m}(X)$. In that case, $\mathrm{P}(U)$ may be considered as a finite algebraic extension field of $\mathrm{P}\left(\operatorname{pr}_{x} U\right)$. The degree of this extension is called the projection index of $U$ on $A^{m}(X)$. This number is denoted by $j(U ; X)$.

The reader will have observed that this definition is not a straightforward generalization of the definition used in the case of algebroid varieties, since we do not require here that $f(U)$ be finite over $f\left(\operatorname{pr}_{x} U\right)$. As a consequence, it will not be true in general that every subvariety of a variety which has a finite projection index also has a finite projection index.

Assume that the variety $U$ has a finite projection index on $A^{m}(X)$. We shall denote by $W$ the variety $\operatorname{pr}_{x} U$. Let $P$ be a point of $W$ such that the elements of $\mathfrak{f}(U)$ are integral over the ring $\mathfrak{N}_{W}(P)$ (observe that $\mathfrak{N}_{W}(P)$ is contained in $\mathrm{P}(W)$ which is a subfield of $\mathrm{P}(U))$. Let $P_{1}, \cdots, P_{r}$ be the distinct points of $U$ whose projection on $W$ is $P$ (it is easy to see a priori that $r \geqq 1$; this will also follow from what we shall prove). Let $\bar{X}_{1}, \cdots, \bar{X}_{m}$ be the coordinates in $E_{P}^{m}(X)$; let $\bar{Y}_{1}, \cdots, \bar{Y}_{n}$ be $n$ new letters. We shall use the letters $\bar{X}_{1}, \cdots, \bar{X}_{m}, \bar{Y}_{1}, \cdots, \bar{Y}_{n}$ as coordinates in each of the local spaces attached to $A^{m+n}(X, Y)$. It follows that the sheets of $U$ at all points $P_{i}$ are algebroid varieties in the same local space, which is a product of two local spaces $E^{m}(\bar{X})$ and $E^{n}(\bar{Y})$, of which the first contains the sheets of $W$ at $P$.

Proposition 1. Let $U$ be a variety in $A^{m+n}(X, Y)$ which has a finite projection index on $A^{m}(X)$. Set $W=\operatorname{pr}_{x} U$ and let $P$ be a point of $W$ such that the elements of $\mathrm{f}(U)$ are integral over $\mathfrak{N}_{P}(W)$. Let $P_{1}, \cdots, P_{r}$ be the distinct points of $U$ whose projection on $A^{m}(X)$ is $P$. If $\bar{U}$ is any sheet of $U$ at any one of the points $P_{i}$, then $\operatorname{pr}_{x} \bar{U}$ is a sheet of $W$ at $P$ and $\bar{U}$ has a finite projection index on $E^{m}(\bar{X})$. Let $\bar{W}$ be a given sheet of $W$ at $P$, and let $\bar{U}_{i j}\left(1 \leqq j \leqq s_{i}\right)$ be the distinct sheets of $U$ at $P_{i}$ such that $\operatorname{pr}_{X} \bar{U}_{i j}=\bar{W}(1 \leqq i \leqq r)$. Then we have

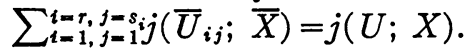

Remark. The sheets $\bar{U}_{i j}$ are not necessarily distinct: although $\bar{U}_{i j} \neq \bar{U}_{i j^{\prime}}$ for $j \neq j^{\prime}$, it may happen that $\bar{U}_{i j}=\bar{U}_{i^{\prime} j^{\prime}}$ for $i^{\prime} \neq i$. This will happen if the variety $U$ is transformed into itself by a translation in $A^{m+n}(X, Y)$ which brings $P_{\boldsymbol{i}}$ onto $P_{\boldsymbol{i}^{\prime}}$. 
Let $\Im$ be the subring of $\mathrm{P}(U)$ which is generated by $\Re_{W}(P)$ and $\mathfrak{f}(U)$. Then $\mathfrak{Y}$ is finite over $\mathfrak{N}_{W}(P)$ and is therefore a semi-local ring (Proposition 3, L.R., §II, p. 694). The maximal prime ideals of $\Im$ are clearly the ideals $p_{1} \Im, \cdots, p_{r} \Im$, where $p_{1}, \cdots, p_{r}$ are the prime ideals in $f(U)$ which correspond to the points $P_{1}, \cdots, P_{r}$. The ring of quotients of $p_{i} \Im$ with respect to $\Im$ is $\Re_{U}\left(P_{i}\right)$.

Let $\bar{U}$ be a sheet of $U$ at a point $P_{i}$. Then $\bar{U}$ corresponds to some prime divisor $\overline{\mathfrak{u}}$ of the zero ideal in $\overline{\mathfrak{M}}_{U}\left(P_{i}\right)$. Let $\bar{\Im}$ be a completion of $\Im$, and let $\epsilon_{i}$ be the idempotent in $\bar{\Im}$ which corresponds to the maximal prime ideal $\mathfrak{p}_{i} \Im$. Then $\overline{\mathfrak{M}}_{U}\left(P_{i}\right)$ is isomorphic with $\bar{\Im} \epsilon_{i}$; there corresponds to $\overline{\mathfrak{u}}$ a prime ideal in $\bar{\Im} \epsilon_{i}$, which may be written in the form $\overline{\mathfrak{u}}^{*} \epsilon_{i}$, where $\overline{\mathfrak{u}}^{*}$ is a prime ideal containing $1-\epsilon_{i}$ in $\bar{\Im}$.

The adherence of $\mathfrak{N}_{P}(W)$ in $\bar{\Im}$ is a completion $\overline{\mathfrak{N}}_{P}(W)$ of $\mathfrak{N}_{P}(W)$ and $\bar{\Im}$ is finite over $\overline{\mathfrak{N}}_{P}(W)$ (cf. Proposition 7, L.R., §II, p. 699). Denote by $\mathfrak{w}$ the prime ideal $\overline{\mathfrak{u}}^{*} \cap \overline{\mathfrak{M}}_{W}(P)$. The ideal $\overline{\mathfrak{u}}^{*}$ is clearly a divisor of the zero ideal in $\bar{\Im}$; since no nonzero divisor of $\mathfrak{N}_{W}(P)$ becomes a zero divisor in $\bar{\Im}$ (cf. L.R., loc. cit. above), $\mathfrak{w}$ is a prime divisor of the zero ideal in $\overline{\mathfrak{N}}_{W}(P)$ and therefore there corresponds to $\overline{\mathfrak{w}}$ a sheet $\bar{W}$ of $W$ at $P$. The ring $\bar{\Im} / \overline{\mathfrak{u}}^{*}$ is isomorphic with $\mathrm{f}(\bar{U})$ and is finite over $\overline{\mathfrak{N}}_{W}(P) / \overline{\mathfrak{w}}$ which is isomorphic with $\mathrm{f}(\bar{W})$. We conclude easily from this that $\bar{W}$ is the projection of $\bar{U}$ on $E^{m}(X)$ and that $\bar{U}$ has a finite projection index with respect to $E^{n}(\bar{X})$. The first part of Proposition 1 is thereby proved.

Let $\overline{\mathfrak{u}}_{i, j}^{*}$ be the prime ideals in $\bar{\Im}$ which correspond (in the way explained above) to the sheets $\bar{U}_{i, j}\left(1 \leqq j \leqq s_{i}\right)$; these ideals are the prime divisors of the zero ideal in $\bar{\Im}$ which contain $1-\epsilon_{i}$ and $\mathfrak{w}$. Since a prime ideal in $\bar{\Im}$ cannot contain at the same time $1-\epsilon_{i}$ and $1-\epsilon_{i^{\prime}}$ for $i \neq i^{\prime}$, we have $\overline{\mathfrak{u}}_{i^{\prime} j}^{*} \neq \overline{\mathfrak{u}}_{\mathfrak{i j}}^{*}$ for $\left(i^{\prime}, j^{\prime}\right) \neq(i, j)$. Let $Z$ and $Z^{\prime}$ be the rings of quotients of $\overline{\mathfrak{N}}_{W}(P)$ and $\bar{\Im}$ respectively; then $Z$ and $Z^{\prime}$ are hypercomplex systems over suitable fields. If $\left\{u_{1}, \cdots, u_{d}\right\}$ is a maximal system of elements of $\Im$ linearly independent with respect to $\mathfrak{N}_{W}(P)$, then $u_{1}, \cdots, u_{d}$ are also linearly independent over $Z$ in $Z^{\prime}$ and $Z^{\prime}=Z u_{1}+\cdots+Z u_{d}$ (cf. Proposition 7, L.R., §II, p. 699). Since $\mathfrak{\mathfrak { w }}$ is a prime divisor of the zero ideal in $\overline{\mathfrak{N}}_{W}(P)$, there corresponds to $\mathfrak{\mathfrak { w }}$ an idempotent $\eta$ in $Z$ such that $\mathfrak{w}=Z \eta \cap \overline{\mathfrak{N}}_{W}(P)$. The ring $\overline{\mathfrak{N}}_{W}(P)(1-\eta)$ is isomorphic with $f(\bar{W})$ (in fact, the kernel of the mapping $z \rightarrow z(1-\eta)$ of $\overline{\mathfrak{M}}_{W}(P)$ is $\overline{\mathfrak{w}})$. The ring $\bar{\Im}(1-\eta)$ is finite over $\overline{\mathfrak{N}}_{W}(P)(1-\eta)$ and the prime divisors of the zero ideal in this ring are the ideals $\overline{\mathfrak{u}}_{i j}^{*}(1-\eta),\left(1 \leqq i \leqq r, 1 \leqq j \leqq s_{i}\right)$. The ring $\bar{\Im}(1-\eta) / \overline{\mathfrak{u}}_{i j}^{*}(1-\eta)$ is isomorphic with $\bar{\Im}_{\mathfrak{u}} \overline{\mathfrak{u}}_{i j}^{*}$, that is, with $\mathfrak{f}\left(\bar{U}_{i j}\right)$. It follows easily that

$$
\left[\bar{\Im}(1-\eta) / \overline{\mathfrak{u}}_{i j}^{*}(1-\eta): \overline{\mathfrak{M}}_{W}(P)(1-\eta)\right]=j\left(\bar{U}_{i j} ; \bar{X}\right) .
$$

On the other hand, since the $\overline{\mathfrak{u}}_{i j}^{*}(1-\eta)$ are all the prime divisors of the zero ideal in $\bar{\Im}(1-\eta)$, the sum of the numbers $\left[\bar{\Im}(1-\eta) / \overline{\mathfrak{u}}_{i j}^{*}(1-\eta): \overline{\mathfrak{R}}_{W}(P)(1-\eta)\right]$ (for all combinations of indices $i$ and $j$ ) is equal to $\left[Z^{\prime}(1-\eta): Z(1-\eta)\right]$. 
The elements $u_{1}, \cdots, u_{d}$ being linearly independent over $Z$, it follows immediately that $u_{1}(1-\eta), \cdots, u_{d}(1-\eta)$ are linearly independent over $Z(1-\eta)$, whence $\sum_{i, j} j\left(\bar{U}_{i j} ; \bar{X}\right)=d=\left(\Im: \Re_{W}(P)\right)=[\mathrm{P}(U): \mathrm{P}(W)]=j(U ; X)$. Proposition 1 is proved.

Theorem 8. Let $U$ be a variety in $A^{m+n}(X)$ and let $V$ be a variety in $A^{m}(X)$. Assume that $M$ is a proper component of the intersection of $\operatorname{pr}_{x} U$ and $V$ and that the elements of $\mathrm{f}(U)$ are integral over $\mathfrak{N}_{\mathrm{pr}_{X} U}(M)$. Then $U$ has a finite projection index on $A^{m}(X)$. If $M_{1}, \cdots, M_{h}$ are the distinct components of the intersection of $U$ and $V \times A^{n}(Y)$ whose projection on $A^{m}(X)$ is $M$, then each $M_{k}$ is a proper component of the intersection of $U$ and $V \times A^{n}(Y)$ and has a finite projection index on $A^{m}(X)$. We have

$$
j(U ; X) i\left(M ;\left(\operatorname{pr}_{X} U\right) \cdot V\right)=\sum_{k=1}^{h} j\left(M_{k} ; X\right) i\left(M_{k} ; U \cdot\left(V \times A^{n}(Y)\right) .\right.
$$

We set $W=\operatorname{pr}_{x} U$. The field of quotients of $\mathfrak{f}(U)$ is $\mathrm{P}(U)$ and the field of quotients of $\mathfrak{N}_{W}(M)$ is $\mathrm{P}(W)$. It follows immediately that $U$ has a finite projection index on $A^{m}(X)$. To every $M_{k}$ there corresponds a prime ideal $\mathfrak{m}_{k}$ in $\mathfrak{f}(U)$ such that $\mathfrak{m}_{k} \cap \mathfrak{f}(W)=\mathfrak{m}$, where $\mathfrak{m}$ is the prime ideal which corresponds to $M$ in $\mathrm{f}(W)$. We have $\mathfrak{N}_{W}(M) \subset \mathfrak{N}_{U}\left(M_{k}\right)$. Let $\Im$ be the ring generated by $\mathfrak{N}_{W}(M)$ and $\mathfrak{f}(U)$; then $\Im$ is finite over $\mathfrak{N}_{W}(M)$ and $\mathfrak{m}_{k} \Im$ is one of the maximal prime ideals of $\Im$. The field $\Im / \mathfrak{m}_{k} \Im$ may be identified with $\mathrm{P}\left(M_{k}\right)$; the field $\mathfrak{N}_{W}(M) / \mathfrak{m} \mathfrak{N}_{W}(M)$ is $\mathrm{P}(M)$. It follows immediately that $M_{k}$ has a finite projection index on $A^{m}(X)$. In particular, we have $\operatorname{dim} M_{k}=\operatorname{dim} M=u+v-m$ $=u+(v+n)-(m+n)$, if $u$ and $v$ are the dimensions of $U$ and $V$ respectively. It follows that $M_{k}$ is a proper component of the intersection of $U$ and $V \times A^{n}(Y)$.

The functions $y_{1}, \cdots, y_{n}$ induced on $U$ by $Y_{1}, \cdots, Y_{n}$ respectively satisfy equations with coefficients in $\mathfrak{N}_{W}(M)$ and with leading coefficient 1 . These coefficients may be written as fractions whose numerators and denominators belong to $\mathfrak{f}(W)$, the denominator being the same for all these fractions and not belonging to $\mathrm{m}$. It follows that there exists a polynomial $D(X)$ in the letters $X$ which does not vanish on $M$ and which has the following property: if $P$ is a point of $M$ at which $D(X)$ does not vanish, then the elements of $\mathfrak{f}(U)$ are integral over $\mathfrak{N}_{W}(P)$.

The components of the mutual intersections of the varieties $M_{k}(1 \leqq k \leqq h)$ have dimensions less than $u+v-m$, and the same applies to the projections of these components on $A^{m}(X)$. Similarly, the projections on $A^{m}(X)$ of the varieties which are singular on $M_{k}$ are of dimensions less than $u+v-m$. It follows from Proposition 5, $\S 3$, p. 62, and from Corollary 2 to this proposition that we can find a point $P$ on $M$ which satisfies the following conditions: $P$ is simple on $M$; if a point $Q$ belonging to some $M_{k}$ is such that $\operatorname{pr}_{x} Q=P$, then $Q$ belongs to only one of the varieties $M_{k}$ and is simple on $M_{k}$; the ele- 
ments of $\mathfrak{f}(U)$ are integral over $\mathfrak{R}_{W}(P)$. We shall denote by $P_{1}, \cdots, P_{g}$ the distinct points of $U$ whose projection is $P$. Each $P_{i}$ belongs to some $M_{k}$. In fact, $P$ is contained in some component $M^{\prime}$ of the intersection of $U$ and $V \times A^{n}(Y)$, and $\operatorname{dim} M^{\prime} \geqq u+v-m$ by Theorem $1, \S 4$, p. 65 . Since $P$ belongs to $\operatorname{pr}_{X} M^{\prime}$ and since the elements of $\mathrm{f}(U)$ are integral over $\mathfrak{N}_{W}(P), M^{\prime}$ has a finite projection index on $A^{m}(X)$ (cf. the argument used above for $M_{k}$ ). It follows that $\operatorname{dim} \operatorname{pr}_{x} M^{\prime} \geqq u+v-m$. Since $\operatorname{pr}_{x} M^{\prime} \subset M$, we have $\operatorname{pr}_{x} M^{\prime}=M$ and $M^{\prime}=M_{k}$ for some $k$.

By construction, $P_{i}$ belongs to only one of the varieties $M_{k}$. We shall denote by $k_{i}$ the index $k$ such that $P_{i} \subset M_{k}$. We denote by:

$\bar{M}$ the sheet of $M$ at $P$;

$\bar{M}_{k_{i}}$ the sheet of $M_{k_{i}}$ at $P_{i}$;

$\bar{U}_{i j}\left(1 \leqq j \leqq s_{i}\right)$ the sheets of $U$ at $P_{i}$;

$\bar{W}_{\alpha}(1 \leqq \alpha \leqq a)$ the sheets of $W$ at $P$;

$\bar{V}_{\beta}(1 \leqq \beta \leqq b)$ the sheets of $V$ at $P$.

We shall evaluate in two different ways the sum

$$
\sum_{i=1}^{g} \sum_{j=1}^{s_{i}} \sum_{\beta=1}^{b} i\left(\bar{M}_{k_{i}} ; \bar{U}_{i j} \cdot\left(\bar{V}_{\beta} \times E^{n}(\bar{Y})\right)\right) j\left(\bar{M}_{k_{i}} ; \bar{X}\right)
$$

(observe that the sheets of $V \times A^{n}(Y)$ at $P_{i}$ are the varieties $\bar{V}_{\beta} \times E^{n}(\bar{Y})$ $(1 \leqq \beta \leqq b)$; in virtue of our assumptions on $P, \bar{M}_{k_{i}}$ is the only component of the intersection of $\bar{U}_{i j}$ and $\bar{V}_{\beta} \times E^{n}(\bar{Y})$ ).

First, if we sum all terms which correspond to a fixed value of $i$, we obtain $i\left(M_{k_{i}} ; U \cdot\left(V \times A^{n}(Y)\right) \cdot j\left(\bar{M}_{k_{i}} ; \bar{X}\right)\right.$ (this, by Theorem $2, \S 4$, p. 67). If we take the sum of all the terms for which $k_{i}$ has a given value $k$, we obtain $i\left(M_{k} ; U \cdot\left(V \times A^{n}(Y)\right) j\left(M_{k} ; X\right)\right.$ (this, by Proposition 1 above). It follows that the sum (1) is equal to $\sum_{k=1}^{n} i\left(M_{k} ; U \cdot\left(V \times A^{n}(Y)\right)\right) j\left(M_{k} ; X\right)$. On the other hand, we have $\operatorname{pr}_{x} \bar{U}_{i j}=\bar{W}_{\alpha(i, j)}$, where $\alpha(i, j)$ is some index between 1 and $a$ which depends on $i$ and $j$; making use of Theorem 5 , $\$ 5$, part II, p. 42, we obtain $i\left(\bar{M}_{k_{i}} ; \bar{U}_{i j} \cdot\left(\bar{V}_{\beta} \times E^{n}(\bar{Y})\right)\right) j\left(\bar{M}_{k_{i}} ; \bar{X}\right)=i\left(\bar{M} ; \bar{W}_{\alpha(i, j)} \cdot \bar{V}_{\beta}\right) \cdot j\left(\bar{U}_{i j} ; \bar{X}\right)$. If we sum over all combinations $(i, j)$ such that $\alpha(i, j)$ has a given value $\alpha$, we obtain by Proposition 1 above $i\left(\bar{M} ; \bar{W}_{\alpha} \cdot \bar{V}_{\beta}\right) \cdot j(U ; X)$. If we then sum over all combinations $(\alpha, \beta)$, we get (by Theorem $2, \S 4$, p. 67) $i(M ; W \cdot V) j(U ; X)$. Theorem 6 is thereby proved.

7. Cycles. In analogy with what we did in the case of algebroid varieties, we introduce the notion of cycle on an algebraic variety.

Definition 1. By a cycle of dimension $u$ in $A^{n}(X)$ we mean a formal linear combination of a finite number of u-dimensional algebraic varieties in $A^{n}(X)$ with integral non-negative coefficients. If all varieties which occur with positive coefficients in the cycle are subvarieties of some variety $\Omega$, we say that we have a cycle on $\Omega$. 
The cycles of a given dimension can be added together and multiplied by non-negative integers. A variety $U$ will be identified with the cycle $1 \cdot U$.

DEFINITION 2. Let $\Omega$ be a variety in $A^{n}(X)$, and let $U$ and $V$ be two subvarieties of $\Omega$. We say that $U$ and $V$ have a relative intersection cycle on $\Omega$ if every component of the intersection of $U$ and $V$ is proper with respect to $\Omega$. This being the case, we set $(U \cdot V)_{\Omega}=\sum_{M} i_{\Omega}(M ; U, V) M$, the sum being extended over all components $M$ of the intersection of $U$ and $V$. Let $\sum_{i} a_{i} U_{i}=X$ and $\sum_{j} b_{j} V_{j}=Y$ be two cycles on $\Omega$, with $U_{i} \neq U_{i^{\prime}}$ for $i \neq i^{\prime}, V_{j} \neq V_{j^{\prime}}$ for $j \neq j^{\prime}$. If whenever $a_{i} b_{j} \neq 0$ the symbol $\left(U_{i} \cdot V_{j}\right)_{\Omega}$ is defined, then we set $(X \cdot Y)_{\Omega}=\sum_{i j} a_{i} b_{j}\left(U_{i} \cdot V_{j}\right)_{\Omega} ;(X \cdot Y)_{\Omega}$ is called the relative intersection cycle of $X$ and $Y$ on $\Omega$. If $X$ and $Y$ are any cycies, we say that $X \cdot Y$ is defined when $(X \cdot Y)_{A^{n}(X)}$ is defined, and then we set $X \cdot Y=(X \cdot Y)_{A^{n}(X)}$.

Let us consider two affine spaces $A^{n_{1}}\left(X^{(1)}\right)$ and $A^{n_{2}}\left(X^{(2)}\right)$. Let $X_{1}=\sum_{i} a_{i} U_{i, 1}$ be a cycle in $A^{n_{1}}\left(X^{(1)}\right)$ and let $X_{2}=\sum_{j} b_{j} U_{j, 2}$ be a cycle in $A^{n_{2}}\left(X^{(2)}\right)$. Then we denote by $X_{1} \times X_{2}$ the cycle $\sum_{i j} a_{i} b_{j} U_{i, 1} \times U_{j, 2}$ in $A^{n_{1}}\left(X^{(1)}\right) \times A^{n_{2}}\left(X^{(2)}\right)$.

Theorem 3a. Let $\Omega_{i}$ be a variety in $A^{n_{i}}\left(X^{(i)}\right)(i=1,2)$, and let $X_{i}$ and $Y_{i}$ be two cycles on $\Omega_{i}$. If the cycles $\left(X_{1} \cdot Y_{1}\right)_{\Omega_{1}}$ and $\left(X_{2} \cdot Y_{2}\right)_{\Omega_{2}}$ are defined, then the cycle $\left(\left(X_{1} \times X_{2}\right) \cdot\left(Y_{1} \times Y_{2}\right)\right)_{\Omega_{1} \times \Omega_{2}}$ is defined and is equal to $\left(\left(X_{1} \cdot Y_{1}\right)_{\Omega_{1}}\right)$ $X\left(\left(X_{2} \cdot Y_{2}\right)_{\Omega_{2}}\right)$.

This follows immediately from Theorem 3, §4, p. 68 .

THEOREM 4a. Let $X, Y$ and $Z$ be three cycles on a variety $\Omega$, all different from 0 . If either one of the cycles $\left((X \cdot Y)_{\Omega} \cdot Z\right)_{\Omega}$ and $\left(X \cdot(Y \cdot Z)_{\Omega}\right)_{\Omega}$ is defined, then both are defined, and they are equal to each other.

This follows easily from Theorem 4, §4, p. 69 (cf. the proof of Theorem 6a, §8, part II, p. 51).

TheOREM 6a. Let $X$ be a cycle and let $\Omega$ be a variety. Let $Y$ be a cycle on $\Omega$. If $((X \cdot \Omega) \cdot Y)_{\Omega}$ is defined, then $(X \cdot Y) \cdot \Omega$ is defined and is equal to $((X \cdot \Omega) \cdot Y)_{\Omega}$.

This follows easily from the corollary to Theorem $6, \S 4$, p. 72.

Let $\left\{X_{1}, \cdots, X_{m}\right\}$ and $\left\{Y_{1}, \cdots, Y_{n}\right\}$ be two series of letters. If a variety $U$ in $A^{m+n}(X, Y)$ has a finite projection index on $A^{m}(X)$, we say that the cycle al.pr. $X U$ is defined and we set al.pr. $X U=j(U ; X) U$. If $X=\sum_{i} a_{i} U_{i}$ is a cycle in $A^{m+n}(X, Y)$, and if al.pr. $x U_{i}$ is defined whenever $a_{i} \neq 0$, we say that al.pr. $X X$ is defined and equal to $\sum_{i} a_{i}$ al.pr.x $U_{i}$.

Theorem 7a. Let $X$ be a cycle in $A^{m+n}(X, Y)$ and let $Y$ be a cycle in $A^{m}(X)$. Assume that the cycle (al.pr. $x X) \cdot Y$ is defined. Then al.pr.x $\left(X \cdot\left(Y \times A^{n}(Y)\right)\right)$ is defined and equal to (al.pr. $x X) \cdot Y$.

This follows immediately from Theorem $8, \S 6$, p. 77. 
8. Intersections with hypersurfaces. A variety of dimension $n-1$ in $A^{n}(X)$ is called a hypersurface. The following facts are well known (and can be established in the same way as the corresponding results for algebroid hypersurfaces): the prime ideal which corresponds to a hypersurface is a principal ideal, generated by an irreducible polynomial; conversely, every irreducible polynomial generates a prime ideal which corresponds to a hypersurface. If $F$ is an irreducible polynomial, we shall denote by $(F)$ the hypersurface which corresponds to the ideal generated by $F$, and we shall say that $F=0$ is an equation of this hypersurface.

If $F$ is any polynomial not equal to 0 , we set $(F)=\left(F_{1}\right)+\cdots+\left(F_{h}\right)$ if $F=a F_{1} \cdots F_{h}$ is the decomposition of $F$ in the product of a unit $a$ and of some irreducible polynomials $F_{1}, \cdots, F_{h}$ (we set $(F)=0$ if $F$ is a unit).

Proposition 1. Let $U$ be a variety in $A^{n}(X)$ and let $(F)$ be an $(n-1)$ dimensional cycle such that $U \cdot(F)$ is defined. Then, if $M$ is a variety which occurs with a coefficient not equal to 0 in $U \cdot(F)$, the function $F^{U}$ induced by $F$ on $U$ is a parameter in $\mathfrak{N}_{U}(M)$, and the coefficient with which $M$ occurs in $U \cdot(F)$ is equal to $e\left(\mathfrak{R}_{U}(M) ; F^{U}\right)$. If $M$ is a simple subvariety of $U$, there exists exactly one valuation $v$ in $\mathfrak{N}_{U}(M)$ whose domain of values is the set of all nonnegative integers, and $e\left(\mathfrak{R}_{U}(M) ; F^{U}\right)=v\left(F^{U}\right)$.

Let $P$ be a simple point on $M$, and let $\bar{M}$ be the sheet of $M$ at $P$. Let $F_{1}, \cdots, F_{k}$ be the irreducible factors of $F$ which vanish at $P$. If $\bar{X}_{1}, \cdots, \bar{X}_{n}$ are the coordinates in the local space attached to $A^{n}(X)$ at $P$, the polynomial $F_{i}(\bar{X})$ can be decomposed in a product of irreducible power series $F_{i j}(\bar{X})$ in $K[[\bar{X}]]$. Since $\overline{\mathfrak{N}}(P) / F_{i} \overline{\mathfrak{N}}(P)$ is an intersection of prime ideals, we see that the algebroid hypersurfaces $\left(F_{i j}\right)$ are all distinct; they are obviously the sheets at $P$ of the hypersurfaces $\left(F_{i}\right)$. Let $\bar{U}_{1}, \cdots, \bar{U}_{\alpha}$ be the sheets of $U$ at $P$. Then the coefficient of $M$ in $U \cdot(F)$ is clearly equal to the sum $\sum_{\alpha, i, j} i\left(\bar{M} ; \bar{U}_{\alpha}\right.$ $\cdot\left(F_{i j}(\bar{X})\right)$. This is equal to the sum of the coefficients of $\bar{M}$ in the cycles $\bar{U}_{\alpha} \cdot(F(\bar{X}))$, that is, to $\sum_{\alpha} e\left(\Re_{\bar{U}_{\alpha}}(M) ; F^{\bar{U}_{\alpha}}(\bar{X})\right)$, where $F^{\bar{U}_{\alpha}}(\bar{X})$ is the function induced on $\bar{U}_{\alpha}$ by $F(\bar{X})$ (cf. Proposition 2, §7, part II, p. 48). Let $\mathfrak{m}$ and $\mathfrak{u}$ be the prime ideals which correspond to $M$ and $U$ respectively in $K[X]$; then $\mathfrak{m} \overline{\mathfrak{N}}(P)=\overline{\mathfrak{m}}$ is the prime ideal which corresponds to $\bar{M}$ in $\overline{\mathfrak{N}}(P)$, and $\mathfrak{u} \overline{\mathfrak{N}}(P)$ is the intersection of the prime ideals $\overline{\mathfrak{u}}_{\alpha}$ which correspond to the varieties $\bar{U}_{\alpha}$. The ring $\overline{\mathfrak{N}}(P) / \mathfrak{u} \overline{\mathfrak{N}}(P)$ is a completion $\overline{\mathfrak{N}}_{U}(P)$ of $\mathfrak{N}_{U}(P)$ and the ideals $\overline{\mathfrak{u}}_{\alpha} / \mathfrak{u} \overline{\mathfrak{N}}(P)$ are the prime divisors of the zero ideal in this ring. They are all contained in $\overline{\mathfrak{m}} / \mathfrak{u} \overline{\mathfrak{N}}(P)$. If we denote by $F_{\alpha}^{*}$ the residue class of $F^{\bar{U}_{\alpha}}$ modulo $\overline{\mathfrak{u}}_{\alpha}^{\prime}, e\left(\Re_{\bar{U}_{\alpha}}(\bar{M}) ; F^{\bar{U}_{\alpha}}(\bar{X})\right)$ is equal to the multiplicity of $F^{*}$ considered as a parameter in the ring of quotients of $\overline{\mathfrak{m}} / \overline{\mathfrak{u}}_{\alpha}$ with respect to $\overline{\mathfrak{N}}(P) / \overline{\mathfrak{u}}_{\alpha} \overline{\mathfrak{N}}(P)$. It follows from formula (2), §2, part I, p. 14, that $\sum_{\alpha} e\left(\Re_{\bar{U}_{\alpha}}(\bar{M}) ; F^{\bar{U}_{\alpha}}(\bar{X})\right)$ is the multiplicity of $F^{U}$ considered as a parameter in the ring of quotients of $\overline{\mathfrak{m}} / \mathfrak{u} \overline{\mathfrak{N}}(P)$ with respect to $\overline{\mathfrak{N}}(P) / \mathfrak{u} \overline{\mathfrak{N}}(P)$. By Theorem 4 , 4 , part I, p. 22 
this multiplicity is equal to the multiplicity of $F^{U}$ as a parameter in the ring of quotients $\mathfrak{R}_{U}(M)$ of $\mathfrak{m} / \mathfrak{u} \mathfrak{N}(P)$ with respect to $\mathfrak{N}(P) / \mathfrak{u} \mathfrak{N}(P)$.

Assume that $M$ is simple on $U$. Then $\mathfrak{N}_{U}(M)$ is a regular local ring of dimension 1 , and therefore this ring has exactly one valution $v$ whose domain of values is the set of all non-negative integers and $e\left(\mathfrak{R}_{U}(M) ; F^{U}\right)=a v\left(F^{U}\right)$, where $a$ is factor of proportionality which does not depend upon $F$ (cf. end of $\S 7$, part II, p. 48). Since $e\left(\Re_{U}(M) ; F^{U}\right)$ is always an integer, $a$ is an integer. It follows from Theorem $6, \S 4$, p. 72 that $e\left(\mathfrak{N}_{U}(M) ; F^{U}\right)=1$ for a suitable choice of $F$. Therefore $a=1$, and Proposition 1 is proved.

9. Intersection multiplicities and levels of inseparability. All fields to be considered in this section are supposed to be of characteristic $p \neq 0$.

Proposition 1. Let $Z_{0} / K_{0}$ be a finite extension of a field $K_{0}$, and let $L / K_{0}$ be a finite algebraic purely inseparable extension of $K_{0}$. Then the ratio $\left[L: K_{0}\right] /\left[Z_{0} L: Z_{0}\right]$ is bounded from above by a number which depends on $K_{0}$ and $Z_{0}$ only, not on $L$.

Let $\left\{z_{1}, \cdots, z_{r}\right\}$ be a transcendence base of $Z_{0} / K_{0}$. We have $\left[Z_{0} L: K_{0}(z)\right]$ $=\left[Z_{0} L: L(z)\right]\left[L(z): K_{0}(z)\right]$. The second factor is equal to $\left[L: K_{0}\right]$ because $K_{0}(z)$ is separably generated over $K_{0}$. On the other hand, we may also write $\left[Z_{0} L: K_{0}(z)\right]=\left[Z_{0} L: Z_{0}\right]\left[Z_{0}: K_{0}(z)\right]$. It follows that the ratio under consideration is equal to $\left[Z_{0}: K_{0}(z)\right] /\left[Z_{0} L: L(z)\right]$. The numerator of this last fraction being independent of $L$, Proposition 1 is proved.

Definition 1. The situation being as described in Proposition 1, the maximal value taken by the ratio $\left[L: K_{0}\right] /\left[Z_{0} L: Z_{0}\right]$ is called the level of inseparability of the extension $Z_{0} / K_{0}$.

It is clear that, for any $L$, the ratio under consideration is an integer and a power of $p$. Therefore the level of inseparability is a power of $p$. It follows immediately from the definition that the level of inseparability is 1 if and only if the extension $Z_{0} / K_{0}$ is separably generated( $\left.{ }^{40}\right)$.

Proposition 2. Let $Z_{0} / K_{0}$ be a finite extension of $K_{0}$, and let $L / K_{0}$ be a finite purely inseparable extension of $K_{0}$. A necessary and sufficient condition for the ratio $\left[L: K_{0}\right] /\left[Z_{0} L: Z_{0}\right]$ to be equal to the level of inseparability of $Z_{0} / K_{0}$ is that $L Z_{0}$ be separably generated over $L$.

Let $L_{1} / L$ be any finite purely inseparable extension of $L$. Then we have

$$
\frac{\left[L_{1}: K_{0}\right]}{\left[Z_{0} L_{1}: Z_{0}\right]}=\frac{\left[L_{1}: L\right]}{\left[Z_{0} L_{1}: Z_{0} L\right]} \frac{\left[L: K_{0}\right]}{\left[Z_{0} L: Z_{0}\right]}
$$

and the ratio $\left[L_{1}: L\right] /\left[Z_{0} L_{1}: Z_{0} L\right]$ is integral. If $\left[L: K_{0}\right] /\left[Z_{0} L: Z_{0}\right]$ is equal to

$\left(^{40}\right)$ Cf. my paper Some properties of ideals in rings of power series, Trans. Amer. Math. Soc. vol. 55 (1944) Proposition 2, p. 69. 
the level of inseparability of $Z_{0} / K_{0}$, then $\left[L_{1}: L\right]=\left[Z_{0} L_{1}: Z_{0} L\right]$ for any finite purely inseparable extension $L_{1} / L$ of $L$, which proves that $L Z_{0}$ is separably generated over $L$. Conversely, if this last condition is satisfied, the ratio $\left[L: K_{0}\right] /\left[Z_{0} L: Z_{0}\right]$ does not change if we replace $L$ by $L_{1}$. Let $L^{\prime} / K_{0}$ be a finite purely inseparable extension of $K_{0}$ such that $\left[L^{\prime}: K_{0}\right] /\left[Z_{0} L^{\prime}: Z_{0}\right]$ is equal to the level of inseparability of $Z_{0} / K_{0}$, and set $L_{1}=L_{0} L^{\prime}$. It follows immediately from formula (1) above, applied to $L^{\prime}$ instead of $L$, that $\left[L_{1}: K_{0}\right] /\left[Z_{0} L_{1}: Z_{0}\right]$ $=\left[L^{\prime}: K_{0}\right] /\left[Z_{0} L^{\prime}: Z_{0}\right]$; in virtue of our choice of $L^{\prime}$, the equality sign prevails. Proposition 2 is thereby proved.

Proposition 3. Let $Z_{0} / K_{0}$ be a finite extension of $K_{0}$ and let $K_{1} / K_{0}$ be a separable algebraic extension of $K_{0}$. Then the level of inseparability of $Z_{0} K_{1} / K_{1}$ is equal to the level of inseparability of $Z_{0} / K_{0}$.

Let $L / K_{0}$ represent a purely inseparable extension of $K_{0}$ such that $\left[L: K_{0}\right] /\left[Z_{0} L: Z_{0}\right]$ is equal to the level of inseparability of $Z_{0} / K_{0}$. Since $K_{1}$ is separably generated over $K_{0}$, we have $\left[L K_{1}: K_{1}\right]=\left[L: K_{0}\right]$. Since $Z_{0} K_{1}$ is separably generated over $Z_{0}$, we have $\left[Z_{0} K_{1} L: Z_{0} K_{1}\right]=\left[Z_{0} L: Z_{0}\right]$. Since $Z_{0} L$ is separably generated over $L$ (by Proposition 2), $Z_{0} K_{1} L$ is separably generated over $K_{1} L$. Proposition 3 follows therefore immediately from Proposition 2.

Let now $U$ be a variety in $A^{n}(X)$, defined by a prime ideal $\mathfrak{u}$ in $K[X]$. Let $K_{0}$ be a subfield of $K$; if $x_{1}, \cdots, x_{n}$ are the functions induced on $U$ by $X_{1}, \cdots, X_{n}$, we denote by $K_{0}[U]$ the subring $K_{0}\left[x_{1}, \cdots, x_{n}\right]=K_{0}[x]$ of $\mathrm{f}(U)$, and we denote by $K_{0}(U)$ the field of quotients of $K_{0}[U]$.

Proposition 4. Let $U$ be a variety in $A^{n}(X)$ and let $M$ be a subvariety of $U$. Let $K_{0}$ be a subfield of $K$ in which $U$ is definable, and assume that $K_{0}(U)$ contains a certain number of elements $u_{1}, \cdots, u_{s}$ which form a system of parameters in $\mathfrak{N}_{U}(M)$. Then $M$ is definable in some algebraic extension of $K_{0}$ and $e\left(\mathfrak{N}_{U}(M) ; u_{1}, \cdots, u_{s}\right)$ is divisible by the level of inseparability of the extension $K_{0}(M) / K_{0}$.

Let $K_{0}^{*}$ be the algebraic closure of $K_{0}$. The first part of Proposition 4 will be proved if we show that $M$ is definable in $K_{0}^{*}$. Let $\mathfrak{u}$ and $\mathfrak{m}$ be the prime ideals in $K[X]$ which correspond to $U$ and $M$ respectively. We set $\mathfrak{u}^{*}=\mathfrak{u} \cap K_{0}^{*}[X], \mathfrak{m}^{*}=\mathfrak{m} \cap K_{0}^{*}[X]$. Since $U$ is definable in $K_{0}^{*}$, we have $\mathfrak{u}^{*} K[X]=\mathfrak{u}$. Since $K_{0}^{*}$ is algebraically closed, the ideal $\mathfrak{m}^{\prime}=\mathfrak{m}^{*} K[X]$ is prime, and defines a variety $M^{\prime}$. It is clear that $M^{\prime}$ is a subvariety of $U$ and contains $M$; moreover, the elements $u_{1}, \cdots, u_{s}$ belong to $\mathrm{m}^{\prime} / \mathfrak{u}$. Since these elements form a system of parameters in $\mathfrak{R}_{U}(M)$, it follows immediately that $M^{\prime}=M$, which proves our assertion.

In order to prove the second assertion of Proposition 4, we may without loss of generality replace $K_{0}$ by a field which is algebraic and separable over $K_{0}$ (cf. Proposition 3 above). We may therefore assume that the algebraic closure of $K_{0}$ is purely inseparable over $K_{0}$. 
We can find elements $y_{1}, \cdots, y_{m}$ in $K_{0}[U]$ such that the functions $y_{1}^{\prime}, \cdots, y_{m}^{\prime}$ induced on $M$ by $y_{1}, \cdots, y_{m}$ respectively form a transcendence base of $K_{0}(M) / K_{0}$. These functions are algebraically independent over $K_{0}^{*}$ in $K_{0}^{*}(M)$ and therefore also on $K$ in $K(M)$ (this, because $M$ is definable in $\left.K_{0}^{*}\right)$. It follows that $y_{1}^{\prime}, \cdots, y_{m}^{\prime}$ form a transcendence base of $K(M) / K$ and that $M$ is of dimension $m$. The field $K\left(y_{1}, \cdots, y_{m}\right)=K(y)$ is a basic field of $\mathfrak{R}_{U}(M)$; it follows immediately that $y_{1}, \cdots, y_{m}, u_{1}, \cdots, u_{s}$ are algegebraically independent over $K$. On the other hand, the dimension $s$ of $\mathfrak{R}_{U}(M)$ is equal to the difference between the dimensions of $U$ and $M$; it follows that $\operatorname{dim} U=m+s$, and therefore that $K(U)$ is algebraic over $K(y, u)$.

We introduce a completion $\overline{\mathfrak{N}}_{U}(M)$ of $\mathfrak{N}_{U}(M)$; then $K(y)$ is a basic field of $\overline{\mathfrak{R}}_{U}(M), u_{1}, \cdots, u_{s}$ are analytically independent over $K(y)$ and $\overline{\mathfrak{R}}_{U}(M)$ is finite over $K(y)[[u]]$. Let $\mathfrak{D}$ be the subring of $\overline{\mathfrak{R}}_{U}(M)$ which is generated by $K(y)[[u]]$ and $\mathfrak{N}_{U}(M)$. Then $\mathfrak{D}$ is finite over $K(y)[[u]]$ and is therefore a complete semi-local ring (Proposition 3, L.R., §II, p. 694). The intersections of $\mathfrak{D}$ with the powers of the ideal of nonunits in $\overline{\mathfrak{R}}_{U}(M)$ form a fundamental system of neighbourhoods of 0 in the semi-local ring topology of $\mathfrak{D}$ (this, by Lemma 7, L.R., §II, p. 695). It follows that the identity mapping of $\mathfrak{D}$ into $\overline{\mathfrak{N}}_{U}(M)$ is continuous. Since $\mathfrak{D}$ contains $\mathfrak{R}_{U}(M)$, we have $\mathfrak{D}=\overline{\mathfrak{N}}_{U}(M)$. Let $Z$ be the ring of quotients of $\overline{\mathfrak{N}}_{U}(M)$; then $Z$ contains $K(y)((u)$ ) (cf. Proposition 1, §2, part I, p. 12) and it follows from what we have just proved that $Z$ is generated by adjunction to $K(y)((u))$. of the elements of $K(U)$.

Denote by $Z_{0}$ the subring of $Z$ which is generated by $K_{0}(y)((u))$ and by $K_{0}(U)$. Since $K_{0}(U)$ is algebraic over $K_{0}(y, u), Z_{0}$ may be considered as a hypercomplex system over $K_{0}(y)((u))$. We shall prove that $Z$ coincides with the hypercomplex system $Z^{\prime}$ which is deduced from $Z_{0}$ by extending the field of coefficients from $K_{0}(y)((u))$ to $K(y)((u))$.

First, it is clear that $Z$ is a homomorphic image of $Z^{\prime}$. On the other hand, $Z^{\prime}$ is a homomorphic image of the hypercomplex system $Z^{\prime \prime}$ deduced from $K_{0}(U)$ (considered as a hypercomplex system over $K_{0}(y, u)$ ) by extending the field of coefficients from $K_{0}(y, u)$ to $K(y)((u))$. This extension may be carried out in two steps, first extending to $K(y, u)$ and from there to $K(y)((u))$. Since $U$ is definable over $K_{0}$, the first step leads to $K(U)$. Since $K(y)((u))$ is separably generated over $K(y, u)$, we see that $Z^{\prime \prime}$ is semi-simple. The same holds a fortiori for $Z^{\prime}$. It follows that $Z=Z^{\prime} \epsilon$, where $\epsilon$ is an idempotent in $Z^{\prime}$. Let $\left\{\zeta_{1}, \cdots, \zeta_{h}\right\}$ be a base of $Z_{0} / K_{0}(y)((u))$; we express $\epsilon$ in the form $\sum_{i=1}^{h} \xi_{i} \zeta_{i}$ with $\xi_{1}, \cdots, \xi_{h} \in K(y)((u))$. If we express that $\epsilon$ is an idempotent, we obtain a certain number of algebraic relations with coefficients in $K_{0}(y)((u))$ between the quantities $\xi_{1}, \cdots, \xi_{h}$. These relations, considered as equations in the unknowns $\xi_{1}, \cdots, \xi_{h}$, have only a finite number of solutions in the algebraic closure $\Sigma$ of $K(y)((u))$ (because $\left(Z_{0}\right)_{\Sigma}$ has only a finite number of idempotents). It follows that these solutions are all algebraic over $K_{0}(y)((u))$. Making use of the fact that $K_{0}^{*}$ is purely inseparable over $K_{0}$ 
and of a result proved elsewhere, we conclude that each $\xi_{k}$ is purely inseparable over $\left.K_{0}(y)((u)){ }^{41}\right)$. Therefore, we have $\epsilon=\epsilon^{p^{\nu}} \in Z_{0}$ for some $\nu>0$. Remembering that $Z_{0}$ is a subring of $Z$, we see that the inclusion $\epsilon \in Z_{0}$ implies $\epsilon=1$, whence $Z=Z^{\prime}$.

Let $x_{1}, \cdots, x_{n}$ be the functions induced by $X_{1}, \cdots, X_{n}$ on $U$. Then each $x_{i}$ belongs to $\overline{\mathfrak{N}}_{U}(M)$, and is therefore integral over $K(y)[[u]]$. It follows that the normal equation satisfied by $x_{i}$ (considered as an element of the hypercomplex system $Z$ ) has its coefficients in $K(y)[[u]]$. On the other hand, $x_{i}$ belongs to $Z_{0}$ and therefore the coefficients of the normal equation of $x_{i}$ are in $K_{0}(y)((u))$. It follows that these coefficients are in $K_{0}(y)((u))$ $\cap K(y)[[u]]=K_{0}(y)[[u]]$. This means that each $x_{i}$ is integral over $K_{0}(y)[[u]]$. Let $\mathfrak{D}_{0}$ be the subring of $Z_{0}$ which is generated by $K_{0}(y)[[u]]$ and $x_{1}, \cdots, x_{n}$. The ring $\mathfrak{D}_{0}$ is finite over $K_{0}(y)[[u]]$ and is therefore a complete semi-local ring. Since $\mathfrak{D}_{0} \subset \overline{\mathfrak{N}}_{U}(M)$, $\mathfrak{D}_{0}$ cannot contain any idempotent not equal to 1 ; since $K_{0}(y) \subset \mathfrak{D}_{0}, \mathfrak{D}_{0}$ is a complete local ring. Let $\mathfrak{M}_{0}$ be the maximal prime ideal of $\mathfrak{D}_{0} ; \mathfrak{M}_{0}$ is contained in the ideal of nonunits of $\overline{\mathfrak{N}}_{U}(M)$. Let $\mathfrak{m}_{0}$ be the prime ideal in $K_{0}[U]$ composed of the functions belonging to $K_{0}[U]$ which vanish on $M$. An element of $\mathfrak{m}_{0}$ is a nonunit in $\mathfrak{N}_{U}(M)$ and therefore also in $\mathfrak{D}_{0}$; it follows that $\mathfrak{m}_{0} \subset \mathfrak{M}_{0}$. The ideal $\mathfrak{M}_{0} \cap K_{0}[U]$ is prime, contains $\mathfrak{m}_{0}$, and has only 0 in common with $K_{0}(y)$; it follows easily that $\mathfrak{M}_{0} \cap K_{0}[U]=\mathfrak{m}_{0}$. Let $x_{1}^{\prime}, \cdots, x_{n}^{\prime}$ be the functions induced on $M$ by $x_{1}, \cdots, x_{n}$; since $u_{1}, \cdots, u_{s}$ are in $\mathfrak{M}_{0}$, we have $\mathfrak{D}_{0} / \mathfrak{M}_{0}=K_{0}\left(y^{\prime}\right)\left[x_{1}^{\prime}, \cdots\right.$, $\left.x_{n}^{\prime}\right]=K_{0}(M)$.

The ring $\mathfrak{D}_{0}$ is clearly equidimensional. It follows that $\left[Z_{0}: K_{0}(y)((u))\right]$ $=e\left(\mathfrak{D}_{0} ; u_{1}, \cdots, u_{s}\right)\left[K_{0}(M): K_{0}\left(y^{\prime}\right)\right]$. On the other hand, the left side is equal to $[Z: K(y)((u))]$, that is, to $e\left(\mathfrak{N}_{U}(M) ; u_{1}, \cdots, u_{s}\right)\left[K(M): K\left(y^{\prime}\right)\right]$. It follows that $e\left(\mathfrak{N}_{U}(M) ; u_{1}, \cdots, u_{s}\right)$ is an integral multiple of the fraction $\left[K_{0}(M): K_{0}\left(y^{\prime}\right)\right] /\left[K(M): K\left(y^{\prime}\right)\right]$. Let $L$ be the field generated by $K_{0}$ and

(41) Set $Y=K_{0}(y), Y^{*}=K_{0}^{*}(y)$. Multiplying $\xi_{1}, \cdots, \xi_{h}$ by some element of $Y[[u]]$, we obtain elements $\xi_{1}^{\prime}, \cdots, \xi_{h}^{\prime}$ which are in $K(y)((u))$ and which are integral over $Y[[u]]$. Since $K(y)[[u]]$ is integrally closed, these elements are power series in the quantities $u$ with coefficients in $K(y)$. Making use of Lemma $1, \S 2$ in my paper Some properties of ideals in rings of power series, Trans. Amer. Math. Soc. vol. 55 (1944) p. 72, we see that the coefficients of these power series are algebraic over $Y$. Since $Y^{*}$ is algebraically closed in $K(y)$ (cf. Proposition 6a in my paper quoted above), the coefficients of $\xi_{1}^{\prime}, \ldots, \xi_{h}^{\prime}$ are in $Y^{*}$. It will therefore be sufficient to prove that, if an element $\eta \in Y^{*}[[u]]$ is algebraic and separable over $Y((u))$, then $\eta \in Y((u))$. By assumption, $\eta$ satisfies an equation $F(\eta)=0$, where $F$ is a polynomial with coefficients in $Y((u))$ and $F^{\prime}(\eta) \neq 0$. Let $\mathfrak{u}$ be the maximal prime ideal in $Y^{*}[[u]]$, and let $h$ be an exponent such that $F^{\prime}(\eta) \notin \mathfrak{u}^{h+1}$. Write $\eta=\sum_{i=0}^{\infty} P_{i}(u)$, where each $P_{i}$ is a form of degree $i$, and let $k$ be an index greater than $h$. Set $\eta_{k}=\sum_{i=0}^{k-1} P_{i}(u), R_{k}=\eta-\eta_{k}$; using Taylor's formula, we obtain $F\left(\eta_{k}\right)+R_{k} F^{\prime}\left(\eta_{k}\right) \equiv 0\left(\bmod \mathfrak{u}^{2 k}\right)$. Because $2 k>k+h$, we see immediately that the coefficients of $P_{k}$ are in the field obtained by adjunction to $Y$ of the coefficients of the polynomial $\eta_{k}$. Thus, all coefficients of the power series $\eta$ are in the field obtained by adjunction to $Y$ of the coefficients of $\eta_{h}$, from which it follows that $\eta^{p^{f}} \in Y((u))$ for some $f>0$, whence $\eta \in Y((u))$ because $\eta$ is separable over $Y((u))$. 
the field of definition of $M$. Then $L / K_{0}$ is a finite purely inseparable algebraic extension and $\left[K(M): K\left(y^{\prime}\right)\right]=\left[L(M): L\left(y^{\prime}\right)\right]$. On the other hand, it follows immediately from the proof of Proposition 1 and from Proposition 3 that $\left[K_{0}(M): K_{0} \cdot\left(y^{\prime}\right)\right] /\left[L(M): L\left(y^{\prime}\right)\right]$ is equal to the level of inseparability of the extension $K_{0}(M) / K_{0}$. Proposition 4 is thereby proved.

THeOREM 9. Let $\Omega$ be a variety in $A^{n}(X)$ and let $U$ and $V$ be two subvarieties of $\Omega$. Let $K_{0}$ be a subfield of $K$ in which $U, V$ and $\Omega$ are definable. Then every component of the intersection of $U$ and $V$ is definable over a field which is algebraic over $K_{0}$. If $M$ is a proper component of the intersection of $U$ and $V$ with respect to $\Omega$, then $i_{\Omega}(M ; U \cdot V)$ is divisible by the level of inseparability of the extension $K_{0}(M) / K_{0}$.

Let $A^{n}\left(X^{\prime}\right)$ be a copy of the space $A^{n}(X)$ and let $V^{\prime}$ be the copy of $V$ in $A^{n}\left(X^{\prime}\right)$. If $\Delta$ is the diagonal of $A^{n}(X) \times A^{n}\left(X^{\prime}\right)$, and if $M$ is a component of the intersection of $U$ and $V$, then $M^{\Delta}$ is a component of the intersection of $U \times V^{\prime}$ and $\Delta$. Let $x_{i}$ and $x_{i}^{\prime}$ be the functions induced on $U \times V^{\prime}$ by $X_{i}$ and $X_{i}^{\prime}$ respectively. Then the $n$ functions $x_{i}^{\prime}-x_{i}$ generate in $\mathfrak{R}_{U \times V^{\prime}}\left(M^{\Delta}\right)$ an ideal which is primary for the ideal of nonunits; on the other hand, these functions belong to $K_{0}\left[U \times V^{\prime}\right]$. The first part of Theorem 9 is then proved exactly in th e same way as the first part of Proposition 4.

If $\omega=\operatorname{dim} \Omega$, we can find $\omega$ indices $i_{1}, \cdots, i_{\omega}$ such that $i_{\Omega}(M ; U \cdot V)$ $=e\left(\mathfrak{R}_{U \times V^{\prime}}\left(M^{\Delta}\right) ; x_{i_{1}}^{\prime}-x_{i_{1}}, \cdots, x_{i_{\omega}}^{\prime}-x_{i_{\omega}}\right)$ (cf. Definition 3, $\$ 4$, p. 67). The second part of Theorem 9 follows therefore immediately from Proposition 4.

Princeton University, Princeton, N. J. 